\title{
The Motion of Point Particles in Curved Spacetime
}

\author{
Eric Poisson \\ Department of Physics \\ University of Guelph \\ Guelph, Ontario \\ Canada N1G 2W1 \\ and \\ Perimeter Institute for Theoretical Physics \\ 35 King Street North \\ Waterloo, Ontario \\ Canada N2J 2W9 \\ email: poisson@physics.uoguelph.ca \\ http://www.physics. uoguelph.ca/poisson/research/ \\ Accepted on 26 March 2004 \\ Published on 27 May 2004 \\ http://www.livingreviews.org/lrr-2004-6 \\ Living Reviews in Relativity \\ Published by the Max Planck Institute for Gravitational Physics \\ Albert Einstein Institute, Germany
}

\begin{abstract}
This review is concerned with the motion of a point scalar charge, a point electric charge, and a point mass in a specified background spacetime. In each of the three cases the particle produces a field that behaves as outgoing radiation in the wave zone, and therefore removes energy from the particle. In the near zone the field acts on the particle and gives rise to a self-force that prevents the particle from moving on a geodesic of the background spacetime. The self-force contains both conservative and dissipative terms, and the latter are responsible for the radiation reaction. The work done by the self-force matches the energy radiated away by the particle.

The field's action on the particle is difficult to calculate because of its singular nature: The field diverges at the position of the particle. But it is possible to isolate the field's singular part and show that it exerts no force on the particle - its only effect is to contribute to the particle's inertia. What remains after subtraction is a smooth field that is fully responsible for the self-force. Because this field satisfies a homogeneous wave equation, it can be thought of as a free (radiative) field that interacts with the particle; it is this interaction that gives rise to the self-force.

The mathematical tools required to derive the equations of motion of a point scalar charge, a point electric charge, and a point mass in a specified background spacetime are developed here from scratch. The review begins with a discussion of the basic theory of bitensors (Section 2). It then applies the theory to the construction of convenient coordinate systems to chart a neighbourhood of the particle's word line (Section 3). It continues with a thorough discussion of Green's functions in curved spacetime (Section 4). The review concludes with a detailed derivation of each of the three equations of motion (Section 5).
\end{abstract}

(C) Max Planck Society and the authors.

Further information on copyright is given at http://relativity.livingreviews.org/Info/Copyright/

For permission to reproduce the article please contact livrev@aei.mpg.de. 


\section{Article Amendments}

On author request a Living Reviews article can be amended to include errata and small additions to ensure that the most accurate and up-to-date information possible is provided. For detailed documentation of amendments, please go to the article's online version at

$$
\text { http://www . livingreviews .org//rr-2004-6 }
$$

Owing to the fact that a Living Reviews article can evolve over time, we recommend to cite the article as follows:

Eric Poisson,

"The Motion of Point Particles in Curved Spacetime",

Living Rev. Relativity, 7, (2004), 6. [Online Article]: cited [<date>], http://www.livingreviews.org/lrr-2004-6

The date in 'cited $[<$ date $>]$ ' then uniquely identifies the version of the article you are referring to. 


\section{Contents}

1 Introduction and Summary $\quad 7$

1.1 Invitation . . . . . . . . . . . . . . . . . . . . . . . 7

1.2 Radiation reaction in flat spacetime . . . . . . . . . . . . . . . . . . . 7

1.3 Green's functions in flat spacetime . . . . . . . . . . . . . . . . . . . . . . . . 9

1.4 Green's functions in curved spacetime . . . . . . . . . . . . . . . . . . 10

1.5 World line and retarded coordinates . . . . . . . . . . . . . . . . 13

1.6 Retarded, singular, and radiative electromagnetic fields of a point electric charge . 15

1.7 Motion of an electric charge in curved spacetime . . . . . . . . . . . . . . 16

1.8 Motion of a scalar charge in curved spacetime . . . . . . . . . . . . . . . . 17

1.9 Motion of a point mass, or a black hole, in a background spacetime . . . . . . . . 18

1.10 Evaluation of the self-force . . . . . . . . . . . . . . . . . 20

1.11 Organization of this review . . . . . . . . . . . . . . . . . . . 22

2 General Theory of Bitensors $\quad 24$

2.1 Synge's world function . . . . . . . . . . . . . . . . . . . . . . . 24

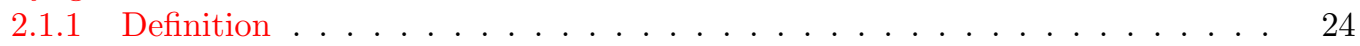

2.1.2 Differentiation of the world function . . . . . . . . . . . . . . . . . . . . 24

2.1.3 Evaluation of first derivatives . . . . . . . . . . . . . . . . . . . . . . . . . . .

2.1.4 Congruence of geodesics emanating from $x^{\prime} \ldots \ldots \ldots$

2.2 Coincidence limits . . . . . . . . . . . . . . . . . . . . . . . . . . . . . . . . . . . . . . . . .

2.2.1 Computation of coincidence limits . . . . . . . . . . . . . . . . . . 27

2.2.2 Derivation of Synge's rule . . . . . . . . . . . . . . . . . . . . . . . . . . . . . . . . . . . . . . . . . . . . .

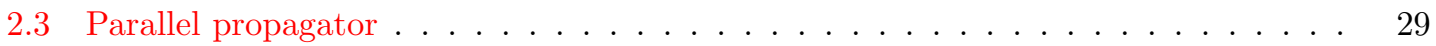

2.3.1 Tetrad on $\beta \ldots \ldots \ldots \ldots \ldots \ldots$. . . . . . . . . . . . . . . . . . . . . . . . . . . . .

2.3.2 Definition and properties of the parallel propagator . . . . . . . . . . 29

2.3 .3 Coincidence limits . . . . . . . . . . . . . . . . . . . . . . . . . . . . .

2.4 Expansion of bitensors near coincidence . . . . . . . . . . . . . . . . . . . . . . . . . . . . .

2.4 General method . . . . . . . . . . . . . . . . . . . . . . . . . . . . . . . . .

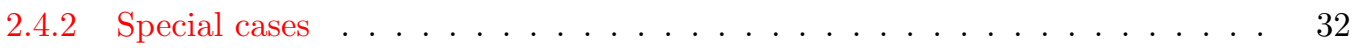

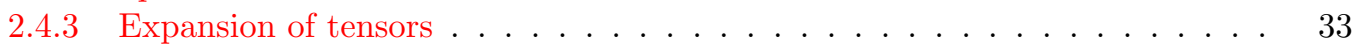

2.5 Van Vleck determinant . . . . . . . . . . . . . . . . . . . . . . . . . . . . . . . . . . . . . . . . . .

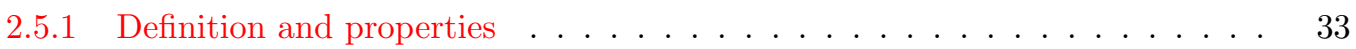

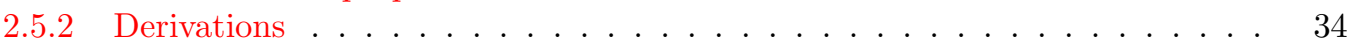

3 Coordinate Systems $\quad 36$

3.1 Riemann normal coordinates . . . . . . . . . . . . . . . . . 36

3.1 Definition and coordinate transformation . . . . . . . . . . . . 36

3.1 .2 Metric near $x^{\prime} \ldots \ldots \ldots \ldots$

3.2 Fermi normal coordinates . . . . . . . . . . . . . . . . . . 37

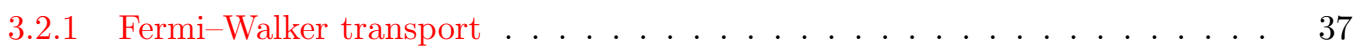

3.2.2 Tetrad and dual tetrad on $\gamma \ldots \ldots . \ldots . \ldots . \ldots . \ldots . \ldots 38$

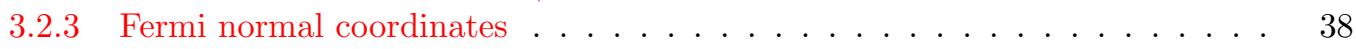

3.2.4 Coordinate displacements near $\gamma \ldots \ldots . \ldots . \ldots . \ldots . \ldots$

3.2 .5 Metric near $\gamma \ldots \ldots \ldots$. . . . . . . . . . . . . . . . . . . . . . . . . 40

3.2.6 Thorne-Hartle coordinates . . . . . . . . . . . . . . 40

3.3 Retarded coordinates . . . . . . . . . . . . . . . . . . . . . . . 42

3.3.1 Geometrical elements . . . . . . . . . . . . . . . . . . . . 42

3.3.2 Definition of the retarded coordinates . . . . . . . . . . . . . 42 
3.3.3 The scalar field $r(x)$ and the vector field $k^{\alpha}(x) \ldots \ldots . \ldots 43$

3.3.4 Frame components of tensor fields on the world line . . . . . . . . . . . . 44

3.3.5 Coordinate displacements near $\gamma \ldots \ldots$. . . . . . . . . . . . . . . . . . . 46

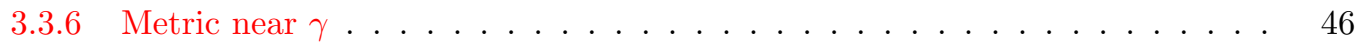

3.3.7 Transformation to angular coordinates . . . . . . . . . . . . . . . . . . . . 47

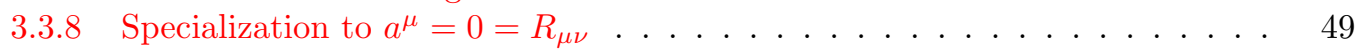

3.4 Transformation between Fermi and retarded coordinates; advanced point . . . . . . 50

3.4.1 From retarded to Fermi coordinates . . . . . . . . . . . . . . 51

3.4.2 From Fermi to retarded coordinates . . . . . . . . . . . . . . 53

3.4.3 Transformation of the tetrads at $x \ldots \ldots \ldots \ldots$. . . . . . . . . . . . . . . . . . . . 54

3.4 .4 Advanced point . . . . . . . . . . . . . . . 56

4 Green's Functions $\quad \mathbf{5 8}$

4.1 Scalar Green's functions in flat spacetime . . . . . . . . . . . . . . . . . . 58

4.1.1 Green's equation for a massive scalar field . . . . . . . . . . . . . . 58

4.1 .2 Integration over the source . . . . . . . . . . . . . . . . . . . . . . . . . . . . . . . . . . . 58

4.1 .3 Singular part of $g(\sigma) \ldots \ldots \ldots \ldots \ldots$. . . . . . . . . . . . . . . . . . . . . . . . . . . . . . . 59

4.1 .4 Smooth part of $g(\sigma) \ldots \ldots \ldots \ldots$. . . . . . . . . . . . . . . . . . . . . . . 60

4.1.5 Advanced distributional methods . . . . . . . . . . . . . . . 60

4.1.6 Alternative computation of the Green's functions . . . . . . . . . . . . . 62

4.2 Distributions in curved spacetime . . . . . . . . . . . . . . . . 63

4.2 .1 Invariant Dirac distribution . . . . . . . . . . . . . . . . . . 63

4.2 Light-cone distributions . . . . . . . . . . . . . . . . . . . . . 64

4.3 Scalar Green's functions in curved spacetime . . . . . . . . . . . . . . . 65

4.3.1 Green's equation for a massless scalar field in curved spacetime . . . . . . 65

4.3.2 Hadamard construction of the Green's functions . . . . . . . . . . . . . 65

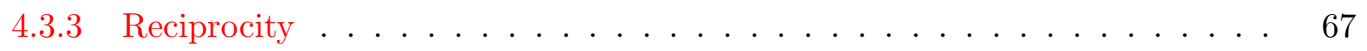

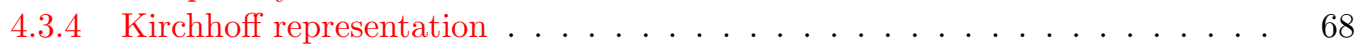

4.3.5 Singular and radiative Green's functions . . . . . . . . . . . . . . . . 69

4.3.6 Example: Cosmological Green's functions . . . . . . . . . . . . . . . . . . . . 72

4.4 Electromagnetic Green's functions . . . . . . . . . . . . . . . . . . . . . . . 74

4.4.1 Equations of electromagnetism . . . . . . . . . . . . . . . . . 74

4.4.2 Hadamard construction of the Green's functions . . . . . . . . . . . . . . 74

4.4.3 Reciprocity and Kirchhoff representation . . . . . . . . . . . . . . . 76

4.4.4 Singular and radiative Green's functions . . . . . . . . . . . . . . . 76

4.5 Gravitational Green's functions . . . . . . . . . . . . . . . . . . . . 78

4.5.1 Equations of linearized gravity . . . . . . . . . . . . . . 78

4.5.2 Hadamard construction of the Green's functions . . . . . . . . . . . . . 79

4.5.3 Reciprocity and Kirchhoff representation . . . . . . . . . . . . . . . . 81

4.5.4 Singular and radiative Green's functions . . . . . . . . . . . . . . 81

5 Motion of Point Particles $\quad 83$

5.1 Motion of a scalar charge . . . . . . . . . . . . . . . . . . 83

5.1 Dynamics of a point scalar charge . . . . . . . . . . . . . . . 83

5.1 .2 Retarded potential near the world line . . . . . . . . . . . . . . . 84

5.1.3 Field of a scalar charge in retarded coordinates . . . . . . . . . . . . . . 85

5.1.4 Field of a scalar charge in Fermi normal coordinates . . . . . . . . . . . . 86

5.1 .5 Singular and radiative fields . . . . . . . . . . . . . . . . . . . 88

5.1 .6 Equations of motion . . . . . . . . . . . . . . . . . . . . . . . 91

5.2 Motion of an electric charge . . . . . . . . . . . . . . . . . . . . 93 
5.2.1 Dynamics of a point electric charge . . . . . . . . . . . . . . 93

5.2.2 Retarded potential near the world line . . . . . . . . . . . . . . . . . . 94

5.2 .3 Electromagnetic field in retarded coordinates . . . . . . . . . . . . . . 95

5.2.4 Electromagnetic field in Fermi normal coordinates . . . . . . . . . . . . . . 96

5.2 .5 Singular and radiative fields . . . . . . . . . . . . . . . . . . . . . . . . . . . . . . . . . . . . . . . .

5.2 .6 Equations of motion . . . . . . . . . . . . . . . . . . 100

5.3 Motion of a point mass . . . . . . . . . . . . . . . . . . . . . 102

5.3 .1 Dynamics of a point mass . . . . . . . . . . . . . . . . . . . . . . . . . . . . . . . . . . .

5.3.2 Retarded potentials near the world line . . . . . . . . . . . . . . 105

5.3.3 Gravitational field in retarded coordinates . . . . . . . . . . . . . . . 106

5.3.4 Gravitational field in Fermi normal coordinates . . . . . . . . . . . . . . 107

5.3.5 Singular and radiative fields . . . . . . . . . . . . . . . . . . . . . . . . . . . . . . . . . . . . . . .

5.3 .6 Equations of motion . . . . . . . . . . . . . . . . . . 110

5.3.7 Gauge dependence of the equations of motion . . . . . . . . . . . . . . 111

5.4 Motion of a small black hole . . . . . . . . . . . . . . . . . . . . . . . . . . . 112

5.4.1 Matched asymptotic expansions . . . . . . . . . . . . . . . 112

5.4 .2 Metric in the internal zone . . . . . . . . . . . . . . . 115

5.4 .3 Metric in the external zone . . . . . . . . . . . . . . . . 118

5.4.4 Transformation from external to internal coordinates . . . . . . . . . . . . 121

5.4.5 Motion of the black hole in the background spacetime . . . . . . . . . . . 123

5.5 Concluding remarks . . . . . . . . . . . . . . . . . . . . 124

5.5.1 Conservation of energy-momentum . . . . . . . . . . . . . . 124

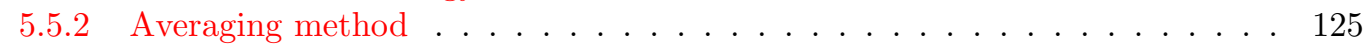

5.5.3 Detweiler-Whiting axiom . . . . . . . . . . . . . . . 126

5.5.4 Matched asymptotic expansions . . . . . . . . . . . . . . . 127

5.5.5 Evaluation of the gravitational self-force . . . . . . . . . . . . . . . . . 127

5.5.6 Beyond the self-force . . . . . . . . . . . . . . . . . . 129

6 Acknowledgments $\quad 130$

$\begin{array}{lr}\text { References } & 131\end{array}$ 



\section{Introduction and Summary}

\subsection{Invitation}

The motion of a point electric charge in flat spacetime was the subject of active investigation since the early work of Lorentz, Abrahams, and Poincaré, until Dirac [25] produced a proper relativistic derivation of the equations of motion in 1938. (The field's early history is well related in [52].) In 1960 DeWitt and Brehme [24] generalized Dirac's result to curved spacetimes, and their calculation was corrected by Hobbs [29] several years later. In 1997 the motion of a point mass in a curved background spacetime was investigated by Mino, Sasaki, and Tanaka [39], who derived an expression for the particle's acceleration (which is not zero unless the particle is a test mass); the same equations of motion were later obtained by Quinn and Wald [49] using an axiomatic approach. The case of a point scalar charge was finally considered by Quinn in 2000 [48], and this led to the realization that the mass of a scalar particle is not necessarily a constant of the motion.

This article reviews the achievements described in the preceding paragraph; it is concerned with the motion of a point scalar charge $q$, a point electric charge $e$, and a point mass $m$ in a specified background spacetime with metric $g_{\alpha \beta}$. These particles carry with them fields that behave as outgoing radiation in the wave zone. The radiation removes energy and angular momentum from the particle, which then undergoes a radiation reaction - its world line cannot be simply a geodesic of the background spacetime. The particle's motion is affected by the near-zone field which acts directly on the particle and produces a self-force. In curved spacetime the self-force contains a radiation-reaction component that is directly associated with dissipative effects, but it contains also a conservative component that is not associated with energy or angular-momentum transport. The self-force is proportional to $q^{2}$ in the case of a scalar charge, proportional to $e^{2}$ in the case of an electric charge, and proportional to $m^{2}$ in the case of a point mass.

In this review I derive the equations that govern the motion of a point particle in a curved background spacetime. The presentation is entirely self-contained, and all relevant materials are developed $a b$ initio. The reader, however, is assumed to have a solid grasp of differential geometry and a deep understanding of general relativity. The reader is also assumed to have unlimited stamina, for the road to the equations of motion is a long one. One must first assimilate the basic theory of bitensors (Section 2), then apply the theory to construct convenient coordinate systems to chart a neighbourhood of the particle's world line (Section 3). One must next formulate a theory of Green's functions in curved spacetimes (Section 4), and finally calculate the scalar, electromagnetic, and gravitational fields near the world line and figure out how they should act on the particle (Section 5). The review is very long, but the payoff, I hope, will be commensurate.

In this introductory section I set the stage and present an impressionistic survey of what the review contains. This should help the reader get oriented and acquainted with some of the ideas and some of the notation. Enjoy!

\subsection{Radiation reaction in flat spacetime}

Let us first consider the relatively simple and well-understood case of a point electric charge $e$ moving in flat spacetime $[52,30,56,47]$. The charge produces an electromagnetic vector potential $A^{\alpha}$ that satisfies the wave equation

$$
\square A^{\alpha}=-4 \pi j^{\alpha}
$$

together with the Lorenz gauge condition $\partial_{\alpha} A^{\alpha}=0$. (On page 294 in [30] Jackson explains why the term "Lorenz gauge" is preferable to "Lorentz gauge".) The vector $j^{\alpha}$ is the charge's current density, which is formally written in terms of a four-dimensional Dirac functional supported on the charge's world line: The density is zero everywhere, except at the particle's position where it is infinite. For concreteness we will imagine that the particle moves around a centre (perhaps 
another charge, which is taken to be fixed) and that it emits outgoing radiation. We expect that the charge will undergo a radiation reaction and that it will spiral down toward the centre. This effect must be accounted for by the equations of motion, and these must therefore include the action of the charge's own field, which is the only available agent that could be responsible for the radiation reaction. We seek to determine this self-force acting on the particle.

An immediate difficulty presents itself: The vector potential, and also the electromagnetic field tensor, diverge on the particle's world line, because the field of a point charge is necessarily infinite at the charge's position. This behaviour makes it most difficult to decide how the field is supposed to act on the particle.

Difficult but not impossible. To find a way around this problem I note first that the situation considered here, in which the radiation is propagating outward and the charge is spiraling inward, breaks the time-reversal invariance of Maxwell's theory. A specific time direction was adopted when, among all possible solutions to the wave equation, we chose $A_{\text {ret }}^{\alpha}$, the retarded solution, as the physically-relevant solution. Choosing instead the advanced solution $A_{\mathrm{adv}}^{\alpha}$ would produce a time-reversed picture in which the radiation is propagating inward and the charge is spiraling outward. Alternatively, choosing the linear superposition

$$
A_{\mathrm{S}}^{\alpha}=\frac{1}{2}\left(A_{\mathrm{ret}}^{\alpha}+A_{\mathrm{adv}}^{\alpha}\right)
$$

would restore time-reversal invariance: Outgoing and incoming radiation would be present in equal amounts, there would be no net loss nor gain of energy by the system, and the charge would not undergo any radiation reaction. In Equation (2) the subscript 'S' stands for 'symmetric', as the vector potential depends symmetrically upon future and past.

My second key observation is that while the potential of Equation (2) does not exert a force on the charged particle, it is just as singular as the retarded potential in the vicinity of the world line. This follows from the fact that $A_{\text {ret }}^{\alpha}, A_{\text {adv }}^{\alpha}$, and $A_{\mathrm{S}}^{\alpha}$ all satisfy Equation (1), whose source term is infinite on the world line. So while the wave-zone behaviours of these solutions are very different (with the retarded solution describing outgoing waves, the advanced solution describing incoming waves, and the symmetric solution describing standing waves), the three vector potentials share the same singular behaviour near the world line - all three electromagnetic fields are dominated by the particle's Coulomb field and the different asymptotic conditions make no difference close to the particle. This observation gives us an alternative interpretation for the subscript 'S': It stands for 'singular' as well as 'symmetric'.

Because $A_{\mathrm{S}}^{\alpha}$ is just as singular as $A_{\text {ret }}^{\alpha}$, removing it from the retarded solution gives rise to a potential that is well behaved in a neighbourhood of the world line. And because $A_{\mathrm{S}}^{\alpha}$ is known not to affect the motion of the charged particle, this new potential must be entirely responsible for the radiation reaction. We therefore introduce the new potential

$$
A_{\mathrm{R}}^{\alpha}=A_{\mathrm{ret}}^{\alpha}-A_{\mathrm{S}}^{\alpha}=\frac{1}{2}\left(A_{\mathrm{ret}}^{\alpha}-A_{\mathrm{adv}}^{\alpha}\right)
$$

and postulate that it, and it alone, exerts a force on the particle. The subscript ' $\mathrm{R}$ ' stands for 'regular', because $A_{\mathrm{R}}^{\alpha}$ is nonsingular on the world line. This property can be directly inferred from the fact that the regular potential satisfies the homogeneous version of Equation (1), $\square A_{\mathrm{R}}^{\alpha}=0$; there is no singular source to produce a singular behaviour on the world line. Since $A_{\mathrm{R}}^{\alpha}$ satisfies the homogeneous wave equation, it can be thought of as a free radiation field, and the subscript ' $R$ ' could also stand for 'radiative'.

The self-action of the charge's own field is now clarified: A singular potential $A_{\mathrm{S}}^{\alpha}$ can be removed from the retarded potential and shown not to affect the motion of the particle. (Establishing this last statement requires a careful analysis that is presented in the bulk of the paper; what really happens is that the singular field contributes to the particle's inertia and renormalizes its mass.)

Living Reviews in Relativity

http: //www . livingreviews . org/lrr-2004-6 
What remains is a well-behaved potential $A_{\mathrm{R}}^{\alpha}$ that must be solely responsible for the radiation reaction. From the radiative potential we form an electromagnetic field tensor $F_{\alpha \beta}^{\mathrm{R}}=\partial_{\alpha} A_{\beta}^{\mathrm{R}}-\partial_{\beta} A_{\alpha}^{\mathrm{R}}$, and we take the particle's equations of motion to be

$$
m a_{\mu}=f_{\mu}^{\mathrm{ext}}+e F_{\mu \nu}^{\mathrm{R}} u^{\nu},
$$

where $u^{\mu}=d z^{\mu} / d \tau$ is the charge's four-velocity $\left(z^{\mu}(\tau)\right.$ gives the description of the world line and $\tau$ is proper time), $a^{\mu}=d u^{\mu} / d \tau$ its acceleration, $m$ its (renormalized) mass, and $f_{\text {ext }}^{\mu}$ an external force also acting on the particle. Calculation of the radiative field yields the more concrete expression

$$
m a^{\mu}=f_{\mathrm{ext}}^{\mu}+\frac{2 e^{2}}{3 m}\left(\delta^{\mu}{ }_{\nu}+u^{\mu} u_{\nu}\right) \frac{d f_{\mathrm{ext}}^{\nu}}{d \tau},
$$

in which the second-term is the self-force that is responsible for the radiation reaction. We observe that the self-force is proportional to $e^{2}$, it is orthogonal to the four-velocity, and it depends on the rate of change of the external force. This is the result that was first derived by Dirac [25] ${ }^{1}$.

\subsection{Green's functions in flat spacetime}

To see how Equation (5) can eventually be generalized to curved spacetimes, I introduce a new layer of mathematical formalism and show that the decomposition of the retarded potential into symmetric-singular and regular-radiative pieces can be performed at the level of the Green's functions associated with Equation (1). The retarded solution to the wave equation can be expressed as

$$
A_{\mathrm{ret}}^{\alpha}(x)=\int G_{+\beta^{\prime}}^{\alpha}\left(x, x^{\prime}\right) j^{\beta^{\prime}}\left(x^{\prime}\right) d V^{\prime}
$$

in terms of the retarded Green's function $G_{+\beta^{\prime}}^{\alpha}\left(x, x^{\prime}\right)=\delta_{\beta^{\prime}}^{\alpha} \delta\left(t-t^{\prime}-\left|\boldsymbol{x}-\boldsymbol{x}^{\prime}\right|\right) /\left|\boldsymbol{x}-\boldsymbol{x}^{\prime}\right|$. Here $x=(t, \boldsymbol{x})$ is an arbitrary field point, $x^{\prime}=\left(t^{\prime}, \boldsymbol{x}^{\prime}\right)$ is a source point, and $d V^{\prime} \equiv d^{4} x^{\prime}$; tensors at $x$ are identified with unprimed indices, while primed indices refer to tensors at $x^{\prime}$. Similarly, the advanced solution can be expressed as

$$
A_{\mathrm{adv}}^{\alpha}(x)=\int G_{-\beta^{\prime}}^{\alpha}\left(x, x^{\prime}\right) j^{\beta^{\prime}}\left(x^{\prime}\right) d V^{\prime}
$$

in terms of the advanced Green's function $G_{-\beta^{\prime}}^{\alpha}\left(x, x^{\prime}\right)=\delta_{\beta^{\prime}}^{\alpha} \delta\left(t-t^{\prime}+\left|\boldsymbol{x}-\boldsymbol{x}^{\prime}\right|\right) /\left|\boldsymbol{x}-\boldsymbol{x}^{\prime}\right|$. The retarded Green's function is zero whenever $x$ lies outside of the future light cone of $x^{\prime}$, and $G_{+\beta^{\prime}}^{\alpha}\left(x, x^{\prime}\right)$ is infinite at these points. On the other hand, the advanced Green's function is zero whenever $x$ lies outside of the past light cone of $x^{\prime}$, and $G_{-\beta^{\prime}}^{\alpha}\left(x, x^{\prime}\right)$ is infinite at these points. The retarded and advanced Green's functions satisfy the reciprocity relation

$$
G_{\beta^{\prime} \alpha}^{-}\left(x^{\prime}, x\right)=G_{\alpha \beta^{\prime}}^{+}\left(x, x^{\prime}\right) ;
$$

this states that the retarded Green's function becomes the advanced Green's function (and vice versa) when $x$ and $x^{\prime}$ are interchanged. by

From the retarded and advanced Green's functions we can define a singular Green's function

$$
G_{\mathrm{S} \beta^{\prime}}^{\alpha}\left(x, x^{\prime}\right)=\frac{1}{2}\left[G_{+\beta^{\prime}}^{\alpha}\left(x, x^{\prime}\right)+G_{-\beta^{\prime}}^{\alpha}\left(x, x^{\prime}\right)\right]
$$

\footnotetext{
${ }^{1}$ Dirac's original expression actually involved the rate of change of the acceleration vector on the right-hand side. The resulting equation gives rise to the well-known problem of runaway solutions. To avoid such unphysical behaviour I have submitted Dirac's equation to a reduction-of-order procedure whereby $d a^{\nu} / d \tau$ is replaced with $m^{-1} d f_{\text {ext }}^{\nu} / d \tau$. This procedure is explained and justified, for example, in [47, 26].
} 
and a radiative Green's function by

$$
G_{\mathrm{R} \beta^{\prime}}^{\alpha}\left(x, x^{\prime}\right)=G_{+\beta^{\prime}}^{\alpha}\left(x, x^{\prime}\right)-G_{\mathrm{S} \beta^{\prime}}^{\alpha}\left(x, x^{\prime}\right)=\frac{1}{2}\left[G_{+\beta^{\prime}}^{\alpha}\left(x, x^{\prime}\right)-G_{-\beta^{\prime}}^{\alpha}\left(x, x^{\prime}\right)\right] .
$$

By virtue of Equation (8) the singular Green's function is symmetric in its indices and arguments: $G_{\beta^{\prime} \alpha}^{\mathrm{S}}\left(x^{\prime}, x\right)=G_{\alpha \beta^{\prime}}^{\mathrm{S}}\left(x, x^{\prime}\right)$. The radiative Green's function, on the other hand, is antisymmetric. The potential

$$
A_{\mathrm{S}}^{\alpha}(x)=\int G_{\mathrm{S} \beta^{\prime}}^{\alpha}\left(x, x^{\prime}\right) j^{\beta^{\prime}}\left(x^{\prime}\right) d V^{\prime}
$$

satisfies the wave equation of Equation (1) and is singular on the world line, while

$$
A_{\mathrm{R}}^{\alpha}(x)=\int G_{\mathrm{R} \beta^{\prime}}^{\alpha}\left(x, x^{\prime}\right) j^{\beta^{\prime}}\left(x^{\prime}\right) d V^{\prime}
$$

satisfies the homogeneous equation $\square A^{\alpha}=0$ and is well behaved on the world line.

Equation (6) implies that the retarded potential at $x$ is generated by a single event in spacetime: the intersection of the world line and the past light cone of $x$ ' (see Figure 1). I shall call this the retarded point associated with $x$ and denote it $z(u) ; u$ is the retarded time, the value of the propertime parameter at the retarded point. Similarly we find that the advanced potential of Equation (7) is generated by the intersection of the world line and the future light cone of the field point $x$. I shall call this the advanced point associated with $x$ and denote it $z(v) ; v$ is the advanced time, the value of the proper-time parameter at the advanced point.

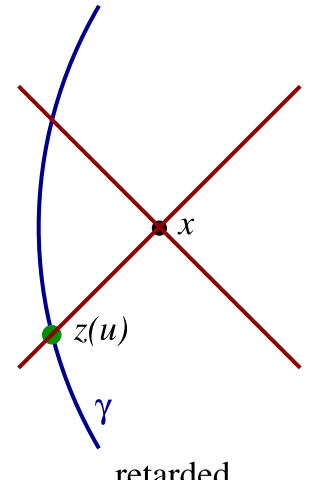

retarded

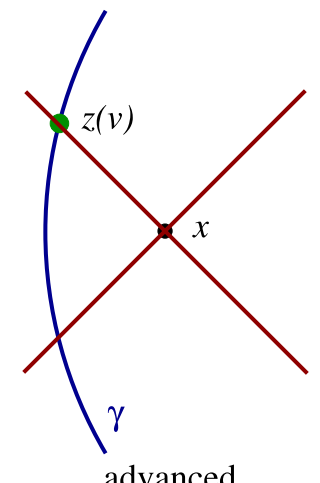

advanced

Figure 1: In flat spacetime, the retarded potential at $x$ depends on the particle's state of motion at the retarded point $z(u)$ on the world line; the advanced potential depends on the state of motion at the advanced point $z(v)$.

\subsection{Green's functions in curved spacetime}

In a curved spacetime with metric $g_{\alpha \beta}$ the wave equation for the vector potential becomes

$$
\square A^{\alpha}-R_{\beta}^{\alpha} A^{\beta}=-4 \pi j^{\alpha},
$$

where $\square=g^{\alpha \beta} \nabla_{\alpha} \nabla_{\beta}$ is the covariant wave operator and $R_{\alpha \beta}$ is the spacetime's Ricci tensor; the Lorenz gauge conditions becomes $\nabla_{\alpha} A^{\alpha}=0$, and $\nabla_{\alpha}$ denotes covariant differentiation. Retarded and advanced Green's functions can be defined for this equation, and solutions to Equation (13) take the same form as in Equations (6) and (7), except that $d V^{\prime}$ now stands for $\sqrt{-g\left(x^{\prime}\right)} d^{4} x^{\prime}$.

Living Reviews in Relativity

http: //www. livingreviews. org/lrr-2004-6 
The causal structure of the Green's functions is richer in curved spacetime: While in flat spacetime the retarded Green's function has support only on the future light cone of $x^{\prime}$, in curved spacetime its support extends inside the light cone as well; $G_{+\beta^{\prime}}^{\alpha}\left(x, x^{\prime}\right)$ is therefore nonzero when $x \in I^{+}\left(x^{\prime}\right)$, which denotes the chronological future of $x^{\prime}$. This property reflects the fact that in curved spacetime, electromagnetic waves propagate not just at the speed of light, but at all speeds smaller than or equal to the speed of light; the delay is caused by an interaction between the radiation and the spacetime curvature. A direct implication of this property is that the retarded potential at $x$ is now generated by the point charge during its entire history prior to the retarded time $u$ associated with $x$ : The potential depends on the particle's state of motion for all times $\tau \leq u$ (see Figure 2).

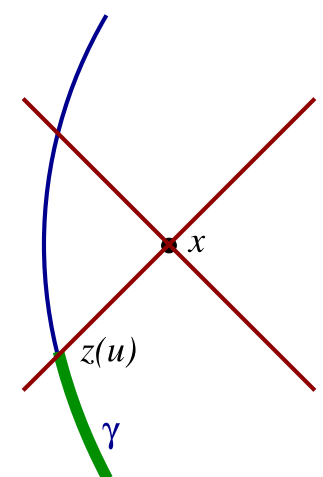

retarded

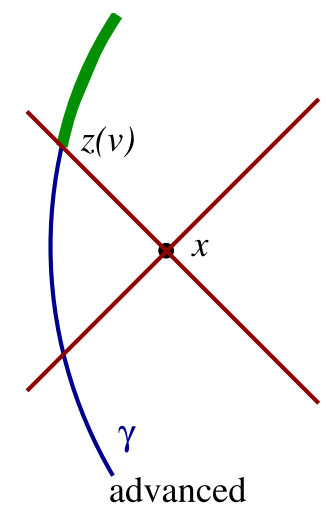

advanced

Figure 2: In curved spacetime, the retarded potential at $x$ depends on the particle's history before the retarded time $u$; the advanced potential depends on the particle's history after the advanced time $v$.

Similar statements can be made about the advanced Green's function and the advanced solution to the wave equation. While in flat spacetime the advanced Green's function has support only on the past light cone of $x^{\prime}$, in curved spacetime its support extends inside the light cone, and $G_{-\beta^{\prime}}^{\alpha}\left(x, x^{\prime}\right)$ is nonzero when $x \in I^{-}\left(x^{\prime}\right)$, which denotes the chronological past of $x^{\prime}$. This implies that the advanced potential at $x$ is generated by the point charge during its entire future history following the advanced time $v$ associated with $x$ : The potential depends on the particle's state of motion for all times $\tau \geq v$.

The physically relevant solution to Equation (13) is obviously the retarded potential $A_{\text {ret }}^{\alpha}(x)$, and as in flat spacetime, this diverges on the world line. The cause of this singular behaviour is still the pointlike nature of the source, and the presence of spacetime curvature does not change the fact that the potential diverges at the position of the particle. Once more this behaviour makes it difficult to figure out how the retarded field is supposed to act on the particle and determine its motion. As in flat spacetime we shall attempt to decompose the retarded solution into a singular part that exerts no force, and a smooth radiative part that produces the entire self-force.

To decompose the retarded Green's function into singular and radiative parts is not a straightforward task in curved spacetime. The flat-spacetime definition for the singular Green's function, Equation (9), cannot be adopted without modification: While the combination half-retarded plus half-advanced Green's functions does have the property of being symmetric, and while the resulting vector potential would be a solution to Equation (13), this candidate for the singular Green's function would produce a self-force with an unacceptable dependence on the particle's future history. For suppose that we made this choice. Then the radiative Green's function would be given by the combination half-retarded minus half-advanced Green's functions, just as in flat spacetime. The 
resulting radiative potential would satisfy the homogeneous wave equation, and it would be smooth on the world line, but it would also depend on the particle's entire history, both past (through the retarded Green's function) and future (through the advanced Green's function). More precisely stated, we would find that the radiative potential at $x$ depends on the particle's state of motion at all times $\tau$ outside the interval $u<\tau<v$; in the limit where $x$ approaches the world line, this interval shrinks to nothing, and we would find that the radiative potential is generated by the complete history of the particle. A self-force constructed from this potential would be highly noncausal, and we are compelled to reject these definitions for the singular and radiative Green's functions.

The proper definitions were identified by Detweiler and Whiting [23], who proposed the following generalization to Equation (9):

$$
G_{\mathrm{S} \beta^{\prime}}^{\alpha}\left(x, x^{\prime}\right)=\frac{1}{2}\left[G_{+\beta^{\prime}}^{\alpha}\left(x, x^{\prime}\right)+G_{-\beta^{\prime}}^{\alpha}\left(x, x^{\prime}\right)-H_{\beta^{\prime}}^{\alpha}\left(x, x^{\prime}\right)\right] .
$$

The two-point function $H_{\beta^{\prime}}^{\alpha}\left(x, x^{\prime}\right)$ is introduced specifically to cure the pathology described in the preceding paragraph. It is symmetric in its indices and arguments, so that $G_{\alpha \beta^{\prime}}^{\mathrm{S}}\left(x, x^{\prime}\right)$ will be also (since the retarded and advanced Green's functions are still linked by a reciprocity relation); and it is a solution to the homogeneous wave equation, $\square H_{\beta^{\prime}}^{\alpha}\left(x, x^{\prime}\right)-R_{\gamma}^{\alpha}(x) H_{\beta^{\prime}}^{\gamma}\left(x, x^{\prime}\right)=0$, so that the singular, retarded, and advanced Green's functions will all satisfy the same wave equation. Furthermore, and this is its key property, the two-point function is defined to agree with the advanced Green's function when $x$ is in the chronological past of $x^{\prime}: H_{\beta^{\prime}}^{\alpha}\left(x, x^{\prime}\right)=G_{-\beta^{\prime}}{ }^{\prime}\left(x, x^{\prime}\right)$ when $x \in I^{-}\left(x^{\prime}\right)$. This ensures that $G_{\mathrm{S} \beta^{\prime}}{ }^{\prime}\left(x, x^{\prime}\right)$ vanishes when $x$ is in the chronological past of $x^{\prime}$. In fact, reciprocity implies that $H_{\beta^{\prime}}^{\alpha}\left(x, x^{\prime}\right)$ will also agree with the retarded Green's function when $x$ is in the chronological future of $x^{\prime}$, and it follows that the symmetric Green's function vanishes also when $x$ is in the chronological future of $x^{\prime}$.

The potential $A_{\mathrm{S}}^{\alpha}(x)$ constructed from the singular Green's function can now be seen to depend on the particle's state of motion at times $\tau$ restricted to the interval $u \leq \tau \leq v$ (see Figure 3). Because this potential satisfies Equation (13), it is just as singular as the retarded potential in the vicinity of the world line. And because the singular Green's function is symmetric in its arguments, the singular potential can be shown to exert no force on the charged particle. (This requires a lengthy analysis that will be presented in the bulk of the paper.)
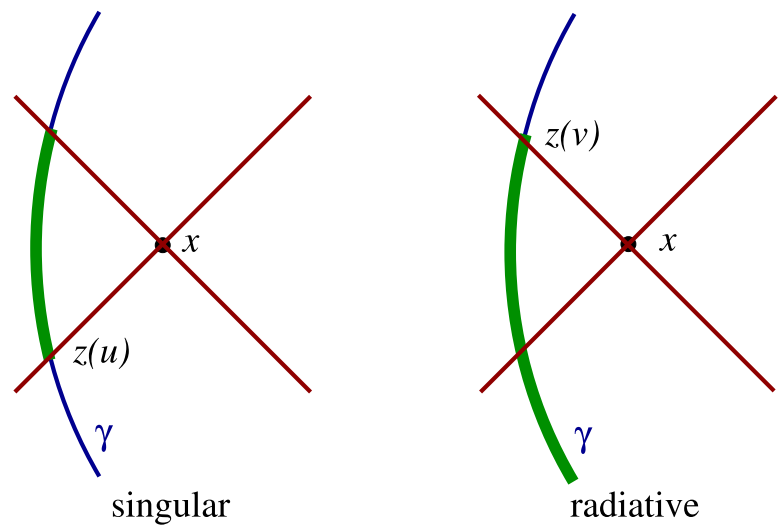

Figure 3: In curved spacetime, the singular potential at $x$ depends on the particle's history during the interval $u \leq \tau \leq v$; for the radiative potential the relevant interval is $-\infty<\tau \leq v$.

Living Reviews in Relativity

http://www. livingreviews.org//rr-2004-6 
The Detweiler-Whiting [23] definition for the radiative Green's function is then

$$
G_{\mathrm{R} \beta^{\prime}}^{\alpha}\left(x, x^{\prime}\right)=G_{+\beta^{\prime}}^{\alpha}\left(x, x^{\prime}\right)-G_{\mathrm{S} \beta^{\prime}}^{\alpha}\left(x, x^{\prime}\right)=\frac{1}{2}\left[G_{+\beta^{\prime}}^{\alpha}\left(x, x^{\prime}\right)-G_{-\beta^{\prime}}^{\alpha}\left(x, x^{\prime}\right)+H_{\beta^{\prime}}^{\alpha}\left(x, x^{\prime}\right)\right] .
$$

The potential $A_{\mathrm{R}}^{\alpha}(x)$ constructed from this depends on the particle's state of motion at all times $\tau$ prior to the advanced time $v: \tau \leq v$. Because this potential satisfies the homogeneous wave equation, it is well behaved on the world line and its action on the point charge is well defined. And because the singular potential $A_{\mathrm{S}}^{\alpha}(x)$ can be shown to exert no force on the particle, we conclude that $A_{\mathrm{R}}^{\alpha}(x)$ alone is responsible for the self-force.

From the radiative potential we form an electromagnetic field tensor $F_{\alpha \beta}^{\mathrm{R}}=\nabla_{\alpha} A_{\beta}^{\mathrm{R}}-\nabla_{\beta} A_{\alpha}^{\mathrm{R}}$, and the curved-spacetime generalization to Equation (4) is

$$
m a_{\mu}=f_{\mu}^{\mathrm{ext}}+e F_{\mu \nu}^{\mathrm{R}} u^{\nu},
$$

where $u^{\mu}=d z^{\mu} / d \tau$ is again the charge's four-velocity, but $a^{\mu}=D u^{\mu} / d \tau$ is now its covariant acceleration.

\subsection{World line and retarded coordinates}

To flesh out the ideas contained in the preceding Section 1.4 I add yet another layer of mathematical formalism and construct a convenient coordinate system to chart a neighbourhood of the particle's world line. In the next Section 1.6 I will display explicit expressions for the retarded, singular, and radiative fields of a point electric charge.

Let $\gamma$ be the world line of a point particle in a curved spacetime. It is described by parametric relations $z^{\mu}(\tau)$ in which $\tau$ is proper time. Its tangent vector is $u^{\mu}=d z^{\mu} / d \tau$ and its acceleration is $a^{\mu}=D u^{\mu} / d \tau$; we shall also encounter $\dot{a}^{\mu} \equiv D a^{\mu} / d \tau$.

On $\gamma$ we erect an orthonormal basis that consists of the four-velocity $u^{\mu}$ and three spatial vectors $e_{a}^{\mu}$ labelled by a frame index $a=(1,2,3)$. These vectors satisfy the relations $g_{\mu \nu} u^{\mu} u^{\nu}=-1$, $g_{\mu \nu} u^{\mu} e_{a}^{\nu}=0$, and $g_{\mu \nu} e_{a}^{\mu} e_{b}^{\nu}=\delta_{a b}$. We take the spatial vectors to be Fermi-Walker transported on the world line: $D e_{a}^{\mu} / d \tau=a_{a} u^{\mu}$, where

$$
a_{a}(\tau)=a_{\mu} e_{a}^{\mu}
$$

are frame components of the acceleration vector; it is easy to show that Fermi-Walker transport preserves the orthonormality of the basis vectors. We shall use the tetrad to decompose various tensors evaluated on the world line. An example was already given in Equation (17) but we shall also encounter frame components of the Riemann tensor,

$$
R_{a 0 b 0}(\tau)=R_{\mu \lambda \nu \rho} e_{a}^{\mu} u^{\lambda} e_{b}^{\nu} u^{\rho}, \quad R_{a 0 b c}(\tau)=R_{\mu \lambda \nu \rho} e_{a}^{\mu} u^{\lambda} e_{b}^{\nu} e_{c}^{\rho}, \quad R_{a b c d}(\tau)=R_{\mu \lambda \nu \rho} e_{a}^{\mu} e_{b}^{\lambda} e_{c}^{\nu} e_{d}^{\rho}
$$

as well as frame components of the Ricci tensor,

$$
R_{00}(\tau)=R_{\mu \nu} u^{\mu} u^{\nu}, \quad R_{a 0}(\tau)=R_{\mu \nu} e_{a}^{\mu} u^{\nu}, \quad R_{a b}(\tau)=R_{\mu \nu} e_{a}^{\mu} e_{b}^{\nu} .
$$

We shall use $\delta_{a b}=\operatorname{diag}(1,1,1)$ and its inverse $\delta^{a b}=\operatorname{diag}(1,1,1)$ to lower and raise frame indices, respectively.

Consider a point $x$ in a neighbourhood of the world line $\gamma$. We assume that $x$ is sufficiently close to the world line that a unique geodesic links $x$ to any neighbouring point $z$ on $\gamma$. The two-point function $\sigma(x, z)$, known as Synge's world function [55], is numerically equal to half the squared geodesic distance between $z$ and $x$; it is positive if $x$ and $z$ are spacelike related, negative if they are timelike related, and $\sigma(x, z)$ is zero if $x$ and $z$ are linked by a null geodesic. We denote its 
gradient $\partial \sigma / \partial z^{\mu}$ by $\sigma_{\mu}(x, z)$, and $-\sigma^{\mu}$ gives a meaningful notion of a separation vector (pointing from $z$ to $x$ ).

To construct a coordinate system in this neighbourhood we locate the unique point $x^{\prime} \equiv z(u)$ on $\gamma$ which is linked to $x$ by a future-directed null geodesic (this geodesic is directed from $x^{\prime}$ to $x$ ); I shall refer to $x^{\prime}$ as the retarded point associated with $x$, and $u$ will be called the retarded time. To tensors at $x^{\prime}$ we assign indices $\alpha^{\prime}, \beta^{\prime}, \ldots$; this will distinguish them from tensors at a generic point $z(\tau)$ on the world line, to which we have assigned indices $\mu, \nu, \ldots$ We have $\sigma\left(x, x^{\prime}\right)=0$, and $-\sigma^{\alpha^{\prime}}\left(x, x^{\prime}\right)$ is a null vector that can be interpreted as the separation between $x^{\prime}$ and $x$.

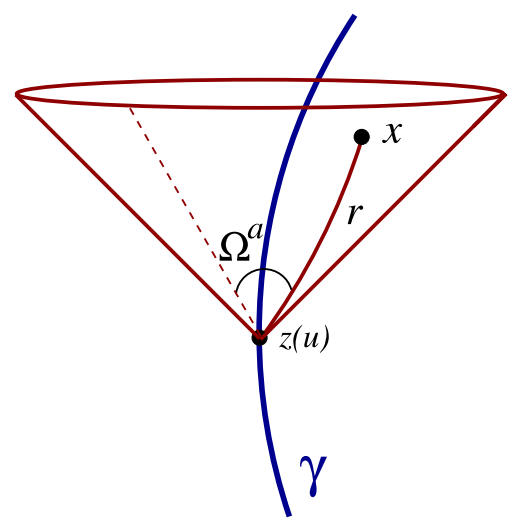

Figure 4: Retarded coordinates of a point $x$ relative to a world line $\gamma$. The retarded time $u$ selects a particular null cone, the unit vector $\Omega^{a} \equiv \hat{x}^{a} / r$ selects a particular generator of this null cone, and the retarded distance $r$ selects a particular point on this generator.

The retarded coordinates of the point $x$ are $\left(u, \hat{x}^{a}\right)$, where $\hat{x}^{a}=-e_{\alpha^{\prime}}^{a} \sigma^{\alpha^{\prime}}$ are the frame components of the separation vector. They come with a straightforward interpretation (see Figure 4). The invariant quantity

$$
r \equiv \sqrt{\delta_{a b} \hat{x}^{a} \hat{x}^{b}}=u_{\alpha^{\prime}} \sigma^{\alpha^{\prime}}
$$

is an affine parameter on the null geodesic that links $x$ to $x^{\prime}$; it can be loosely interpreted as the time delay between $x$ and $x^{\prime}$ as measured by an observer moving with the particle. This therefore gives a meaningful notion of distance between $x$ and the retarded point, and I shall call $r$ the retarded distance between $x$ and the world line. The unit vector

$$
\Omega^{a}=\hat{x}^{a} / r
$$

is constant on the null geodesic that links $x$ to $x^{\prime}$. Because $\Omega^{a}$ is a different constant on each null geodesic that emanates from $x^{\prime}$, keeping $u$ fixed and varying $\Omega^{a}$ produces a congruence of null geodesics that generate the future light cone of the point $x^{\prime}$ (the congruence is hypersurface orthogonal). Each light cone can thus be labelled by its retarded time $u$, each generator on a given light cone can be labelled by its direction vector $\Omega^{a}$, and each point on a given generator can be labelled by its retarded distance $r$. We therefore have a good coordinate system in a neighbourhood of $\gamma$.

To tensors at $x$ we assign indices $\alpha, \beta, \ldots$. These tensors will be decomposed in a tetrad $\left(e_{0}^{\alpha}, e_{a}^{\alpha}\right)$ that is constructed as follows: Given $x$ we locate its associated retarded point $x^{\prime}$ on the world line, as well as the null geodesic that links these two points; we then take the tetrad $\left(u^{\alpha^{\prime}}, e_{a}^{\alpha^{\prime}}\right)$ at $x^{\prime}$ and parallel transport it to $x$ along the null geodesic to obtain $\left(e_{0}^{\alpha}, e_{a}^{\alpha}\right)$.

Living Reviews in Relativity

http: //www . livingreviews . org/lrr-2004-6 


\subsection{Retarded, singular, and radiative electromagnetic fields of a point electric charge}

The retarded solution to Equation (13) is

$$
A^{\alpha}(x)=e \int_{\gamma} G_{+\mu}^{\alpha}(x, z) u^{\mu} d \tau,
$$

where the integration is over the world line of the point electric charge. Because the retarded solution is the physically relevant solution to the wave equation, it will not be necessary to put a label 'ret' on the vector potential.

From the vector potential we form the electromagnetic field tensor $F_{\alpha \beta}$, which we decompose in the tetrad $\left(e_{0}^{\alpha}, e_{a}^{\alpha}\right)$ introduced at the end of Section 1.5. We then express the frame components of the field tensor in retarded coordinates, in the form of an expansion in powers of $r$. This gives

$$
\begin{aligned}
F_{a 0}\left(u, r, \Omega^{a}\right) \equiv & F_{\alpha \beta}(x) e_{a}^{\alpha}(x) e_{0}^{\beta}(x) \\
= & \frac{e}{r^{2}} \Omega_{a}-\frac{e}{r}\left(a_{a}-a_{b} \Omega^{b} \Omega_{a}\right)+\frac{1}{3} e R_{b 0 c 0} \Omega^{b} \Omega^{c} \Omega_{a}-\frac{1}{6} e\left(5 R_{a 0 b 0} \Omega^{b}+R_{a b 0 c} \Omega^{b} \Omega^{c}\right) \\
& +\frac{1}{12} e\left(5 R_{00}+R_{b c} \Omega^{b} \Omega^{c}+R\right) \Omega_{a}+\frac{1}{3} e R_{a 0}-\frac{1}{6} e R_{a b} \Omega^{b}+F_{a 0}^{\mathrm{tail}}+\mathcal{O}(r), \\
F_{a b}\left(u, r, \Omega^{a}\right) \equiv & F_{\alpha \beta}(x) e_{a}^{\alpha}(x) e_{b}^{\beta}(x) \\
= & \frac{e}{r}\left(a_{a} \Omega_{b}-\Omega_{a} a_{b}\right)+\frac{1}{2} e\left(R_{a 0 b c}-R_{b 0 a c}+R_{a 0 c 0} \Omega_{b}-\Omega_{a} R_{b 0 c 0}\right) \Omega^{c} \\
& -\frac{1}{2} e\left(R_{a 0} \Omega_{b}-\Omega_{a} R_{b 0}\right)+F_{a b}^{\mathrm{tail}}+\mathcal{O}(r),
\end{aligned}
$$

where

$$
F_{a 0}^{\mathrm{tail}}=F_{\alpha^{\prime} \beta^{\prime}}^{\mathrm{tai}}\left(x^{\prime}\right) e_{a}^{\alpha^{\prime}} u^{\beta^{\prime}}, \quad F_{a b}^{\mathrm{tail}}=F_{\alpha^{\prime} \beta^{\prime}}^{\mathrm{tail}}\left(x^{\prime}\right) e_{a}^{\alpha^{\prime}} e_{b}^{\beta^{\prime}}
$$

are the frame components of the "tail part" of the field, which is given by

$$
F_{\alpha^{\prime} \beta^{\prime}}^{\text {tail }}\left(x^{\prime}\right)=2 e \int_{-\infty}^{u^{-}} \nabla_{\left[\alpha^{\prime}\right.} G_{\left.+\beta^{\prime}\right] \mu}\left(x^{\prime}, z\right) u^{\mu} d \tau
$$

In these expressions, all tensors (or their frame components) are evaluated at the retarded point $x^{\prime} \equiv z(u)$ associated with $x$; for example, $a_{a} \equiv a_{a}(u) \equiv a_{\alpha^{\prime}} e_{a}^{\alpha^{\prime}}$. The tail part of the electromagnetic field tensor is written as an integral over the portion of the world line that corresponds to the interval $-\infty<\tau \leq u^{-} \equiv u-0^{+}$; this represents the past history of the particle. The integral is cut short at $u^{-}$to avoid the singular behaviour of the retarded Green's function when $z(\tau)$ coincides with $x^{\prime}$; the portion of the Green's function involved in the tail integral is smooth, and the singularity at coincidence is completely accounted for by the other terms in Equations (23) and (24).

The expansion of $F_{\alpha \beta}(x)$ near the world line does indeed reveal many singular terms. We first recognize terms that diverge when $r \rightarrow 0$; for example the Coulomb field $F_{a 0}$ diverges as $r^{-2}$ when we approach the world line. But there are also terms that, though they stay bounded in the limit, possess a directional ambiguity at $r=0$; for example $F_{a b}$ contains a term proportional to $R_{a 0 b c} \Omega^{c}$ whose limit depends on the direction of approach.

This singularity structure is perfectly reproduced by the singular field $F_{\alpha \beta}^{\mathrm{S}}$ obtained from the potential

$$
A_{\mathrm{S}}^{\alpha}(x)=e \int_{\gamma} G_{\mathrm{S} \mu}^{\alpha}(x, z) u^{\mu} d \tau
$$


where $G_{\mathrm{S}}{ }_{\mu}^{\alpha}(x, z)$ is the singular Green's function of Equation (14). Near the world line the singular field is given by

$$
\begin{aligned}
F_{a 0}^{\mathrm{S}}\left(u, r, \Omega^{a}\right) \equiv & F_{\alpha \beta}^{\mathrm{S}}(x) e_{a}^{\alpha}(x) e_{0}^{\beta}(x) \\
= & \frac{e}{r^{2}} \Omega_{a}-\frac{e}{r}\left(a_{a}-a_{b} \Omega^{b} \Omega_{a}\right)-\frac{2}{3} e \dot{a}_{a}+\frac{1}{3} e R_{b 0 c 0} \Omega^{b} \Omega^{c} \Omega_{a}-\frac{1}{6} e\left(5 R_{a 0 b 0} \Omega^{b}+R_{a b 0 c} \Omega^{b} \Omega^{c}\right) \\
& +\frac{1}{12} e\left(5 R_{00}+R_{b c} \Omega^{b} \Omega^{c}+R\right) \Omega_{a}-\frac{1}{6} e R_{a b} \Omega^{b}+\mathcal{O}(r), \\
F_{a b}^{\mathrm{S}}\left(u, r, \Omega^{a}\right) \equiv & F_{\alpha \beta}^{\mathrm{S}}(x) e_{a}^{\alpha}(x) e_{b}^{\beta}(x) \\
= & \frac{e}{r}\left(a_{a} \Omega_{b}-\Omega_{a} a_{b}\right)+\frac{1}{2} e\left(R_{a 0 b c}-R_{b 0 a c}+R_{a 0 c 0} \Omega_{b}-\Omega_{a} R_{b 0 c 0}\right) \Omega^{c} \\
& -\frac{1}{2} e\left(R_{a 0} \Omega_{b}-\Omega_{a} R_{b 0}\right)+\mathcal{O}(r) .
\end{aligned}
$$

Comparison of these expressions with Equations (23) and (24) does indeed reveal that all singular terms are shared by both fields.

The difference between the retarded and singular fields defines the radiative field $F_{\alpha \beta}^{\mathrm{R}}(x)$. Its frame components are

$$
\begin{aligned}
F_{a 0}^{\mathrm{R}} & =\frac{2}{3} e \dot{a}_{a}+\frac{1}{3} e R_{a 0}+F_{a 0}^{\mathrm{tail}}+\mathcal{O}(r), \\
F_{a b}^{\mathrm{R}} & =F_{a b}^{\mathrm{tail}}+\mathcal{O}(r),
\end{aligned}
$$

and at $x^{\prime}$ the radiative field becomes

$$
F_{\alpha^{\prime} \beta^{\prime}}^{\mathrm{R}}=2 e u_{\left[\alpha^{\prime}\right.}\left(g_{\left.\beta^{\prime}\right] \gamma^{\prime}}+u_{\left.\beta^{\prime}\right]} u_{\gamma^{\prime}}\right)\left(\frac{2}{3} \dot{a}^{\gamma^{\prime}}+\frac{1}{3} R_{\delta^{\prime}}^{\gamma^{\prime}} u^{\delta^{\prime}}\right)+F_{\alpha^{\prime} \beta^{\prime}}^{\text {tail }},
$$

where $\dot{a}^{\gamma^{\prime}}=D a^{\gamma^{\prime}} / d \tau$ is the rate of change of the acceleration vector, and where the tail term was given by Equation (26). We see that $F_{\alpha \beta}^{\mathrm{R}}(x)$ is a smooth tensor field, even on the world line.

\subsection{Motion of an electric charge in curved spacetime}

I have argued in Section 1.4 that the self-force acting on a point electric charge is produced by the radiative field, and that the charge's equations of motion should take the form of $m a_{\mu}=$ $f_{\mu}^{\text {ext }}+e F_{\mu \nu}^{\mathrm{R}} u^{\nu}$, where $f_{\mu}^{\text {ext }}$ is an external force also acting on the particle. Substituting Equation (32) gives

$$
m a^{\mu}=f_{\mathrm{ext}}^{\mu}+e^{2}\left(\delta^{\mu}{ }_{\nu}+u^{\mu} u_{\nu}\right)\left(\frac{2}{3 m} \frac{D f_{\mathrm{ext}}^{\nu}}{d \tau}+\frac{1}{3} R_{\lambda}^{\nu} u^{\lambda}\right)+2 e^{2} u_{\nu} \int_{-\infty}^{\tau^{-}} \nabla^{[\mu} G_{+\lambda^{\prime}}^{\nu]}\left(z(\tau), z\left(\tau^{\prime}\right)\right) u^{\lambda^{\prime}} d \tau^{\prime}
$$

in which all tensors are evaluated at $z(\tau)$, the current position of the particle on the world line. The primed indices in the tail integral refer to a point $z\left(\tau^{\prime}\right)$ which represents a prior position; the integration is cut short at $\tau^{\prime}=\tau^{-} \equiv \tau-0^{+}$to avoid the singular behaviour of the retarded Green's function at coincidence. To get Equation (33) I have reduced the order of the differential equation by replacing $\dot{a}^{\nu}$ with $m^{-1} \dot{f}_{\text {ext }}^{\nu}$ on the right-hand side; this procedure was explained at the end of Section 1.2.

Equation (33) is the result that was first derived by DeWitt and Brehme [24] and later corrected by Hobbs [29]. (The original equation did not include the Ricci-tensor term.) In flat spacetime the Ricci tensor is zero, the tail integral disappears (because the Green's function vanishes everywhere within the domain of integration), and Equation (33) reduces to Dirac's result of Equation (5). In

Living Reviews in Relativity

http://www. livingreviews.org/lrr-2004-6 
curved spacetime the self-force does not vanish even when the electric charge is moving freely, in the absence of an external force: It is then given by the tail integral, which represents radiation emitted earlier and coming back to the particle after interacting with the spacetime curvature. This delayed action implies that, in general, the self-force is nonlocal in time: It depends not only on the current state of motion of the particle, but also on its past history. Lest this behaviour should seem mysterious, it may help to keep in mind that the physical process that leads to Equation (33) is simply an interaction between the charge and a free electromagnetic field $F_{\alpha \beta}^{\mathrm{R}}$; it is this field that carries the information about the charge's past.

\subsection{Motion of a scalar charge in curved spacetime}

The dynamics of a point scalar charge can be formulated in a way that stays fairly close to the electromagnetic theory. The particle's charge $q$ produces a scalar field $\Phi(x)$, which satisfies a wave equation

$$
(\square-\xi R) \Phi=-4 \pi \mu
$$

that is very similar to Equation (13). Here, $R$ is the spacetime's Ricci scalar, and $\xi$ is an arbitrary coupling constant; the scalar charge density $\mu(x)$ is given by a four-dimensional Dirac functional supported on the particle's world line $\gamma$. The retarded solution to the wave equation is

$$
\Phi(x)=q \int_{\gamma} G_{+}(x, z) d \tau,
$$

where $G_{+}(x, z)$ is the retarded Green's function associated with Equation (34). The field exerts a force on the particle, whose equations of motion are

$$
m a^{\mu}=q\left(g^{\mu \nu}+u^{\mu} u^{\nu}\right) \nabla_{\nu} \Phi,
$$

where $m$ is the particle's mass; this equation is very similar to the Lorentz-force law. But the dynamics of a scalar charge comes with a twist: If Equations (34) and (36) are to follow from a variational principle, the particle's mass should not be expected to be a constant of the motion. It is found instead to satisfy the differential equation

$$
\frac{d m}{d \tau}=-q u^{\mu} \nabla_{\mu} \Phi
$$

and in general $m$ will vary with proper time. This phenomenon is linked to the fact that a scalar field has zero spin: The particle can radiate monopole waves and the radiated energy can come at the expense of the rest mass.

The scalar field of Equation (35) diverges on the world line, and its singular part $\Phi_{\mathrm{S}}(x)$ must be removed before Equations (36) and (37) can be evaluated. This procedure produces the radiative field $\Phi_{\mathrm{R}}(x)$, and it is this field (which satisfies the homogeneous wave equation) that gives rise to a self-force. The gradient of the radiative field takes the form of

$$
\nabla_{\mu} \Phi_{\mathrm{R}}=-\frac{1}{12}(1-6 \xi) q R u_{\mu}+q\left(g_{\mu \nu}+u_{\mu} u_{\nu}\right)\left(\frac{1}{3} \dot{a}^{\nu}+\frac{1}{6} R_{\lambda}^{\nu} u^{\lambda}\right)+\Phi_{\mu}^{\mathrm{tail}}
$$

when it is evaluated of the world line. The last term is the tail integral

$$
\Phi_{\mu}^{\mathrm{tail}}=q \int_{-\infty}^{\tau^{-}} \nabla_{\mu} G_{+}\left(z(\tau), z\left(\tau^{\prime}\right)\right) d \tau^{\prime}
$$

and this brings the dependence on the particle's past. 
Substitution of Equation (38) into Equations (36) and (37) gives the equations of motion of a point scalar charge. (At this stage I introduce an external force $f_{\text {ext }}^{\mu}$ and reduce the order of the differential equation.) The acceleration is given by

$$
m a^{\mu}=f_{\mathrm{ext}}^{\mu}+q^{2}\left(\delta^{\mu}{ }_{\nu}+u^{\mu} u_{\nu}\right)\left[\frac{1}{3 m} \frac{D f_{\mathrm{ext}}^{\nu}}{d \tau}+\frac{1}{6} R_{\lambda}^{\nu} u^{\lambda}+\int_{-\infty}^{\tau^{-}} \nabla^{\nu} G_{+}\left(z(\tau), z\left(\tau^{\prime}\right)\right) d \tau^{\prime}\right],
$$

and the mass changes according to

$$
\frac{d m}{d \tau}=-\frac{1}{12}(1-6 \xi) q^{2} R-q^{2} u^{\mu} \int_{-\infty}^{\tau^{-}} \nabla_{\mu} G_{+}\left(z(\tau), z\left(\tau^{\prime}\right)\right) d \tau^{\prime} .
$$

These equations were first derived by Quinn $[48]^{2}$.

In flat spacetime the Ricci-tensor term and the tail integral disappear, and Equation (40) takes the form of Equation (5) with $q^{2} /(3 m)$ replacing the factor of $2 e^{2} /(3 m)$. In this simple case Equation (41) reduces to $d m / d \tau=0$ and the mass is in fact a constant. This property remains true in a conformally-flat spacetime when the wave equation is conformally invariant $(\xi=1 / 6)$ : In this case the Green's function possesses only a light-cone part, and the right-hand side of Equation (41) vanishes. In generic situations the mass of a point scalar charge will vary with proper time.

\subsection{Motion of a point mass, or a black hole, in a background spacetime}

The case of a point mass moving in a specified background spacetime presents itself with a serious conceptual challenge, as the fundamental equations of the theory are nonlinear and the very notion of a "point mass" is somewhat misguided. Nevertheless, to the extent that the perturbation $h_{\alpha \beta}(x)$ created by the point mass can be considered to be "small", the problem can be formulated in close analogy with what was presented before.

We take the metric $g_{\alpha \beta}$ of the background spacetime to be a solution of the Einstein field equations in vacuum. (We impose this condition globally.) We describe the gravitational perturbation produced by a point particle of mass $m$ in terms of trace-reversed potentials $\gamma_{\alpha \beta}$ defined by

$$
\gamma_{\alpha \beta}=h_{\alpha \beta}-\frac{1}{2}\left(g^{\gamma \delta} h_{\gamma \delta}\right) g_{\alpha \beta}
$$

where $h_{\alpha \beta}$ is the difference between $\mathrm{g}_{\alpha \beta}$, the actual metric of the perturbed spacetime, and $g_{\alpha \beta}$. The potentials satisfy the wave equation

$$
\square \gamma^{\alpha \beta}+2 R_{\gamma \delta}^{\alpha \beta} \gamma^{\gamma \delta}=-16 \pi T^{\alpha \beta}
$$

together with the Lorenz gauge condition $\gamma_{; \beta}^{\alpha \beta}=0$. Here and below, covariant differentiation refers to a connection that is compatible with the background metric, $\square=g^{\alpha \beta} \nabla_{\alpha} \nabla_{\beta}$ is the wave operator for the background spacetime, and $T^{\alpha \beta}$ is the stress-energy tensor of the point mass; this is given by a Dirac distribution supported on the particle's world line $\gamma$. The retarded solution is

$$
\gamma^{\alpha \beta}(x)=4 m \int_{\gamma} G_{+\mu \nu}^{\alpha \beta}(x, z) u^{\mu} u^{\nu} d \tau
$$

where $G_{+\mu \nu}^{\alpha \beta}(x, z)$ is the retarded Green's function associated with Equation (43). The perturbation $h_{\alpha \beta}(x)$ can be recovered by inverting Equation (42).

\footnotetext{
${ }^{2} \mathrm{His}$ analysis was restricted to a minimally-coupled scalar field, so that $\xi=0$ in his expressions. The extension to an arbitrary coupling constant was carried out by myself for this review.
}

Living Reviews in Relativity

http: //www. livingreviews. org/lrr-2004-6 
Equations of motion for the point mass can be obtained by formally demanding that the motion be geodesic in the perturbed spacetime with metric $\mathrm{g}_{\alpha \beta}=g_{\alpha \beta}+h_{\alpha \beta}$. After a mapping to the background spacetime, the equations of motion take the form of

$$
a^{\mu}=-\frac{1}{2}\left(g^{\mu \nu}+u^{\mu} u^{\nu}\right)\left(2 h_{\nu \lambda ; \rho}-h_{\lambda \rho ; \nu}\right) u^{\lambda} u^{\rho} .
$$

The acceleration is thus proportional to $m$; in the test-mass limit the world line of the particle is a geodesic of the background spacetime.

We now remove $h_{\alpha \beta}^{\mathrm{S}}(x)$ from the retarded perturbation and postulate that it is the radiative field $h_{\alpha \beta}^{\mathrm{S}}(x)$ that should act on the particle. (Note that $\gamma_{\alpha \beta}^{\mathrm{S}}$ satisfies the same wave equation as the retarded potentials, but that $\gamma_{\alpha \beta}^{\mathrm{R}}$ is a free gravitational field that satisfies the homogeneous wave equation.) On the world line we have

$$
h_{\mu \nu ; \lambda}^{\mathrm{R}}=-4 m\left(u_{(\mu} R_{\nu) \rho \lambda \xi}+R_{\mu \rho \nu \xi} u_{\lambda}\right) u^{\rho} u^{\xi}+h_{\mu \nu \lambda}^{\mathrm{tail}},
$$

where the tail term is given by

$$
h_{\mu \nu \lambda}^{\mathrm{tail}}=4 m \int_{-\infty}^{\tau^{-}} \nabla_{\lambda}\left(G_{+\mu \nu \mu^{\prime} \nu^{\prime}}-\frac{1}{2} g_{\mu \nu} G_{+\rho \mu^{\prime} \nu^{\prime}}{ }^{\rho}\right)\left(z(\tau), z\left(\tau^{\prime}\right)\right) u^{\mu^{\prime}} u^{\nu^{\prime}} d \tau^{\prime} .
$$

When Equation (46) is substituted into Equation (45) we find that the terms that involve the Riemann tensor cancel out, and we are left with

$$
a^{\mu}=-\frac{1}{2}\left(g^{\mu \nu}+u^{\mu} u^{\nu}\right)\left(2 h_{\nu \lambda \rho}^{\mathrm{tail}}-h_{\lambda \rho \nu}^{\mathrm{tail}}\right) u^{\lambda} u^{\rho} .
$$

Only the tail integral appears in the final form of the equations of motion. It involves the current position $z(\tau)$ of the particle, at which all tensors with unprimed indices are evaluated, as well as all prior positions $z\left(\tau^{\prime}\right)$, at which tensors with primed indices are evaluated. As before the integral is cut short at $\tau^{\prime}=\tau^{-} \equiv \tau-0^{+}$to avoid the singular behaviour of the retarded Green's function at coincidence.

The equations of motion of Equation (48) were first derived by Mino, Sasaki, and Tanaka [39], and then reproduced with a different analysis by Quinn and Wald [49]. They are now known as the MiSaTaQuWa equations of motion. Detweiler and Whiting [23] have contributed the compelling interpretation that the motion is actually geodesic in a spacetime with metric $g_{\alpha \beta}+h_{\alpha \beta}^{\mathrm{R}}$. This metric satisfies the Einstein field equations in vacuum and is perfectly smooth on the world line. This spacetime can thus be viewed as the background spacetime perturbed by a free gravitational wave produced by the particle at an earlier stage of its history.

While Equation (48) does indeed give the correct equations of motion for a small mass $m$ moving in a background spacetime with metric $g_{\alpha \beta}$, the derivation outlined here leaves much to be desired - to what extent should we trust an analysis based on the existence of a point mass? Fortunately, Mino, Sasaki, and Tanaka [39] gave two different derivations of their result, and the second derivation was concerned not with the motion of a point mass, but with the motion of a small nonrotating black hole. In this alternative derivation of the MiSaTaQuWa equations, the metric of the black hole perturbed by the tidal gravitational field of the external universe is matched to the metric of the background spacetime perturbed by the moving black hole. Demanding that this metric be a solution to the vacuum field equations determines the motion of the black hole: It must move according to Equation (48). This alternative derivation is entirely free of conceptual and technical pitfalls, and we conclude that the MiSaTaQuWa equations can be trusted to describe the motion of any gravitating body in a curved background spacetime (so long as the body's internal structure can be ignored). 
It is important to understand that unlike Equations (33) and (40), which are true tensorial equations, Equation (48) reflects a specific choice of coordinate system and its form would not be preserved under a coordinate transformation. In other words, the MiSaTaQuWa equations are not gauge invariant, and they depend upon the Lorenz gauge condition $\gamma_{; \beta}^{\alpha \beta}=0$. Barack and Ori [8] have shown that under a coordinate transformation of the form $x^{\alpha} \rightarrow x^{\alpha}+\xi^{\alpha}$, where $x^{\alpha}$ are the coordinates of the background spacetime and $\xi^{\alpha}$ is a smooth vector field of order $m$, the particle's acceleration changes according to $a^{\mu} \rightarrow a^{\mu}+a[\xi]^{\mu}$, where

$$
a[\xi]^{\mu}=\left(\delta_{\nu}^{\mu}+u^{\mu} u_{\nu}\right)\left(\frac{D^{2} \xi^{\nu}}{d \tau^{2}}+R_{\rho \omega \lambda}^{\nu} u^{\rho} \xi^{\omega} u^{\lambda}\right)
$$

is the "gauge acceleration"; $D^{2} \xi^{\nu} / d \tau^{2}=\left(\xi_{; \mu}^{\nu} u^{\mu}\right)_{; \rho} u^{\rho}$ is the second covariant derivative of $\xi^{\nu}$ in the direction of the world line. This implies that the particle's acceleration can be altered at will by a gauge transformation; $\xi^{\alpha}$ could even be chosen so as to produce $a^{\mu}=0$, making the motion geodesic after all. This observation provides a dramatic illustration of the following point: The MiSaTaQuWa equations of motion are not gauge invariant and they cannot by themselves produce a meaningful answer to a well-posed physical question; to obtain such answers it shall always be necessary to combine the equations of motion with the metric perturbation $h_{\alpha \beta}$ so as to form gaugeinvariant quantities that will correspond to direct observables. This point is very important and cannot be over-emphasized.

\subsection{Evaluation of the self-force}

To concretely evaluate the self-force, whether it be for a scalar charge, an electric charge, or a point mass, is a difficult undertaking. The difficulty resides mostly with the computation of the retarded Green's function for the spacetime under consideration. Because Green's functions are known for a very limited number of spacetimes, the self-force has so far been evaluated in a rather limited number of situations.

The first evaluation of the electromagnetic self-force was carried out by DeWitt and DeWitt [41] for a charge moving freely in a weakly-curved spacetime characterized by a Newtonian potential $\Phi \ll 1$. (This condition must be imposed globally, and requires the spacetime to contain a matter distribution.) In this context the right-hand side of Equation (33) reduces to the tail integral, since there is no external force acting on the charge. They found the spatial components of the self-force to be given by

$$
\boldsymbol{f}_{\mathrm{em}}=e^{2} \frac{M}{r^{3}} \hat{\boldsymbol{r}}+\frac{2}{3} e^{2} \frac{d \boldsymbol{g}}{d t},
$$

where $M$ is the total mass contained in the spacetime, $r=|\boldsymbol{x}|$ is the distance from the centre of mass, $\hat{\boldsymbol{r}}=\boldsymbol{x} / r$, and $\boldsymbol{g}=-\boldsymbol{\nabla} \Phi$ is the Newtonian gravitational field. (In these expressions the boldfaced symbols represent vectors in three-dimensional flat space.) The first term on the right-hand side of Equation (50) is a conservative correction to the Newtonian force $\boldsymbol{m g}$. The second term is the standard radiation-reaction force; although it comes from the tail integral, this is the same result that would be obtained in flat spacetime if an external force $\boldsymbol{m g}$ were acting on the particle. This agreement is necessary, but remarkable!

A similar expression was obtained by Pfenning and Poisson [46] for the case of a scalar charge. Here

$$
\boldsymbol{f}_{\text {scalar }}=2 \xi q^{2} \frac{M}{r^{3}} \hat{\boldsymbol{r}}+\frac{1}{3} q^{2} \frac{d \boldsymbol{g}}{d t},
$$

where $\xi$ is the coupling of the scalar field to the spacetime curvature; the conservative term disappears when the field is minimally coupled. Pfenning and Poisson also computed the gravitational self-force acting on a massive particle moving in a weakly curved spacetime. The expression they

Living Reviews in Relativity

http://www. livingreviews.org/lrr-2004-6 
obtained is in complete agreement (within its domain of validity) with the standard post-Newtonian equations of motion.

The force required to hold an electric charge in place in a Schwarzschild spacetime was computed, without approximations, by Smith and Will [54]. As measured by a free-falling observer momentarily at rest at the position of the charge, the total force is

$$
f=\frac{M m}{r^{2}}\left(1-\frac{2 M}{r}\right)^{-1 / 2}-e^{2} \frac{M}{r^{3}},
$$

and it is directed in the radial direction. Here, $m$ is the mass of the charge, $M$ is the mass of the black hole, and $r$ is the charge's radial coordinate (the expression is valid in Schwarzschild coordinates). The first term on the right-hand side of Equation (52) is the force required to keep a neutral test particle stationary in a Schwarzschild spacetime; the second term is the negative of the electromagnetic self-force, and its expression agrees with the weak-field result of Equation (50). Wiseman [62] performed a similar calculation for a scalar charge. He found that in this case the self-force vanishes. This result is not incompatible with Equation (51), even for nonminimal coupling, because the computation of the weak-field self-force requires the presence of matter, while Wiseman's scalar charge lives in a purely vacuum spacetime.

The intriguing phenomenon of mass loss by a scalar charge was studied by Burko, Harte, and Poisson [15] in the simple context of a particle at rest in an expanding universe. For the special cases of a de Sitter cosmology, or a spatially-flat matter-dominated universe, the retarded Green's function could be computed, and the action of the scalar field on the particle determined, without approximations. In de Sitter spacetime the particle is found to radiate all of its rest mass into monopole scalar waves. In the matter-dominated cosmology this happens only if the charge of the particle is sufficiently large; for smaller charges the particle first loses a fraction of its mass, but then regains it eventually.

In recent years a large effort has been devoted to the elaboration of a practical method to compute the (scalar, electromagnetic, and gravitational) self-force in the Schwarzschild spacetime. This work originated with Barack and Ori [7] and was pursued by Barack [2, 3] until it was put in its definitive form by Barack, Mino, Nakano, Ori, and Sasaki [6, 9, 11, 38]. The idea is to take advantage of the spherical symmetry of the Schwarzschild solution by decomposing the retarded Green's function $G_{+}\left(x, x^{\prime}\right)$ into spherical-harmonic modes which can be computed individually. (To be concrete I refer here to the scalar case, but the method works just as well for the electromagnetic and gravitational cases.) From the mode-decomposition of the Green's function one obtains a mode-decomposition of the field gradient $\nabla_{\alpha} \Phi$, and from this subtracts a mode-decomposition of the singular field $\nabla_{\alpha} \Phi_{\mathrm{S}}$, for which a local expression is known. This results in the radiative field $\nabla_{\alpha} \Phi_{\mathrm{R}}$ decomposed into modes, and since this field is well behaved on the world line, it can be directly evaluated at the position of the particle by summing over all modes. (This sum converges because the radiative field is smooth; the mode sums for the retarded or singular fields, on the other hand, do not converge.) An extension of this method to the Kerr spacetime has recently been presented [44, 34, 10], and Mino [37] has devised a surprisingly simple prescription to calculate the time-averaged evolution of a generic orbit around a Kerr black hole.

The mode-sum method was applied to a number of different situations. Burko computed the self-force acting on an electric charge in circular motion in flat spacetime [12], as well as on a scalar and electric charge kept stationary in a Schwarzschild spacetime [14], in a spacetime that contains a spherical matter shell (Burko, Liu, and Soren [17]), and in a Kerr spacetime (Burko and Liu [16]). Burko also computed the scalar self-force acting on a particle in circular motion around a Schwarzschild black hole [13], a calculation that was recently revisited by Detweiler, Messaritaki, and Whiting [21]. Barack and Burko considered the case of a particle falling radially into a Schwarzschild black hole, and evaluated the scalar self-force acting on such a particle [4]; Lousto [33] and Barack and Lousto [5], on the other hand, calculated the gravitational self-force. 


\subsection{Organization of this review}

The main body of the review begins in Section 2 with a description of the general theory of bitensors, the name designating tensorial functions of two points in spacetime. I introduce Synge's world function $\sigma\left(x, x^{\prime}\right)$ and its derivatives in Section 2.1, the parallel propagator $g_{\alpha^{\prime}}^{\alpha}\left(x, x^{\prime}\right)$ in Section 2.3, and the van Vleck determinant $\Delta\left(x, x^{\prime}\right)$ in Section 2.5. An important portion of the theory (covered in Sections 2.2 and 2.4) is concerned with the expansion of bitensors when $x$ is very close to $x^{\prime}$; expansions such as those displayed in Equations (23) and (24) are based on these techniques. The presentation in Section 2 borrows heavily from Synge's book [55] and the article by DeWitt and Brehme [24]. These two sources use different conventions for the Riemann tensor, and I have adopted Synge's conventions (which agree with those of Misner, Thorne, and Wheeler [40]). The reader is therefore warned that formulae derived in Section 2 may look superficially different from what can be found in DeWitt and Brehme.

In Section 3 I introduce a number of coordinate systems that play an important role in later parts of the review. As a warmup exercise I first construct (in Section 3.1) Riemann normal coordinates in a neighbourhood of a reference point $x^{\prime}$. I then move on (in Section 3.2) to Fermi normal coordinates [36], which are defined in a neighbourhood of a world line $\gamma$. The retarded coordinates, which are also based at a world line and which were briefly introduced in Section 1.5, are covered systematically in Section 3.3. The relationship between Fermi and retarded coordinates is worked out in Section 3.4, which also locates the advanced point $z(v)$ associated with a field point $x$. The presentation in Section 3 borrows heavily from Synge's book [55]. In fact, I am much indebted to Synge for initiating the construction of retarded coordinates in a neighbourhood of a world line. I have implemented his program quite differently (Synge was interested in a large neighbourhood of the world line in a weakly curved spacetime, while I am interested in a small neighbourhood in a strongly curved spacetime), but the idea is originally his.

In Section 4 I review the theory of Green's functions for (scalar, vectorial, and tensorial) wave equations in curved spacetime. I begin in Section 4.1 with a pedagogical introduction to the retarded and advanced Green's functions for a massive scalar field in flat spacetime; in this simple context the all-important Hadamard decomposition [28] of the Green's function into "light-cone" and "tail" parts can be displayed explicitly. The invariant Dirac functional is defined in Section 4.2 along with its restrictions on the past and future null cones of a reference point $x^{\prime}$. The retarded, advanced, singular, and radiative Green's functions for the scalar wave equation are introduced in Section 4.3. In Sections 4.4 and 4.5 I cover the vectorial and tensorial wave equations, respectively. The presentation in Section 4 is based partly on the paper by DeWitt and Brehme [24], but it is inspired mostly by Friedlander's book [27]. The reader should be warned that in one important aspect, my notation differs from the notation of DeWitt and Brehme: While they denote the tail part of the Green's function by $-v\left(x, x^{\prime}\right)$, I have taken the liberty of eliminating the silly minus sign and I call it instead $+V\left(x, x^{\prime}\right)$. The reader should also note that all my Green's functions are normalized in the same way, with a factor of $-4 \pi$ multiplying a four-dimensional Dirac functional of the right-hand side of the wave equation. (The gravitational Green's function is sometimes normalized with a $-16 \pi$ on the right-hand side.)

In Section 5 I compute the retarded, singular, and radiative fields associated with a point scalar charge (Section 5.1), a point electric charge (Section 5.2), and a point mass (Section 5.3). I provide two different derivations for each of the equations of motion. The first type of derivation was outlined previously: I follow Detweiler and Whiting [23] and postulate that only the radiative field exerts a force on the particle. In the second type of derivation I take guidance from Quinn and Wald [49] and postulate that the net force exerted on a point particle is given by an average of the retarded field over a surface of constant proper distance orthogonal to the world line - this restframe average is easily carried out in Fermi normal coordinates. The averaged field is still infinite on the world line, but the divergence points in the direction of the acceleration vector and it can

Living Reviews in Relativity

http://www . livingreviews . org/lrr-2004-6 
thus be removed by mass renormalization. Such calculations show that while the singular field does not affect the motion of the particle, it nonetheless contributes to its inertia. In Section 5.4 I present an alternative derivation of the MiSaTaQuWa equations of motion based on the method of matched asymptotic expansions [35, 31, 58, 19, 1, 20]; the derivation applies to a small nonrotating black hole instead of a point mass. The ideas behind this derivation were contained in the original paper by Mino, Sasaki, and Tanaka [39], but the implementation given here, which involves the retarded coordinates of Section 3.3 and displays explicitly the transformation between external and internal coordinates, is original work.

Concluding remarks are presented in Section 5.5. Throughout this review I use geometrized units and adopt the notations and conventions of Misner, Thorne, and Wheeler [40]. 


\section{General Theory of Bitensors}

\subsection{Synge's world function}

\subsubsection{Definition}

In this and the following sections we will construct a number of bitensors, tensorial functions of two points in spacetime. The first is $x^{\prime}$, to which we refer as the "base point", and to which we assign indices $\alpha^{\prime}, \beta^{\prime}$, etc. The second is $x$, to which we refer as the "field point", and to which we assign indices $\alpha, \beta$, etc. We assume that $x$ belongs to $\mathcal{N}\left(x^{\prime}\right)$, the normal convex neighbourhood of $x^{\prime}$; this is the set of points that are linked to $x^{\prime}$ by a unique geodesic. The geodesic $\beta$ that links $x$ to $x^{\prime}$ is described by relations $z^{\mu}(\lambda)$ in which $\lambda$ is an affine parameter that ranges from $\lambda_{0}$ to $\lambda_{1}$; we have $z\left(\lambda_{0}\right) \equiv x^{\prime}$ and $z\left(\lambda_{1}\right) \equiv x$. To an arbitrary point $z$ on the geodesic we assign indices $\mu, \nu$, etc. The vector $t^{\mu}=d z^{\mu} / d \lambda$ is tangent to the geodesic, and it obeys the geodesic equation $D t^{\mu} / d \lambda=0$. The situation is illustrated in Figure 5 .

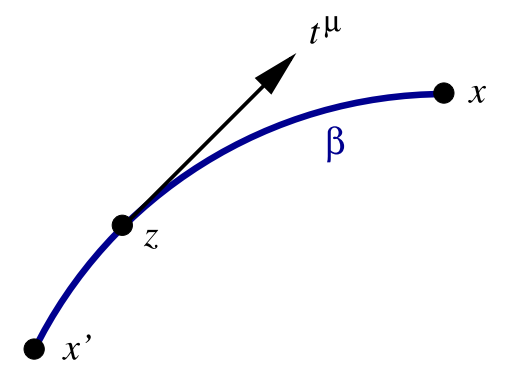

Figure 5: The base point $x^{\prime}$, the field point $x$, and the geodesic $\beta$ that links them. The geodesic is described by parametric relations $z^{\mu}(\lambda)$, and $t^{\mu}=d z^{\mu} / d \lambda$ is its tangent vector.

Synge's world function is a scalar function of the base point $x^{\prime}$ and the field point $x$. It is defined by

$$
\sigma\left(x, x^{\prime}\right)=\frac{1}{2}\left(\lambda_{1}-\lambda_{0}\right) \int_{\lambda_{0}}^{\lambda_{1}} g_{\mu \nu}(z) t^{\mu} t^{\nu} d \lambda
$$

and the integral is evaluated on the geodesic $\beta$ that links $x$ to $x^{\prime}$. You may notice that $\sigma$ is invariant under a constant rescaling of the affine parameter, $\lambda \rightarrow \bar{\lambda}=a \lambda+b$, where $a$ and $b$ are constants.

By virtue of the geodesic equation, the quantity $\varepsilon \equiv g_{\mu \nu} t^{\mu} t^{\nu}$ is constant on the geodesic. The world function is therefore numerically equal to $\frac{1}{2} \varepsilon\left(\lambda_{1}-\lambda_{0}\right)^{2}$. If the geodesic is timelike, then $\lambda$ can be set equal to the proper time $\tau$, which implies that $\varepsilon=-1$ and $\sigma=-\frac{1}{2}(\Delta \tau)^{2}$. If the geodesic is spacelike, then $\lambda$ can be set equal to the proper distance $s$, which implies that $\varepsilon=1$ and $\sigma=\frac{1}{2}(\Delta s)^{2}$. If the geodesic is null, then $\sigma=0$. Quite generally, therefore, the world function is half the squared geodesic distance between the points $x^{\prime}$ and $x$.

In flat spacetime, the geodesic linking $x$ to $x^{\prime}$ is a straight line, and $\sigma=\frac{1}{2} \eta_{\alpha \beta}\left(x-x^{\prime}\right)^{\alpha}\left(x-x^{\prime}\right)^{\beta}$ in Lorentzian coordinates.

\subsubsection{Differentiation of the world function}

The world function $\sigma\left(x, x^{\prime}\right)$ can be differentiated with respect to either argument. We let $\sigma_{\alpha}=$ $\partial \sigma / \partial x^{\alpha}$ be its partial derivative with respect to $x$, and $\sigma_{\alpha^{\prime}}=\partial \sigma / \partial x^{\alpha^{\prime}}$ its partial derivative with respect to $x^{\prime}$. It is clear that $\sigma_{\alpha}$ behaves as a dual vector with respect to tensorial operations carried out at $x$, but as a scalar with respect to operations carried out $x^{\prime}$. Similarly, $\sigma_{\alpha^{\prime}}$ is a scalar at $x$ but a dual vector at $x^{\prime}$.

Living Reviews in Relativity

http://www. livingreviews . org/lrr-2004-6 
We let $\sigma_{\alpha \beta} \equiv \nabla_{\beta} \sigma_{\alpha}$ be the covariant derivative of $\sigma_{\alpha}$ with respect to $x$; this is a rank-2 tensor at $x$ and a scalar at $x^{\prime}$. Because $\sigma$ is a scalar at $x$, we have that this tensor is symmetric: $\sigma_{\beta \alpha}=\sigma_{\alpha \beta}$. Similarly, we let $\sigma_{\alpha \beta^{\prime}} \equiv \partial_{\beta^{\prime}} \sigma_{\alpha}=\partial^{2} \sigma / \partial x^{\beta^{\prime}} \partial x^{\alpha}$ be the partial derivative of $\sigma_{\alpha}$ with respect to $x^{\prime}$; this is a dual vector both at $x$ and $x^{\prime}$. We can also define $\sigma_{\alpha^{\prime} \beta} \equiv \partial_{\beta} \sigma_{\alpha^{\prime}}=\partial^{2} \sigma / \partial x^{\beta} \partial x^{\alpha^{\prime}}$ to be the partial derivative of $\sigma_{\alpha^{\prime}}$ with respect to $x$. Because partial derivatives commute, these bitensors are equal: $\sigma_{\beta^{\prime} \alpha}=\sigma_{\alpha \beta^{\prime}}$. Finally, we let $\sigma_{\alpha^{\prime} \beta^{\prime}} \equiv \nabla_{\beta^{\prime}} \sigma_{\alpha^{\prime}}$ be the covariant derivative of $\sigma_{\alpha^{\prime}}$ with respect to $x^{\prime}$; this is a symmetric rank-2 tensor at $x^{\prime}$ and a scalar at $x$.

The notation is easily extended to any number of derivatives. For example, we let $\sigma_{\alpha \beta \gamma \delta^{\prime}} \equiv$ $\nabla_{\delta^{\prime}} \nabla_{\gamma} \nabla_{\beta} \nabla_{\alpha} \sigma$, which is a rank-3 tensor at $x$ and a dual vector at $x^{\prime}$. This bitensor is symmetric in the pair of indices $\alpha$ and $\beta$, but not in the pairs $\alpha$ and $\gamma$, nor $\beta$ and $\gamma$. Because $\nabla_{\delta^{\prime}}$ is here an ordinary partial derivative with respect to $x^{\prime}$, the bitensor is symmetric in any pair of indices involving $\delta^{\prime}$. The ordering of the primed index relative to the unprimed indices is therefore irrelevant: The same bitensor can be written as $\sigma_{\delta^{\prime} \alpha \beta \gamma}$ or $\sigma_{\alpha \delta^{\prime} \beta \gamma}$ or $\sigma_{\alpha \beta \delta^{\prime} \gamma}$, making sure that the ordering of the unprimed indices is not altered.

More generally, we can show that derivatives of any bitensor $\Omega_{\ldots}\left(x, x^{\prime}\right)$ satisfy the property

$$
\Omega_{\ldots ; \beta \alpha^{\prime} \ldots}=\Omega_{\ldots ; \alpha^{\prime} \beta \ldots}
$$

in which "..." stands for any combination of primed and unprimed indices. We start by establishing the symmetry of $\Omega_{\ldots ; \alpha \beta^{\prime}}$ with respect to the pair $\alpha$ and $\beta^{\prime}$. This is most easily done by adopting Fermi normal coordinates (see Section 3.2) adapted to the geodesic $\beta$, and setting the connection to zero both at $x$ and $x^{\prime}$. In these coordinates, the bitensor $\Omega_{\ldots ; \alpha}$ is the partial derivative of $\Omega_{\ldots}$ with respect to $x^{\alpha}$, and $\Omega_{\ldots ; \alpha \beta^{\prime}}$ is obtained by taking an additional partial derivative with respect to $x^{\beta^{\prime}}$. These two operations commute, and $\Omega_{\ldots ; \beta^{\prime} \alpha}=\Omega_{\ldots ; \alpha \beta^{\prime}}$ follows as a bitensorial identity. Equation (54) then follows by further differentiation with respect to either $x$ or $x^{\prime}$.

The message of Equation (54), when applied to derivatives of the world function, is that while the ordering of the primed and unprimed indices relative to themselves is important, their ordering with respect to each other is arbitrary. For example, $\sigma_{\alpha^{\prime} \beta^{\prime} \gamma \delta^{\prime} \epsilon}=\sigma_{\alpha^{\prime} \beta^{\prime} \delta^{\prime} \gamma \epsilon}=\sigma_{\gamma \epsilon \alpha^{\prime} \beta^{\prime} \delta^{\prime}}$.

\subsubsection{Evaluation of first derivatives}

We can compute $\sigma_{\alpha}$ by examining how $\sigma$ varies when the field point $x$ moves. We let the new field point be $x+\delta x$, and $\delta \sigma \equiv \sigma\left(x+\delta x, x^{\prime}\right)-\sigma\left(x, x^{\prime}\right)$ is the corresponding variation of the world function. We let $\beta+\delta \beta$ be the unique geodesic that links $x+\delta x$ to $x^{\prime}$; it is described by relations $z^{\mu}(\lambda)+\delta z^{\mu}(\lambda)$, in which the affine parameter is scaled in such a way that it runs from $\lambda_{0}$ to $\lambda_{1}$ also on the new geodesic. We note that $\delta z\left(\lambda_{0}\right)=\delta x^{\prime} \equiv 0$ and $\delta z\left(\lambda_{1}\right)=\delta x$.

Working to first order in the variations, Equation (53) implies

$$
\delta \sigma=\Delta \lambda \int_{\lambda_{0}}^{\lambda_{1}}\left(g_{\mu \nu} \dot{z}^{\mu} \delta \dot{z}^{\nu}+\frac{1}{2} g_{\mu \nu, \lambda} \dot{z}^{\mu} \dot{z}^{\nu} \delta z^{\lambda}\right) d \lambda,
$$

where $\Delta \lambda=\lambda_{1}-\lambda_{0}$, an overdot indicates differentiation with respect to $\lambda$, and the metric and its derivatives are evaluated on $\beta$. Integrating the first term by parts gives

$$
\delta \sigma=\Delta \lambda\left[g_{\mu \nu} \dot{z}^{\mu} \delta z^{\nu}\right]_{\lambda_{0}}^{\lambda_{1}}-\Delta \lambda \int_{\lambda_{0}}^{\lambda_{1}}\left(g_{\mu \nu} \ddot{z}^{\nu}+\Gamma_{\mu \nu \lambda} \dot{z}^{\nu} \dot{z}^{\lambda}\right) \delta z^{\mu} d \lambda .
$$

The integral vanishes because $z^{\mu}(\lambda)$ satisfies the geodesic equation. The boundary term at $\lambda_{0}$ is zero because the variation $\delta z^{\mu}$ vanishes there. We are left with $\delta \sigma=\Delta \lambda g_{\alpha \beta} t^{\alpha} \delta x^{\beta}$, or

$$
\sigma_{\alpha}\left(x, x^{\prime}\right)=\left(\lambda_{1}-\lambda_{0}\right) g_{\alpha \beta} t^{\beta},
$$


in which the metric and the tangent vector are both evaluated at $x$. Apart from a factor $\Delta \lambda$, we see that $\sigma^{\alpha}\left(x, x^{\prime}\right)$ is equal to the geodesic's tangent vector at $x$. If in Equation (55) we replace $x$ by a generic point $z(\lambda)$ on $\beta$, and if we correspondingly replace $\lambda_{1}$ by $\lambda$, we obtain $\sigma^{\mu}\left(z, x^{\prime}\right)=\left(\lambda-\lambda_{0}\right) t^{\mu}$; we therefore see that $\sigma^{\mu}\left(z, x^{\prime}\right)$ is a rescaled tangent vector on the geodesic.

A virtually identical calculation reveals how $\sigma$ varies under a change of base point $x^{\prime}$. Here the variation of the geodesic is such that $\delta z\left(\lambda_{0}\right)=\delta x^{\prime}$ and $\delta z\left(\lambda_{1}\right)=\delta x=0$, and we obtain $\delta \sigma=-\Delta \lambda g_{\alpha^{\prime} \beta^{\prime}} t^{\alpha^{\prime}} \delta x^{\beta^{\prime}}$. This shows that

$$
\sigma_{\alpha^{\prime}}\left(x, x^{\prime}\right)=-\left(\lambda_{1}-\lambda_{0}\right) g_{\alpha^{\prime} \beta^{\prime}} t^{\beta^{\prime}}
$$

in which the metric and the tangent vector are both evaluated at $x^{\prime}$. Apart from a factor $\Delta \lambda$, we see that $\sigma^{\alpha^{\prime}}\left(x, x^{\prime}\right)$ is minus the geodesic's tangent vector at $x^{\prime}$.

It is interesting to compute the norm of $\sigma_{\alpha}$. According to Equation (55) we have $g_{\alpha \beta} \sigma^{\alpha} \sigma^{\beta}=$ $(\Delta \lambda)^{2} g_{\alpha \beta} t^{\alpha} t^{\beta}=(\Delta \lambda)^{2} \varepsilon$. According to Equation (53), this is equal to $2 \sigma$. We have obtained

$$
g^{\alpha \beta} \sigma_{\alpha} \sigma_{\beta}=2 \sigma,
$$

and similarly

$$
g^{\alpha^{\prime} \beta^{\prime}} \sigma_{\alpha^{\prime}} \sigma_{\beta^{\prime}}=2 \sigma \text {. }
$$

These important relations will be the starting point of many computations to be described below.

We note that in flat spacetime, $\sigma_{\alpha}=\eta_{\alpha \beta}\left(x-x^{\prime}\right)^{\beta}$ and $\sigma_{\alpha^{\prime}}=-\eta_{\alpha \beta}\left(x-x^{\prime}\right)^{\beta}$ in Lorentzian coordinates. From this it follows that $\sigma_{\alpha \beta}=\sigma_{\alpha^{\prime} \beta^{\prime}}=-\sigma_{\alpha \beta^{\prime}}=-\sigma_{\alpha^{\prime} \beta}=\eta_{\alpha \beta}$, and finally, $g^{\alpha \beta} \sigma_{\alpha \beta}=$ $4=g^{\alpha^{\prime} \beta^{\prime}} \sigma_{\alpha^{\prime} \beta^{\prime}}$.

\subsubsection{Congruence of geodesics emanating from $x^{\prime}$}

If the base point $x^{\prime}$ is kept fixed, $\sigma$ can be considered to be an ordinary scalar function of $x$. According to Equation (57), this function is a solution to the nonlinear differential equation $\frac{1}{2} g^{\alpha \beta} \sigma_{\alpha} \sigma_{\beta}=\sigma$. Suppose that we are presented with such a scalar field. What can we say about it?

An additional differentiation of the defining equation reveals that the vector $\sigma^{\alpha} \equiv \sigma^{; \alpha}$ satisfies

$$
\sigma_{; \beta}^{\alpha} \sigma^{\beta}=\sigma^{\alpha}
$$

which is the geodesic equation in a non-affine parameterization. The vector field is therefore tangent to a congruence of geodesics. The geodesics are timelike where $\sigma<0$, they are spacelike where $\sigma>0$, and they are null where $\sigma=0$. Here, for concreteness, we shall consider only the timelike subset of the congruence.

The vector

$$
u^{\alpha}=\frac{\sigma^{\alpha}}{|2 \sigma|^{1 / 2}}
$$

is a normalized tangent vector that satisfies the geodesic equation in affine-parameter form: $u_{; \beta}^{\alpha} u^{\beta}=$ 0 . The parameter $\lambda$ is then proper time $\tau$. If $\lambda^{*}$ denotes the original parameterization of the geodesics, we have that $d \lambda^{*} / d \tau=|2 \sigma|^{-1 / 2}$, and we see that the original parameterization is singular at $\sigma=0$.

In the affine parameterization, the expansion of the congruence is calculated to be

$$
\theta=\frac{\theta^{*}}{|2 \sigma|^{1 / 2}}, \quad \theta^{*}=\sigma_{; \alpha}^{\alpha}-1
$$

where $\theta^{*}=(\delta V)^{-1}\left(d / d \lambda^{*}\right)(\delta V)$ is the expansion in the original parameterization ( $\delta V$ is the congruence's cross-sectional volume). While $\theta^{*}$ is well behaved in the limit $\sigma \rightarrow 0$ (we shall see below

Living Reviews in Relativity

http://www. livingreviews.org//rr-2004-6 
that $\theta^{*} \rightarrow 3$ ), we have that $\theta \rightarrow \infty$. This means that the point $x^{\prime}$ at which $\sigma=0$ is a caustic of the congruence: All geodesics emanate from this point.

These considerations, which all follow from a postulated relation $\frac{1}{2} g^{\alpha \beta} \sigma_{\alpha} \sigma_{\beta}=\sigma$, are clearly compatible with our preceding explicit construction of the world function.

\subsection{Coincidence limits}

It is useful to determine the limiting behaviour of the bitensors $\sigma \ldots$ as $x$ approaches $x^{\prime}$. We introduce the notation

$$
\left[\Omega_{\ldots}\right]=\lim _{x \rightarrow x^{\prime}} \Omega_{\ldots}\left(x, x^{\prime}\right)=\text { a tensor at } x^{\prime}
$$

to designate the limit of any bitensor $\Omega_{\ldots}\left(x, x^{\prime}\right)$ as $x$ approaches $x^{\prime}$; this is called the coincidence limit of the bitensor. We assume that the coincidence limit is a unique tensorial function of the base point $x^{\prime}$, independent of the direction in which the limit is taken. In other words, if the limit is computed by letting $\lambda \rightarrow \lambda_{0}$ after evaluating $\Omega_{\ldots} .\left(z, x^{\prime}\right)$ as a function of $\lambda$ on a specified geodesic $\beta$, it is assumed that the answer does not depend on the choice of geodesic.

\subsubsection{Computation of coincidence limits}

From Equations $(53,55,56)$ we already have

$$
[\sigma]=0, \quad\left[\sigma_{\alpha}\right]=\left[\sigma_{\alpha^{\prime}}\right]=0 .
$$

Additional results are obtained by repeated differentiation of the relations (57) and (58). For example, Equation (57) implies $\sigma_{\gamma}=g^{\alpha \beta} \sigma_{\alpha} \sigma_{\beta \gamma}=\sigma^{\beta} \sigma_{\beta \gamma}$, or $\left(g_{\beta \gamma}-\sigma_{\beta \gamma}\right) t^{\beta}=0$ after using Equation (55). From the assumption stated in the preceding paragraph, $\sigma_{\beta \gamma}$ becomes independent of $t^{\beta}$ in the limit $x \rightarrow x^{\prime}$, and we arrive at $\left[\sigma_{\alpha \beta}\right]=g_{\alpha^{\prime} \beta^{\prime}}$. By very similar calculations we obtain all other coincidence limits for the second derivatives of the world function. The results are

$$
\left[\sigma_{\alpha \beta}\right]=\left[\sigma_{\alpha^{\prime} \beta^{\prime}}\right]=g_{\alpha^{\prime} \beta^{\prime}}, \quad\left[\sigma_{\alpha \beta^{\prime}}\right]=\left[\sigma_{\alpha^{\prime} \beta}\right]=-g_{\alpha^{\prime} \beta^{\prime}} .
$$

From these relations we infer that $\left[\sigma_{\alpha}^{\alpha}\right]=4$, so that $\left[\theta^{*}\right]=3$, where $\theta^{*}$ was defined in Equation (61).

To generate coincidence limits of bitensors involving primed indices, it is efficient to invoke Synge's rule,

$$
\left[\sigma_{\ldots \alpha^{\prime}}\right]=\left[\sigma_{\ldots}\right]_{; \alpha^{\prime}}-\left[\sigma_{\ldots \alpha}\right],
$$

in which "..." designates any combination of primed and unprimed indices; this rule will be established below. For example, according to Synge's rule we have $\left[\sigma_{\alpha \beta^{\prime}}\right]=\left[\sigma_{\alpha}\right]_{; \beta^{\prime}}-\left[\sigma_{\alpha \beta}\right]$, and since the coincidence limit of $\sigma_{\alpha}$ is zero, this gives us $\left[\sigma_{\alpha \beta^{\prime}}\right]=-\left[\sigma_{\alpha \beta}\right]=-g_{\alpha^{\prime} \beta^{\prime}}$, as was stated in Equation (63). Similarly, $\left[\sigma_{\alpha^{\prime} \beta^{\prime}}\right]=\left[\sigma_{\alpha^{\prime}}\right]_{; \beta^{\prime}}-\left[\sigma_{\alpha^{\prime} \beta}\right]=-\left[\sigma_{\beta \alpha^{\prime}}\right]=g_{\alpha^{\prime} \beta^{\prime}}$. The results of Equation (63) can thus all be generated from the known result for $\left[\sigma_{\alpha \beta}\right]$.

The coincidence limits of Equation (63) were derived from the relation $\sigma_{\alpha}=\sigma^{\delta}{ }_{\alpha} \sigma_{\delta}$. We now differentiate this twice more and obtain $\sigma_{\alpha \beta \gamma}=\sigma_{\alpha \beta \gamma}^{\delta} \sigma_{\delta}+\sigma_{\alpha \beta}^{\delta} \sigma_{\delta \gamma}+\sigma_{\alpha \gamma}^{\delta} \sigma_{\delta \beta}+\sigma^{\delta}{ }_{\alpha} \sigma_{\delta \beta \gamma}$. At coincidence we have

$$
\left[\sigma_{\alpha \beta \gamma}\right]=\left[\sigma_{\alpha \beta}^{\delta}\right] g_{\delta^{\prime} \gamma^{\prime}}+\left[\sigma_{\alpha \gamma}^{\delta}\right] g_{\delta^{\prime} \beta^{\prime}}+\delta_{\alpha^{\prime}}^{\delta^{\prime}}\left[\sigma_{\delta \beta \gamma}\right],
$$

or $\left[\sigma_{\gamma \alpha \beta}\right]+\left[\sigma_{\beta \alpha \gamma}\right]=0$ if we recognize that the operations of raising or lowering indices and taking the limit $x \rightarrow x^{\prime}$ commute. Noting the symmetries of $\sigma_{\alpha \beta}$, this gives us $\left[\sigma_{\alpha \gamma \beta}\right]+\left[\sigma_{\alpha \beta \gamma}\right]=0$, or $2\left[\sigma_{\alpha \beta \gamma}\right]-\left[R_{\alpha \beta \gamma}^{\delta} \sigma_{\delta}\right]=0$, or $2\left[\sigma_{\alpha \beta \gamma}\right]=R_{\alpha^{\prime} \beta^{\prime} \gamma^{\prime}}^{\delta^{\prime}}\left[\sigma_{\delta^{\prime}}\right]$. Since the last factor is zero, we arrive at

$$
\left[\sigma_{\alpha \beta \gamma}\right]=\left[\sigma_{\alpha \beta \gamma^{\prime}}\right]=\left[\sigma_{\alpha \beta^{\prime} \gamma^{\prime}}\right]=\left[\sigma_{\alpha^{\prime} \beta^{\prime} \gamma^{\prime}}\right]=0 .
$$

The last three results were derived from $\left[\sigma_{\alpha \beta \gamma}\right]=0$ by employing Synge's rule. 
We now differentiate the relation $\sigma_{\alpha}=\sigma_{\alpha}^{\delta} \sigma_{\delta}$ three times and obtain $\sigma_{\alpha \beta \gamma \delta}=\sigma_{\alpha \beta \gamma \delta}^{\epsilon} \sigma_{\epsilon}+\sigma_{\alpha \beta \gamma}^{\epsilon} \sigma_{\epsilon \delta}+\sigma_{\alpha \beta \delta}^{\epsilon} \sigma_{\epsilon \gamma}+\sigma_{\alpha \gamma \delta}^{\epsilon} \sigma_{\epsilon \beta}+\sigma_{\alpha \beta}^{\epsilon} \sigma_{\epsilon \gamma \delta}+\sigma_{\alpha \gamma}^{\epsilon} \sigma_{\epsilon \beta \delta}+\sigma_{\alpha \delta}^{\epsilon} \sigma_{\epsilon \beta \gamma}+\sigma_{\alpha}^{\epsilon} \sigma_{\epsilon \beta \gamma \delta}$.

At coincidence this reduces to $\left[\sigma_{\alpha \beta \gamma \delta}\right]+\left[\sigma_{\alpha \delta \beta \gamma}\right]+\left[\sigma_{\alpha \gamma \beta \delta}\right]=0$. To simplify the third term we differentiate Ricci's identity $\sigma_{\alpha \gamma \beta}=\sigma_{\alpha \beta \gamma}-R_{\alpha \beta \gamma}^{\epsilon} \sigma_{\epsilon}$ with respect to $x^{\delta}$ and then take the coincidence limit. This gives us $\left[\sigma_{\alpha \gamma \beta \delta}\right]=\left[\sigma_{\alpha \beta \gamma \delta}\right]+R_{\alpha^{\prime} \delta^{\prime} \beta^{\prime} \gamma^{\prime}}$. The same manipulations on the second term give $\left[\sigma_{\alpha \delta \beta \gamma}\right]=\left[\sigma_{\alpha \beta \delta \gamma}\right]+R_{\alpha^{\prime} \gamma^{\prime} \beta^{\prime} \delta^{\prime}}$. Using the identity $\sigma_{\alpha \beta \delta \gamma}=\sigma_{\alpha \beta \gamma \delta}-R_{\alpha \gamma \delta}^{\epsilon} \sigma_{\epsilon \beta}-R_{\beta \gamma \delta}^{\epsilon} \sigma_{\alpha \epsilon}$ and the symmetries of the Riemann tensor, it is then easy to show that $\left[\sigma_{\alpha \beta \delta \gamma}\right]=\left[\sigma_{\alpha \beta \gamma \delta}\right]$. Gathering the results, we obtain $3\left[\sigma_{\alpha \beta \gamma \delta}\right]+R_{\alpha^{\prime} \gamma^{\prime} \beta^{\prime} \delta^{\prime}}+R_{\alpha^{\prime} \delta^{\prime} \beta^{\prime} \gamma^{\prime}}=0$, and Synge's rule allows us to generalize this to any combination of primed and unprimed indices. Our final results are

$$
\begin{aligned}
{\left[\sigma_{\alpha \beta \gamma \delta}\right] } & =-\frac{1}{3}\left(R_{\alpha^{\prime} \gamma^{\prime} \beta^{\prime} \delta^{\prime}}+R_{\alpha^{\prime} \delta^{\prime} \beta^{\prime} \gamma^{\prime}}\right), \\
{\left[\sigma_{\alpha \beta \gamma \delta^{\prime}}\right] } & =\frac{1}{3}\left(R_{\alpha^{\prime} \gamma^{\prime} \beta^{\prime} \delta^{\prime}}+R_{\alpha^{\prime} \delta^{\prime} \beta^{\prime} \gamma^{\prime}}\right), \\
{\left[\sigma_{\alpha \beta \gamma^{\prime} \delta^{\prime}}\right] } & =-\frac{1}{3}\left(R_{\alpha^{\prime} \gamma^{\prime} \beta^{\prime} \delta^{\prime}}+R_{\alpha^{\prime} \delta^{\prime} \beta^{\prime} \gamma^{\prime}}\right), \\
{\left[\sigma_{\alpha \beta^{\prime} \gamma^{\prime} \delta^{\prime}}\right] } & =-\frac{1}{3}\left(R_{\alpha^{\prime} \beta^{\prime} \gamma^{\prime} \delta^{\prime}}+R_{\alpha^{\prime} \gamma^{\prime} \beta^{\prime} \delta^{\prime}}\right), \\
{\left[\sigma_{\alpha^{\prime} \beta^{\prime} \gamma^{\prime} \delta^{\prime}}\right] } & =-\frac{1}{3}\left(R_{\alpha^{\prime} \gamma^{\prime} \beta^{\prime} \delta^{\prime}}+R_{\alpha^{\prime} \delta^{\prime} \beta^{\prime} \gamma^{\prime}}\right) .
\end{aligned}
$$

\subsubsection{Derivation of Synge's rule}

We begin with any bitensor $\Omega_{A B^{\prime}}\left(x, x^{\prime}\right)$ in which $A=\alpha \ldots \beta$ is a multi-index that represents any number of unprimed indices, and $B^{\prime}=\gamma^{\prime} \ldots \delta^{\prime}$ a multi-index that represents any number of primed indices. (It does not matter whether the primed and unprimed indices are segregated or mixed.) On the geodesic $\beta$ that links $x$ to $x^{\prime}$ we introduce an ordinary tensor $P^{M}(z)$ where $M$ is a multiindex that contains the same number of indices as $A$. This tensor is arbitrary, but we assume that it is parallel transported on $\beta$; this means that it satisfies $P_{; \alpha}^{A} t^{\alpha}=0$ at $x$. Similarly, we introduce an ordinary tensor $Q^{N}(z)$ in which $N$ contains the same number of indices as $B^{\prime}$. This tensor is arbitrary, but we assume that it is parallel transported on $\beta$; at $x^{\prime}$ it satisfies $Q_{; \alpha^{\prime}}^{B^{\prime}} t^{\alpha^{\prime}}=0$. With $\Omega, P$, and $Q$ we form a biscalar $H\left(x, x^{\prime}\right)$ defined by

$$
H\left(x, x^{\prime}\right)=\Omega_{A B^{\prime}}\left(x, x^{\prime}\right) P^{A}(x) Q^{B^{\prime}}\left(x^{\prime}\right)
$$

Having specified the geodesic that links $x$ to $x^{\prime}$, we can consider $H$ to be a function of $\lambda_{0}$ and $\lambda_{1}$. If $\lambda_{1}$ is not much larger than $\lambda_{0}$ (so that $x$ is not far from $x^{\prime}$ ), we can express $H\left(\lambda_{1}, \lambda_{0}\right)$ as

$$
H\left(\lambda_{1}, \lambda_{0}\right)=H\left(\lambda_{0}, \lambda_{0}\right)+\left.\left(\lambda_{1}-\lambda_{0}\right) \frac{\partial H}{\partial \lambda_{1}}\right|_{\lambda_{1}=\lambda_{0}}+\ldots
$$

Alternatively,

$$
H\left(\lambda_{1}, \lambda_{0}\right)=H\left(\lambda_{1}, \lambda_{1}\right)-\left.\left(\lambda_{1}-\lambda_{0}\right) \frac{\partial H}{\partial \lambda_{0}}\right|_{\lambda_{0}=\lambda_{1}}+\ldots,
$$

and these two expressions give

$$
\frac{d}{d \lambda_{0}} H\left(\lambda_{0}, \lambda_{0}\right)=\left.\frac{\partial H}{\partial \lambda_{0}}\right|_{\lambda_{0}=\lambda_{1}}+\left.\frac{\partial H}{\partial \lambda_{1}}\right|_{\lambda_{1}=\lambda_{0}},
$$

Living Reviews in Relativity

http://www. livingreviews . org/Irr-2004-6 
because the left-hand side is the limit of $\left[H\left(\lambda_{1}, \lambda_{1}\right)-H\left(\lambda_{0}, \lambda_{0}\right)\right] /\left(\lambda_{1}-\lambda_{0}\right)$ when $\lambda_{1} \rightarrow \lambda_{0}$. The partial derivative of $H$ with respect to $\lambda_{0}$ is equal to $\Omega_{A B^{\prime} ; \alpha^{\prime}} t^{\alpha^{\prime}} P^{A} Q^{B^{\prime}}$, and in the limit this becomes $\left[\Omega_{A B^{\prime} ; \alpha^{\prime}}\right] t^{\alpha^{\prime}} P^{A^{\prime}} Q^{B^{\prime}}$. Similarly, the partial derivative of $H$ with respect to $\lambda_{1}$ is $\Omega_{A B^{\prime} ; \alpha} t^{\alpha} P^{A} Q^{B^{\prime}}$, and in the limit $\lambda_{1} \rightarrow \lambda_{0}$ this becomes $\left[\Omega_{A B^{\prime} ; \alpha}\right] t^{\alpha^{\prime}} P^{A^{\prime}} Q^{B^{\prime}}$. Finally, $H\left(\lambda_{0}, \lambda_{0}\right)=\left[\Omega_{A B^{\prime}}\right] P^{A^{\prime}} Q^{B^{\prime}}$, and its derivative with respect to $\lambda_{0}$ is $\left[\Omega_{A B^{\prime}}\right]_{; \alpha^{\prime}} t^{\alpha^{\prime}} P^{A^{\prime}} Q^{B^{\prime}}$. Gathering the results we find that

$$
\left\{\left[\Omega_{A B^{\prime}}\right]_{; \alpha^{\prime}}-\left[\Omega_{A B^{\prime} ; \alpha^{\prime}}\right]-\left[\Omega_{A B^{\prime} ; \alpha}\right]\right\} t^{\alpha^{\prime}} P^{A^{\prime}} Q^{B^{\prime}}=0,
$$

and the final statement of Synge's rule,

$$
\left[\Omega_{A B^{\prime}}\right]_{; \alpha^{\prime}}=\left[\Omega_{A B^{\prime} ; \alpha^{\prime}}\right]+\left[\Omega_{A B^{\prime} ; \alpha}\right],
$$

follows from the fact that the tensors $P^{M}$ and $Q^{N}$, and the direction of the selected geodesic $\beta$, are all arbitrary. Equation (67) reduces to Equation (64) when $\sigma \ldots$ is substituted in place of $\Omega_{A B^{\prime}}$.

\subsection{Parallel propagator}

\subsubsection{Tetrad on $\beta$}

On the geodesic $\beta$ that links $x$ to $x^{\prime}$ we introduce an orthonormal basis $e_{\mathrm{a}}^{\mu}(z)$ that is parallel transported on the geodesic. The frame indices ${ }^{3} \mathrm{a}, \mathrm{b}, \ldots$, run from 0 to 3 , and the frame vectors satisfy

$$
g_{\mu \nu} e_{\mathrm{a}}^{\mu} e_{\mathrm{b}}^{\nu}=\eta_{\mathrm{ab}}, \quad \frac{D e_{\mathrm{a}}^{\mu}}{d \lambda}=0,
$$

where $\eta_{\mathrm{ab}}=\operatorname{diag}(-1,1,1,1)$ is the Minkowski metric (which we shall use to raise and lower frame indices). We have the completeness relations

$$
g^{\mu \nu}=\eta^{\mathrm{ab}} e_{\mathrm{a}}^{\mu} e_{\mathrm{b}}^{\nu}
$$

and we define a dual tetrad $e_{\mu}^{\mathrm{a}}(z)$ by

$$
e_{\mu}^{\mathrm{a}} \equiv \eta^{\mathrm{ab}} g_{\mu \nu} e_{\mathrm{b}}^{\nu}
$$

this is also parallel transported on $\beta$. In terms of the dual tetrad the completeness relations take the form

$$
g_{\mu \nu}=\eta_{\mathrm{ab}} e_{\mu}^{\mathrm{a}} e_{\nu}^{\mathrm{b}}
$$

and it is easy to show that the tetrad and its dual satisfy $e_{\mu}^{\mathrm{a}} e_{\mathrm{b}}^{\mu}=\delta_{\mathrm{b}}^{\mathrm{a}}$ and $e_{\nu}^{\mathrm{a}} e_{\mathrm{a}}^{\mu}=\delta^{\mu}{ }_{\nu}$. Equations (68, $69,70,71$ ) hold everywhere on $\beta$. In particular, with an appropriate change of notation they hold at $x^{\prime}$ and $x$; for example, $g_{\alpha \beta}=\eta_{\mathrm{ab}} e_{\alpha}^{\mathrm{a}} e_{\beta}^{\mathrm{b}}$ is the metric at $x$.

\subsubsection{Definition and properties of the parallel propagator}

Any vector field $A^{\mu}(z)$ on $\beta$ can be decomposed in the basis $e_{\mathrm{a}}^{\mu}: A^{\mu}=A^{\mathrm{a}} e_{\mathrm{a}}^{\mu}$, and the vector's frame components are given by $A^{\mathrm{a}}=A^{\mu} e_{\mu}^{\mathrm{a}}$. If $A^{\mu}$ is parallel transported on the geodesic, then the coefficients $A^{\mathrm{a}}$ are constants. The vector at $x$ can then be expressed as $A^{\alpha}=\left(A^{\alpha^{\prime}} e_{\alpha^{\prime}}^{\mathrm{a}}\right) e_{\mathrm{a}}^{\alpha}$, or

$$
A^{\alpha}(x)=g_{\alpha^{\prime}}^{\alpha}\left(x, x^{\prime}\right) A^{\alpha^{\prime}}\left(x^{\prime}\right), \quad g_{\alpha^{\prime}}^{\alpha}\left(x, x^{\prime}\right) \equiv e_{\mathrm{a}}^{\alpha}(x) e_{\alpha^{\prime}}^{\mathrm{a}}\left(x^{\prime}\right) .
$$

\footnotetext{
${ }^{3}$ Note that I use sans-serif symbols for the frame indices. This is to distinguish them from another set of frame indices that will appear below. The frame indices introduced here run from 0 to 3 ; those to be introduced later will run from 1 to 3 .
} 
The object $g_{\alpha^{\prime}}^{\alpha}=e_{\mathrm{a}}^{\alpha} e_{\alpha^{\prime}}^{\mathrm{a}}$ is the parallel propagator: It takes a vector at $x^{\prime}$ and parallel-transports it to $x$ along the unique geodesic that links these points.

Similarly, we find that

$$
A^{\alpha^{\prime}}\left(x^{\prime}\right)=g_{\alpha}^{\alpha^{\prime}}\left(x^{\prime}, x\right) A^{\alpha}(x), \quad g_{\alpha}^{\alpha^{\prime}}\left(x^{\prime}, x\right) \equiv e_{\mathrm{a}}^{\alpha^{\prime}}\left(x^{\prime}\right) e_{\alpha}^{\mathrm{a}}(x),
$$

and we see that $g_{\alpha}^{\alpha^{\prime}}=e_{\mathrm{a}}^{\alpha^{\prime}} e_{\alpha}^{\mathrm{a}}$ performs the inverse operation: It takes a vector at $x$ and paralleltransports it back to $x^{\prime}$. Clearly,

$$
g_{\alpha^{\prime}}^{\alpha} g_{\beta}^{\alpha^{\prime}}=\delta_{\beta}^{\alpha}, \quad g_{\alpha}^{\alpha^{\prime}} g_{\beta^{\prime}}^{\alpha}=\delta_{\beta^{\prime}}^{\alpha^{\prime}},
$$

and these relations formally express the fact that $g_{\alpha}^{\alpha^{\prime}}$ is the inverse of $g_{\alpha^{\prime}}^{\alpha}$.

The relation $g_{\alpha^{\prime}}^{\alpha}=e_{\mathrm{a}}^{\alpha} e_{\alpha^{\prime}}^{\mathrm{a}}$ can also be expressed as $g_{\alpha}{ }^{\alpha^{\prime}}=e_{\alpha}^{\mathrm{a}} e_{\mathrm{a}}^{\alpha^{\prime}}$, and this reveals that

$$
g_{\alpha}^{\alpha^{\prime}}\left(x, x^{\prime}\right)=g_{\alpha}^{\alpha^{\prime}}\left(x^{\prime}, x\right), \quad g_{\alpha^{\prime}}^{\alpha}\left(x^{\prime}, x\right)=g_{\alpha^{\prime}}^{\alpha}\left(x, x^{\prime}\right) .
$$

The ordering of the indices, and the ordering of the arguments, are therefore arbitrary.

The action of the parallel propagator on tensors of arbitrary ranks is easy to figure out. For example, suppose that the dual vector $p_{\mu}=p_{a} e_{\mu}^{a}$ is parallel transported on $\beta$. Then the frame components $p_{\mathrm{a}}=p_{\mu} e_{\mathrm{a}}^{\mu}$ are constants, and the dual vector at $x$ can be expressed as $p_{\alpha}=\left(p_{\alpha^{\prime}} e_{\mathrm{a}}^{\alpha^{\prime}}\right) e_{\mathrm{a}}^{\alpha}$, or

$$
p_{\alpha}(x)=g_{\alpha}^{\alpha^{\prime}}\left(x^{\prime}, x\right) p_{\alpha^{\prime}}\left(x^{\prime}\right) .
$$

It is therefore the inverse propagator $g_{\alpha}^{\alpha^{\prime}}$ that takes a dual vector at $x^{\prime}$ and parallel-transports it to $x$. As another example, it is easy to show that a tensor $A^{\alpha \beta}$ at $x$ obtained by parallel transport from $x^{\prime}$ must be given by

$$
A^{\alpha \beta}(x)=g_{\alpha^{\prime}}^{\alpha}\left(x, x^{\prime}\right) g_{\beta^{\prime}}^{\beta}\left(x, x^{\prime}\right) A^{\alpha^{\prime} \beta^{\prime}}\left(x^{\prime}\right) .
$$

Here we need two occurrences of the parallel propagator, one for each tensorial index. Because the metric tensor is covariantly constant, it is automatically parallel transported on $\beta$, and a special case of Equation (77) is therefore $g_{\alpha \beta}=g_{\alpha}^{\alpha^{\prime}} g_{\beta}^{\beta^{\prime}} g_{\alpha^{\prime} \beta^{\prime}}$.

Because the basis vectors are parallel transported on $\beta$, they satisfy $e_{\mathrm{a} ; \beta}^{\alpha} \sigma^{\beta}=0$ at $x$ and $e_{\mathrm{a} ; \beta^{\prime}}^{\alpha^{\prime}} \sigma^{\beta^{\prime}}=0$ at $x^{\prime}$. This immediately implies that the parallel propagators must satisfy

$$
g_{\alpha^{\prime} ; \beta}^{\alpha} \sigma^{\beta}=g_{\alpha^{\prime} ; \beta^{\prime}}^{\alpha} \sigma^{\beta^{\prime}}=0, \quad g_{\alpha ; \beta}^{\alpha^{\prime}} \sigma^{\beta}=g_{\alpha ; \beta^{\prime}}^{\alpha^{\prime}} \sigma^{\beta^{\prime}}=0 .
$$

Another useful property of the parallel propagator follows from the fact that if $t^{\mu}=d z^{\mu} / d \lambda$ is tangent to the geodesic connecting $x$ to $x^{\prime}$, then $t^{\alpha}=g_{\alpha^{\prime}}^{\alpha} t^{\alpha^{\prime}}$. Using Equations (55) and (56), this observation gives us the relations

$$
\sigma_{\alpha}=-g_{\alpha}^{\alpha^{\prime}} \sigma_{\alpha^{\prime}}, \quad \sigma_{\alpha^{\prime}}=-g_{\alpha^{\prime}}^{\alpha} \sigma_{\alpha}
$$

\subsubsection{Coincidence limits}

Equation (72) and the completeness relations of Equations (69) or (71) imply that

$$
\left[g_{\beta^{\prime}}^{\alpha}\right]=\delta_{\beta^{\prime}}^{\alpha^{\prime}} .
$$

Other coincidence limits are obtained by differentiation of Equations (78). For example, the relation $g_{\beta^{\prime} ; \gamma}^{\alpha} \sigma^{\gamma}=0$ implies $g_{\beta^{\prime} ; \gamma \delta}^{\alpha} \sigma^{\gamma}+g_{\beta^{\prime} ; \gamma}^{\alpha} \sigma_{\delta}^{\gamma}=0$, and at coincidence we have

$$
\left[g_{\beta^{\prime} ; \gamma}^{\alpha}\right]=\left[g_{\beta^{\prime} ; \gamma^{\prime}}^{\alpha}\right]=0
$$

Living Reviews in Relativity

http://www. livingreviews.org/lrr-2004-6 
the second result was obtained by applying Synge's rule on the first result. Further differentiation gives

$$
g_{\beta^{\prime} ; \gamma \delta \epsilon}^{\alpha} \sigma^{\gamma}+g_{\beta^{\prime} ; \gamma \delta}^{\alpha} \sigma_{\epsilon}^{\gamma}+g_{\beta^{\prime} ; \gamma \epsilon}^{\alpha} \sigma_{\delta}^{\gamma}+g_{\beta^{\prime} ; \gamma}^{\alpha} \sigma_{\delta \epsilon}^{\gamma}=0,
$$

and at coincidence we have $\left[g_{\beta^{\prime} ; \gamma \delta}^{\alpha}\right]+\left[g_{\beta^{\prime} ; \delta \gamma}^{\alpha}\right]=0$, or $2\left[g_{\beta^{\prime} ; \gamma \delta}^{\alpha}\right]+R_{\beta^{\prime} \gamma^{\prime} \delta^{\prime}}^{\alpha^{\prime}}=0$. The coincidence limit for $g_{\beta^{\prime} ; \gamma \delta^{\prime}}^{\alpha}=g_{\beta^{\prime} ; \delta^{\prime} \gamma}^{\alpha}$ can then be obtained from Synge's rule, and an additional application of the rule gives $\left[g_{\beta^{\prime} ; \gamma^{\prime} \delta^{\prime}}^{\alpha}\right.$. Our results are

$$
\begin{aligned}
{\left[g_{\beta^{\prime} ; \gamma \delta}^{\alpha}\right] } & =-\frac{1}{2} R_{\beta^{\prime} \gamma^{\prime} \delta^{\prime}}^{\alpha^{\prime}}, & {\left[g_{\beta^{\prime} ; \gamma \delta^{\prime}}^{\alpha}\right.} & =\frac{1}{2} R_{\beta^{\prime} \gamma^{\prime} \delta^{\prime}}^{\alpha^{\prime}}, \\
{\left[g_{\beta^{\prime} ; \gamma^{\prime} \delta}^{\alpha}\right] } & =-\frac{1}{2} R_{\beta^{\prime} \gamma^{\prime} \delta^{\prime}}^{\alpha^{\prime}}, & {\left[g_{\beta^{\prime} ; \gamma^{\prime} \delta^{\prime}}^{\alpha}\right] } & =\frac{1}{2} R_{\beta^{\prime} \gamma^{\prime} \delta^{\prime}}^{\alpha^{\prime}}
\end{aligned}
$$

\subsection{Expansion of bitensors near coincidence}

\subsubsection{General method}

We would like to express a bitensor $\Omega_{\alpha^{\prime} \beta^{\prime}}\left(x, x^{\prime}\right)$ near coincidence as an expansion in powers of $-\sigma^{\alpha^{\prime}}\left(x, x^{\prime}\right)$, the closest analogue in curved spacetime to the flat-spacetime quantity $\left(x-x^{\prime}\right)^{\alpha}$. For concreteness we shall consider the case of rank-2 bitensor, and for the moment we will assume that the bitensor's indices all refer to the base point $x^{\prime}$.

The expansion we seek is of the form

$$
\Omega_{\alpha^{\prime} \beta^{\prime}}\left(x, x^{\prime}\right)=A_{\alpha^{\prime} \beta^{\prime}}+A_{\alpha^{\prime} \beta^{\prime} \gamma^{\prime}} \sigma^{\gamma^{\prime}}+\frac{1}{2} A_{\alpha^{\prime} \beta^{\prime} \gamma^{\prime} \delta^{\prime}} \sigma^{\gamma^{\prime}} \sigma^{\delta^{\prime}}+\mathcal{O}\left(\epsilon^{3}\right),
$$

in which the "expansion coefficients" $A_{\alpha^{\prime} \beta^{\prime}}, A_{\alpha^{\prime} \beta^{\prime} \gamma^{\prime}}$, and $A_{\alpha^{\prime} \beta^{\prime} \gamma^{\prime} \delta^{\prime}}$ are all ordinary tensors at $x^{\prime}$; this last tensor is symmetric in the pair of indices $\gamma^{\prime}$ and $\delta^{\prime}$, and $\epsilon$ measures the size of a typical component of $\sigma^{\alpha^{\prime}}$.

To find the expansion coefficients we differentiate Equation (83) repeatedly and take coincidence limits. Equation (83) immediately implies $\left[\Omega_{\alpha^{\prime} \beta^{\prime}}\right]=A_{\alpha^{\prime} \beta^{\prime}}$. After one differentiation we obtain $\Omega_{\alpha^{\prime} \beta^{\prime} ; \gamma^{\prime}}=A_{\alpha^{\prime} \beta^{\prime} ; \gamma^{\prime}}+A_{\alpha^{\prime} \beta^{\prime} \epsilon^{\prime} ; \gamma^{\prime}} \sigma^{\epsilon^{\prime}}+A_{\alpha^{\prime} \beta^{\prime} \epsilon^{\prime}} \sigma_{\gamma^{\prime}}^{\epsilon^{\prime}}+\frac{1}{2} A_{\alpha^{\prime} \beta^{\prime} \epsilon^{\prime} \iota^{\prime} ; \gamma^{\prime}} \sigma^{\epsilon^{\prime}} \sigma^{\iota^{\prime}}+A_{\alpha^{\prime} \beta^{\prime} \epsilon^{\prime} \iota^{\prime}} \sigma^{\epsilon^{\prime}} \sigma^{\iota^{\prime}} \gamma^{\prime}+\mathcal{O}\left(\epsilon^{2}\right)$, and at coincidence this reduces to $\left[\Omega_{\alpha^{\prime} \beta^{\prime} ; \gamma^{\prime}}\right]=A_{\alpha^{\prime} \beta^{\prime} ; \gamma^{\prime}}+A_{\alpha^{\prime} \beta^{\prime} \gamma^{\prime}}$. Taking the coincidence limit after two differentiations yields $\left[\Omega_{\alpha^{\prime} \beta^{\prime} ; \gamma^{\prime} \delta^{\prime}}\right]=A_{\alpha^{\prime} \beta^{\prime} ; \gamma^{\prime} \delta^{\prime}}+A_{\alpha^{\prime} \beta^{\prime} \gamma^{\prime} ; \delta^{\prime}}+A_{\alpha^{\prime} \beta^{\prime} \delta^{\prime} ; \gamma^{\prime}}+A_{\alpha^{\prime} \beta^{\prime} \gamma^{\prime} \delta^{\prime}}$. The expansion coefficients are therefore

$$
\begin{aligned}
A_{\alpha^{\prime} \beta^{\prime}} & =\left[\Omega_{\alpha^{\prime} \beta^{\prime}}\right], \\
A_{\alpha^{\prime} \beta^{\prime} \gamma^{\prime}} & =\left[\Omega_{\alpha^{\prime} \beta^{\prime} ; \gamma^{\prime}}\right]-A_{\alpha^{\prime} \beta^{\prime} ; \gamma^{\prime}}, \\
A_{\alpha^{\prime} \beta^{\prime} \gamma^{\prime} \delta^{\prime}} & =\left[\Omega_{\alpha^{\prime} \beta^{\prime} ; \gamma^{\prime} \delta^{\prime}}\right]-A_{\alpha^{\prime} \beta^{\prime} ; \gamma^{\prime} \delta^{\prime}}-A_{\alpha^{\prime} \beta^{\prime} \gamma^{\prime} ; \delta^{\prime}}-A_{\alpha^{\prime} \beta^{\prime} \delta^{\prime} ; \gamma^{\prime}} .
\end{aligned}
$$

These results are to be substituted into Equation (83), and this gives us $\Omega_{\alpha^{\prime} \beta^{\prime}}\left(x, x^{\prime}\right)$ to second order in $\epsilon$.

Suppose now that the bitensor is $\Omega_{\alpha^{\prime} \beta}$, with one index referring to $x^{\prime}$ and the other to $x$. The previous procedure can be applied directly if we introduce an auxiliary bitensor $\tilde{\Omega}_{\alpha^{\prime} \beta^{\prime}} \equiv g_{\beta^{\prime}}{ } \Omega_{\alpha^{\prime} \beta}$ whose indices all refer to the point $x^{\prime}$. Then $\tilde{\Omega}_{\alpha^{\prime} \beta^{\prime}}$ can be expanded as in Equation (83), and the original bitensor is reconstructed as $\Omega_{\alpha^{\prime} \beta}=g^{\beta^{\prime}} \tilde{\Omega}_{\alpha^{\prime} \beta^{\prime}}$, or

$$
\Omega_{\alpha^{\prime} \beta}\left(x, x^{\prime}\right)=g_{\beta}^{\beta^{\prime}}\left(B_{\alpha^{\prime} \beta^{\prime}}+B_{\alpha^{\prime} \beta^{\prime} \gamma^{\prime}} \sigma^{\gamma^{\prime}}+\frac{1}{2} B_{\alpha^{\prime} \beta^{\prime} \gamma^{\prime} \delta^{\prime}} \sigma^{\gamma^{\prime}} \sigma^{\delta^{\prime}}\right)+\mathcal{O}\left(\epsilon^{3}\right) .
$$

The expansion coefficients can be obtained from the coincidence limits of $\tilde{\Omega}_{\alpha^{\prime} \beta^{\prime}}$ and its derivatives. It is convenient, however, to express them directly in terms of the original bitensor $\Omega_{\alpha^{\prime} \beta}$ by substituting the relation $\tilde{\Omega}_{\alpha^{\prime} \beta^{\prime}}=g_{\beta^{\prime}}{ }^{\prime} \Omega_{\alpha^{\prime} \beta}$ and its derivatives. After using the results of Equation (80, 
81, 82) we find

$$
\begin{aligned}
B_{\alpha^{\prime} \beta^{\prime}} & =\left[\Omega_{\alpha^{\prime} \beta}\right] \\
B_{\alpha^{\prime} \beta^{\prime} \gamma^{\prime}} & =\left[\Omega_{\alpha^{\prime} \beta ; \gamma^{\prime}}\right]-B_{\alpha^{\prime} \beta^{\prime} ; \gamma^{\prime}}, \\
B_{\alpha^{\prime} \beta^{\prime} \gamma^{\prime} \delta^{\prime}} & =\left[\Omega_{\alpha^{\prime} \beta ; \gamma^{\prime} \delta^{\prime}}\right]+\frac{1}{2} B_{\alpha^{\prime} \epsilon^{\prime}} R_{\beta^{\prime} \gamma^{\prime} \delta^{\prime}}^{\prime}-B_{\alpha^{\prime} \beta^{\prime} ; \gamma^{\prime} \delta^{\prime}}-B_{\alpha^{\prime} \beta^{\prime} \gamma^{\prime} ; \delta^{\prime}}-B_{\alpha^{\prime} \beta^{\prime} \delta^{\prime} ; \gamma^{\prime}} .
\end{aligned}
$$

The only difference with respect to Equation (85) is the presence of a Riemann-tensor term in $B_{\alpha^{\prime} \beta^{\prime} \gamma^{\prime} \delta^{\prime}}$.

Suppose finally that the bitensor to be expanded is $\Omega_{\alpha \beta}$, whose indices all refer to $x$. Much as we did before, we introduce an auxiliary bitensor $\tilde{\Omega}_{\alpha^{\prime} \beta^{\prime}}=g_{\alpha^{\prime}}^{\alpha} g_{\beta^{\prime}} \Omega_{\alpha \beta}$ whose indices all refer to $x^{\prime}$, we expand $\tilde{\Omega}_{\alpha^{\prime} \beta^{\prime}}$ as in Equation (83), and we then reconstruct the original bitensor. This gives us

$$
\Omega_{\alpha \beta}\left(x, x^{\prime}\right)=g_{\alpha}^{\alpha^{\prime}} g_{\beta}^{\beta^{\prime}}\left(C_{\alpha^{\prime} \beta^{\prime}}+C_{\alpha^{\prime} \beta^{\prime} \gamma^{\prime}} \sigma^{\gamma^{\prime}}+\frac{1}{2} C_{\alpha^{\prime} \beta^{\prime} \gamma^{\prime} \delta^{\prime}} \sigma^{\gamma^{\prime}} \sigma^{\delta^{\prime}}\right)+\mathcal{O}\left(\epsilon^{3}\right)
$$

and the expansion coefficients are now

$$
\begin{aligned}
C_{\alpha^{\prime} \beta^{\prime}} & =\left[\Omega_{\alpha \beta}\right], \\
C_{\alpha^{\prime} \beta^{\prime} \gamma^{\prime}} & =\left[\Omega_{\alpha \beta ; \gamma^{\prime}}\right]-C_{\alpha^{\prime} \beta^{\prime} ; \gamma^{\prime}}, \\
C_{\alpha^{\prime} \beta^{\prime} \gamma^{\prime} \delta^{\prime}} & =\left[\Omega_{\alpha \beta ; \gamma^{\prime} \delta^{\prime}}\right]+\frac{1}{2} C_{\alpha^{\prime} \epsilon^{\prime}} R_{\beta^{\prime} \gamma^{\prime} \delta^{\prime}}^{\epsilon^{\prime}}+\frac{1}{2} C_{\epsilon^{\prime} \beta^{\prime}} R_{\alpha^{\prime} \gamma^{\prime} \delta^{\prime}}^{\epsilon^{\prime}}-C_{\alpha^{\prime} \beta^{\prime} ; \gamma^{\prime} \delta^{\prime}}-C_{\alpha^{\prime} \beta^{\prime} \gamma^{\prime} ; \delta^{\prime}}-C_{\alpha^{\prime} \beta^{\prime} \delta^{\prime} ; \gamma^{\prime}} .
\end{aligned}
$$

This differs from Equation (86) by the presence of an additional Riemann-tensor term in $C_{\alpha^{\prime} \beta^{\prime} \gamma^{\prime} \delta^{\prime}}$.

\subsubsection{Special cases}

We now apply the general expansion method developed in the preceding Section 2.4.1 to the bitensors $\sigma_{\alpha^{\prime} \beta^{\prime}}, \sigma_{\alpha^{\prime} \beta}$, and $\sigma_{\alpha \beta}$. In the first instance we have $A_{\alpha^{\prime} \beta^{\prime}}=g_{\alpha^{\prime} \beta^{\prime}}, A_{\alpha^{\prime} \beta^{\prime} \gamma^{\prime}}=0$, and $A_{\alpha^{\prime} \beta^{\prime} \gamma^{\prime} \delta^{\prime}}=-\frac{1}{3}\left(R_{\alpha^{\prime} \gamma^{\prime} \beta^{\prime} \delta^{\prime}}+R_{\alpha^{\prime} \delta^{\prime} \beta^{\prime} \gamma^{\prime}}\right)$. In the second instance we have $B_{\alpha^{\prime} \beta^{\prime}}=-g_{\alpha^{\prime} \beta^{\prime}}, B_{\alpha^{\prime} \beta^{\prime} \gamma^{\prime}}=0$, and $B_{\alpha^{\prime} \beta^{\prime} \gamma^{\prime} \delta^{\prime}}=-\frac{1}{3}\left(R_{\beta^{\prime} \alpha^{\prime} \gamma^{\prime} \delta^{\prime}}+R_{\beta^{\prime} \gamma^{\prime} \alpha^{\prime} \delta^{\prime}}\right)-\frac{1}{2} R_{\alpha^{\prime} \beta^{\prime} \gamma^{\prime} \delta^{\prime}}=-\frac{1}{3} R_{\alpha^{\prime} \delta^{\prime} \beta^{\prime} \gamma^{\prime}}-\frac{1}{6} R_{\alpha^{\prime} \beta^{\prime} \gamma^{\prime} \delta^{\prime}}$. In the third instance we have $C_{\alpha^{\prime} \beta^{\prime}}=g_{\alpha^{\prime} \beta^{\prime}}, C_{\alpha^{\prime} \beta^{\prime} \gamma^{\prime}}=0$, and $C_{\alpha^{\prime} \beta^{\prime} \gamma^{\prime} \delta^{\prime}}=-\frac{1}{3}\left(R_{\alpha^{\prime} \gamma^{\prime} \beta^{\prime} \delta^{\prime}}+R_{\alpha^{\prime} \delta^{\prime} \beta^{\prime} \gamma^{\prime}}\right)$. This gives us the expansions

$$
\begin{aligned}
\sigma_{\alpha^{\prime} \beta^{\prime}} & =g_{\alpha^{\prime} \beta^{\prime}}-\frac{1}{3} R_{\alpha^{\prime} \gamma^{\prime} \beta^{\prime} \delta^{\prime}} \sigma^{\gamma^{\prime}} \sigma^{\delta^{\prime}}+\mathcal{O}\left(\epsilon^{3}\right) \\
\sigma_{\alpha^{\prime} \beta} & =-g_{\beta}^{\beta^{\prime}}\left(g_{\alpha^{\prime} \beta^{\prime}}+\frac{1}{6} R_{\alpha^{\prime} \gamma^{\prime} \beta^{\prime} \delta^{\prime}} \sigma^{\gamma^{\prime}} \sigma^{\delta^{\prime}}\right)+\mathcal{O}\left(\epsilon^{3}\right), \\
\sigma_{\alpha \beta} & =g_{\alpha}^{\alpha^{\prime}} g_{\beta^{\prime}}^{\beta^{\prime}}\left(g_{\alpha^{\prime} \beta^{\prime}}-\frac{1}{3} R_{\alpha^{\prime} \gamma^{\prime} \beta^{\prime} \delta^{\prime}} \sigma^{\gamma^{\prime}} \sigma^{\delta^{\prime}}\right)+\mathcal{O}\left(\epsilon^{3}\right) .
\end{aligned}
$$

Taking the trace of the last equation returns $\sigma_{\alpha}^{\alpha}=4-\frac{1}{3} R_{\gamma^{\prime} \delta^{\prime}} \sigma^{\gamma^{\prime}} \sigma^{\delta^{\prime}}+\mathcal{O}\left(\epsilon^{3}\right)$, or

$$
\theta^{*}=3-\frac{1}{3} R_{\alpha^{\prime} \beta^{\prime}} \sigma^{\alpha^{\prime}} \sigma^{\beta^{\prime}}+\mathcal{O}\left(\epsilon^{3}\right)
$$

where $\theta^{*} \equiv \sigma_{\alpha}^{\alpha}-1$ was shown in Section 2.1.4 to describe the expansion of the congruence of geodesics that emanate from $x^{\prime}$. Equation (91) reveals that timelike geodesics are focused if the Ricci tensor is nonzero and the strong energy condition holds: When $R_{\alpha^{\prime} \beta^{\prime}} \sigma^{\alpha^{\prime}} \sigma^{\beta^{\prime}}>0$ we see that $\theta^{*}$ is smaller than 3 , the value it would take in flat spacetime.

Living Reviews in Relativity

http: //www . livingreviews . org/lrr-2004-6 
The expansion method can easily be extended to bitensors of other tensorial ranks. In particular, it can be adapted to give expansions of the first derivatives of the parallel propagator. The expansions

$$
g_{\beta^{\prime} ; \gamma^{\prime}}^{\alpha}=\frac{1}{2} g_{\alpha^{\prime}}^{\alpha} R_{\beta^{\prime} \gamma^{\prime} \delta^{\prime}}^{\alpha^{\prime}} \sigma^{\delta^{\prime}}+\mathcal{O}\left(\epsilon^{2}\right), \quad g_{\beta^{\prime} ; \gamma}^{\alpha}=\frac{1}{2} g_{\alpha^{\prime}}^{\alpha} g_{\gamma}^{\gamma^{\prime}} R_{\beta^{\prime} \gamma^{\prime} \delta^{\prime}}^{\alpha^{\prime}} \sigma^{\delta^{\prime}}+\mathcal{O}\left(\epsilon^{2}\right)
$$

and thus easy to establish, and they will be needed in Section 4 of this review.

\subsubsection{Expansion of tensors}

The expansion method can also be applied to ordinary tensor fields. For concreteness, suppose that we wish to express a rank-2 tensor $A_{\alpha \beta}$ at a point $x$ in terms of its values (and that of its covariant derivatives) at a neighbouring point $x^{\prime}$. The tensor can be written as an expansion in powers of $-\sigma^{\alpha^{\prime}}\left(x, x^{\prime}\right)$, and in this case we have

$$
A_{\alpha \beta}(x)=g_{\alpha}^{\alpha^{\prime}} g_{\beta}^{\beta^{\prime}}\left(A_{\alpha^{\prime} \beta^{\prime}}-A_{\alpha^{\prime} \beta^{\prime} ; \gamma^{\prime}} \sigma^{\gamma^{\prime}}+\frac{1}{2} A_{\alpha^{\prime} \beta^{\prime} ; \gamma^{\prime} \delta^{\prime}} \sigma^{\gamma^{\prime}} \sigma^{\delta^{\prime}}\right)+\mathcal{O}\left(\epsilon^{3}\right) .
$$

If the tensor field is parallel transported on the geodesic $\beta$ that links $x$ to $x^{\prime}$, then Equation (93) reduces to Equation (77). The extension of this formula to tensors of other ranks is obvious.

To derive this result we express $A_{\mu \nu}(z)$, the restriction of the tensor field on $\beta$, in terms of its tetrad components $A_{\mathrm{ab}}(\lambda)=A_{\mu \nu} e_{\mathrm{a}}^{\mu} e_{\mathrm{b}}^{\nu}$. Recall from Section 2.3.1 that $e_{\mathrm{a}}^{\mu}$ is an orthonormal basis that is parallel transported on $\beta$; recall also that the affine parameter $\lambda$ ranges from $\lambda_{0}$ (its value at $x^{\prime}$ ) to $\lambda_{1}$ (its value at $x$ ). We have $A_{\alpha^{\prime} \beta^{\prime}}\left(x^{\prime}\right)=A_{\mathrm{ab}}\left(\lambda_{0}\right) e_{\alpha^{\prime}}^{\mathrm{a}} e_{\beta^{\prime}}^{\mathrm{b}}, A_{\alpha \beta}(x)=A_{\mathrm{ab}}\left(\lambda_{1}\right) e_{\alpha}^{\mathrm{a}} e_{\beta}^{\mathrm{b}}$, and $A_{\mathrm{ab}}\left(\lambda_{1}\right)$ can be expressed in terms of quantities at $\lambda=\lambda_{0}$ by straightforward Taylor expansion. Since, for example,

$$
\left.\left(\lambda_{1}-\lambda_{0}\right) \frac{d A_{\mathrm{ab}}}{d \lambda}\right|_{\lambda_{0}}=\left.\left(\lambda_{1}-\lambda_{0}\right)\left(A_{\mu \nu} e_{\mathrm{a}}^{\mu} e_{\mathrm{b}}^{\nu}\right)_{; \lambda} t^{\lambda}\right|_{\lambda_{0}}=\left.\left(\lambda_{1}-\lambda_{0}\right) A_{\mu \nu ; \lambda} e_{\mathrm{a}}^{\mu} e_{\mathrm{b}}^{\nu} t^{\lambda}\right|_{\lambda_{0}}=-A_{\alpha^{\prime} \beta^{\prime} ; \gamma^{\prime}} e_{\mathrm{a}}^{\alpha^{\prime}} e_{\mathrm{b}}^{\beta^{\prime}} \sigma^{\gamma^{\prime}},
$$

where we have used Equation (56), we arrive at Equation (93) after involving Equation (73).

\subsection{Van Vleck determinant}

\subsubsection{Definition and properties}

The van Vleck biscalar $\Delta\left(x, x^{\prime}\right)$ is defined by

$$
\Delta\left(x, x^{\prime}\right) \equiv \operatorname{det}\left[\Delta_{\beta^{\prime}}^{\alpha^{\prime}}\left(x, x^{\prime}\right)\right], \quad \Delta_{\beta^{\prime}}^{\alpha^{\prime}}\left(x, x^{\prime}\right) \equiv-g_{\alpha}^{\alpha^{\prime}}\left(x^{\prime}, x\right) \sigma_{\beta^{\prime}}^{\alpha}\left(x, x^{\prime}\right) .
$$

As we shall show below, it can also be expressed as

$$
\Delta\left(x, x^{\prime}\right)=-\frac{\operatorname{det}\left[-\sigma_{\alpha \beta^{\prime}}\left(x, x^{\prime}\right)\right]}{\sqrt{-g} \sqrt{-g^{\prime}}},
$$

where $g$ is the metric determinant at $x$ and $g^{\prime}$ the metric determinant at $x^{\prime}$.

Equations (63) and (80) imply that at coincidence, $\left[\Delta_{\beta^{\prime}}^{\alpha^{\prime}}\right]=\delta_{\beta^{\prime}}^{\alpha^{\prime}}$ and $[\Delta]=1$. Equation (89), on the other hand, implies that near coincidence

$$
\Delta_{\beta^{\prime}}^{\alpha^{\prime}}=\delta_{\beta^{\prime}}^{\alpha^{\prime}}+\frac{1}{6} R_{\gamma^{\prime} \beta^{\prime} \delta^{\prime}}^{\alpha^{\prime}} \sigma^{\gamma^{\prime}} \sigma^{\delta^{\prime}}+\mathcal{O}\left(\epsilon^{3}\right)
$$

so that

$$
\Delta=1+\frac{1}{6} R_{\alpha^{\prime} \beta^{\prime}} \sigma^{\alpha^{\prime}} \sigma^{\beta^{\prime}}+\mathcal{O}\left(\epsilon^{3}\right)
$$


This last result follows from the fact that for a "small" matrix $\boldsymbol{a}, \operatorname{det}(\mathbf{1}+\boldsymbol{a})=1+\operatorname{tr}(\boldsymbol{a})+\mathcal{O}\left(\boldsymbol{a}^{2}\right)$.

We shall prove below that the van Vleck determinant satisfies the differential equation

$$
\frac{1}{\Delta}\left(\Delta \sigma^{\alpha}\right)_{; \alpha}=4
$$

which can also be written as $(\ln \Delta){ }_{,} \sigma^{\alpha}=4-\sigma_{\alpha}^{\alpha}$, or

$$
\frac{d}{d \lambda^{*}}(\ln \Delta)=3-\theta^{*}
$$

in the notation introduced in Section 2.1.4. Equation (99) reveals that the behaviour of the van Vleck determinant is governed by the expansion of the congruence of geodesics that emanate from $x^{\prime}$. If $\theta^{*}<3$, then the congruence expands less rapidly than it would in flat spacetime, and $\Delta$ increases along the geodesics. If, on the other hand, $\theta^{*}>3$, then the congruence expands more rapidly than it would in flat spacetime, and $\Delta$ decreases along the geodesics. Thus, $\Delta>1$ indicates that the geodesics are undergoing focusing, while $\Delta<1$ indicates that the geodesics are undergoing defocusing. The connection between the van Vleck determinant and the strong energy condition is well illustrated by Equation (97): The sign of $\Delta-1$ near $x^{\prime}$ is determined by the sign of $R_{\alpha^{\prime} \beta^{\prime}} \sigma^{\alpha^{\prime}} \sigma^{\beta^{\prime}}$.

\subsubsection{Derivations}

To show that Equation (95) follows from Equation (94) we rewrite the completeness relations at $x$, $g^{\alpha \beta}=\eta^{\mathrm{ab}} e_{\mathrm{a}}^{\alpha} e_{\mathrm{b}}^{\beta}$, in the matrix form $\boldsymbol{g}^{-1}=\boldsymbol{E} \boldsymbol{\eta} \boldsymbol{E}^{T}$, where $\boldsymbol{E}$ denotes the $4 \times 4$ matrix whose entries correspond to $e_{\mathrm{a}}^{\alpha}$. (In this translation we put tensor and frame indices on equal footing.) With $e$ denoting the determinant of this matrix, we have $1 / g=-e^{2}$, or $e=1 / \sqrt{-g}$. Similarly, we rewrite the completeness relations at $x^{\prime}, g^{\alpha^{\prime} \beta^{\prime}}=\eta^{\mathrm{ab}} e_{\mathrm{a}}^{\alpha^{\prime}} e_{\mathrm{b}}^{\beta^{\prime}}$, in the matrix form $\boldsymbol{g}^{\prime-1}=\boldsymbol{E}^{\prime} \boldsymbol{\eta} \boldsymbol{E}^{\boldsymbol{\prime}^{T}}$, where $\boldsymbol{E}^{\prime}$ is the matrix corresponding to $e_{\mathrm{a}}^{\alpha^{\prime}}$. With $e^{\prime}$ denoting its determinant, we have $1 / g^{\prime}=-e^{2}$, or $e^{\prime}=1 / \sqrt{-g^{\prime}}$. Now, the parallel propagator is defined by $g_{\alpha^{\prime}}^{\alpha}=\eta^{\mathrm{ab}} g_{\alpha^{\prime} \beta^{\prime}} e_{\mathrm{a}}^{\alpha} e_{\mathrm{b}}^{\beta^{\prime}}$, and the matrix form of this equation is $\hat{\boldsymbol{g}}=\boldsymbol{E} \boldsymbol{\eta} \boldsymbol{E}^{\prime T} \boldsymbol{g}^{\prime T}$. The determinant of the parallel propagator is therefore $\hat{g}=-e e^{\prime} g^{\prime}=\sqrt{-g^{\prime}} / \sqrt{-g}$. So we have

$$
\operatorname{det}\left[g_{\alpha^{\prime}}^{\alpha}\right]=\frac{\sqrt{-g^{\prime}}}{\sqrt{-g}}, \quad \operatorname{det}\left[g_{\alpha}^{\alpha^{\prime}}\right]=\frac{\sqrt{-g}}{\sqrt{-g^{\prime}}},
$$

and Equation (95) follows from the fact that the matrix form of Equation (94) is $\boldsymbol{\Delta}=-\hat{\boldsymbol{g}}^{-1} \boldsymbol{g}^{-1} \boldsymbol{\sigma}$, where $\boldsymbol{\sigma}$ is the matrix corresponding to $\sigma_{\alpha \beta^{\prime}}$.

To establish Equation (98) we differentiate the relation $\sigma=\frac{1}{2} \sigma^{\gamma} \sigma_{\gamma}$ twice and obtain $\sigma_{\alpha \beta^{\prime}}=$ $\sigma_{\alpha}^{\gamma} \sigma_{\gamma \beta^{\prime}}+\sigma^{\gamma} \sigma_{\gamma \alpha \beta^{\prime}}$. If we replace the last factor by $\sigma_{\alpha \beta^{\prime} \gamma}$ and multiply both sides by $-g^{\alpha^{\prime} \alpha}$ we find

$$
\Delta_{\beta^{\prime}}^{\alpha^{\prime}}=-g^{\alpha^{\prime} \alpha}\left(\sigma_{\alpha}^{\gamma} \sigma_{\gamma \beta^{\prime}}+\sigma^{\gamma} \sigma_{\alpha \beta^{\prime} \gamma}\right)
$$

In this expression we make the substitution $\sigma_{\alpha \beta^{\prime}}=-g_{\alpha \alpha^{\prime}} \Delta_{\beta^{\prime}}^{\alpha^{\prime}}$, which follows directly from Equation (94). This gives us

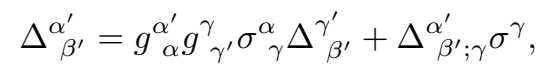

where we have used Equation (78). At this stage we introduce an inverse $\left(\Delta^{-1}\right)^{\alpha^{\prime}}$ to the van Vleck bitensor, defined by $\Delta_{\beta^{\prime}}^{\alpha^{\prime}}\left(\Delta^{-1}\right)_{\gamma^{\prime}}^{\beta^{\prime}}=\delta_{\gamma^{\prime}}^{\alpha^{\prime}}$. After multiplying both sides of Equation (101) by $\left(\Delta^{-1}\right)_{\gamma^{\prime}}^{\beta^{\prime}}$ we find

$$
\delta_{\beta^{\prime}}^{\alpha^{\prime}}=g_{\alpha}^{\alpha^{\prime}} g_{\beta^{\prime}}^{\beta} \sigma_{\beta}^{\alpha}+\left(\Delta^{-1}\right)_{\beta^{\prime}}^{\gamma^{\prime}} \Delta_{\gamma^{\prime} ; \gamma}^{\alpha^{\prime}} \sigma^{\gamma}
$$

Living Reviews in Relativity

http: //www . livingreviews . org//rr-2004-6 
and taking the trace of this equation yields

$$
4=\sigma_{\alpha}^{\alpha}+\left(\Delta^{-1}\right)_{\alpha^{\prime}}^{\beta^{\prime}} \Delta_{\beta^{\prime} ; \gamma}^{\alpha^{\prime}} \sigma^{\gamma} .
$$

We now recall the identity $\delta \ln \operatorname{det} \boldsymbol{M}=\operatorname{tr}\left(\boldsymbol{M}^{-1} \delta \boldsymbol{M}\right)$, which relates the variation of a determinant to the variation of the matrix elements. It implies, in particular, that $\left(\Delta^{-1}\right)_{\alpha^{\prime}}^{\beta^{\prime}} \Delta_{\beta^{\prime} ; \gamma}^{\alpha^{\prime}}=(\ln \Delta)_{, \gamma}$, and we finally obtain

$$
4=\sigma_{\alpha}^{\alpha}+(\ln \Delta)_{, \alpha} \sigma^{\alpha},
$$

which is equivalent to Equation (98) or Equation (99). 


\section{Coordinate Systems}

\subsection{Riemann normal coordinates}

\subsubsection{Definition and coordinate transformation}

Given a fixed base point $x^{\prime}$ and a tetrad $e_{\mathrm{a}}^{\alpha^{\prime}}\left(x^{\prime}\right)$, we assign to a neighbouring point $x$ the four coordinates

$$
\hat{x}^{\mathrm{a}}=-e_{\alpha^{\prime}}^{\mathrm{a}}\left(x^{\prime}\right) \sigma^{\alpha^{\prime}}\left(x, x^{\prime}\right),
$$

where $e_{\alpha^{\prime}}^{\mathrm{a}}=\eta^{\mathrm{ab}} g_{\alpha^{\prime} \beta^{\prime}} e_{\mathrm{b}}^{\beta^{\prime}}$ is the dual tetrad attached to $x^{\prime}$. The new coordinates $\hat{x}^{\mathrm{a}}$ are called Riemann normal coordinates (RNC), and they are such that $\eta_{\mathrm{ab}} \hat{x}^{\mathrm{a}} \hat{x}^{\mathrm{b}}=\eta_{\mathrm{ab}} e_{\alpha^{\prime}}^{\mathrm{a}} e_{\beta^{\prime}}^{\mathrm{b}} \sigma^{\alpha^{\prime}} \sigma^{\beta^{\prime}}=g_{\alpha^{\prime} \beta^{\prime}} \sigma^{\alpha^{\prime}} \sigma^{\beta^{\prime}}$, or

$$
\eta_{\mathrm{ab}} \hat{x}^{\mathrm{a}} \hat{x}^{\mathrm{b}}=2 \sigma\left(x, x^{\prime}\right) .
$$

Thus, $\eta_{\mathrm{ab}} \hat{x}^{\mathrm{a}} \hat{x}^{\mathrm{b}}$ is the squared geodesic distance between $x$ and the base point $x^{\prime}$. It is obvious that $x^{\prime}$ is at the origin of the RNC, where $\hat{x}^{\mathrm{a}}=0$.

If we move the point $x$ to $x+\delta x$, the new coordinates change to $\hat{x}^{\mathrm{a}}+\delta \hat{x}^{\mathrm{a}}=-e_{\alpha^{\prime}}^{\mathrm{a}} \sigma^{\alpha^{\prime}}\left(x+\delta x, x^{\prime}\right)=$ $\hat{x}^{\mathrm{a}}-e_{\alpha^{\prime}}^{\mathrm{a}} \sigma_{\beta}^{\alpha^{\prime}} \delta x^{\beta}$, so that

$$
d \hat{x}^{\mathrm{a}}=-e_{\alpha^{\prime}}^{\mathrm{a}} \sigma_{\beta}^{\alpha^{\prime}} d x^{\beta}
$$

The coordinate transformation is therefore determined by $\partial \hat{x}^{\mathrm{a}} / \partial x^{\beta}=-e_{\alpha^{\prime}}^{\mathrm{a}} \sigma_{\beta}^{\alpha^{\prime}}$, and at coincidence we have

$$
\left[\frac{\partial \hat{x}^{\mathrm{a}}}{\partial x^{\alpha}}\right]=e_{\alpha^{\prime}}^{\mathrm{a}}, \quad\left[\frac{\partial x^{\alpha}}{\partial \hat{x}^{\mathrm{a}}}\right]=e_{\mathrm{a}}^{\alpha^{\prime}}
$$

the second result follows from the identities $e_{\alpha^{\prime}}^{\mathrm{a}} e_{\mathrm{b}}^{\alpha^{\prime}}=\delta_{\mathrm{b}}^{\mathrm{a}}$ and $e_{\mathrm{a}}^{\alpha^{\prime}} e_{\beta^{\prime}}^{\mathrm{a}}=\delta_{\beta^{\prime}}^{\alpha^{\prime}}$.

It is interesting to note that the Jacobian of the transformation of Equation (105), $J \equiv$ $\operatorname{det}\left(\partial \hat{x}^{\mathrm{a}} / \partial x^{\beta}\right)$, is given by $J=\sqrt{-g} \Delta\left(x, x^{\prime}\right)$, where $g$ is the determinant of the metric in the original coordinates, and $\Delta\left(x, x^{\prime}\right)$ is the van Vleck determinant of Equation (95). This result follows simply by writing the coordinate transformation in the form $\partial \hat{x}^{\mathrm{a}} / \partial x^{\beta}=-\eta^{\mathrm{ab}} e_{\mathrm{b}}^{\alpha^{\prime}} \sigma_{\alpha^{\prime} \beta}$ and computing the product of the determinants. It allows us to deduce that in the RNC, the determinant of the metric is given by

$$
\sqrt{-g(\mathrm{RNC})}=\frac{1}{\Delta\left(x, x^{\prime}\right)} .
$$

It is easy to show that the geodesics emanating from $x^{\prime}$ are straight lines in the RNC. The proper volume of a small comoving region is then equal to $d V=\Delta^{-1} d^{4} \hat{x}$, and this is smaller than the flat-spacetime value of $d^{4} \hat{x}$ if $\Delta>1$, that is, if the geodesics are focused by the spacetime curvature.

\subsubsection{Metric near $x^{\prime}$}

We now would like to invert Equation (105) in order to express the line element $d s^{2}=g_{\alpha \beta} d x^{\alpha} d x^{\beta}$ in terms of the displacements $d \hat{x}^{\mathrm{a}}$. We shall do this approximately, by working in a small neighbourhood of $x^{\prime}$. We recall the expansion of Equation (89),

$$
\sigma_{\beta}^{\alpha^{\prime}}=-g_{\beta}^{\beta^{\prime}}\left(\delta_{\beta^{\prime}}^{\alpha^{\prime}}+\frac{1}{6} R_{\gamma^{\prime} \beta^{\prime} \delta^{\prime}}^{\alpha^{\prime}} \sigma^{\gamma^{\prime}} \sigma^{\delta^{\prime}}\right)+\mathcal{O}\left(\epsilon^{3}\right)
$$

and in this we substitute the frame decomposition of the Riemann tensor, $R_{\gamma^{\prime} \beta^{\prime} \delta^{\prime}}^{\alpha^{\prime}}=R_{\text {cbd }}^{\mathrm{a}} e_{\mathrm{a}}^{\alpha^{\prime}} e_{\gamma^{\prime}}^{\mathrm{c}} e_{\beta^{\prime}}^{\mathrm{b}} e_{\delta^{\prime}}^{\mathrm{d}}$, and the tetrad decomposition of the parallel propagator, $g_{\beta}^{\beta^{\prime}}=e_{\mathrm{b}}^{\beta^{\prime}} e_{\beta}^{\mathrm{b}}$, where $e_{\beta}^{\mathrm{b}}(x)$ is the dual tetrad at $x$ obtained by parallel transport of $e_{\beta^{\prime}}^{\mathrm{b}}\left(x^{\prime}\right)$. After some algebra we obtain

$$
\sigma_{\beta}^{\alpha^{\prime}}=-e_{\mathrm{a}}^{\alpha^{\prime}} e_{\beta}^{\mathrm{a}}-\frac{1}{6} R_{\mathrm{cbd}}^{\mathrm{a}} e_{\mathrm{a}}^{\alpha^{\prime}} e_{\beta}^{\mathrm{b}} \hat{x}^{\mathrm{c}} \hat{x}^{\mathrm{d}}+\mathcal{O}\left(\epsilon^{3}\right),
$$

Living Reviews in Relativity

http://www. livingreviews.org/lrr-2004-6 
where we have used Equation (103). Substituting this into Equation (105) yields

$$
d \hat{x}^{\mathrm{a}}=\left[\delta_{\mathrm{b}}^{\mathrm{a}}+\frac{1}{6} R_{\mathrm{cbd}}^{\mathrm{a}} \hat{x}^{\mathrm{c}} \hat{x}^{\mathrm{d}}+\mathcal{O}\left(x^{3}\right)\right] e_{\beta}^{\mathrm{b}} d x^{\beta},
$$

and this is easily inverted to give

$$
e_{\alpha}^{\mathrm{a}} d x^{\alpha}=\left[\delta_{\mathrm{b}}^{\mathrm{a}}-\frac{1}{6} R_{\text {cbd }}^{\mathrm{a}} \hat{x}^{\mathrm{c}} \hat{x}^{\mathrm{d}}+\mathcal{O}\left(x^{3}\right)\right] d \hat{x}^{\mathrm{b}} .
$$

This is the desired approximate inversion of Equation (105). It is useful to note that Equation (109), when specialized from the arbitrary coordinates $x^{\alpha}$ to $\hat{x}^{\mathrm{a}}$, gives us the components of the dual tetrad at $x$ in the RNC.

We are now in a position to calculate the metric in the new coordinates. We have $d s^{2}=$ $g_{\alpha \beta} d x^{\alpha} d x^{\beta}=\left(\eta_{\mathrm{ab}} e_{\alpha}^{\mathrm{a}} e_{\beta}^{\mathrm{b}}\right) d x^{\alpha} d x^{\beta}=\eta_{\mathrm{ab}}\left(e_{\alpha}^{\mathrm{a}} d x^{\alpha}\right)\left(e_{\beta}^{\mathrm{b}} d x^{\beta}\right)$, and in this we substitute Equation (109). The final result is $d s^{2}=g_{\mathrm{ab}} d \hat{x}^{\mathrm{a}} d \hat{x}^{\mathrm{b}}$, with

$$
g_{\mathrm{ab}}=\eta_{\mathrm{ab}}-\frac{1}{3} R_{\mathrm{acbd}} \hat{x}^{\mathrm{c}} \hat{x}^{\mathrm{d}}+\mathcal{O}\left(x^{3}\right) .
$$

The quantities $R_{\text {acbd }}$ appearing in Equation (110) are the frame components of the Riemann tensor evaluated at the base point $x^{\prime}$,

$$
R_{\mathrm{acbd}}=R_{\alpha^{\prime} \gamma^{\prime} \beta^{\prime} \delta^{\prime}} e_{\mathrm{a}}^{\alpha^{\prime}} e_{\mathrm{c}}^{\gamma^{\prime}} e_{\mathrm{b}}^{\beta^{\prime}} e_{\mathrm{d}}^{\delta^{\prime}},
$$

and these are independent of $\hat{x}^{\mathrm{a}}$. They are also, by virtue of Equation (106), the components of the (base-point) Riemann tensor in the RNC, because Equation (111) can also be expressed as

$$
R_{\mathrm{acdb}}=R_{\alpha^{\prime} \gamma^{\prime} \beta^{\prime} \delta^{\prime}}\left[\frac{\partial x^{\alpha}}{\partial \hat{x}^{\mathrm{a}}}\right]\left[\frac{\partial x^{\gamma}}{\partial \hat{x}^{\mathrm{c}}}\right]\left[\frac{\partial x^{\beta}}{\partial \hat{x}^{\mathrm{b}}}\right]\left[\frac{\partial x^{\delta}}{\partial \hat{x}^{\mathrm{d}}}\right],
$$

which is the standard transformation law for tensor components.

It is obvious from Equation (110) that $g_{\mathrm{ab}}\left(x^{\prime}\right)=\eta_{\mathrm{ab}}$ and $\Gamma_{\mathrm{bc}}^{\mathrm{a}}\left(x^{\prime}\right)=0$, where $\Gamma_{\mathrm{bc}}^{\mathrm{a}}=-\frac{1}{3}\left(R_{\mathrm{bcd}}^{\mathrm{a}}+\right.$ $\left.R_{\text {cbd }}^{\mathrm{a}}\right) \hat{x}^{\mathrm{d}}+\mathcal{O}\left(x^{2}\right)$ is the connection compatible with the metric $g_{\mathrm{ab}}$. The Riemann normal coordinates therefore provide a constructive proof of the local flatness theorem.

\subsection{Fermi normal coordinates}

\subsubsection{Fermi-Walker transport}

Let $\gamma$ be a timelike curve described by parametric relations $z^{\mu}(\tau)$ in which $\tau$ is proper time. Let $u^{\mu}=d z^{\mu} / d \tau$ be the curve's normalized tangent vector, and let $a^{\mu}=D u^{\mu} / d \tau$ be its acceleration vector.

A vector field $v^{\mu}$ is said to be Fermi-Walker transported on $\gamma$ if it is a solution to the differential equation

$$
\frac{D v^{\mu}}{d \tau}=\left(v_{\nu} a^{\nu}\right) u^{\mu}-\left(v_{\nu} u^{\nu}\right) a^{\mu} .
$$

Notice that this reduces to parallel transport if $a^{\mu}=0$ and $\gamma$ is a geodesic.

The operation of Fermi-Walker (FW) transport satisfies two important properties. The first is that $u^{\mu}$ is automatically FW transported along $\gamma$; this follows at once from Equation (112) and the fact that $u^{\mu}$ is orthogonal to $a^{\mu}$. The second is that if the vectors $v^{\mu}$ and $w^{\mu}$ are both FW transported along $\gamma$, then their inner product $v_{\mu} w^{\mu}$ is constant on $\gamma: D\left(v_{\mu} w^{\mu}\right) / d \tau=0$; this also follows immediately from Equation (112). 


\subsubsection{Tetrad and dual tetrad on $\gamma$}

Let $\bar{z}$ be an arbitrary reference point on $\gamma$. At this point we erect an orthonormal tetrad $\left(u^{\bar{\mu}}, e_{a}^{\bar{\mu}}\right)$ where, contrary to former usage, the frame index $a$ runs from 1 to 3 . We then propagate each frame vector on $\gamma$ by FW transport; this guarantees that the tetrad remains orthonormal everywhere on $\gamma$. At a generic point $z(\tau)$ we have

$$
\frac{D e_{a}^{\mu}}{d \tau}=\left(a_{\nu} e_{a}^{\nu}\right) u^{\mu}, \quad g_{\mu \nu} u^{\mu} u^{\nu}=-1, \quad g_{\mu \nu} e_{a}^{\mu} u^{\nu}=0, \quad g_{\mu \nu} e_{a}^{\mu} e_{b}^{\nu}=\delta_{a b} .
$$

From the tetrad on $\gamma$ we define a dual tetrad $\left(e_{\mu}^{0}, e_{\mu}^{a}\right)$ by the relations

$$
e_{\mu}^{0}=-u_{\mu}, \quad e_{\mu}^{a}=\delta^{a b} g_{\mu \nu} e_{b}^{\nu} ;
$$

this is also FW transported on $\gamma$. The tetrad and its dual give rise to the completeness relations

$$
g^{\mu \nu}=-u^{\mu} u^{\nu}+\delta^{a b} e_{a}^{\mu} e_{b}^{\nu}, \quad g_{\mu \nu}=-e_{\mu}^{0} e_{\nu}^{0}+\delta_{a b} e_{\mu}^{a} e_{\nu}^{b} .
$$

\subsubsection{Fermi normal coordinates}

To construct the Fermi normal coordinates (FNC) of a point $x$ in the normal convex neighbourhood of $\gamma$, we locate the unique spacelike geodesic $\beta$ that passes through $x$ and intersects $\gamma$ orthogonally. We denote the intersection point by $\bar{x} \equiv z(t)$, with $t$ denoting the value of the proper-time parameter at this point. To tensors at $\bar{x}$ we assign indices $\bar{\alpha}, \bar{\beta}$, and so on. The FNC of $x$ are defined by

$$
\hat{x}^{0}=t, \quad \hat{x}^{a}=-e_{\bar{\alpha}}^{a}(\bar{x}) \sigma^{\bar{\alpha}}(x, \bar{x}), \quad \sigma_{\bar{\alpha}}(x, \bar{x}) u^{\bar{\alpha}}(\bar{x})=0 ;
$$

the last statement determines $\bar{x}$ from the requirement that $-\sigma^{\bar{\alpha}}$, the vector tangent to $\beta$ at $\bar{x}$, be orthogonal to $u^{\bar{\alpha}}$, the vector tangent to $\gamma$. From the definition of the FNC and the completeness relations of Equation (115) it follows that

$$
s^{2} \equiv \delta_{a b} \hat{x}^{a} \hat{x}^{b}=2 \sigma(x, \bar{x}),
$$

so that $s$ is the spatial distance between $\bar{x}$ and $x$ along the geodesic $\beta$. This statement gives an immediate meaning to $\hat{x}^{a}$, the spatial Fermi normal coordinates; and the time coordinate $\hat{x}^{0}$ is simply proper time at the intersection point $\bar{x}$. The situation is illustrated in Figure 6.

Suppose that $x$ is moved to $x+\delta x$. This typically induces a change in the spacelike geodesic $\beta$, which moves to $\beta+\delta \beta$, and a corresponding change in the intersection point $\bar{x}$, which moves to $x^{\prime \prime} \equiv \bar{x}+\delta \bar{x}$, with $\delta x^{\bar{\alpha}}=u^{\bar{\alpha}} \delta t$. The FNC of the new point are then $\hat{x}^{0}(x+\delta x)=t+\delta t$ and $\hat{x}^{a}(x+\delta x)=-e_{\alpha^{\prime \prime}}^{a}\left(x^{\prime \prime}\right) \sigma^{\alpha^{\prime \prime}}\left(x+\delta x, x^{\prime \prime}\right)$, with $x^{\prime \prime}$ determined by $\sigma_{\alpha^{\prime \prime}}\left(x+\delta x, x^{\prime \prime}\right) u^{\alpha^{\prime \prime}}\left(x^{\prime \prime}\right)=0$. Expanding these relations to first order in the displacements, and simplifying using Equations (113), yields

$$
d t=\mu \sigma_{\bar{\alpha} \beta} u^{\bar{\alpha}} d x^{\beta}, \quad d \hat{x}^{a}=-e_{\bar{\alpha}}^{a}\left(\sigma_{\beta}^{\bar{\alpha}}+\mu \sigma_{\bar{\beta}}^{\bar{\alpha}} u^{\bar{\beta}} \sigma_{\beta \bar{\gamma}} u^{\bar{\gamma}}\right) d x^{\beta},
$$

where $\mu$ is determined by $\mu^{-1}=-\left(\sigma_{\bar{\alpha} \bar{\beta}} u^{\bar{\alpha}} u^{\bar{\beta}}+\sigma_{\bar{\alpha}} a^{\bar{\alpha}}\right)$.

\subsubsection{Coordinate displacements near $\gamma$}

The relations of Equation (118) can be expressed as expansions in powers of $s$, the spatial distance from $\bar{x}$ to $x$. For this we use the expansions of Equations (88) and (89), in which we substitute $\sigma^{\bar{\alpha}}=-e_{a}^{\bar{\alpha}} \hat{x}^{a}$ and $g_{\alpha}^{\bar{\alpha}}=u^{\bar{\alpha}} \bar{e}_{\alpha}^{0}+e_{a}^{\bar{\alpha}} \bar{e}_{\alpha}^{a}$, where $\left(\bar{e}_{\alpha}^{0}, \bar{e}_{\alpha}^{a}\right)$ is a dual tetrad at $x$ obtained by parallel transport of $\left(-u_{\bar{\alpha}}, e_{\bar{\alpha}}^{a}\right)$ on the spacelike geodesic $\beta$. After some algebra we obtain

$$
\mu^{-1}=1+a_{a} \hat{x}^{a}+\frac{1}{3} R_{0 c 0 d} \hat{x}^{c} \hat{x}^{d}+\mathcal{O}\left(s^{3}\right),
$$

Living Reviews in Relativity

http://www. livingreviews . org/lrr-2004-6 


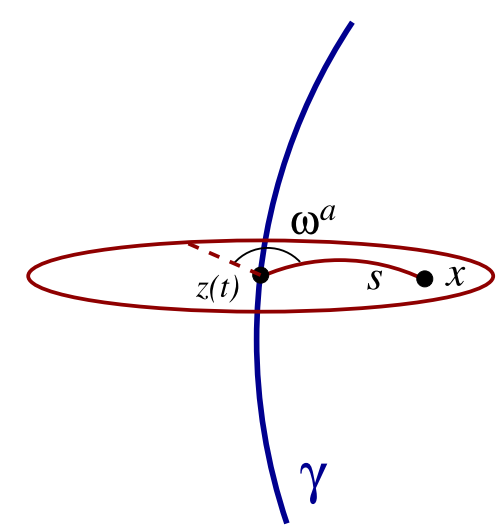

Figure 6: Fermi normal coordinates of a point $x$ relative to a world line $\gamma$. The time coordinate $t$ selects a particular point on the word line, and the disk represents the set of spacelike geodesics that intersect $\gamma$ orthogonally at $z(t)$. The unit vector $\omega^{a} \equiv \hat{x}^{a} / s$ selects a particular geodesic among this set, and the spatial distance $s$ selects a particular point on this geodesic.

where $a_{a}(t) \equiv a_{\bar{\alpha}} e_{a}^{\bar{\alpha}}$ are frame components of the acceleration vector, and $R_{0 c 0 d}(t) \equiv R_{\bar{\alpha} \bar{\gamma} \bar{\beta} \bar{\delta}} u^{\bar{\alpha}} e_{c}^{\bar{\gamma}} u^{\bar{\beta}} e_{d}^{\bar{\delta}}$ are frame components of the Riemann tensor evaluated on $\gamma$. This last result is easily inverted to give

$$
\mu=1-a_{a} \hat{x}^{a}+\left(a_{a} \hat{x}^{a}\right)^{2}-\frac{1}{3} R_{0 c 0 d} \hat{x}^{c} \hat{x}^{d}+\mathcal{O}\left(s^{3}\right) .
$$

Proceeding similarly for the other relations of Equation (118), we obtain

$$
d t=\left[1-a_{a} \hat{x}^{a}+\left(a_{a} \hat{x}^{a}\right)^{2}-\frac{1}{2} R_{0 c 0 d} \hat{x}^{c} \hat{x}^{d}+\mathcal{O}\left(s^{3}\right)\right]\left(\bar{e}_{\beta}^{0} d x^{\beta}\right)+\left[-\frac{1}{6} R_{0 c b d} \hat{x}^{c} \hat{x}^{d}+\mathcal{O}\left(s^{3}\right)\right]\left(\bar{e}_{\beta}^{b} d x^{\beta}\right)
$$

and

$$
d \hat{x}^{a}=\left[\frac{1}{2} R_{c 0 d}^{a} \hat{x}^{c} \hat{x}^{d}+\mathcal{O}\left(s^{3}\right)\right]\left(\bar{e}_{\beta}^{0} d x^{\beta}\right)+\left[\delta_{b}^{a}+\frac{1}{6} R_{c b d}^{a} \hat{x}^{c} \hat{x}^{d}+\mathcal{O}\left(s^{3}\right)\right]\left(\bar{e}_{\beta}^{b} d x^{\beta}\right),
$$

where $R_{a c 0 d}(t) \equiv R_{\bar{\alpha} \bar{\gamma} \bar{\beta} \bar{\delta}} e_{a}^{\bar{\alpha}} e_{c}^{\bar{\gamma}} u^{\bar{\beta}} e_{d}^{\bar{\delta}}$ and $R_{a c b d}(t) \equiv R_{\bar{\alpha} \bar{\gamma} \bar{\beta} \bar{\delta}} e_{a}^{\bar{\alpha}} e_{c}^{\bar{\gamma}} e_{b}^{\bar{\beta}} e_{d}^{\bar{\delta}}$ are additional frame components of the Riemann tensor evaluated on $\gamma$. (Note that frame indices are raised with $\delta^{a b}$.)

As a special case of Equations (119) and (120) we find that

$$
\left.\frac{\partial t}{\partial x^{\alpha}}\right|_{\gamma}=-u_{\bar{\alpha}},\left.\quad \frac{\partial \hat{x}^{a}}{\partial x^{\alpha}}\right|_{\gamma}=e_{\bar{\alpha}}^{a}
$$

because in the limit $x \rightarrow \bar{x}$ the dual tetrad $\left(\bar{e}_{\alpha}^{0}, \bar{e}_{\alpha}^{a}\right)$ at $x$ coincides with the dual tetrad $\left(-u_{\bar{\alpha}}, e_{\bar{\alpha}}^{a}\right)$ at $\bar{x}$. It follows that on $\gamma$, the transformation matrix between the original coordinates $x^{\alpha}$ and the FNC $\left(t, \hat{x}^{a}\right)$ is formed by the Fermi-Walker transported tetrad:

$$
\left.\frac{\partial x^{\alpha}}{\partial t}\right|_{\gamma}=u^{\bar{\alpha}},\left.\quad \frac{\partial x^{\alpha}}{\partial \hat{x}^{a}}\right|_{\gamma}=e_{a}^{\bar{\alpha}}
$$

This implies that the frame components of the acceleration vector $a_{a}(t)$ are also the components of the acceleration vector in the FNC; orthogonality between $u^{\bar{\alpha}}$ and $a^{\bar{\alpha}}$ means that $a_{0}=0$. Similarly, $R_{0 c 0 d}(t), R_{0 c b d}(t)$, and $R_{a c b d}(t)$ are the components of the Riemann tensor (evaluated on $\gamma$ ) in the Fermi normal coordinates. 


\subsubsection{Metric near $\gamma$}

Inversion of Equations (119) and (120) gives

$$
\bar{e}_{\alpha}^{0} d x^{\alpha}=\left[1+a_{a} \hat{x}^{a}+\frac{1}{2} R_{0 c 0 d} \hat{x}^{c} \hat{x}^{d}+\mathcal{O}\left(s^{3}\right)\right] d t+\left[\frac{1}{6} R_{0 c b d} \hat{x}^{c} \hat{x}^{d}+\mathcal{O}\left(s^{3}\right)\right] d \hat{x}^{b}
$$

and

$$
\bar{e}_{\alpha}^{a} d x^{\alpha}=\left[\delta_{b}^{a}-\frac{1}{6} R_{c b d}^{a} \hat{x}^{c} \hat{x}^{d}+\mathcal{O}\left(s^{3}\right)\right] d \hat{x}^{b}+\left[-\frac{1}{2} R_{c 0 d}^{a} \hat{x}^{c} \hat{x}^{d}+\mathcal{O}\left(s^{3}\right)\right] d t .
$$

These relations, when specialized to the FNC, give the components of the dual tetrad at $x$. They can also be used to compute the metric at $x$, after invoking the completeness relations $g_{\alpha \beta}=$ $-\bar{e}_{\alpha}^{0} \bar{e}_{\beta}^{0}+\delta_{a b} \bar{e}_{\alpha}^{a} \bar{e}_{\beta}^{b}$. This gives

$$
d s^{2}=g_{t t} d t^{2}+2 g_{t a} d t d \hat{x}^{a}+g_{a b} d \hat{x}^{a} d \hat{x}^{b}
$$

with

$$
\begin{aligned}
g_{t t} & =-\left[1+2 a_{a} \hat{x}^{a}+\left(a_{a} \hat{x}^{a}\right)^{2}+R_{0 c 0 d} \hat{x}^{c} \hat{x}^{d}+\mathcal{O}\left(s^{3}\right)\right] \\
g_{t a} & =-\frac{2}{3} R_{0 c a d} \hat{x}^{c} \hat{x}^{d}+\mathcal{O}\left(s^{3}\right) \\
g_{a b} & =\delta_{a b}-\frac{1}{3} R_{a c b d} \hat{x}^{c} \hat{x}^{d}+\mathcal{O}\left(s^{3}\right) .
\end{aligned}
$$

This is the metric near $\gamma$ in the Fermi normal coordinates. Recall that $a_{a}(t)$ are the components of the acceleration vector of $\gamma$ - the timelike curve described by $\hat{x}^{a}=0$ - while $R_{0 c 0 d}(t), R_{0 c b d}(t)$, and $R_{a c b d}(t)$ are the components of the Riemann tensor evaluated on $\gamma$.

Notice that on $\gamma$, the metric of Equations $(125,126,127)$ reduces to $g_{t t}=-1$ and $g_{a b}=\delta_{a b}$. On the other hand, the nonvanishing Christoffel symbols (on $\gamma)$ are $\Gamma_{t a}^{t}=\Gamma_{t t}^{a}=a_{a}$; these are zero (and the FNC enforce local flatness on the entire curve) when $\gamma$ is a geodesic.

\subsubsection{Thorne-Hartle coordinates}

The form of the metric can be simplified if the Ricci tensor vanishes on the world line:

$$
R_{\mu \nu}(z)=0
$$

In such circumstances, a transformation from the Fermi normal coordinates $\left(t, \hat{x}^{a}\right)$ to the ThorneHartle coordinates $\left(t, \hat{y}^{a}\right)$ brings the metric to the form

$$
\begin{aligned}
g_{t t} & =-\left[1+2 a_{a} \hat{y}^{a}+\left(a_{a} \hat{y}^{a}\right)^{2}+R_{0 c 0 d} \hat{y}^{c} \hat{y}^{d}+\mathcal{O}\left(s^{3}\right)\right], \\
g_{t a} & =-\frac{2}{3} R_{0 c a d} \hat{y}^{c} \hat{y}^{d}+\mathcal{O}\left(s^{3}\right), \\
g_{a b} & =\delta_{a b}\left(1-R_{0 c 0 d} \hat{y}^{c} \hat{y}^{d}\right)+\mathcal{O}\left(s^{3}\right) .
\end{aligned}
$$

We see that the transformation leaves $g_{t t}$ and $g_{t a}$ unchanged, but that it diagonalizes $g_{a b}$. This metric was first displayed in [58] and the coordinate transformation was later produced by Zhang [64].

The key to the simplification comes from Equation (128), which dramatically reduces the number of independent components of the Riemann tensor. In particular, Equation (128) implies that the frame components $R_{a c b d}$ of the Riemann tensor are completely determined by $\mathcal{E}_{a b} \equiv R_{0 a 0 b}$, which in this special case is a symmetric-tracefree tensor. To prove this we invoke the completeness

Living Reviews in Relativity

http: //www . livingreviews . org/lrr-2004-6 
relations of Equation (115) and take frame components of Equation (128). This produces the three independent equations

$$
\delta^{c d} R_{a c b d}=\mathcal{E}_{a b}, \quad \delta^{c d} R_{0 c a d}=0, \quad \delta^{c d} \mathcal{E}_{c d}=0,
$$

the last of which states that $\mathcal{E}_{a b}$ has a vanishing trace. Taking the trace of the first equation gives $\delta^{a b} \delta^{c d} R_{a c b d}=0$, and this implies that $R_{a c b d}$ has five independent components. Since this is also the number of independent components of $\mathcal{E}_{a b}$, we see that the first equation can be inverted $-R_{a c b d}$ can be expressed in terms of $\mathcal{E}_{a b}$. A complete listing of the relevant relations is $R_{1212}=\mathcal{E}_{11}+\mathcal{E}_{22}=-\mathcal{E}_{33}$, $R_{1213}=\mathcal{E}_{23}, R_{1223}=-\mathcal{E}_{13}, R_{1313}=\mathcal{E}_{11}+\mathcal{E}_{33}=-\mathcal{E}_{22}, R_{1323}=\mathcal{E}_{12}$, and $R_{2323}=\mathcal{E}_{22}+\mathcal{E}_{33}=-\mathcal{E}_{11}$. These are summarized by

$$
R_{a c b d}=\delta_{a b} \mathcal{E}_{c d}+\delta_{c d} \mathcal{E}_{a b}-\delta_{a d} \mathcal{E}_{b c}-\delta_{b c} \mathcal{E}_{a d},
$$

and $\mathcal{E}_{a b} \equiv R_{0 a 0 b}$ satisfies $\delta^{a b} \mathcal{E}_{a b}=0$.

We may also note that the relation $\delta^{c d} R_{0 c a d}=0$, together with the usual symmetries of the Riemann tensor, imply that $R_{0 \text { cad }}$ too possesses five independent components. These may thus be related to another symmetric-tracefree tensor $\mathcal{B}_{a b}$. We take the independent components to be $R_{0112} \equiv-\mathcal{B}_{13}, R_{0113} \equiv \mathcal{B}_{12}, R_{0123} \equiv-\mathcal{B}_{11}, R_{0212} \equiv-\mathcal{B}_{23}$, and $R_{0213} \equiv \mathcal{B}_{22}$, and it is easy to see that all other components can be expressed in terms of these. For example, $R_{0223}=$ $-R_{0113}=-\mathcal{B}_{12}, R_{0312}=-R_{0123}+R_{0213}=\mathcal{B}_{11}+\mathcal{B}_{22}=-\mathcal{B}_{33}, R_{0313}=-R_{0212}=\mathcal{B}_{23}$, and $R_{0323}=R_{0112}=-\mathcal{B}_{13}$. These relations are summarized by

$$
R_{0 a b c}=-\varepsilon_{b c d} \mathcal{B}_{a}^{d},
$$

where $\varepsilon_{a b c}$ is the three-dimensional permutation symbol. The inverse relation is $\mathcal{B}_{b}^{a}=\frac{1}{2} \varepsilon^{a c d} R_{0 b c d}$.

Substitution of Equation (132) into Equation (127) gives

$$
g_{a b}=\delta_{a b}\left(1-\frac{1}{3} \mathcal{E}_{c d} \hat{x}^{c} \hat{x}^{d}\right)-\frac{1}{3}\left(\hat{x}_{c} \hat{x}^{c}\right) \mathcal{E}_{a b}+\frac{1}{3} \hat{x}_{a} \mathcal{E}_{b c} \hat{x}^{c}+\frac{1}{3} \hat{x}_{b} \mathcal{E}_{a c} \hat{x}^{c}+\mathcal{O}\left(s^{3}\right),
$$

and we have not yet achieved the simple form of Equation (131). The missing step is the transformation from the FNC $\hat{x}^{a}$ to the Thorne-Hartle coordinates $\hat{y}^{a}$. This is given by

$$
\hat{y}^{a}=\hat{x}^{a}+\xi^{a}, \quad \xi^{a}=-\frac{1}{6}\left(\hat{x}_{c} \hat{x}^{c}\right) \mathcal{E}_{a b} \hat{x}^{b}+\frac{1}{3} \hat{x}_{a} \mathcal{E}_{b c} \hat{x}^{b} \hat{x}^{c}+\mathcal{O}\left(s^{4}\right) .
$$

It is easy to see that this transformation affects neither $g_{t t}$ nor $g_{t a}$ at orders $s$ and $s^{2}$. The remaining components of the metric, however, transform according to $g_{a b}(\mathrm{THC})=g_{a b}(\mathrm{FNC})-\xi_{a ; b}-\xi_{b ; a}$, where

$$
\xi_{a ; b}=\frac{1}{3} \delta_{a b} \mathcal{E}_{c d} \hat{x}^{c} \hat{x}^{d}-\frac{1}{6}\left(\hat{x}_{c} \hat{x}^{c}\right) \mathcal{E}_{a b}-\frac{1}{3} \mathcal{E}_{a c} \hat{x}^{c} \hat{x}_{b}+\frac{2}{3} \hat{x}_{a} \mathcal{E}_{b c} \hat{x}^{c}+\mathcal{O}\left(s^{3}\right) .
$$

It follows that $g_{a b}^{\mathrm{THC}}=\delta_{a b}\left(1-\mathcal{E}_{c d} \hat{y}^{c} \hat{y}^{d}\right)+\mathcal{O}\left(\hat{y}^{3}\right)$, which is just the same statement as in Equation (131).

Alternative expressions for the components of the Thorne-Hartle metric are

$$
\begin{aligned}
g_{t t} & =-\left[1+2 a_{a} \hat{y}^{a}+\left(a_{a} \hat{y}^{a}\right)^{2}+\mathcal{E}_{a b} \hat{y}^{a} \hat{y}^{b}+\mathcal{O}\left(s^{3}\right)\right], \\
g_{t a} & =-\frac{2}{3} \varepsilon_{a b c} \mathcal{B}^{b} \hat{y}^{c} \hat{y}^{d}+\mathcal{O}\left(s^{3}\right), \\
g_{a b} & =\delta_{a b}\left(1-\mathcal{E}_{c d} \hat{y}^{c} \hat{y}^{d}\right)+\mathcal{O}\left(s^{3}\right) .
\end{aligned}
$$




\subsection{Retarded coordinates}

\subsubsection{Geometrical elements}

We introduce the same geometrical elements as in Section 3.2: We have a timelike curve $\gamma$ described by relations $z^{\mu}(\tau)$, its normalized tangent vector $u^{\mu}=d z^{\mu} / d \tau$, and its acceleration vector $a^{\mu}=$ $D u^{\mu} / d \tau$. We also have an orthonormal triad $e_{a}^{\mu}$ that is transported on the world line according to

$$
\frac{D e_{a}^{\mu}}{d \tau}=a_{a} u^{\mu}+\omega_{a}^{b} e_{b}^{\mu},
$$

where $a_{a}(\tau)=a_{\mu} e_{a}^{\mu}$ are the frame components of the acceleration vector and $\omega_{a b}(\tau)=-\omega_{b a}(\tau)$ is a prescribed rotation tensor. Here the triad is not Fermi-Walker transported: For added generality we allow the spatial vectors to rotate as they are transported on the world line. While $\omega_{a b}$ will be set to zero in most sections of this paper, the freedom to perform such a rotation can be useful and will be exploited in Section 5.4. It is easy to check that Equation (138) is compatible with the requirement that the tetrad $\left(u^{\mu}, e_{a}^{\mu}\right)$ be orthonormal everywhere on $\gamma$. Finally, we have a dual tetrad $\left(e_{\mu}^{0}, e_{\mu}^{a}\right)$, with $e_{\mu}^{0}=-u_{\mu}$ and $e_{\mu}^{a}=\delta^{a b} g_{\mu \nu} e_{b}^{\nu}$. The tetrad and its dual give rise to the completeness relations

$$
g^{\mu \nu}=-u^{\mu} u^{\nu}+\delta^{a b} e_{a}^{\mu} e_{b}^{\nu}, \quad g_{\mu \nu}=-e_{\mu}^{0} e_{\nu}^{0}+\delta_{a b} e_{\mu}^{a} e_{\nu}^{b}
$$

which are the same as in Equation (115).

The Fermi normal coordinates of Section 3.2 were constructed on the basis of a spacelike geodesic connecting a field point $x$ to the world line. The retarded coordinates are based instead on a null geodesic going from the world line to the field point. We thus let $x$ be within the normal convex neighbourhood of $\gamma, \beta$ be the unique future-directed null geodesic that goes from the world line to $x$, and $x^{\prime} \equiv z(u)$ be the point at which $\beta$ intersects the world line, with $u$ denoting the value of the proper-time parameter at this point.

From the tetrad at $x^{\prime}$ we obtain another tetrad $\left(e_{0}^{\alpha}, e_{a}^{\alpha}\right)$ at $x$ by parallel transport on $\beta$. By raising the frame index and lowering the vectorial index we also obtain a dual tetrad at $x: e_{\alpha}^{0}=$ $-g_{\alpha \beta} e_{0}^{\beta}$ and $e_{\alpha}^{a}=\delta^{a b} g_{\alpha \beta} e_{b}^{\beta}$. The metric at $x$ can be then be expressed as

$$
g_{\alpha \beta}=-e_{\alpha}^{0} e_{\beta}^{0}+\delta_{a b} e_{\alpha}^{a} e_{\beta}^{b},
$$

and the parallel propagator from $x^{\prime}$ to $x$ is given by

$$
g_{\alpha^{\prime}}^{\alpha}\left(x, x^{\prime}\right)=-e_{0}^{\alpha} u_{\alpha^{\prime}}+e_{a}^{\alpha} e_{\alpha^{\prime}}^{a}, \quad g_{\alpha}^{\alpha^{\prime}}\left(x^{\prime}, x\right)=u^{\alpha^{\prime}} e_{\alpha}^{0}+e_{a}^{\alpha^{\prime}} e_{\alpha}^{a} .
$$

\subsubsection{Definition of the retarded coordinates}

The quasi-Cartesian version of the retarded coordinates are defined by

$$
\hat{x}^{0}=u, \quad \hat{x}^{a}=-e_{\alpha^{\prime}}^{a}\left(x^{\prime}\right) \sigma^{\alpha^{\prime}}\left(x, x^{\prime}\right), \quad \sigma\left(x, x^{\prime}\right)=0 ;
$$

the last statement indicates that $x^{\prime}$ and $x$ are linked by a null geodesic. From the fact that $\sigma^{\alpha^{\prime}}$ is a null vector we obtain

$$
r \equiv\left(\delta_{a b} \hat{x}^{a} \hat{x}^{b}\right)^{1 / 2}=u_{\alpha^{\prime}} \sigma^{\alpha^{\prime}},
$$

and $r$ is a positive quantity by virtue of the fact that $\beta$ is a future-directed null geodesic - this makes $\sigma^{\alpha^{\prime}}$ past-directed. In flat spacetime, $\sigma^{\alpha^{\prime}}=-\left(x-x^{\prime}\right)^{\alpha}$, and in a Lorentz frame that is momentarily comoving with the world line, $r=t-t^{\prime}>0$; with the speed of light set equal to unity, $r$ is also the spatial distance between $x^{\prime}$ and $x$ as measured in this frame. In curved

Living Reviews in Relativity

http: //www . livingreviews . org/lrr-2004-6 
spacetime, the quantity $r=u_{\alpha^{\prime}} \sigma^{\alpha^{\prime}}$ can still be called the retarded distance between the point $x$ and the world line. Another consequence of Equation (142) is that

$$
\sigma^{\alpha^{\prime}}=-r\left(u^{\alpha^{\prime}}+\Omega^{a} e_{a}^{\alpha^{\prime}}\right)
$$

where $\Omega^{a} \equiv \hat{x}^{a} / r$ is a spatial vector that satisfies $\delta_{a b} \Omega^{a} \Omega^{b}=1$.

A straightforward calculation reveals that under a displacement of the point $x$, the retarded coordinates change according to

$$
d u=-k_{\alpha} d x^{\alpha}, \quad d \hat{x}^{a}=-\left(r a^{a}-\omega_{b}^{a} \hat{x}^{b}+e_{\alpha^{\prime}}^{a} \sigma_{\beta^{\prime}}^{\alpha^{\prime}} u^{\beta^{\prime}}\right) d u-e_{\alpha^{\prime}}^{a} \sigma_{\beta}^{\alpha^{\prime}} d x^{\beta},
$$

where $k_{\alpha}=\sigma_{\alpha} / r$ is a future-directed null vector at $x$ that is tangent to the geodesic $\beta$. To obtain these results we must keep in mind that a displacement of $x$ typically induces a simultaneous displacement of $x^{\prime}$ because the new points $x+\delta x$ and $x^{\prime}+\delta x^{\prime}$ must also be linked by a null geodesic. We therefore have $0=\sigma\left(x+\delta x, x^{\prime}+\delta x^{\prime}\right)=\sigma_{\alpha} \delta x^{\alpha}+\sigma_{\alpha^{\prime}} \delta x^{\alpha^{\prime}}$, and the first relation of Equation (145) follows from the fact that a displacement along the world line is described by $\delta x^{\alpha^{\prime}}=u^{\alpha^{\prime}} \delta u$.

\subsubsection{The scalar field $r(x)$ and the vector field $\boldsymbol{k}^{\alpha}(x)$}

If we keep $x^{\prime}$ linked to $x$ by the relation $\sigma\left(x, x^{\prime}\right)=0$, then the quantity

$$
r(x)=\sigma_{\alpha^{\prime}}\left(x, x^{\prime}\right) u^{\alpha^{\prime}}\left(x^{\prime}\right)
$$

can be viewed as an ordinary scalar field defined in a neighbourhood of $\gamma$. We can compute the gradient of $r$ by finding how $r$ changes under a displacement of $x$ (which again induces a displacement of $x^{\prime}$ ). The result is

$$
\partial_{\beta} r=-\left(\sigma_{\alpha^{\prime}} a^{\alpha^{\prime}}+\sigma_{\alpha^{\prime} \beta^{\prime}} u^{\alpha^{\prime}} u^{\beta^{\prime}}\right) k_{\beta}+\sigma_{\alpha^{\prime} \beta} u^{\alpha^{\prime}} .
$$

Similarly, we can view

$$
k^{\alpha}(x)=\frac{\sigma^{\alpha}\left(x, x^{\prime}\right)}{r(x)}
$$

as an ordinary vector field, which is tangent to the congruence of null geodesics that emanate from $x^{\prime}$. It is easy to check that this vector satisfies the identities

$$
\sigma_{\alpha \beta} k^{\beta}=k_{\alpha}, \quad \sigma_{\alpha^{\prime} \beta} k^{\beta}=\frac{\sigma_{\alpha^{\prime}}}{r},
$$

from which we also obtain $\sigma_{\alpha^{\prime} \beta} u^{\alpha^{\prime}} k^{\beta}=1$. From this last result and Equation (147) we deduce the important relation

$$
k^{\alpha} \partial_{\alpha} r=1 .
$$

In addition, combining the general statement $\sigma^{\alpha}=-g_{\alpha^{\prime}}^{\alpha} \sigma^{\alpha^{\prime}}$ (cf. Equation (79)) with Equation (144) gives

$$
k^{\alpha}=g_{\alpha^{\prime}}^{\alpha}\left(u^{\alpha^{\prime}}+\Omega^{a} e_{a}^{\alpha^{\prime}}\right)
$$

the vector at $x$ is therefore obtained by parallel transport of $u^{\alpha^{\prime}}+\Omega^{a} e_{a}^{\alpha^{\prime}}$ on $\beta$. From this and Equation (141) we get the alternative expression

$$
k^{\alpha}=e_{0}^{\alpha}+\Omega^{a} e_{a}^{\alpha}
$$


which confirms that $k^{\alpha}$ is a future-directed null vector field (recall that $\Omega^{a}=\hat{x}^{a} / r$ is a unit vector).

The covariant derivative of $k_{\alpha}$ can be computed by finding how the vector changes under a displacement of $x$. (It is in fact easier to first calculate how $r k_{\alpha}$ changes, and then substitute our previous expression for $\partial_{\beta} r$.) The result is

$$
r k_{\alpha ; \beta}=\sigma_{\alpha \beta}-k_{\alpha} \sigma_{\beta \gamma^{\prime}} u^{\gamma^{\prime}}-k_{\beta} \sigma_{\alpha \gamma^{\prime}} u^{\gamma^{\prime}}+\left(\sigma_{\alpha^{\prime}} a^{\alpha^{\prime}}+\sigma_{\alpha^{\prime} \beta^{\prime}} u^{\alpha^{\prime}} u^{\beta^{\prime}}\right) k_{\alpha} k_{\beta} .
$$

From this we infer that $k^{\alpha}$ satisfies the geodesic equation in affine-parameter form, $k_{; \beta}^{\alpha} k^{\beta}=0$, and Equation (150) informs us that the affine parameter is in fact $r$. A displacement along a member of the congruence is therefore given by $d x^{\alpha}=k^{\alpha} d r$. Specializing to retarded coordinates, and using Equations (145) and (149), we find that this statement becomes $d u=0$ and $d \hat{x}^{a}=\left(\hat{x}^{a} / r\right) d r$, which integrate to $u=$ const. and $\hat{x}^{a}=r \Omega^{a}$, respectively, with $\Omega^{a}$ still denoting a constant unit vector. We have found that the congruence of null geodesics emanating from $x^{\prime}$ is described by

$$
u=\text { const. }, \quad \hat{x}^{a}=r \Omega^{a}\left(\theta^{A}\right)
$$

in the retarded coordinates. Here, the two angles $\theta^{A}(A=1,2)$ serve to parameterize the unit vector $\Omega^{a}$, which is independent of $r$.

Equation (153) also implies that the expansion of the congruence is given by

$$
\theta=k_{; \alpha}^{\alpha}=\frac{\sigma_{\alpha}^{\alpha}-2}{r}
$$

Using the expansion for $\sigma_{\alpha}^{\alpha}$ given by Equation (91), we find that this becomes $r \theta=2-\frac{1}{3} R_{\alpha^{\prime} \beta^{\prime}} \sigma^{\alpha^{\prime}} \sigma^{\beta^{\prime}}+$ $\mathcal{O}\left(r^{3}\right)$, or

$$
r \theta=2-\frac{1}{3} r^{2}\left(R_{00}+2 R_{0 a} \Omega^{a}+R_{a b} \Omega^{a} \Omega^{b}\right)+\mathcal{O}\left(r^{3}\right)
$$

after using Equation (144). Here, $R_{00}=R_{\alpha^{\prime} \beta^{\prime}} u^{\alpha^{\prime}} u^{\beta^{\prime}}, R_{0 a}=R_{\alpha^{\prime} \beta^{\prime}} u^{\alpha^{\prime}} e_{a}^{\beta^{\prime}}$, and $R_{a b}=R_{\alpha^{\prime} \beta^{\prime}} e_{a}^{\alpha^{\prime}} e_{b}^{\beta^{\prime}}$ are the frame components of the Ricci tensor evaluated at $x^{\prime}$. This result confirms that the congruence is singular at $r=0$, because $\theta$ diverges as $2 / r$ in this limit; the caustic coincides with the point $x^{\prime}$.

Finally, we infer from Equation (153) that $k^{\alpha}$ is hypersurface orthogonal. This, together with the property that $k^{\alpha}$ satisfies the geodesic equation in affine-parameter form, implies that there exists a scalar field $u(x)$ such that

$$
k_{\alpha}=-\partial_{\alpha} u .
$$

This scalar field was already identified in Equation (145): It is numerically equal to the propertime parameter of the world line at $x^{\prime}$. We conclude that the geodesics to which $k^{\alpha}$ is tangent are the generators of the null cone $u=$ const. As Equation (154) indicates, a specific generator is selected by choosing a direction $\Omega^{a}$ (which can be parameterized by two angles $\theta^{A}$ ), and $r$ is an affine parameter on each generator. The geometrical meaning of the retarded coordinates is now completely clear; it is illustrated in Figure 7.

\subsubsection{Frame components of tensor fields on the world line}

The metric at $x$ in the retarded coordinates will be expressed in terms of frame components of vectors and tensors evaluated on the world line $\gamma$. For example, if $a^{\alpha^{\prime}}$ is the acceleration vector at $x^{\prime}$, then as we have seen,

$$
a_{a}(u)=a_{\alpha^{\prime}} e_{a}^{\alpha^{\prime}}
$$

are the frame components of the acceleration at proper time $u$.

Living Reviews in Relativity

http: //www . livingreviews . org/lrr-2004-6 


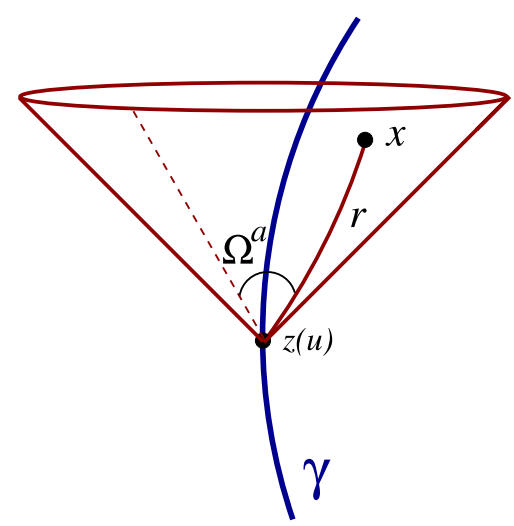

Figure 7: Retarded coordinates of a point $x$ relative to a world line $\gamma$. The retarded time $u$ selects a particular null cone, the unit vector $\Omega^{a} \equiv \hat{x}^{a} / r$ selects a particular generator of this null cone, and the retarded distance $r$ selects a particular point on this generator. This figure is identical to Figure 4.

Similarly,

$$
\begin{aligned}
& R_{a 0 b 0}(u)=R_{\alpha^{\prime} \gamma^{\prime} \beta^{\prime} \delta^{\prime}} e_{a}^{\alpha^{\prime}} u^{\gamma^{\prime}} e_{b}^{\beta^{\prime}} u^{\delta^{\prime}}, \\
& R_{a 0 b d}(u)=R_{\alpha^{\prime} \gamma^{\prime} \beta^{\prime} \delta^{\prime}} e_{a}^{\alpha^{\prime}} u^{\gamma^{\prime}} e_{b}^{\beta^{\prime}} e_{d}^{\delta^{\prime}}, \\
& R_{a c b d}(u)=R_{\alpha^{\prime} \gamma^{\prime} \beta^{\prime} \delta^{\prime}} e_{a}^{\alpha^{\prime}} e_{c}^{\gamma^{\prime}} e_{b}^{\beta^{\prime}} e_{d}^{\delta^{\prime}}
\end{aligned}
$$

are the frame components of the Riemann tensor evaluated on $\gamma$. From these we form the useful combinations

$$
\begin{aligned}
S_{a b}\left(u, \theta^{A}\right) & =R_{a 0 b 0}+R_{a 0 b c} \Omega^{c}+R_{b 0 a c} \Omega^{c}+R_{a c b d} \Omega^{c} \Omega^{d}=S_{b a}, \\
S_{a}\left(u, \theta^{A}\right) & =S_{a b} \Omega^{b}=R_{a 0 b 0} \Omega^{b}-R_{a b 0 c} \Omega^{b} \Omega^{c}, \\
S\left(u, \theta^{A}\right) & =S_{a} \Omega^{a}=R_{a 0 b 0} \Omega^{a} \Omega^{b},
\end{aligned}
$$

in which the quantities $\Omega^{a} \equiv \hat{x}^{a} / r$ depend on the angles $\theta^{A}$ only - they are independent of $u$ and $r$.

We have previously introduced the frame components of the Ricci tensor in Equation (156). The identity

$$
R_{00}+2 R_{0 a} \Omega^{a}+R_{a b} \Omega^{a} \Omega^{b}=\delta^{a b} S_{a b}-S
$$

follows easily from Equations $(160,161,162)$ and the definition of the Ricci tensor.

In Section 3.2 we saw that the frame components of a given tensor were also the components of this tensor (evaluated on the world line) in the Fermi normal coordinates. We should not expect this property to be true also in the case of the retarded coordinates: the frame components of a tensor are not to be identified with the components of this tensor in the retarded coordinates. The reason is that the retarded coordinates are in fact singular on the world line. As we shall see, they give rise to a metric that possesses a directional ambiguity at $r=0$. (This can easily be seen in Minkowski spacetime by performing the coordinate transformation $u=t-\sqrt{x^{2}+y^{2}+z^{2}}$.) Components of tensors are therefore not defined on the world line, although they are perfectly well defined for $r \neq 0$. Frame components, on the other hand, are well defined both off and on the world line, and working with them will eliminate any difficulty associated with the singular nature of the retarded coordinates. 


\subsubsection{Coordinate displacements near $\gamma$}

The changes in the quasi-Cartesian retarded coordinates under a displacement of $x$ are given by Equation (145). In these we substitute the standard expansions for $\sigma_{\alpha^{\prime} \beta^{\prime}}$ and $\sigma_{\alpha^{\prime} \beta}$, as given by Equations (88) and (89), as well as Equations (144) and (151). After a straightforward (but fairly lengthy) calculation, we obtain the following expressions for the coordinate displacements:

$$
\begin{aligned}
d u= & \left(e_{\alpha}^{0} d x^{\alpha}\right)-\Omega_{a}\left(e_{\alpha}^{b} d x^{\alpha}\right) \\
d \hat{x}^{a}= & -\left[r a^{a}-r \omega^{a}{ }_{b} \Omega^{b}+\frac{1}{2} r^{2} S^{a}+\mathcal{O}\left(r^{3}\right)\right]\left(e_{\alpha}^{0} d x^{\alpha}\right) \\
& +\left[\delta^{a}{ }_{b}+\left(r a^{a}-r \omega^{a}{ }_{c} \Omega^{c}+\frac{1}{3} r^{2} S^{a}\right) \Omega_{b}+\frac{1}{6} r^{2} S_{b}^{a}+\mathcal{O}\left(r^{3}\right)\right]\left(e_{\alpha}^{b} d x^{\alpha}\right) .
\end{aligned}
$$

Notice that the result for $d u$ is exact, but that $d \hat{x}^{a}$ is expressed as an expansion in powers of $r$.

These results can also be expressed in the form of gradients of the retarded coordinates:

$$
\begin{aligned}
\partial_{\alpha} u= & e_{\alpha}^{0}-\Omega_{a} e_{\alpha}^{a}, \\
\partial_{\alpha} \hat{x}^{a}= & -\left[r a^{a}-r \omega^{a}{ }_{b} \Omega^{b}+\frac{1}{2} r^{2} S^{a}+\mathcal{O}\left(r^{3}\right)\right] e_{\alpha}^{0} \\
& +\left[\delta_{b}^{a}+\left(r a^{a}-r \omega^{a}{ }_{c} \Omega^{c}+\frac{1}{3} r^{2} S^{a}\right) \Omega_{b}+\frac{1}{6} r^{2} S_{b}^{a}+\mathcal{O}\left(r^{3}\right)\right] e_{\alpha}^{b} .
\end{aligned}
$$

Notice that Equation (166) follows immediately from Equations (152) and (157). From Equation (167) and the identity $\partial_{\alpha} r=\Omega_{a} \partial_{\alpha} \hat{x}^{a}$ we also infer

$$
\partial_{\alpha} r=-\left[r a_{a} \Omega^{a}+\frac{1}{2} r^{2} S+\mathcal{O}\left(r^{3}\right)\right] e_{\alpha}^{0}+\left[\left(1+r a_{b} \Omega^{b}+\frac{1}{3} r^{2} S\right) \Omega_{a}+\frac{1}{6} r^{2} S_{a}+\mathcal{O}\left(r^{3}\right)\right] e_{\alpha}^{a},
$$

where we have used the facts that $S_{a}=S_{a b} \Omega^{b}$ and $S=S_{a} \Omega^{a}$; these last results were derived in Equations (161) and (162). It may be checked that Equation (168) agrees with Equation (147).

\subsubsection{Metric near $\gamma$}

It is straightforward (but fairly tedious) to invert the relations of Equations (164) and (165) and solve for $e_{\alpha}^{0} d x^{\alpha}$ and $e_{\alpha}^{a} d x^{\alpha}$. The results are

$$
\begin{aligned}
& e_{\alpha}^{0} d x^{\alpha}=\left[1+r a_{a} \Omega^{a}+\frac{1}{2} r^{2} S+\mathcal{O}\left(r^{3}\right)\right] d u+\left[\left(1+\frac{1}{6} r^{2} S\right) \Omega_{a}-\frac{1}{6} r^{2} S_{a}+\mathcal{O}\left(r^{3}\right)\right] d \hat{x}^{a}, \\
& e_{\alpha}^{a} d x^{\alpha}=\left[r\left(a^{a}-\omega^{a}{ }_{b} \Omega^{b}\right)+\frac{1}{2} r^{2} S^{a}+\mathcal{O}\left(r^{3}\right)\right] d u+\left[\delta^{a}{ }_{b}-\frac{1}{6} r^{2} S_{b}^{a}+\frac{1}{6} r^{2} S^{a} \Omega_{b}+\mathcal{O}\left(r^{3}\right)\right] d \hat{x}^{b} .
\end{aligned}
$$

These relations, when specialized to the retarded coordinates, give us the components of the dual tetrad $\left(e_{\alpha}^{0}, e_{\alpha}^{a}\right)$ at $x$. The metric is then computed by using the completeness relations of Equation (140). We find

$$
d s^{2}=g_{u u} d u^{2}+2 g_{u a} d u d \hat{x}^{a}+g_{a b} d \hat{x}^{a} d \hat{x}^{b},
$$

with

$$
\begin{aligned}
g_{u u} & =-\left(1+r a_{a} \Omega^{a}\right)^{2}+r^{2}\left(a_{a}-\omega_{a b} \Omega^{b}\right)\left(a^{a}-\omega_{c}^{a} \Omega^{c}\right)-r^{2} S+\mathcal{O}\left(r^{3}\right), \\
g_{u a} & =-\left(1+r a_{b} \Omega^{b}+\frac{2}{3} r^{2} S\right) \Omega_{a}+r\left(a_{a}-\omega_{a b} \Omega^{b}\right)+\frac{2}{3} r^{2} S_{a}+\mathcal{O}\left(r^{3}\right), \\
g_{a b} & =\delta_{a b}-\left(1+\frac{1}{3} r^{2} S\right) \Omega_{a} \Omega_{b}-\frac{1}{3} r^{2} S_{a b}+\frac{1}{3} r^{2}\left(S_{a} \Omega_{b}+\Omega_{a} S_{b}\right)+\mathcal{O}\left(r^{3}\right) .
\end{aligned}
$$

Living Reviews in Relativity

http: //www . livingreviews . org/lrr-2004-6 
We see (as was pointed out in Section 3.3.4) that the metric possesses a directional ambiguity on the world line: The metric at $r=0$ still depends on the vector $\Omega^{a}=\hat{x}^{a} / r$ that specifies the direction to the point $x$. The retarded coordinates are therefore singular on the world line, and tensor components cannot be defined on $\gamma$.

By setting $S_{a b}=S_{a}=S=0$ in Equations $(171,172,173)$ we obtain the metric of flat spacetime in the retarded coordinates. This we express as

$$
\begin{aligned}
\eta_{u u} & =-\left(1+r a_{a} \Omega^{a}\right)^{2}+r^{2}\left(a_{a}-\omega_{a b} \Omega^{b}\right)\left(a^{a}-\omega^{a}{ }_{c} \Omega^{c}\right), \\
\eta_{u a} & =-\left(1+r a_{b} \Omega^{b}\right) \Omega_{a}+r\left(a_{a}-\omega_{a b} \Omega^{b}\right), \\
\eta_{a b} & =\delta_{a b}-\Omega_{a} \Omega_{b} .
\end{aligned}
$$

In spite of the directional ambiguity, the metric of flat spacetime has a unit determinant everywhere, and it is easily inverted:

$$
\eta^{u u}=0, \quad \eta^{u a}=-\Omega^{a}, \quad \eta^{a b}=\delta^{a b}+r\left(a^{a}-\omega_{c}^{a} \Omega^{c}\right) \Omega^{b}+r \Omega^{a}\left(a^{b}-\omega^{b}{ }_{c} \Omega^{c}\right) .
$$

The inverse metric also is ambiguous on the world line.

To invert the curved-spacetime metric of Equations $(171,172,173)$ we express it as $g_{\alpha \beta}=$ $\eta_{\alpha \beta}+h_{\alpha \beta}+\mathcal{O}\left(r^{3}\right)$ and treat $h_{\alpha \beta}=\mathcal{O}\left(r^{2}\right)$ as a perturbation. The inverse metric is then $g^{\alpha \beta}=$ $\eta^{\alpha \beta}-\eta^{\alpha \gamma} \eta^{\beta \delta} h_{\gamma \delta}+\mathcal{O}\left(r^{3}\right)$, or

$$
\begin{aligned}
g^{u u} & =0, \\
g^{u a} & =-\Omega^{a}, \\
g^{a b} & =\delta^{a b}+r\left(a^{a}-\omega^{a}{ }_{c} \Omega^{c}\right) \Omega^{b}+r \Omega^{a}\left(a^{b}-\omega^{b}{ }_{c} \Omega^{c}\right)+\frac{1}{3} r^{2} S^{a b}+\frac{1}{3} r^{2}\left(S^{a} \Omega^{b}+\Omega^{a} S^{b}\right)+\mathcal{O}\left(r^{3}\right) .
\end{aligned}
$$

The results for $g^{u u}$ and $g^{u a}$ are exact, and they follow from the general relations $g^{\alpha \beta}\left(\partial_{\alpha} u\right)\left(\partial_{\beta} u\right)=0$ and $g^{\alpha \beta}\left(\partial_{\alpha} u\right)\left(\partial_{\beta} r\right)=-1$ that are derived from Equations (150) and (157).

The metric determinant is computed from $\sqrt{-g}=1+\frac{1}{2} \eta^{\alpha \beta} h_{\alpha \beta}+\mathcal{O}\left(r^{3}\right)$, which gives

$$
\sqrt{-g}=1-\frac{1}{6} r^{2}\left(\delta^{a b} S_{a b}-S\right)+\mathcal{O}\left(r^{3}\right)=1-\frac{1}{6} r^{2}\left(R_{00}+2 R_{0 a} \Omega^{a}+R_{a b} \Omega^{a} \Omega^{b}\right)+\mathcal{O}\left(r^{3}\right),
$$

where we have substituted the identity of Equation (163). Comparison with Equation (156) then gives us the interesting relation $\sqrt{-g}=\frac{1}{2} r \theta+\mathcal{O}\left(r^{3}\right)$, where $\theta$ is the expansion of the generators of the null cones $u=$ const.

\subsubsection{Transformation to angular coordinates}

Because the vector $\Omega^{a}=\hat{x}^{a} / r$ satisfies $\delta_{a b} \Omega^{a} \Omega^{b}=1$, it can be parameterized by two angles $\theta^{A}$. A canonical choice for the parameterization is $\Omega^{a}=(\sin \theta \cos \phi, \sin \theta \sin \phi, \cos \theta)$. It is then convenient to perform a coordinate transformation from $\hat{x}^{a}$ to $\left(r, \theta^{A}\right)$, using the relations $\hat{x}^{a}=r \Omega^{a}\left(\theta^{A}\right)$. (Recall from Section 3.3.3 that the angles $\theta^{A}$ are constant on the generators of the null cones $u=$ const., and that $r$ is an affine parameter on these generators. The relations $\hat{x}^{a}=r \Omega^{a}$ therefore describe the behaviour of the generators.) The differential form of the coordinate transformation is

$$
d \hat{x}^{a}=\Omega^{a} d r+r \Omega_{A}^{a} d \theta^{A}
$$

where the transformation matrix

$$
\Omega_{A}^{a} \equiv \frac{\partial \Omega^{a}}{\partial \theta^{A}}
$$

satisfies the identity $\Omega_{a} \Omega_{A}^{a}=0$. 
We introduce the quantities

$$
\Omega_{A B}=\delta_{a b} \Omega_{A}^{a} \Omega_{B}^{b},
$$

which act as a (nonphysical) metric in the subspace spanned by the angular coordinates. In the canonical parameterization, $\Omega_{A B}=\operatorname{diag}\left(1, \sin ^{2} \theta\right)$. We use the inverse of $\Omega_{A B}$, denoted $\Omega^{A B}$, to raise upper-case latin indices. We then define the new object

$$
\Omega_{a}^{A}=\delta_{a b} \Omega^{A B} \Omega_{B}^{b}
$$

which satisfies the identities

$$
\Omega_{a}^{A} \Omega_{B}^{a}=\delta_{B}^{A}, \quad \Omega_{A}^{a} \Omega_{b}^{A}=\delta_{b}^{a}-\Omega^{a} \Omega_{b} .
$$

The second result follows from the fact that both sides are simultaneously symmetric in $a$ and $b$, orthogonal to $\Omega_{a}$ and $\Omega^{b}$, and have the same trace.

From the preceding results we establish that the transformation from $\hat{x}^{a}$ to $\left(r, \theta^{A}\right)$ is accomplished by

$$
\frac{\partial \hat{x}^{a}}{\partial r}=\Omega^{a}, \quad \frac{\partial \hat{x}^{a}}{\partial \theta^{A}}=r \Omega_{A}^{a},
$$

while the transformation from $\left(r, \theta^{A}\right)$ to $\hat{x}^{a}$ is accomplished by

$$
\frac{\partial r}{\partial \hat{x}^{a}}=\Omega_{a}, \quad \frac{\partial \theta^{A}}{\partial \hat{x}^{a}}=\frac{1}{r} \Omega_{a}^{A}
$$

With these transformation rules it is easy to show that in the angular coordinates, the metric takes the form of

$$
d s^{2}=g_{u u} d u^{2}+2 g_{u r} d u d r+2 g_{u A} d u d \theta^{A}+g_{A B} d \theta^{A} d \theta^{B}
$$

with

$$
\begin{aligned}
g_{u u} & =-\left(1+r a_{a} \Omega^{a}\right)^{2}+r^{2}\left(a_{a}-\omega_{a b} \Omega^{b}\right)\left(a^{a}-\omega_{c}^{a} \Omega^{c}\right)-r^{2} S+\mathcal{O}\left(r^{3}\right), \\
g_{u r} & =-1 \\
g_{u A} & =r\left[r\left(a_{a}-\omega_{a b} \Omega^{b}\right)+\frac{2}{3} r^{2} S_{a}+\mathcal{O}\left(r^{3}\right)\right] \Omega_{A}^{a}, \\
g_{A B} & =r^{2}\left[\Omega_{A B}-\frac{1}{3} r^{2} S_{a b} \Omega_{A}^{a} \Omega_{B}^{b}+\mathcal{O}\left(r^{3}\right)\right] .
\end{aligned}
$$

The results $g_{r u}=-1, g_{r r}=0$, and $g_{r A}=0$ are exact, and they follow from the fact that in the retarded coordinates, $k_{\alpha} d x^{\alpha}=-d u$ and $k^{\alpha} \partial_{\alpha}=\partial_{r}$.

The nonvanishing components of the inverse metric are

$$
\begin{aligned}
g^{u r} & =-1, \\
g^{r r} & =1+2 r a_{a} \Omega^{a}+r^{2} S+\mathcal{O}\left(r^{3}\right), \\
g^{r A} & =\frac{1}{r}\left[r\left(a^{a}-\omega^{a}{ }_{b} \Omega^{b}\right)+\frac{2}{3} r^{2} S^{a}+\mathcal{O}\left(r^{3}\right)\right] \Omega_{a}^{A}, \\
g^{A B} & =\frac{1}{r^{2}}\left[\Omega^{A B}+\frac{1}{3} r^{2} S^{a b} \Omega_{a}^{A} \Omega_{b}^{B}+\mathcal{O}\left(r^{3}\right)\right] .
\end{aligned}
$$

The results $g^{u u}=0, g^{u r}=-1$, and $g^{u A}=0$ are exact, and they follow from the same reasoning as before.

Finally, we note that in the angular coordinates, the metric determinant is given by

$$
\sqrt{-g}=r^{2} \sqrt{\Omega}\left[1-\frac{1}{6} r^{2}\left(R_{00}+2 R_{0 a} \Omega^{a}+R_{a b} \Omega^{a} \Omega^{b}\right)+\mathcal{O}\left(r^{3}\right)\right]
$$

where $\Omega$ is the determinant of $\Omega_{A B}$; in the canonical parameterization, $\sqrt{\Omega}=\sin \theta$.

Living Reviews in Relativity

http://www . livingreviews . org//rr-2004-6 


\subsubsection{Specialization to $a^{\mu}=0=R_{\mu \nu}$}

In this section we specialize our previous results to a situation where $\gamma$ is a geodesic on which the Ricci tensor vanishes. We therefore set $a^{\mu}=0=R_{\mu \nu}$ everywhere on $\gamma$, and for simplicity we also set $\omega_{a b}$ to zero.

We have seen in Section 3.2.6 that when the Ricci tensor vanishes on $\gamma$, all frame components of the Riemann tensor can be expressed in terms of the symmetric-tracefree tensors $\mathcal{E}_{a b}(u)$ and $\mathcal{B}_{a b}(u)$. The relations are $R_{a 0 b 0}=\mathcal{E}_{a b}, R_{a 0 b c}=\varepsilon_{b c d} \mathcal{B}_{a}^{d}$, and $R_{a c b d}=\delta_{a b} \mathcal{E}_{c d}+\delta_{c d} \mathcal{E}_{a b}-\delta_{a d} \mathcal{E}_{b c}-\delta_{b c} \mathcal{E}_{a d}$. These can be substituted into Equations $(160,161,162)$ to give

$$
\begin{aligned}
S_{a b}\left(u, \theta^{A}\right) & =2 \mathcal{E}_{a b}-\Omega_{a} \mathcal{E}_{b c} \Omega^{c}-\Omega_{b} \mathcal{E}_{a c} \Omega^{c}+\delta_{a b} \mathcal{E}_{b c} \Omega^{c} \Omega^{d}+\varepsilon_{a c d} \Omega^{c} \mathcal{B}_{b}^{d}+\varepsilon_{b c d} \Omega^{c} \mathcal{B}_{a}^{d} \\
S_{a}\left(u, \theta^{A}\right) & =\mathcal{E}_{a b} \Omega^{b}+\varepsilon_{a b c} \Omega^{b} \mathcal{B}^{c}{ }_{d}^{d} \\
S\left(u, \theta^{A}\right) & =\mathcal{E}_{a b} \Omega^{a} \Omega^{b}
\end{aligned}
$$

In these expressions the dependence on retarded time $u$ is contained in $\mathcal{E}_{a b}$ and $\mathcal{B}_{a b}$, while the angular dependence is encoded in the unit vector $\Omega^{a}$.

It is convenient to introduce the irreducible quantities

$$
\begin{aligned}
\mathcal{E}^{*} & =\mathcal{E}_{a b} \Omega^{a} \Omega^{b}, \\
\mathcal{E}_{a}^{*} & =\left(\delta_{a}{ }^{b}-\Omega_{a} \Omega^{b}\right) \mathcal{E}_{b c} \Omega^{c}, \\
\mathcal{E}_{a b}^{*} & =2 \mathcal{E}_{a b}-2 \Omega_{a} \mathcal{E}_{b c} \Omega^{c}-2 \Omega_{b} \mathcal{E}_{a c} \Omega^{c}+\left(\delta_{a b}+\Omega_{a} \Omega_{b}\right) \mathcal{E}^{*}, \\
\mathcal{B}_{a}^{*} & =\varepsilon_{a b c} \Omega^{b} \mathcal{B}^{c}{ }_{d} \Omega^{d}, \\
\mathcal{B}_{a b}^{*} & =\varepsilon_{a c d} \Omega^{c} \mathcal{B}^{d}{ }_{e}\left(\delta^{e}{ }_{b}-\Omega^{e} \Omega_{b}\right)+\varepsilon_{b c d} \Omega^{c} \mathcal{B}^{d}{ }_{e}\left(\delta^{e}{ }_{a}-\Omega^{e} \Omega_{a}\right) .
\end{aligned}
$$

These are all orthogonal to $\Omega^{a}: \mathcal{E}_{a}^{*} \Omega^{a}=\mathcal{B}_{a}^{*} \Omega^{a}=0$ and $\mathcal{E}_{a b}^{*} \Omega^{b}=\mathcal{B}_{a b}^{*} \Omega^{b}=0$. In terms of these Equations $(196,197,198)$ become

$$
\begin{aligned}
S_{a b} & =\mathcal{E}_{a b}^{*}+\Omega_{a} \mathcal{E}_{b}^{*}+\mathcal{E}_{a}^{*} \Omega_{b}+\Omega_{a} \Omega_{b} \mathcal{E}^{*}+\mathcal{B}_{a b}^{*}+\Omega_{a} \mathcal{B}_{b}^{*}+\mathcal{B}_{a}^{*} \Omega_{b}, \\
S_{a} & =\mathcal{E}_{a}^{*}+\Omega_{a} \mathcal{E}^{*}+\mathcal{B}_{a}^{*} \\
S & =\mathcal{E}^{*}
\end{aligned}
$$

When Equations (204, 205, 206) are substituted into the metric tensor of Equations (171, 172, $173)$ - in which $a_{a}$ and $\omega_{a b}$ are both set equal to zero - we obtain the compact expressions

$$
\begin{aligned}
& g_{u u}=-1-r^{2} \mathcal{E}^{*}+\mathcal{O}\left(r^{3}\right), \\
& g_{u a}=-\Omega_{a}+\frac{2}{3} r^{2}\left(\mathcal{E}_{a}^{*}+\mathcal{B}_{a}^{*}\right)+\mathcal{O}\left(r^{3}\right), \\
& g_{a b}=\delta_{a b}-\Omega_{a} \Omega_{b}-\frac{1}{3} r^{2}\left(\mathcal{E}_{a b}^{*}+\mathcal{B}_{a b}^{*}\right)+\mathcal{O}\left(r^{3}\right) .
\end{aligned}
$$

The metric becomes

$$
\begin{aligned}
g_{u u} & =-1-r^{2} \mathcal{E}^{*}+\mathcal{O}\left(r^{3}\right) \\
g_{u r} & =-1 \\
g_{u A} & =\frac{2}{3} r^{3}\left(\mathcal{E}_{A}^{*}+\mathcal{B}_{A}^{*}\right)+\mathcal{O}\left(r^{4}\right) \\
g_{A B} & =r^{2} \Omega_{A B}-\frac{1}{3} r^{4}\left(\mathcal{E}_{A B}^{*}+\mathcal{B}_{A B}^{*}\right)+\mathcal{O}\left(r^{5}\right)
\end{aligned}
$$


after transforming to angular coordinates using the rules of Equation (185). Here we have introduced the projections

$$
\begin{aligned}
\mathcal{E}_{A}^{*} & \equiv \mathcal{E}_{a}^{*} \Omega_{A}^{a}=\mathcal{E}_{a b} \Omega_{A}^{a} \Omega^{b}, \\
\mathcal{E}_{A B}^{*} & \equiv \mathcal{E}_{a b}^{*} \Omega_{A}^{a} \Omega_{B}^{b}=2 \mathcal{E}_{a b} \Omega_{A}^{a} \Omega_{B}^{b}+\mathcal{E}^{*} \Omega_{A B}, \\
\mathcal{B}_{A}^{*} & \equiv \mathcal{B}_{a}^{*} \Omega_{A}^{a}=\varepsilon_{a b c} \Omega_{A}^{a} \Omega^{b} \mathcal{B}^{c}{ }_{d}^{d} \Omega^{d} \\
\mathcal{B}_{A B}^{*} & \equiv \mathcal{B}_{a b}^{*} \Omega_{A}^{a} \Omega_{B}^{b}=2 \varepsilon_{a c d} \Omega^{c} \mathcal{B}^{d}{ }_{b} \Omega_{(A}^{a} \Omega_{B)}^{b} .
\end{aligned}
$$

It may be noted that the inverse relations are $\mathcal{E}_{a}^{*}=\mathcal{E}_{A}^{*} \Omega_{a}^{A}, \mathcal{B}_{a}^{*}=\mathcal{B}_{A}^{*} \Omega_{a}^{A}, \mathcal{E}_{a b}^{*}=\mathcal{E}_{A B}^{*} \Omega_{a}^{A} \Omega_{b}^{B}$, and $\mathcal{B}_{a b}^{*}=\mathcal{B}_{A B}^{*} \Omega_{a}^{A} \Omega_{b}^{B}$, where $\Omega_{a}^{A}$ was introduced in Equation (183).

\subsection{Transformation between Fermi and retarded coordinates; advanced point}

A point $x$ in the normal convex neighbourhood of a world line $\gamma$ can be assigned a set of Fermi normal coordinates (as in Section 3.2), or it can be assigned a set of retarded coordinates (see Section 3.3). These coordinate systems can obviously be related to one another, and our first task in this section (which will occupy us in Sections 3.4.1, 3.4.2, and 3.4.3) will be to derive the transformation rules. We begin by refining our notation so as to eliminate any danger of ambiguity.

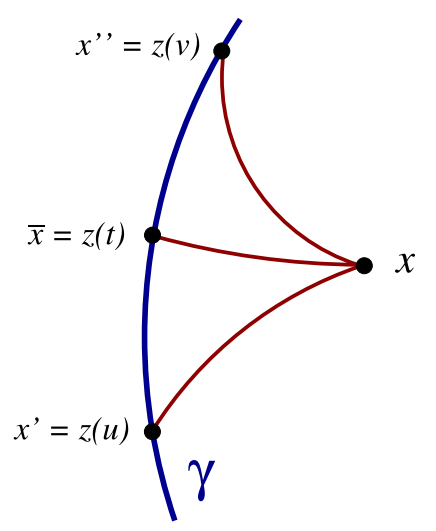

Figure 8: The retarded, simultaneous, and advanced points on a world line $\gamma$. The retarded point $x^{\prime} \equiv z(u)$ is linked to $x$ by a future-directed null geodesic. The simultaneous point $\bar{x} \equiv z(t)$ is linked to $x$ by a spacelike geodesic that intersects $\gamma$ orthogonally. The advanced point $x^{\prime \prime} \equiv z(v)$ is linked to $x$ by a past-directed null geodesic.

The Fermi normal coordinates of $x$ refer to a point $\bar{x} \equiv z(t)$ on $\gamma$ that is related to $x$ by a spacelike geodesic that intersects $\gamma$ orthogonally (see Figure 8). We refer to this point as $x$ 's simultaneous point, and to tensors at $\bar{x}$ we assign indices $\bar{\alpha}, \bar{\beta}$, etc. We let $\left(t, s \omega^{a}\right)$ be the Fermi normal coordinates of $x$, with $t$ denoting the value of $\gamma^{\prime}$ 's proper-time parameter at $\bar{x}, s=\sqrt{2 \sigma(x, \bar{x})}$ representing the proper distance from $\bar{x}$ to $x$ along the spacelike geodesic, and $\omega^{a}$ denoting a unit vector $\left(\delta_{a b} \omega^{a} \omega^{b}=1\right)$ that determines the direction of the geodesic. The Fermi normal coordinates are defined by $s \omega^{a}=-e_{\bar{\alpha}}^{a} \sigma^{\bar{\alpha}}$ and $\sigma_{\bar{\alpha}} u^{\bar{\alpha}}=0$. Finally, we denote by $\left(\bar{e}_{0}^{\alpha}, \bar{e}_{a}^{\alpha}\right)$ the tetrad at $x$ that is obtained by parallel transport of $\left(u^{\bar{\alpha}}, e_{a}^{\bar{\alpha}}\right)$ on the spacelike geodesic.

The retarded coordinates of $x$ refer to a point $x^{\prime} \equiv z(u)$ on $\gamma$ that is linked to $x$ by a futuredirected null geodesic (see Figure 8). We refer to this point as $x$ 's retarded point, and to tensors at $x^{\prime}$ we assign indices $\alpha^{\prime}, \beta^{\prime}$, etc. We let $\left(u, r \Omega^{a}\right)$ be the retarded coordinates of $x$, with $u$ denoting the value of $\gamma^{\prime}$ s proper-time parameter at $x^{\prime}, r=\sigma_{\alpha^{\prime}} u^{\alpha^{\prime}}$ representing the affine-parameter distance from

Living Reviews in Relativity

http://www. livingreviews . org/lrr-2004-6 
$x^{\prime}$ to $x$ along the null geodesic, and $\Omega^{a}$ denoting a unit vector $\left(\delta_{a b} \Omega^{a} \Omega^{b}=1\right)$ that determines the direction of the geodesic. The retarded coordinates are defined by $r \Omega^{a}=-e_{\alpha^{\prime}}^{a} \sigma^{\alpha^{\prime}}$ and $\sigma\left(x, x^{\prime}\right)=0$. Finally, we denote by $\left(e_{0}^{\alpha}, e_{a}^{\alpha}\right)$ the tetrad at $x$ that is obtained by parallel transport of $\left(u^{\alpha^{\prime}}, e_{a}^{\alpha^{\prime}}\right)$ on the null geodesic.

The reader not interested in following the details of this discussion can be informed that

- our results concerning the transformation from the retarded coordinates $\left(u, r, \Omega^{a}\right)$ to the Fermi normal coordinates $\left(t, s, \omega^{a}\right)$ are contained in Equations $(218,219,220)$ below;

- our results concerning the transformation from the Fermi normal coordinates $\left(t, s, \omega^{a}\right)$ to the retarded coordinates $\left(u, r, \Omega^{a}\right)$ are contained in Equations (221, 222, 223);

- the decomposition of each member of $\left(\bar{e}_{0}^{\alpha}, \bar{e}_{a}^{\alpha}\right)$ in the tetrad $\left(e_{0}^{\alpha}, e_{a}^{\alpha}\right)$ is given in retarded coordinates by Equations (224) and (225); and

- the decomposition of each member of $\left(e_{0}^{\alpha}, e_{a}^{\alpha}\right)$ in the tetrad $\left(\bar{e}_{0}^{\alpha}, \bar{e}_{a}^{\alpha}\right)$ is given in Fermi normal coordinates by Equations (226) and (227).

Our final task will be to define, along with the retarded and simultaneous points, an advanced point $x^{\prime \prime}$ on the world line $\gamma$ (see Figure 8). This is taken on in Section 3.4.4. Throughout this section we shall set $\omega_{a b}=0$, where $\omega_{a b}$ is the rotation tensor defined by Equation (138) - the tetrad vectors $e_{a}^{\mu}$ will be assumed to be Fermi-Walker transported on $\gamma$.

\subsubsection{From retarded to Fermi coordinates}

Quantities at $\bar{x} \equiv z(t)$ can be related to quantities at $x^{\prime} \equiv z(u)$ by Taylor expansion along the world line $\gamma$. To implement this strategy we must first find an expression for $\Delta \equiv t-u$. (Although we use the same notation, this should not be confused with the van Vleck determinant introduced in Section 2.5.)

Consider the function $p(\tau)$ of the proper-time parameter $\tau$ defined by

$$
p(\tau)=\sigma_{\mu}(x, z(\tau)) u^{\mu}(\tau),
$$

in which $x$ is kept fixed and in which $z(\tau)$ is an arbitrary point on the world line. We have that $p(u)=r$ and $p(t)=0$, and $\Delta$ can ultimately be obtained by expressing $p(t)$ as $p(u+\Delta)$ and expanding in powers of $\Delta$. Formally,

$$
p(t)=p(u)+\dot{p}(u) \Delta+\frac{1}{2} \ddot{p}(u) \Delta^{2}+\frac{1}{6} p^{(3)}(u) \Delta^{3}+\mathcal{O}\left(\Delta^{4}\right),
$$

where overdots (or a number within brackets) indicate repeated differentiation with respect to $\tau$. We have

$$
\begin{aligned}
\dot{p}(u) & =\sigma_{\alpha^{\prime} \beta^{\prime}} u^{\alpha^{\prime}} u^{\beta^{\prime}}+\sigma_{\alpha^{\prime}} a^{\alpha^{\prime}}, \\
\ddot{p}(u) & =\sigma_{\alpha^{\prime} \beta^{\prime} \gamma^{\prime}} u^{\alpha^{\prime}} u^{\beta^{\prime}} u^{\gamma^{\prime}}+3 \sigma_{\alpha^{\prime} \beta^{\prime}} u^{\alpha^{\prime}} a^{\beta^{\prime}}+\sigma_{\alpha^{\prime}} \dot{a}^{\alpha^{\prime}}, \\
p^{(3)}(u) & =\sigma_{\alpha^{\prime} \beta^{\prime} \gamma^{\prime} \delta} u^{\alpha^{\prime}} u^{\beta^{\prime}} u^{\gamma^{\prime}} u^{\delta^{\prime}}+\sigma_{\alpha^{\prime} \beta^{\prime} \gamma^{\prime}}\left(5 a^{\alpha^{\prime}} u^{\beta^{\prime}} u^{\gamma^{\prime}}+u^{\alpha^{\prime}} u^{\beta^{\prime}} a^{\gamma^{\prime}}\right)+\sigma_{\alpha^{\prime} \beta^{\prime}}\left(3 a^{\alpha^{\prime}} a^{\beta^{\prime}}+4 u^{\alpha^{\prime}} \dot{a}^{\beta^{\prime}}\right)+\sigma_{\alpha^{\prime}} \ddot{a}^{\alpha^{\prime}},
\end{aligned}
$$

where $a^{\mu}=D u^{\mu} / d \tau, \dot{a}^{\mu}=D a^{\mu} / d \tau$, and $\ddot{a}^{\mu}=D \dot{a}^{\mu} / d \tau$.

We now express all of this in retarded coordinates by invoking the expansion of Equation (88) for $\sigma_{\alpha^{\prime} \beta^{\prime}}$ (as well as additional expansions for the higher derivatives of the world function, obtained 
by further differentiation of this result) and the relation $\sigma^{\alpha^{\prime}}=-r\left(u^{\alpha^{\prime}}+\Omega^{a} e_{a}^{\alpha^{\prime}}\right)$ first derived in Equation (144). With a degree of accuracy sufficient for our purposes we obtain

$$
\begin{aligned}
\dot{p}(u) & =-\left[1+r a_{a} \Omega^{a}+\frac{1}{3} r^{2} S+\mathcal{O}\left(r^{3}\right)\right], \\
\ddot{p}(u) & =-r\left(\dot{a}_{0}+\dot{a}_{a} \Omega^{a}\right)+\mathcal{O}\left(r^{2}\right), \\
p^{(3)}(u) & =\dot{a}_{0}+\mathcal{O}(r),
\end{aligned}
$$

where $S=R_{a 0 b 0} \Omega^{a} \Omega^{b}$ was first introduced in Equation (162), and where $\dot{a}_{0} \equiv \dot{a}_{\alpha^{\prime}} u^{\alpha^{\prime}}, \dot{a}_{a} \equiv \dot{a}_{\alpha^{\prime}} e_{a}^{\alpha^{\prime}}$ are the frame components of the covariant derivative of the acceleration vector. To arrive at these results we made use of the identity $a_{\alpha^{\prime}} a^{\alpha^{\prime}}+\dot{a}_{\alpha^{\prime}} u^{\alpha^{\prime}}=0$ that follows from the fact that $a^{\mu}$ is orthogonal to $u^{\mu}$. Notice that there is no distinction between the two possible interpretations $\dot{a}_{a} \equiv d a_{a} / d \tau$ and $\dot{a}_{a} \equiv \dot{a}_{\mu} e_{a}^{\mu}$ for the quantity $\dot{a}_{a}(\tau)$; their equality follows at once from the substitution of $D e_{a}^{\mu} / d \tau=a_{a} u^{\mu}$ (which states that the basis vectors are Fermi-Walker transported on the world line) into the identity $d a_{a} / d \tau=D\left(a_{\nu} e_{a}^{\nu}\right) / d \tau$.

Collecting our results we obtain

$$
r=\left[1+r a_{a} \Omega^{a}+\frac{1}{3} r^{2} S+\mathcal{O}\left(r^{3}\right)\right] \Delta+\frac{1}{2} r\left[\dot{a}_{0}+\dot{a}_{a} \Omega^{a}+\mathcal{O}(r)\right] \Delta^{2}-\frac{1}{6}\left[\dot{a}_{0}+\mathcal{O}(r)\right] \Delta^{3}+\mathcal{O}\left(\Delta^{4}\right),
$$

which can readily be solved for $\Delta \equiv t-u$ expressed as an expansion in powers of $r$. The final result is

$$
t=u+r\left\{1-r a_{a}(u) \Omega^{a}+r^{2}\left[a_{a}(u) \Omega^{a}\right]^{2}-\frac{1}{3} r^{2} \dot{a}_{0}(u)-\frac{1}{2} r^{2} \dot{a}_{a}(u) \Omega^{a}-\frac{1}{3} r^{2} R_{a 0 b 0}(u) \Omega^{a} \Omega^{b}+\mathcal{O}\left(r^{3}\right)\right\},
$$

where we show explicitly that all frame components are evaluated at the retarded point $z(u)$.

To obtain relations between the spatial coordinates we consider the functions

$$
p_{a}(\tau)=-\sigma_{\mu}(x, z(\tau)) e_{a}^{\mu}(\tau)
$$

in which $x$ is fixed and $z(\tau)$ is an arbitrary point on $\gamma$. We have that the retarded coordinates are given by $r \Omega^{a}=p^{a}(u)$, while the Fermi coordinates are given instead by $s \omega^{a}=p^{a}(t)=p^{a}(u+\Delta)$. This last expression can be expanded in powers of $\Delta$, producing

$$
s \omega^{a}=p^{a}(u)+\dot{p}^{a}(u) \Delta+\frac{1}{2} \ddot{p}^{a}(u) \Delta^{2}+\frac{1}{6} p^{a(3)}(u) \Delta^{3}+\mathcal{O}\left(\Delta^{4}\right),
$$

with

$$
\begin{aligned}
\dot{p}_{a}(u)= & -\sigma_{\alpha^{\prime} \beta^{\prime}} e_{a}^{\alpha^{\prime}} u^{\beta^{\prime}}-\left(\sigma_{\alpha^{\prime}} u^{\alpha^{\prime}}\right)\left(a_{\beta^{\prime}} e_{a}^{\beta^{\prime}}\right) \\
= & -r a_{a}-\frac{1}{3} r^{2} S_{a}+\mathcal{O}\left(r^{3}\right) \\
\ddot{p}_{a}(u)= & -\sigma_{\alpha^{\prime} \beta^{\prime} \gamma^{\prime}} e_{a}^{\alpha^{\prime}} u^{\beta^{\prime}} u^{\gamma^{\prime}}-\left(2 \sigma_{\alpha^{\prime} \beta^{\prime}} u^{\alpha^{\prime}} u^{\beta^{\prime}}+\sigma_{\alpha^{\prime}} a^{\alpha^{\prime}}\right)\left(a_{\gamma^{\prime}} e_{a}^{\gamma^{\prime}}\right)-\sigma_{\alpha^{\prime} \beta^{\prime}} e_{a}^{\alpha^{\prime}} a^{\beta^{\prime}}-\left(\sigma_{\alpha^{\prime}} u^{\alpha^{\prime}}\right)\left(\dot{a}_{\beta^{\prime}} e_{a}^{\beta^{\prime}}\right) \\
= & \left(1+r a_{b} \Omega^{b}\right) a_{a}-r \dot{a}_{a}+\frac{1}{3} r R_{a 0 b 0} \Omega^{b}+\mathcal{O}\left(r^{2}\right) \\
p_{a}^{(3)}(u)= & -\sigma_{\alpha^{\prime} \beta^{\prime} \gamma^{\prime} \delta^{\prime}} e_{a}^{\alpha^{\prime}} u^{\beta^{\prime}} u^{\gamma^{\prime}} u^{\delta^{\prime}} \\
& -\left(3 \sigma_{\alpha^{\prime} \beta^{\prime} \gamma^{\prime}} u^{\alpha^{\prime}} u^{\beta^{\prime}} u^{\gamma^{\prime}}+6 \sigma_{\alpha^{\prime} \beta^{\prime}} u^{\alpha^{\prime}} a^{\beta^{\prime}}+\sigma_{\alpha^{\prime}} \dot{a}^{\alpha^{\prime}}+\sigma_{\alpha^{\prime}} u^{\alpha^{\prime}} \dot{a}_{\beta^{\prime}} u^{\beta^{\prime}}\right)\left(a_{\delta^{\prime}} e_{a}^{\delta^{\prime}}\right) \\
& -\sigma_{\alpha^{\prime} \beta^{\prime} \gamma^{\prime}} e_{a}^{\alpha^{\prime}}\left(2 a^{\beta^{\prime}} u^{\gamma^{\prime}}+u^{\beta^{\prime}} a^{\gamma^{\prime}}\right)-\left(3 \sigma_{\alpha^{\prime} \beta^{\prime}} u^{\alpha^{\prime}} u^{\beta^{\prime}}+2 \sigma_{\alpha^{\prime}} a^{\alpha^{\prime}}\right)\left(\dot{a}_{\gamma^{\prime}} e_{a}^{\gamma^{\prime}}\right)-\sigma_{\alpha^{\prime} \beta^{\prime}} e_{a}^{\alpha^{\prime}} \dot{a}^{\beta^{\prime}} \\
& -\left(\sigma_{\alpha^{\prime}} u^{\alpha^{\prime}}\right)\left(\ddot{a}_{\beta^{\prime}} e_{a}^{\beta^{\prime}}\right) \\
= & 2 \dot{a}_{a}+\mathcal{O}(r) .
\end{aligned}
$$

Living Reviews in Relativity

http://www. livingreviews . org/lrr-2004-6 
To arrive at these results we have used the same expansions as before and re-introduced $S_{a}=$ $R_{a 0 b 0} \Omega^{b}-R_{a b 0 c} \Omega^{b} \Omega^{c}$, as it was first defined in Equation (161).

Collecting our results we obtain

$$
\begin{aligned}
s \omega^{a}= & r \Omega^{a}-r\left[a^{a}+\frac{1}{3} r S^{a}+\mathcal{O}\left(r^{2}\right)\right] \Delta+\frac{1}{2}\left[\left(1+r a_{b} \Omega^{b}\right) a^{a}-r \dot{a}^{a}+\frac{1}{3} r R_{0 b 0}^{a} \Omega^{b}+\mathcal{O}\left(r^{2}\right)\right] \Delta^{2} \\
& +\frac{1}{3}\left[\dot{a}^{a}+\mathcal{O}(r)\right] \Delta^{3}+\mathcal{O}\left(\Delta^{4}\right),
\end{aligned}
$$

which becomes

$$
s \omega^{a}=r\left\{\Omega^{a}-\frac{1}{2} r\left[1-r a_{b}(u) \Omega^{b}\right] a^{a}(u)-\frac{1}{6} r^{2} \dot{a}^{a}(u)-\frac{1}{6} r^{2} R_{0 b 0}^{a}(u) \Omega^{b}+\frac{1}{3} r^{2} R_{b 0 c}^{a}(u) \Omega^{b} \Omega^{c}+\mathcal{O}\left(r^{3}\right)\right\}
$$

after substituting Equation (218) for $\Delta \equiv t-u$. From squaring Equation (219) and using the identity $\delta_{a b} \omega^{a} \omega^{b}=1$ we can also deduce

$$
s=r\left\{1-\frac{1}{2} r a_{a}(u) \Omega^{a}+\frac{3}{8} r^{2}\left[a_{a}(u) \Omega^{a}\right]^{2}-\frac{1}{8} r^{2} \dot{a}_{0}(u)-\frac{1}{6} r^{2} \dot{a}_{a}(u) \Omega^{a}-\frac{1}{6} r^{2} R_{a 0 b 0}(u) \Omega^{a} \Omega^{b}+\mathcal{O}\left(r^{3}\right)\right\}
$$

for the spatial distance between $x$ and $z(t)$.

\subsubsection{From Fermi to retarded coordinates}

The techniques developed in the preceding Section 3.4.2 can easily be adapted to the task of relating the retarded coordinates of $x$ to its Fermi normal coordinates. Here we use $\bar{x} \equiv z(t)$ as the reference point and express all quantities at $x^{\prime} \equiv z(u)$ as Taylor expansions about $\tau=t$.

We begin by considering the function

$$
\sigma(\tau)=\sigma(x, z(\tau))
$$

of the proper-time parameter $\tau$ on $\gamma$. We have that $\sigma(t)=\frac{1}{2} s^{2}$ and $\sigma(u)=0$, and $\Delta \equiv t-u$ is now obtained by expressing $\sigma(u)$ as $\sigma(t-\Delta)$ and expanding in powers of $\Delta$. Using the fact that $\dot{\sigma}(\tau)=p(\tau)$, we have

$$
\sigma(u)=\sigma(t)-p(t) \Delta+\frac{1}{2} \dot{p}(t) \Delta^{2}-\frac{1}{6} \ddot{p}(t) \Delta^{3}+\frac{1}{24} p^{(3)}(t) \Delta^{4}+\mathcal{O}\left(\Delta^{5}\right) .
$$

Expressions for the derivatives of $p(\tau)$ evaluated at $\tau=t$ can be constructed from results derived previously in Section 3.4.1: it suffices to replace all primed indices by barred indices and then substitute the relation $\sigma^{\bar{\alpha}}=-s \omega^{a} e_{a}^{\bar{\alpha}}$ that follows immediately from Equation (116). This gives

$$
\begin{aligned}
\dot{p}(t) & =-\left[1+s a_{a} \omega^{a}+\frac{1}{3} s^{2} R_{a 0 b 0} \omega^{a} \omega^{b}+\mathcal{O}\left(s^{3}\right)\right], \\
\ddot{p}(t) & =-s \dot{a}_{a} \omega^{a}+\mathcal{O}\left(s^{2}\right), \\
p^{(3)}(t) & =\dot{a}_{0}+\mathcal{O}(s),
\end{aligned}
$$

and then

$$
s^{2}=\left[1+s a_{a} \omega^{a}+\frac{1}{3} s^{2} R_{a 0 b 0} \omega^{a} \omega^{b}+\mathcal{O}\left(s^{3}\right)\right] \Delta^{2}-\frac{1}{3} s\left[\dot{a}_{a} \omega^{a}+\mathcal{O}(s)\right] \Delta^{3}-\frac{1}{12}\left[\dot{a}_{0}+\mathcal{O}(s)\right] \Delta^{4}+\mathcal{O}\left(\Delta^{5}\right)
$$


after recalling that $p(t)=0$. Solving for $\Delta$ as an expansion in powers of $s$ returns

$$
u=t-s\left\{1-\frac{1}{2} s a_{a}(t) \omega^{a}+\frac{3}{8} s^{2}\left[a_{a}(t) \omega^{a}\right]^{2}+\frac{1}{24} s^{2} \dot{a}_{0}(t)+\frac{1}{6} s^{2} \dot{a}_{a}(t) \omega^{a}-\frac{1}{6} s^{2} R_{a 0 b 0}(t) \omega^{a} \omega^{b}+\mathcal{O}\left(s^{3}\right)\right\}
$$

in which we emphasize that all frame components are evaluated at the simultaneous point $z(t)$.

An expression for $r=p(u)$ can be obtained by expanding $p(t-\Delta)$ in powers of $\Delta$. We have

$$
r=-\dot{p}(t) \Delta+\frac{1}{2} \ddot{p}(t) \Delta^{2}-\frac{1}{6} p^{(3)}(t) \Delta^{3}+\mathcal{O}\left(\Delta^{4}\right),
$$

and substitution of our previous results gives

$$
r=s\left\{1+\frac{1}{2} s a_{a}(t) \omega^{a}-\frac{1}{8} s^{2}\left[a_{a}(t) \omega^{a}\right]^{2}-\frac{1}{8} s^{2} \dot{a}_{0}(t)-\frac{1}{3} s^{2} \dot{a}_{a}(t) \omega^{a}+\frac{1}{6} s^{2} R_{a 0 b 0}(t) \omega^{a} \omega^{b}+\mathcal{O}\left(s^{3}\right)\right\}
$$

for the retarded distance between $x$ and $z(u)$.

Finally, the retarded coordinates $r \Omega^{a}=p^{a}(u)$ can be related to the Fermi coordinates by expanding $p^{a}(t-\Delta)$ in powers of $\Delta$, so that

$$
r \Omega^{a}=p^{a}(t)-\dot{p}^{a}(t) \Delta+\frac{1}{2} \ddot{p}^{a}(t) \Delta^{2}-\frac{1}{6} p^{a(3)}(t) \Delta^{3}+\mathcal{O}\left(\Delta^{4}\right) .
$$

Results from the preceding Section 3.4.2 can again be imported with mild alterations, and we find

$$
\begin{aligned}
\dot{p}_{a}(t) & =\frac{1}{3} s^{2} R_{a b 0 c} \omega^{b} \omega^{c}+\mathcal{O}\left(s^{3}\right), \\
\ddot{p}_{a}(t) & =\left(1+s a_{b} \omega^{b}\right) a_{a}+\frac{1}{3} s R_{a 0 b 0} \omega^{b}+\mathcal{O}\left(s^{2}\right), \\
p_{a}^{(3)}(t) & =2 \dot{a}_{a}(t)+\mathcal{O}(s) .
\end{aligned}
$$

This, together with Equation (221), gives

$$
r \Omega^{a}=s\left\{\omega^{a}+\frac{1}{2} s a^{a}(t)-\frac{1}{3} s^{2} \dot{a}^{a}(t)-\frac{1}{3} s^{2} R_{b 0 c}^{a}(t) \omega^{b} \omega^{c}+\frac{1}{6} s^{2} R_{0 b 0}^{a}(t) \omega^{b}+\mathcal{O}\left(s^{3}\right)\right\} .
$$

It may be checked that squaring this equation and using the identity $\delta_{a b} \Omega^{a} \Omega^{b}=1$ returns the same result as Equation (222).

\subsubsection{Transformation of the tetrads at $x$}

Recall that we have constructed two sets of basis vectors at $x$. The first set is the tetrad $\left(\bar{e}_{0}^{\alpha}, \bar{e}_{a}^{\alpha}\right)$ that is obtained by parallel transport of $\left(u^{\bar{\alpha}}, e_{a}^{\bar{\alpha}}\right)$ on the spacelike geodesic that links $x$ to the simultaneous point $\bar{x} \equiv z(t)$. The second set is the tetrad $\left(e_{0}^{\alpha}, e_{a}^{\alpha}\right)$ that is obtained by parallel transport of $\left(u^{\alpha^{\prime}}, e_{a}^{\alpha^{\prime}}\right)$ on the null geodesic that links $x$ to the retarded point $x^{\prime} \equiv z(u)$. Since each tetrad forms a complete set of basis vectors, each member of $\left(\bar{e}_{0}^{\alpha}, \bar{e}_{a}^{\alpha}\right)$ can be decomposed in the tetrad $\left(e_{0}^{\alpha}, e_{a}^{\alpha}\right)$, and correspondingly, each member of $\left(e_{0}^{\alpha}, e_{a}^{\alpha}\right)$ can be decomposed in the tetrad $\left(\bar{e}_{0}^{\alpha}, \bar{e}_{a}^{\alpha}\right)$. These decompositions are worked out in this Section. For this purpose we shall consider the functions

$$
p^{\alpha}(\tau)=g_{\mu}^{\alpha}(x, z(\tau)) u^{\mu}(\tau), \quad p_{a}^{\alpha}(\tau)=g_{\mu}^{\alpha}(x, z(\tau)) e_{a}^{\mu}(\tau)
$$

Living Reviews in Relativity

http://www.livingreviews.org/lrr-2004-6 
in which $x$ is a fixed point in a neighbourhood of $\gamma, z(\tau)$ is an arbitrary point on the world line, and $g_{\mu}^{\alpha}(x, z)$ is the parallel propagator on the unique geodesic that links $x$ to $z$. We have $\bar{e}_{0}^{\alpha}=p^{\alpha}(t)$, $\bar{e}_{a}^{\alpha}=p_{a}^{\alpha}(t), e_{0}^{\alpha}=p^{\alpha}(u)$, and $e_{a}^{\alpha}=p_{a}^{\alpha}(u)$.

We begin with the decomposition of $\left(\bar{e}_{0}^{\alpha}, \bar{e}_{a}^{\alpha}\right)$ in the tetrad $\left(e_{0}^{\alpha}, e_{a}^{\alpha}\right)$ associated with the retarded point $z(u)$. This decomposition will be expressed in the retarded coordinates as an expansion in powers of $r$. As in Section 3.2.1 we express quantities at $z(t)$ in terms of quantities at $z(u)$ by expanding in powers of $\Delta \equiv t-u$. We have

$$
\bar{e}_{0}^{\alpha}=p^{\alpha}(u)+\dot{p}^{\alpha}(u) \Delta+\frac{1}{2} \ddot{p}^{\alpha}(u) \Delta^{2}+\mathcal{O}\left(\Delta^{3}\right),
$$

with

$$
\begin{aligned}
\dot{p}^{\alpha}(u) & =g_{\alpha^{\prime} ; \beta^{\prime}}^{\alpha} u^{\alpha^{\prime}} u^{\beta^{\prime}}+g_{\alpha^{\prime}}^{\alpha} a^{\alpha^{\prime}} \\
& =\left[a^{a}+\frac{1}{2} r R_{0 b 0}^{a} \Omega^{b}+\mathcal{O}\left(r^{2}\right)\right] e_{a}^{\alpha}, \\
\ddot{p}^{\alpha}(u) & =g_{\alpha^{\prime} ; \beta^{\prime} \gamma^{\prime}}^{\alpha} u^{\alpha^{\prime}} u^{\beta^{\prime}} u^{\gamma^{\prime}}+g^{\alpha}{ }_{\alpha^{\prime} ; \beta^{\prime}}\left(2 a^{\alpha^{\prime}} u^{\beta^{\prime}}+u^{\alpha^{\prime}} a^{\beta^{\prime}}\right)+g_{\alpha^{\prime}}^{\alpha} \dot{a}^{\alpha^{\prime}} \\
& =\left[-\dot{a}_{0}+\mathcal{O}(r)\right] e_{0}^{\alpha}+\left[\dot{a}^{a}+\mathcal{O}(r)\right] e_{a}^{\alpha},
\end{aligned}
$$

where we have used the expansions of Equation (92) as well as the decompositions of Equation (141). Collecting these results and substituting Equation (218) for $\Delta$ yields

$$
\bar{e}_{0}^{\alpha}=\left[1-\frac{1}{2} r^{2} \dot{a}_{0}(u)+\mathcal{O}\left(r^{3}\right)\right] e_{0}^{\alpha}+\left[r\left(1-a_{b} \Omega^{b}\right) a^{a}(u)+\frac{1}{2} r^{2} \dot{a}^{a}(u)+\frac{1}{2} r^{2} R_{0 b 0}^{a}(u) \Omega^{b}+\mathcal{O}\left(r^{3}\right)\right] e_{a}^{\alpha} .
$$

Similarly, we have

$$
\bar{e}_{a}^{\alpha}=p_{a}^{\alpha}(u)+\dot{p}_{a}^{\alpha}(u) \Delta+\frac{1}{2} \ddot{p}_{a}^{\alpha}(u) \Delta^{2}+\mathcal{O}\left(\Delta^{3}\right)
$$

with

$$
\begin{aligned}
\dot{p}_{a}^{\alpha}(u) & =g_{\alpha^{\prime} ; \beta^{\prime}}^{\alpha} e_{a}^{\alpha^{\prime}} u^{\beta^{\prime}}+\left(g_{\alpha^{\prime}}^{\alpha} u^{\alpha^{\prime}}\right)\left(a_{\beta^{\prime}} e_{a}^{\beta^{\prime}}\right) \\
& =\left[a_{a}+\frac{1}{2} r R_{a 0 b 0} \Omega^{b}+\mathcal{O}\left(r^{2}\right)\right] e_{0}^{\alpha}+\left[-\frac{1}{2} r R_{a 0 c}^{b} \Omega^{c}+\mathcal{O}\left(r^{2}\right)\right] e_{b}^{\alpha}, \\
\ddot{p}_{a}^{\alpha}(u) & =g_{\alpha^{\prime} ; \beta^{\prime} \gamma^{\prime}}^{\alpha} e_{a}^{\alpha^{\prime}} u^{\beta^{\prime}} u^{\gamma^{\prime}}+g_{\alpha^{\prime} ; \beta^{\prime}}^{\alpha}\left(2 u^{\alpha^{\prime}} u^{\beta^{\prime}} a_{\gamma^{\prime}} e_{a}^{\gamma^{\prime}}+e_{a}^{\alpha^{\prime}} a^{\beta^{\prime}}\right)+\left(g^{\alpha}{ }_{\alpha^{\prime}} a^{\alpha^{\prime}}\right)\left(a_{\beta^{\prime}} e_{a}^{\beta^{\prime}}\right)+\left(g_{\alpha^{\prime}}^{\alpha} u^{\alpha^{\prime}}\right)\left(\dot{a}_{\beta^{\prime}} e_{a}^{\beta^{\prime}}\right) \\
& =\left[\dot{a}_{a}+\mathcal{O}(r)\right] e_{0}^{\alpha}+\left[a_{a} a^{b}+\mathcal{O}(r)\right] e_{b}^{\alpha},
\end{aligned}
$$

and all this gives

$$
\begin{aligned}
\bar{e}_{a}^{\alpha}= & {\left[\delta^{b}{ }_{a}+\frac{1}{2} r^{2} a^{b}(u) a_{a}(u)-\frac{1}{2} r^{2} R_{a 0 c}^{b}(u) \Omega^{c}+\mathcal{O}\left(r^{3}\right)\right] e_{b}^{\alpha} } \\
& +\left[r\left(1-r a_{b} \Omega^{b}\right) a_{a}(u)+\frac{1}{2} r^{2} \dot{a}_{a}(u)+\frac{1}{2} r^{2} R_{a 0 b 0}(u) \Omega^{b}+\mathcal{O}\left(r^{3}\right)\right] e_{0}^{\alpha} .
\end{aligned}
$$

We now turn to the decomposition of $\left(e_{0}^{\alpha}, e_{a}^{\alpha}\right)$ in the tetrad $\left(\bar{e}_{0}^{\alpha}, \bar{e}_{a}^{\alpha}\right)$ associated with the simultaneous point $z(t)$. This decomposition will be expressed in the Fermi normal coordinates as an expansion in powers of $s$. Here, as in Section 3.2.2, we shall express quantities at $z(u)$ in terms of quantities at $z(t)$. We begin with

$$
e_{0}^{\alpha}=p^{\alpha}(t)-\dot{p}^{\alpha}(t) \Delta+\frac{1}{2} \ddot{p}^{\alpha}(t) \Delta^{2}+\mathcal{O}\left(\Delta^{3}\right),
$$


and we evaluate the derivatives of $p^{\alpha}(\tau)$ at $\tau=t$. To accomplish this we rely on our previous results (replacing primed indices with barred indices), on the expansions of Equation (92), and on the decomposition of $g_{\bar{\alpha}}^{\alpha}(x, \bar{x})$ in the tetrads at $x$ and $\bar{x}$. This gives

$$
\begin{aligned}
& \dot{p}^{\alpha}(t)=\left[a^{a}+\frac{1}{2} s R_{0 b 0}^{a} \omega^{b}+\mathcal{O}\left(s^{2}\right)\right] \bar{e}_{a}^{\alpha}, \\
& \ddot{p}^{\alpha}(t)=\left[-\dot{a}_{0}+\mathcal{O}(s)\right] \bar{e}_{0}^{\alpha}+\left[\dot{a}^{a}+\mathcal{O}(s)\right] \bar{e}_{a}^{\alpha},
\end{aligned}
$$

and we finally obtain

$$
e_{0}^{\alpha}=\left[1-\frac{1}{2} s^{2} \dot{a}_{0}(t)+\mathcal{O}\left(s^{3}\right)\right] \bar{e}_{0}^{\alpha}+\left[-s\left(1-\frac{1}{2} s a_{b} \omega^{b}\right) a^{a}(t)+\frac{1}{2} s^{2} \dot{a}^{a}(t)-\frac{1}{2} s^{2} R_{0 b 0}^{a}(t) \omega^{b}+\mathcal{O}\left(s^{3}\right)\right] \bar{e}_{a}^{\alpha} .
$$

Similarly, we write

$$
e_{a}^{\alpha}=p_{a}^{\alpha}(t)-\dot{p}_{a}^{\alpha}(t) \Delta+\frac{1}{2} \ddot{p}_{a}^{\alpha}(t) \Delta^{2}+\mathcal{O}\left(\Delta^{3}\right),
$$

in which we substitute

$$
\begin{aligned}
& \dot{p}_{a}^{\alpha}(t)=\left[a_{a}+\frac{1}{2} s R_{a 0 b 0} \omega^{b}+\mathcal{O}\left(s^{2}\right)\right] \bar{e}_{0}^{\alpha}+\left[-\frac{1}{2} s R_{a 0 c}^{b} \omega^{c}+\mathcal{O}\left(s^{2}\right)\right] \bar{e}_{b}^{\alpha}, \\
& \ddot{p}_{a}^{\alpha}(t)=\left[\dot{a}_{a}+\mathcal{O}(s)\right] \bar{e}_{0}^{\alpha}+\left[a_{a} a^{b}+\mathcal{O}(s)\right] \bar{e}_{b}^{\alpha},
\end{aligned}
$$

as well as Equation (221) for $\Delta \equiv t-u$. Our final result is

$$
\begin{aligned}
e_{a}^{\alpha}= & {\left[\delta^{b}{ }_{a}+\frac{1}{2} s^{2} a^{b}(t) a_{a}(t)+\frac{1}{2} s^{2} R_{a 0 c}^{b}(t) \omega^{c}+\mathcal{O}\left(s^{3}\right)\right] \bar{e}_{b}^{\alpha} } \\
& +\left[-s\left(1-\frac{1}{2} s a_{b} \omega^{b}\right) a_{a}(t)+\frac{1}{2} s^{2} \dot{a}_{a}(t)-\frac{1}{2} s^{2} R_{a 0 b 0}(u) \omega^{b}+\mathcal{O}\left(s^{3}\right)\right] \bar{e}_{0}^{\alpha} .
\end{aligned}
$$

\subsubsection{Advanced point}

It will prove convenient to introduce on the world line, along with the retarded and simultaneous points, an advanced point associated with the field point $x$. The advanced point will be denoted $x^{\prime \prime} \equiv z(v)$, with $v$ denoting the value of the proper-time parameter at $x^{\prime \prime}$; to tensors at this point we assign indices $\alpha^{\prime \prime}, \beta^{\prime \prime}$, etc. The advanced point is linked to $x$ by a past-directed null geodesic (refer back to Figure 8), and it can be located by solving $\sigma\left(x, x^{\prime \prime}\right)=0$ together with the requirement that $\sigma^{\alpha^{\prime \prime}}\left(x, x^{\prime \prime}\right)$ be a future-directed null vector. The affine-parameter distance between $x$ and $x^{\prime \prime}$ along the null geodesic is given by

$$
r_{\mathrm{adv}}=-\sigma_{\alpha^{\prime \prime}} u^{\alpha^{\prime \prime}}
$$

and we shall call this the advanced distance between $x$ and the world line. Notice that $r_{\text {adv }}$ is a positive quantity.

We wish first to find an expression for $v$ in terms of the retarded coordinates of $x$. For this purpose we define $\Delta^{\prime} \equiv v-u$ and re-introduce the function $\sigma(\tau) \equiv \sigma(x, z(\tau))$ first considered in Section 3.4.2. We have that $\sigma(v)=\sigma(u)=0$, and $\Delta^{\prime}$ can ultimately be obtained by expressing $\sigma(v)$ as $\sigma\left(u+\Delta^{\prime}\right)$ and expanding in powers of $\Delta^{\prime}$. Recalling that $\dot{\sigma}(\tau)=p(\tau)$, we have

$$
\sigma(v)=\sigma(u)+p(u) \Delta^{\prime}+\frac{1}{2} \dot{p}(u) \Delta^{\prime 2}+\frac{1}{6} \ddot{p}(u) \Delta^{\prime 3}+\frac{1}{24} p^{(3)}(u) \Delta^{\prime 4}+\mathcal{O}\left(\Delta^{\prime 5}\right)
$$

Living Reviews in Relativity

http: //www . livingreviews . org/lrr-2004-6 
Using the expressions for the derivatives of $p(\tau)$ that were first obtained in Section 3.4.1, we write this as

$r=\frac{1}{2}\left[1+r a_{a} \Omega^{a}+\frac{1}{3} r^{2} S+\mathcal{O}\left(r^{3}\right)\right] \Delta^{\prime}+\frac{1}{6} r\left[\dot{a}_{0}+\dot{a}_{a} \Omega^{a}+\mathcal{O}(r)\right] \Delta^{\prime 2}-\frac{1}{24}\left[\dot{a}_{0}+\mathcal{O}(r)\right] \Delta^{\prime 3}+\mathcal{O}\left(\Delta^{\prime 4}\right)$.

Solving for $\Delta^{\prime}$ as an expansion in powers of $r$, we obtain

$$
v=u+2 r\left\{1-r a_{a}(u) \Omega^{a}+r^{2}\left[a_{a}(u) \Omega^{a}\right]^{2}-\frac{1}{3} r^{2} \dot{a}_{0}(u)-\frac{2}{3} r^{2} \dot{a}_{a}(u) \Omega^{a}-\frac{1}{3} r^{2} R_{a 0 b 0}(u) \Omega^{a} \Omega^{b}+\mathcal{O}\left(r^{3}\right)\right\},
$$

in which all frame components are evaluated at the retarded point $z(u)$.

Our next task is to derive an expression for the advanced distance $r_{\text {adv }}$. For this purpose we observe that $r_{\text {adv }}=-p(v)=-p\left(u+\Delta^{\prime}\right)$, which we can expand in powers of $\Delta^{\prime} \equiv v-u$. This gives

$$
r_{\mathrm{adv}}=-p(u)-\dot{p}(u) \Delta^{\prime}-\frac{1}{2} \ddot{p}(u) \Delta^{\prime 2}-\frac{1}{6} p^{(3)}(u) \Delta^{\prime 3}+\mathcal{O}\left(\Delta^{\prime 4}\right),
$$

which then becomes

$r_{\mathrm{adv}}=-r+\left[1+r a_{a} \Omega^{a}+\frac{1}{3} r^{2} S+\mathcal{O}\left(r^{3}\right)\right] \Delta^{\prime}+\frac{1}{2} r\left[\dot{a}_{0}+\dot{a}_{a} \Omega^{a}+\mathcal{O}(r)\right] \Delta^{\prime 2}-\frac{1}{6}\left[\dot{a}_{0}+\mathcal{O}(r)\right] \Delta^{\prime 3}+\mathcal{O}\left(\Delta^{\prime 4}\right)$.

After substituting Equation (229) for $\Delta^{\prime}$ and witnessing a number of cancellations, we arrive at the simple expression

$$
r_{\mathrm{adv}}=r\left[1+\frac{2}{3} r^{2} \dot{a}_{a}(u) \Omega^{a}+\mathcal{O}\left(r^{3}\right)\right] .
$$

From Equations (166), (167), and (229) we deduce that the gradient of the advanced time $v$ is given by

$$
\partial_{\alpha} v=\left[1-2 r a_{a} \Omega^{a}+\mathcal{O}\left(r^{2}\right)\right] e_{\alpha}^{0}+\left[\Omega_{a}-2 r a_{a}+\mathcal{O}\left(r^{2}\right)\right] e_{\alpha}^{a},
$$

where the expansion in powers of $r$ was truncated to a sufficient number of terms. Similarly, Equations $(167,168,230)$ imply that the gradient of the advanced distance is given by

$$
\begin{aligned}
\partial_{\alpha} r_{\mathrm{adv}}= & {\left[\left(1+r a_{b} \Omega^{b}+\frac{4}{3} r^{2} \dot{a}_{b} \Omega^{b}+\frac{1}{3} r^{2} S\right) \Omega_{a}+\frac{2}{3} r^{2} \dot{a}_{a}+\frac{1}{6} r^{2} S_{a}+\mathcal{O}\left(r^{3}\right)\right] e_{\alpha}^{a} } \\
& +\left[-r a_{a} \Omega^{a}-\frac{1}{2} r^{2} S+\mathcal{O}\left(r^{3}\right)\right] e_{\alpha}^{0},
\end{aligned}
$$

where $S_{a}$ and $S$ were first introduced in Equations (161) and (162), respectively. We emphasize that in Equations (231) and (232), all frame components are evaluated at the retarded point $z(u)$. 


\section{Green's Functions}

\subsection{Scalar Green's functions in flat spacetime}

\subsubsection{Green's equation for a massive scalar field}

To prepare the way for our discussion of Green's functions in curved spacetime, we consider first the slightly nontrivial case of a massive scalar field $\Phi(x)$ in flat spacetime. This field satisfies the wave equation

$$
\left(\square-k^{2}\right) \Phi(x)=-4 \pi \mu(x),
$$

where $\square=\eta^{\alpha \beta} \partial_{\alpha} \partial_{\beta}$ is the wave operator, $\mu(x)$ a prescribed source, and where the mass parameter $k$ has a dimension of inverse length. We seek a Green's function $G\left(x, x^{\prime}\right)$ such that a solution to Equation (233) can be expressed as

$$
\Phi(x)=\int G\left(x, x^{\prime}\right) \mu\left(x^{\prime}\right) d^{4} x^{\prime}
$$

where the integration is over all of Minkowski spacetime. The relevant wave equation for the Green's function is

$$
\left(\square-k^{2}\right) G\left(x, x^{\prime}\right)=-4 \pi \delta_{4}\left(x-x^{\prime}\right),
$$

where $\delta_{4}\left(x-x^{\prime}\right)=\delta\left(t-t^{\prime}\right) \delta\left(x-x^{\prime}\right) \delta\left(y-y^{\prime}\right) \delta\left(z-z^{\prime}\right)$ is a four-dimensional Dirac distribution in flat spacetime. Two types of Green's functions will be of particular interest: the retarded Green's function, a solution to Equation (235) with the property that it vanishes if $x$ is in the past of $x^{\prime}$, and the advanced Green's function, which vanishes when $x$ is in the future of $x^{\prime}$.

To solve Equation (235) we use Lorentz invariance and the fact that the spacetime is homogeneous to argue that the retarded and advanced Green's functions must be given by expressions of the form

$$
G_{\text {ret }}\left(x, x^{\prime}\right)=\theta\left(t-t^{\prime}\right) g(\sigma), \quad G_{\mathrm{adv}}\left(x, x^{\prime}\right)=\theta\left(t^{\prime}-t\right) g(\sigma),
$$

where $\sigma=\frac{1}{2} \eta_{\alpha \beta}\left(x-x^{\prime}\right)^{\alpha}\left(x-x^{\prime}\right)^{\beta}$ is Synge's world function in flat spacetime, and where $g(\sigma)$ is a function to be determined. For the remainder of this section we set $x^{\prime}=0$ without loss of generality.

\subsubsection{Integration over the source}

The Dirac functional on the right-hand side of Equation (235) is a highly singular quantity, and we can avoid dealing with it by integrating the equation over a small four-volume $V$ that contains $x^{\prime} \equiv 0$. This volume is bounded by a closed hypersurface $\partial V$. After using Gauss' theorem on the first term of Equation (235), we obtain $\oint_{\partial V} G^{; \alpha} d \Sigma_{\alpha}-k^{2} \int_{V} G d V=-4 \pi$, where $d \Sigma_{\alpha}$ is a surface element on $\partial V$. Assuming that the integral of $G$ over $V$ goes to zero in the limit $V \rightarrow 0$, we have

$$
\lim _{V \rightarrow 0} \oint_{\partial V} G^{; \alpha} d \Sigma_{\alpha}=-4 \pi
$$

It should be emphasized that the four-volume $V$ must contain the point $x^{\prime}$.

To examine Equation (237) we introduce coordinates $(w, \chi, \theta, \phi)$ defined by

$$
t=w \cos \chi, \quad x=w \sin \chi \sin \theta \cos \phi, \quad y=w \sin \chi \sin \theta \sin \phi, \quad z=w \sin \chi \cos \theta,
$$

and we let $\partial V$ be a surface of constant $w$. The metric of flat spacetime is given by

$$
d s^{2}=-\cos 2 \chi d w^{2}+2 w \sin 2 \chi d w d \chi+w^{2} \cos 2 \chi d \chi^{2}+w^{2} \sin ^{2} \chi d \Omega^{2}
$$

Living Reviews in Relativity

http: //www . livingreviews . org//rr-2004-6 
in the new coordinates, where $d \Omega^{2}=d \theta^{2}+\sin ^{2} \theta d \phi^{2}$. Notice that $w$ is a timelike coordinate when $\cos 2 \chi>0$, and that $\chi$ is then a spacelike coordinate; the roles are reversed when $\cos 2 \chi<$ 0 . Straightforward computations reveal that in these coordinates $\sigma=-\frac{1}{2} w^{2} \cos 2 \chi, \sqrt{-g}=$ $w^{3} \sin ^{2} \chi \sin \theta, g^{w w}=-\cos 2 \chi, g^{w \chi}=w^{-1} \sin 2 \chi, g^{\chi \chi}=w^{-2} \cos 2 \chi$, and the only nonvanishing component of the surface element is $d \Sigma_{w}=w^{3} \sin ^{2} \chi d \chi d \Omega$, where $d \Omega=\sin \theta d \theta d \phi$. To calculate the gradient of the Green's function we express it as $G=\theta( \pm t) g(\sigma)=\theta( \pm w \cos \chi) g\left(-\frac{1}{2} w^{2} \cos 2 \chi\right)$, with the upper (lower) sign belonging to the retarded (advanced) Green's function. Calculation gives $G^{; \alpha} d \Sigma_{\alpha}=\theta( \pm \cos \chi) w^{4} \sin ^{2} \chi g^{\prime}(\sigma) d \chi d \Omega$, with a prime indicating differentiation with respect to $\sigma$; it should be noted that derivatives of the step function do not appear in this expression.

Integration of $G^{; \alpha} d \Sigma_{\alpha}$ with respect to $d \Omega$ is immediate, and we find that Equation (237) reduces to

$$
\lim _{w \rightarrow 0} \int_{0}^{\pi} \theta( \pm \cos \chi) w^{4} \sin ^{2} \chi g^{\prime}(\sigma) d \chi=-1 .
$$

For the retarded Green's function, the step function restricts the domain of integration to $0<\chi<$ $\pi / 2$, in which $\sigma$ increases from $-\frac{1}{2} w^{2}$ to $\frac{1}{2} w^{2}$. Changing the variable of integration from $\chi$ to $\sigma$ transforms Equation (238) into

$$
\lim _{\epsilon \rightarrow 0} \epsilon \int_{-\epsilon}^{\epsilon} w(\sigma / \epsilon) g^{\prime}(\sigma) d \sigma=-1, \quad w(\xi) \equiv \sqrt{\frac{1+\xi}{1-\xi}},
$$

where $\epsilon \equiv \frac{1}{2} w^{2}$. For the advanced Green's function, the domain of integration is $\pi / 2<\chi<\pi$, in which $\sigma$ decreases from $\frac{1}{2} w^{2}$ to $-\frac{1}{2} w^{2}$. Changing the variable of integration from $\chi$ to $\sigma$ also produces Equation (239).

\subsubsection{Singular part of $g(\sigma)$}

We have seen that Equation (239) properly encodes the influence of the singular source term on both the retarded and advanced Green's function. The function $g(\sigma)$ that enters into the expressions of Equation (236) must therefore be such that Equation (239) is satisfied. It follows immediately that $g(\sigma)$ must be a singular function, because for a smooth function the integral of Equation (239) would be of order $\epsilon$, and the left-hand side of Equation (239) could never be made equal to -1 . The singularity, however, must be integrable, and this leads us to assume that $g^{\prime}(\sigma)$ must be made out of Dirac $\delta$-functions and derivatives.

We make the ansatz

$$
g(\sigma)=V(\sigma) \theta(-\sigma)+A \delta(\sigma)+B \delta^{\prime}(\sigma)+C \delta^{\prime \prime}(\sigma)+\ldots,
$$

where $V(\sigma)$ is a smooth function, and $A, B, C, \ldots$ are constants. The first term represents a function supported within the past and future light cones of $x^{\prime} \equiv 0$; we exclude a term proportional to $\theta(\sigma)$ for reasons of causality. The other terms are supported on the past and future light cones. It is sufficient to take the coefficients in front of the $\delta$-functions to be constants. To see this we invoke the distributional identities

$$
\sigma \delta(\sigma)=0 \quad \rightarrow \quad \sigma \delta^{\prime}(\sigma)+\delta(\sigma)=0 \quad \rightarrow \quad \sigma \delta^{\prime \prime}(\sigma)+2 \delta^{\prime}(\sigma)=0 \quad \rightarrow \quad \ldots
$$

from which it follows that $\sigma^{2} \delta^{\prime}(\sigma)=\sigma^{3} \delta^{\prime \prime}(\sigma)=\cdots=0$. A term like $f(\sigma) \delta(\sigma)$ is then distributionally equal to $f(0) \delta(\sigma)$, while a term like $f(\sigma) \delta^{\prime}(\sigma)$ is distributionally equal to $f(0) \delta^{\prime}(\sigma)-f^{\prime}(0) \delta(\sigma)$, and a term like $f(\sigma) \delta^{\prime \prime}(\sigma)$ is distributionally equal to $f(0) \delta^{\prime \prime}(\sigma)-2 f^{\prime}(0) \delta^{\prime}(\sigma)+2 f^{\prime \prime}(0) \delta(\sigma)$; here $f(\sigma)$ is an arbitrary test function. Summing over such terms, we recover an expression of the form of Equation (241), and there is no need to make $A, B, C, \ldots$ functions of $\sigma$. 
Differentiation of Equation (240) and substitution into Equation (239) yields

$$
\epsilon \int_{-\epsilon}^{\epsilon} w(\sigma / \epsilon) g^{\prime}(\sigma) d \sigma=\epsilon\left[\int_{-\epsilon}^{\epsilon} V^{\prime}(\sigma) w(\sigma / \epsilon) d \sigma-V(0) w(0)-\frac{A}{\epsilon} \dot{w}(0)+\frac{B}{\epsilon^{2}} \ddot{w}(0)-\frac{C}{\epsilon^{3}} w^{(3)}(0)+\ldots\right]
$$

where overdots (or a number within brackets) indicate repeated differentiation with respect to $\xi \equiv \sigma / \epsilon$. The limit $\epsilon \rightarrow 0$ exists if and only if $B=C=\cdots=0$. In the limit we must then have $A \dot{w}(0)=1$, which implies $A=1$. We conclude that $g(\sigma)$ must have the form of

$$
g(\sigma)=\delta(\sigma)+V(\sigma) \theta(-\sigma)
$$

with $V(\sigma)$ being a smooth function that cannot be determined from Equation (239) alone.

\subsubsection{Smooth part of $g(\sigma)$}

To determine $V(\sigma)$ we must go back to the differential equation of Equation (235). Because the singular structure of the Green's function is now under control, we can safely set $x \neq x^{\prime} \equiv 0$ in the forthcoming operations. This means that the equation to solve is in fact $\left(\square-k^{2}\right) g(\sigma)=0$, the homogeneous version of Equation (235). We have $\nabla_{\alpha} g=g^{\prime} \sigma_{\alpha}, \nabla_{\alpha} \nabla_{\beta} g=g^{\prime \prime} \sigma_{\alpha} \sigma_{\beta}+g^{\prime} \sigma_{\alpha \beta}$, and $\square g=2 \sigma g^{\prime \prime}+4 g^{\prime}$, so that Green's equation reduces to the ordinary differential equation

$$
2 \sigma g^{\prime \prime}+4 g^{\prime}-k^{2} g=0 .
$$

If we substitute Equation (242) into this we get

$$
-\left(2 V+k^{2}\right) \delta(\sigma)+\left(2 \sigma V^{\prime \prime}+4 V^{\prime}-k^{2} V\right) \theta(-\sigma)=0,
$$

where we have used the identities of Equation (241). The left-hand side will vanish as a distribution if we set

$$
2 \sigma V^{\prime \prime}+4 V^{\prime}-k^{2} V=0, \quad V(0)=-\frac{1}{2} k^{2} .
$$

These equations determine $V(\sigma)$ uniquely, even in the absence of a second boundary condition at $\sigma=0$, because the differential equation is singular at $\sigma=0$ and $V$ is known to be smooth.

To solve Equation (244) we let $V=F(z) / z$, with $z \equiv k \sqrt{-2 \sigma}$. This gives rise to Bessel's equation for the new function $F$ :

$$
z^{2} F_{z z}+z F_{z}+\left(z^{2}-1\right) F=0 .
$$

The solution that is well behaved near $z=0$ is $F=a J_{1}(z)$, where $a$ is a constant to be determined. We have that $J_{1}(z) \sim \frac{1}{2} z$ for small values of $z$, and it follows that $V \sim a / 2$. From Equation (244) we see that $a=-k^{2}$. So we have found that the only acceptable solution to Equation (244) is

$$
V(\sigma)=-\frac{k}{\sqrt{-2 \sigma}} J_{1}(k \sqrt{-2 \sigma}) \text {. }
$$

To summarize, the retarded and advanced solutions to Equation (235) are given by Equation (236) with $g(\sigma)$ given by Equation (242) and $V(\sigma)$ given by Equation (245).

\subsubsection{Advanced distributional methods}

The techniques developed previously to find Green's functions for the scalar wave equation are limited to flat spacetime, and they would not be very useful for curved spacetimes. To pursue this generalization we must introduce more powerful distributional methods. We do so in this Section, and in the next we shall use them to recover our previous results.

Living Reviews in Relativity

http: //www . livingreviews . org/lrr-2004-6 
Let $\theta_{+}(x, \Sigma)$ be a generalized step function, defined to be one if $x$ is in the future of the spacelike hypersurface $\Sigma$, and defined to be zero otherwise. Similarly, define $\theta_{-}(x, \Sigma) \equiv 1-\theta_{+}(x, \Sigma)$ to be one if $x$ is in the past of the spacelike hypersurface $\Sigma$, and zero otherwise. Then define the light-cone step functions

$$
\theta_{ \pm}(-\sigma)=\theta_{ \pm}(x, \Sigma) \theta(-\sigma), \quad x^{\prime} \in \Sigma,
$$

so that $\theta_{+}(-\sigma)$ is one if $x$ is an element of $I^{+}\left(x^{\prime}\right)$, the chronological future of $x^{\prime}$, and zero otherwise, and $\theta_{-}(-\sigma)$ is one if $x$ is an element of $I^{-}\left(x^{\prime}\right)$, the chronological past of $x^{\prime}$, and zero otherwise; the choice of hypersurface is immaterial so long as $\Sigma$ is spacelike and contains the reference point $x^{\prime}$. Notice that $\theta_{+}(-\sigma)+\theta_{-}(-\sigma)=\theta(-\sigma)$. Define also the light-cone Dirac functionals

$$
\delta_{ \pm}(\sigma)=\theta_{ \pm}(x, \Sigma) \delta(\sigma), \quad x^{\prime} \in \Sigma,
$$

so that $\delta_{+}(\sigma)$, when viewed as a function of $x$, is supported on the future light cone of $x^{\prime}$, while $\delta_{-}(\sigma)$ is supported on its past light cone. Notice that $\delta_{+}(\sigma)+\delta_{-}(\sigma)=\delta(\sigma)$. In Equations (246) and (247), $\sigma$ is the world function for flat spacetime; it is negative if $x$ and $x^{\prime}$ are timelike related, and positive if they are spacelike related.

The distributions $\theta_{ \pm}(-\sigma)$ and $\delta_{ \pm}(\sigma)$ are not defined at $x=x^{\prime}$ and they cannot be differentiated there. This pathology can be avoided if we shift $\sigma$ by a small positive quantity $\epsilon$. We can therefore use the distributions $\theta_{ \pm}(-\sigma-\epsilon)$ and $\delta_{ \pm}(\sigma+\epsilon)$ in some sensitive computations, and then take the limit $\epsilon \rightarrow 0^{+}$. Notice that the equation $\sigma+\epsilon=0$ describes a two-branch hyperboloid that is located just within the light cone of the reference point $x^{\prime}$. The hyperboloid does not include $x^{\prime}$, and $\theta_{+}(x, \Sigma)$ is one everywhere on its future branch, while $\theta_{-}(x, \Sigma)$ is one everywhere on its past branch. These factors, therefore, become invisible to differential operators. For example, $\theta_{+}^{\prime}(-\sigma-\epsilon)=$ $\theta_{+}(x, \Sigma) \theta^{\prime}(-\sigma-\epsilon)=-\theta_{+}(x, \Sigma) \delta(\sigma+\epsilon)=-\delta_{+}(\sigma+\epsilon)$. This manipulation shows that after the shift from $\sigma$ to $\sigma+\epsilon$, the distributions of Equations (246) and (247) can be straightforwardly differentiated with respect to $\sigma$.

In the next paragraphs we shall establish the distributional identities

$$
\begin{aligned}
\lim _{\epsilon \rightarrow 0^{+}} \epsilon \delta_{ \pm}(\sigma+\epsilon) & =0, \\
\lim _{\epsilon \rightarrow 0^{+}} \epsilon \delta_{ \pm}^{\prime}(\sigma+\epsilon) & =0, \\
\lim _{\epsilon \rightarrow 0^{+}} \epsilon \delta_{ \pm}^{\prime \prime}(\sigma+\epsilon) & =2 \pi \delta_{4}\left(x-x^{\prime}\right)
\end{aligned}
$$

in four-dimensional flat spacetime. These will be used in the next Section 4.1.6 to recover the Green's functions for the scalar wave equation, and they will be generalized to curved spacetime in Section 4.2.

The derivation of Equations $(248,249,250)$ relies on a "master" distributional identity, formulated in three-dimensional flat space:

$$
\lim _{\epsilon \rightarrow 0^{+}} \frac{\epsilon}{R^{5}}=\frac{2 \pi}{3} \delta_{3}(\boldsymbol{x}), \quad R \equiv \sqrt{r^{2}+2 \epsilon},
$$

with $r \equiv|\boldsymbol{x}| \equiv \sqrt{x^{2}+y^{2}+z^{2}}$. This follows from yet another identity, $\nabla^{2} r^{-1}=-4 \pi \delta_{3}(\boldsymbol{x})$, in which we write the left-hand side as $\lim _{\epsilon=0^{+}} \nabla^{2} R^{-1}$; since $R^{-1}$ is nonsingular at $\boldsymbol{x}=0$, it can be straightforwardly differentiated, and the result is $\nabla^{2} R^{-1}=-6 \epsilon / R^{5}$, from which Equation (251) follows.

To prove Equation (248) we must show that $\epsilon \delta_{ \pm}(\sigma+\epsilon)$ vanishes as a distribution in the limit $\epsilon \rightarrow 0^{+}$. For this we must prove that a functional of the form

$$
A_{ \pm}[f]=\lim _{\epsilon \rightarrow 0^{+}} \int \epsilon \delta_{ \pm}(\sigma+\epsilon) f(x) d^{4} x,
$$


where $f(x)=f(t, \boldsymbol{x})$ is a smooth test function, vanishes for all such functions $f$. Our first task will be to find a more convenient expression for $\delta_{ \pm}(\sigma+\epsilon)$. Once more we set $x^{\prime}=0$ (without loss of generality) and we note that $2(\sigma+\epsilon)=-t^{2}+r^{2}+2 \epsilon=-(t-R)(t+R)$, where we have used Equation (251). It follows that

$$
\delta_{ \pm}(\sigma+\epsilon)=\frac{\delta(t \mp R)}{R}
$$

and from this we find

$$
A_{ \pm}[f]=\lim _{\epsilon \rightarrow 0^{+}} \int \epsilon \frac{f( \pm R, \boldsymbol{x})}{R} d^{3} x=\lim _{\epsilon \rightarrow 0^{+}} \int \frac{\epsilon}{R^{5}} R^{4} f( \pm R, \boldsymbol{x}) d^{3} x=\frac{2 \pi}{3} \int \delta_{3}(\boldsymbol{x}) r^{4} f( \pm r, \boldsymbol{x}) d^{3} x=0
$$

which establishes Equation (248).

The validity of Equation (249) is established by a similar computation. Here we must show that a functional of the form

$$
B_{ \pm}[f]=\lim _{\epsilon \rightarrow 0^{+}} \int \epsilon \delta_{ \pm}^{\prime}(\sigma+\epsilon) f(x) d^{4} x
$$

vanishes for all test functions $f$. We have

$$
\begin{aligned}
B_{ \pm}[f] & =\lim _{\epsilon \rightarrow 0^{+}} \epsilon \frac{d}{d \epsilon} \int \delta_{ \pm}(\sigma+\epsilon) f(x) d^{4} x=\lim _{\epsilon \rightarrow 0^{+}} \epsilon \frac{d}{d \epsilon} \int \frac{f( \pm R, \boldsymbol{x})}{R} d^{3} x=\lim _{\epsilon \rightarrow 0^{+}} \epsilon \int\left( \pm \frac{\dot{f}}{R^{2}}-\frac{f}{R^{3}}\right) d^{3} x \\
& =\lim _{\epsilon \rightarrow 0^{+}} \int \frac{\epsilon}{R^{5}}\left( \pm R^{3} \dot{f}-R^{2} f\right) d^{3} x=\frac{2 \pi}{3} \int \delta_{3}(\boldsymbol{x})\left( \pm r^{3} \dot{f}-r^{2} f\right) d^{3} x \\
& =0,
\end{aligned}
$$

and the identity of Equation (249) is proved. In these manipulations we have let an overdot indicate partial differentiation with respect to $t$, and we have used $\partial R / \partial \epsilon=1 / R$.

To establish Equation (250) we consider the functional

$$
C_{ \pm}[f]=\lim _{\epsilon \rightarrow 0^{+}} \int \epsilon \delta_{ \pm}^{\prime \prime}(\sigma+\epsilon) f(x) d^{4} x
$$

and show that it evaluates to $2 \pi f(0, \mathbf{0})$. We have

$$
\begin{aligned}
C_{ \pm}[f] & =\lim _{\epsilon \rightarrow 0^{+}} \epsilon \frac{d^{2}}{d \epsilon^{2}} \int \delta_{ \pm}(\sigma+\epsilon) f(x) d^{4} x=\lim _{\epsilon \rightarrow 0^{+}} \epsilon \frac{d^{2}}{d \epsilon^{2}} \int \frac{f( \pm R, \boldsymbol{x})}{R} d^{3} x \\
& =\lim _{\epsilon \rightarrow 0^{+}} \epsilon \int\left(\frac{\ddot{f}}{R^{3}} \mp 3 \frac{\dot{f}}{R^{4}}+3 \frac{f}{R^{5}}\right) d^{3} x=2 \pi \int \delta_{3}(\boldsymbol{x})\left(\frac{1}{3} r^{2} \ddot{f} \pm r \dot{f}+f\right) d^{3} x \\
& =2 \pi f(0, \mathbf{0}),
\end{aligned}
$$

as required. This proves that Equation (250) holds as a distributional identity in four-dimensional flat spacetime.

\subsubsection{Alternative computation of the Green's functions}

The retarded and advanced Green's functions for the scalar wave equation are now defined as the limit of the functions $G_{ \pm}^{\epsilon}\left(x, x^{\prime}\right)$ as $\epsilon \rightarrow 0^{+}$. For these we make the ansatz

$$
G_{ \pm}^{\epsilon}\left(x, x^{\prime}\right)=\delta_{ \pm}(\sigma+\epsilon)+V(\sigma) \theta_{ \pm}(-\sigma-\epsilon),
$$

and we shall prove that $G_{ \pm}^{\epsilon}\left(x, x^{\prime}\right)$ satisfies Equation (235) in the limit. We recall that the distributions $\theta_{ \pm}$and $\delta_{ \pm}$were defined in the preceding Section 4.1.5, and we assume that $V(\sigma)$ is a smooth

Living Reviews in Relativity

http://www. livingreviews . org/lrr-2004-6 
function of $\sigma\left(x, x^{\prime}\right)=\frac{1}{2} \eta_{\alpha \beta}\left(x-x^{\prime}\right)^{\alpha}\left(x-x^{\prime}\right)^{\beta}$; because this function is smooth, it is not necessary to evaluate $V$ at $\sigma+\epsilon$ in Equation (253). We recall also that $\theta_{+}$and $\delta_{+}$are nonzero when $x$ is in the future of $x^{\prime}$, while $\theta_{-}$and $\delta_{-}$are nonzero when $x$ is in the past of $x^{\prime}$. We will therefore prove that the retarded and advanced Green's functions are of the form

$$
G_{\mathrm{ret}}\left(x, x^{\prime}\right)=\lim _{\epsilon \rightarrow 0^{+}} G_{+}^{\epsilon}\left(x, x^{\prime}\right)=\theta_{+}(x, \Sigma)[\delta(\sigma)+V(\sigma) \theta(-\sigma)]
$$

and

$$
G_{\text {adv }}\left(x, x^{\prime}\right)=\lim _{\epsilon \rightarrow 0^{+}} G_{-}^{\epsilon}\left(x, x^{\prime}\right)=\theta_{-}(x, \Sigma)[\delta(\sigma)+V(\sigma) \theta(-\sigma)],
$$

where $\Sigma$ is a spacelike hypersurface that contains $x^{\prime}$. We will also determine the form of the function $V(\sigma)$.

The functions that appear in Equation (253) can be straightforwardly differentiated. The manipulations are similar to what was done in Section 4.1.4, and dropping all labels, we obtain $\left(\square-k^{2}\right) G=2 \sigma G^{\prime \prime}+4 G^{\prime}-k^{2} G$, with a prime indicating differentiation with respect to $\sigma$. From Equation (253) we obtain $G^{\prime}=\delta^{\prime}-V \delta+V^{\prime} \theta$ and $G^{\prime \prime}=\delta^{\prime \prime}-V \delta^{\prime}-2 V^{\prime} \delta+V^{\prime \prime} \theta$. The identities of Equation (241) can be expressed as $(\sigma+\epsilon) \delta^{\prime}(\sigma+\epsilon)=-\delta(\sigma+\epsilon)$ and $(\sigma+\epsilon) \delta^{\prime \prime}(\sigma+\epsilon)=-2 \delta^{\prime}(\sigma+\epsilon)$, and combining this with our previous results gives

$$
\begin{aligned}
\left(\square-k^{2}\right) G_{ \pm}^{\epsilon}\left(x, x^{\prime}\right)= & \left(-2 V-k^{2}\right) \delta_{ \pm}(\sigma+\epsilon)+\left(2 \sigma V^{\prime \prime}+4 V^{\prime}-k^{2} V\right) \theta_{ \pm}(-\sigma-\epsilon) \\
& -2 \epsilon \delta_{ \pm}^{\prime \prime}(\sigma+\epsilon)+2 V \epsilon \delta_{ \pm}^{\prime}(\sigma+\epsilon)+4 V^{\prime} \epsilon \delta_{ \pm}(\sigma+\epsilon) .
\end{aligned}
$$

According to Equation $(248,249,250)$, the last two terms on the right-hand side disappear in the limit $\epsilon \rightarrow 0^{+}$, and the third term becomes $-4 \pi \delta_{4}\left(x-x^{\prime}\right)$. Provided that the first two terms vanish also, we recover $\left(\square-k^{2}\right) G\left(x, x^{\prime}\right)=-4 \pi \delta_{4}\left(x-x^{\prime}\right)$ in the limit, as required. Thus, the limit of $G_{ \pm}^{\epsilon}\left(x, x^{\prime}\right)$ as $\epsilon \rightarrow 0^{+}$will indeed satisfy Green's equation provided that $V(\sigma)$ is a solution to

$$
2 \sigma V^{\prime \prime}+4 V^{\prime}-k^{2} V=0, \quad V(0)=-\frac{1}{2} k^{2}
$$

these are the same statements as in Equation (244). The solution to these equations was produced in Equation (245),

$$
V(\sigma)=-\frac{k}{\sqrt{-2 \sigma}} J_{1}(k \sqrt{-2 \sigma})
$$

and this completely determines the Green's functions of Equations (254) and (255).

\subsection{Distributions in curved spacetime}

The distributions introduced in Section 4.1.5 can also be defined in a four-dimensional spacetime with metric $g_{\alpha \beta}$. Here we produce the relevant generalizations of the results derived in that section.

\subsubsection{Invariant Dirac distribution}

We first introduce $\delta_{4}\left(x, x^{\prime}\right)$, an invariant Dirac functional in a four-dimensional curved spacetime. This is defined by the relations

$$
\int_{V} f(x) \delta_{4}\left(x, x^{\prime}\right) \sqrt{-g} d^{4} x=f\left(x^{\prime}\right), \quad \int_{V^{\prime}} f\left(x^{\prime}\right) \delta_{4}\left(x, x^{\prime}\right) \sqrt{-g^{\prime}} d^{4} x^{\prime}=f(x),
$$

where $f(x)$ is a smooth test function, $V$ any four-dimensional region that contains $x^{\prime}$, and $V^{\prime}$ any four-dimensional region that contains $x$. These relations imply that $\delta_{4}\left(x, x^{\prime}\right)$ is symmetric in its arguments, and it is easy to see that

$$
\delta_{4}\left(x, x^{\prime}\right)=\frac{\delta_{4}\left(x-x^{\prime}\right)}{\sqrt{-g}}=\frac{\delta_{4}\left(x-x^{\prime}\right)}{\sqrt{-g^{\prime}}}=\left(g g^{\prime}\right)^{-1 / 4} \delta_{4}\left(x-x^{\prime}\right),
$$


where $\delta_{4}\left(x-x^{\prime}\right)=\delta\left(x^{0}-x^{\prime 0}\right) \delta\left(x^{1}-x^{\prime 1}\right) \delta\left(x^{2}-x^{\prime 2}\right) \delta\left(x^{3}-x^{\prime 3}\right)$ is the ordinary (coordinate) four-dimensional Dirac functional. The relations of Equation (259) are all equivalent because $f(x) \delta_{4}\left(x, x^{\prime}\right)=f\left(x^{\prime}\right) \delta_{4}\left(x, x^{\prime}\right)$ is a distributional identity; the last form is manifestly symmetric in $x$ and $x^{\prime}$.

The invariant Dirac distribution satisfies the identities

$$
\begin{gathered}
\Omega_{\ldots}\left(x, x^{\prime}\right) \delta_{4}\left(x, x^{\prime}\right)=\left[\Omega_{\ldots}\right] \delta_{4}\left(x, x^{\prime}\right), \\
\left(g_{\alpha^{\prime}}^{\alpha}\left(x, x^{\prime}\right) \delta_{4}\left(x, x^{\prime}\right)\right)_{; \alpha}=-\partial_{\alpha^{\prime}} \delta_{4}\left(x, x^{\prime}\right), \quad\left(g_{\alpha}^{\alpha^{\prime}}\left(x^{\prime}, x\right) \delta_{4}\left(x, x^{\prime}\right)\right)_{; \alpha^{\prime}}=-\partial_{\alpha} \delta_{4}\left(x, x^{\prime}\right),
\end{gathered}
$$

where $\Omega_{\ldots}\left(x, x^{\prime}\right)$ is any bitensor and $g_{\alpha^{\prime}}^{\alpha}\left(x, x^{\prime}\right), g_{\alpha}^{\alpha^{\prime}}\left(x, x^{\prime}\right)$ are parallel propagators. The first identity follows immediately from the definition of the $\delta$-function. The second and third identities are established by showing that integration against a test function $f(x)$ gives the same result from both sides. For example, the first of the Equations (258) implies

$$
\int_{V} f(x) \partial_{\alpha^{\prime}} \delta_{4}\left(x, x^{\prime}\right) \sqrt{-g} d^{4} x=\partial_{\alpha^{\prime}} f\left(x^{\prime}\right)
$$

and on the other hand,

$$
-\int_{V} f(x)\left(g_{\alpha^{\prime}}^{\alpha} \delta_{4}\left(x, x^{\prime}\right)\right)_{; \alpha} \sqrt{-g} d^{4} x=-\oint_{\partial V} f(x) g_{\alpha^{\prime}}^{\alpha} \delta_{4}\left(x, x^{\prime}\right) d \Sigma_{\alpha}+\left[f_{, \alpha} g_{\alpha^{\prime}}^{\alpha}\right]=\partial_{\alpha^{\prime}} f\left(x^{\prime}\right),
$$

which establishes the second identity of Equation (260). Notice that in these manipulations, the integrations involve scalar functions of the coordinates $x$; the fact that these functions are also vectors with respect to $x^{\prime}$ does not invalidate the procedure. The third identity of Equation (260) is proved in a similar way.

\subsubsection{Light-cone distributions}

For the remainder of Section 4.2 we assume that $x \in \mathcal{N}\left(x^{\prime}\right)$, so that a unique geodesic $\beta$ links these two points. We then let $\sigma\left(x, x^{\prime}\right)$ be the curved spacetime world function, and we define light-cone step functions by

$$
\theta_{ \pm}(-\sigma)=\theta_{ \pm}(x, \Sigma) \theta(-\sigma), \quad x^{\prime} \in \Sigma,
$$

where $\theta_{+}(x, \Sigma)$ is one if $x$ is in the future of the spacelike hypersurface $\Sigma$ and zero otherwise, and $\theta_{-}(x, \Sigma)=1-\theta_{+}(x, \Sigma)$. These are immediate generalizations to curved spacetime of the objects defined in flat spacetime by Equation (246). We have that $\theta_{+}(-\sigma)$ is one if $x$ is an element of $I^{+}\left(x^{\prime}\right)$, the chronological future of $x^{\prime}$, and zero otherwise, and $\theta_{-}(-\sigma)$ is one if $x$ is an element of $I^{-}\left(x^{\prime}\right)$, the chronological past of $x^{\prime}$, and zero otherwise. We also have $\theta_{+}(-\sigma)+\theta_{-}(-\sigma)=\theta(-\sigma)$.

We define the curved-spacetime version of the light-cone Dirac functionals by

$$
\delta_{ \pm}(\sigma)=\theta_{ \pm}(x, \Sigma) \delta(\sigma), \quad x^{\prime} \in \Sigma,
$$

an immediate generalization of Equation (247). We have that $\delta_{+}(\sigma)$, when viewed as a function of $x$, is supported on the future light cone of $x^{\prime}$, while $\delta_{-}(\sigma)$ is supported on its past light cone. We also have $\delta_{+}(\sigma)+\delta_{-}(\sigma)=\delta(\sigma)$, and we recall that $\sigma$ is negative if $x$ and $x^{\prime}$ are timelike related, and positive if they are spacelike related.

For the same reasons as those mentioned in Section 4.1.5, it is sometimes convenient to shift the argument of the step and $\delta$-functions from $\sigma$ to $\sigma+\epsilon$, where $\epsilon$ is a small positive quantity. With this shift, the light-cone distributions can be straightforwardly differentiated with respect to $\sigma$. For example, $\delta_{ \pm}(\sigma+\epsilon)=-\theta_{ \pm}^{\prime}(-\sigma-\epsilon)$, with a prime indicating differentiation with respect to $\sigma$.

Living Reviews in Relativity

http: //www . livingreviews . org/lrr-2004-6 
We now prove that the identities of Equation $(248,249,250)$ generalize to

$$
\begin{aligned}
\lim _{\epsilon \rightarrow 0^{+}} \epsilon \delta_{ \pm}(\sigma+\epsilon) & =0, \\
\lim _{\epsilon \rightarrow 0^{+}} \epsilon \delta_{ \pm}^{\prime}(\sigma+\epsilon) & =0, \\
\lim _{\epsilon \rightarrow 0^{+}} \epsilon \delta_{ \pm}^{\prime \prime}(\sigma+\epsilon) & =2 \pi \delta_{4}\left(x, x^{\prime}\right)
\end{aligned}
$$

in a four-dimensional curved spacetime; the only differences lie with the definition of the world function and the fact that it is the invariant Dirac functional that appears in Equation (265). To establish these identities in curved spacetime we use the fact that they hold in flat spacetime - as was shown in Section 4.1.5 - and that they are scalar relations that must be valid in any coordinate system if they are found to hold in one. Let us then examine Equations $(263,264)$ in the Riemann normal coordinates of Section 3.1; these are denoted $\hat{x}^{\alpha}$ and are based at $x^{\prime}$. We have that $\sigma\left(x, x^{\prime}\right)=\frac{1}{2} \eta_{\alpha \beta} \hat{x}^{\alpha} \hat{x}^{\beta}$ and $\delta_{4}\left(x, x^{\prime}\right)=\Delta\left(x, x^{\prime}\right) \delta_{4}\left(x-x^{\prime}\right)=\delta_{4}\left(x-x^{\prime}\right)$, where $\Delta\left(x, x^{\prime}\right)$ is the van Vleck determinant, whose coincidence limit is unity. In Riemann normal coordinates, therefore, Equations $(263,264,265)$ take exactly the same form as Equations $(248,264,250)$. Because the identities are true in flat spacetime, they must be true also in curved spacetime (in Riemann normal coordinates based at $x^{\prime}$ ); and because these are scalar relations, they must be valid in any coordinate system.

\subsection{Scalar Green's functions in curved spacetime}

\subsubsection{Green's equation for a massless scalar field in curved spacetime}

We consider a massless scalar field $\Phi(x)$ in a curved spacetime with metric $g_{\alpha \beta}$. The field satisfies the wave equation

$$
(\square-\xi R) \Phi(x)=-4 \pi \mu(x),
$$

where $\square=g^{\alpha \beta} \nabla_{\alpha} \nabla_{\beta}$ is the wave operator, $R$ the Ricci scalar, $\xi$ an arbitrary coupling constant, and $\mu(x)$ is a prescribed source. We seek a Green's function $G\left(x, x^{\prime}\right)$ such that a solution to Equation (266) can be expressed as

$$
\Phi(x)=\int G\left(x, x^{\prime}\right) \mu\left(x^{\prime}\right) \sqrt{-g^{\prime}} d^{4} x^{\prime},
$$

where the integration is over the entire spacetime. The wave equation for the Green's function is

$$
(\square-\xi R) G\left(x, x^{\prime}\right)=-4 \pi \delta_{4}\left(x, x^{\prime}\right),
$$

where $\delta_{4}\left(x, x^{\prime}\right)$ is the invariant Dirac functional introduced in Section 4.2.1. It is easy to verify that the field defined by Equation (267) is truly a solution to Equation (266).

We let $G_{+}\left(x, x^{\prime}\right)$ be the retarded solution to Equation (268), and $G_{-}\left(x, x^{\prime}\right)$ be the advanced solution; when viewed as functions of $x, G_{+}\left(x, x^{\prime}\right)$ is nonzero in the causal future of $x^{\prime}$, while $G_{-}\left(x, x^{\prime}\right)$ is nonzero in its causal past. We assume that the retarded and advanced Green's functions exist as distributions and can be defined globally in the entire spacetime.

\subsubsection{Hadamard construction of the Green's functions}

Assuming throughout this section that $x$ is restricted to the normal convex neighbourhood of $x^{\prime}$, we make the ansatz

$$
G_{ \pm}\left(x, x^{\prime}\right)=U\left(x, x^{\prime}\right) \delta_{ \pm}(\sigma)+V\left(x, x^{\prime}\right) \theta_{ \pm}(-\sigma),
$$

where $U\left(x, x^{\prime}\right)$ and $V\left(x, x^{\prime}\right)$ are smooth biscalars; the fact that the spacetime is no longer homogeneous means that these functions cannot depend on $\sigma$ alone. 
Before we substitute the Green's functions of Equation (269) into the differential equation of Equation (268), we proceed as in Section 4.1 .6 and shift $\sigma$ by the small positive quantity $\epsilon$. We shall therefore consider the distributions

$$
G_{ \pm}^{\epsilon}\left(x, x^{\prime}\right)=U\left(x, x^{\prime}\right) \delta_{ \pm}(\sigma+\epsilon)+V\left(x, x^{\prime}\right) \theta_{ \pm}(-\theta-\epsilon),
$$

and later recover the Green's functions by taking the limit $\epsilon \rightarrow 0^{+}$. Differentiation of these objects is straightforward, and in the following manipulations we will repeatedly use the relation $\sigma^{\alpha} \sigma_{\alpha}=2 \sigma$ satisfied by the world function. We will also use the distributional identities $\sigma \delta_{ \pm}(\sigma+$ $\epsilon)=-\epsilon \delta_{ \pm}(\sigma+\epsilon), \sigma \delta_{ \pm}^{\prime}(\sigma+\epsilon)=-\delta_{ \pm}(\sigma+\epsilon)-\epsilon \delta_{ \pm}^{\prime}(\sigma+\epsilon)$, and $\sigma \delta_{ \pm}^{\prime \prime}(\sigma+\epsilon)=-2 \delta^{\prime}(\sigma+\epsilon)-\epsilon \delta^{\prime \prime}(\sigma+\epsilon)$ After a routine calculation we obtain

$$
\begin{aligned}
(\square-\xi R) G_{ \pm}^{\epsilon}= & -2 \epsilon \delta_{ \pm}^{\prime \prime}(\sigma+\epsilon) U+2 \epsilon \delta_{ \pm}^{\prime}(\sigma+\epsilon) V+\delta_{ \pm}^{\prime}(\sigma+\epsilon)\left\{2 U_{, \alpha} \sigma^{\alpha}+\left(\sigma_{\alpha}^{\alpha}-4\right) U\right\} \\
& +\delta_{ \pm}(\sigma+\epsilon)\left\{-2 V_{, \alpha} \sigma^{\alpha}+\left(2-\sigma_{\alpha}^{\alpha}\right) V+(\square-\xi R) U\right\}+\theta_{ \pm}(-\sigma-\epsilon)\{(\square-\xi R) V\}
\end{aligned}
$$

which becomes

$$
\begin{aligned}
(\square-\xi R) G_{ \pm}= & -4 \pi \delta_{4}\left(x, x^{\prime}\right) U+\delta_{ \pm}^{\prime}(\sigma)\left\{2 U_{, \alpha} \sigma^{\alpha}+\left(\sigma_{\alpha}^{\alpha}-4\right) U\right\} \\
& +\delta_{ \pm}(\sigma)\left\{-2 V_{, \alpha} \sigma^{\alpha}+\left(2-\sigma_{\alpha}^{\alpha}\right) V+(\square-\xi R) U\right\}+\theta_{ \pm}(-\sigma)\{(\square-\xi R) V\}
\end{aligned}
$$

in the limit $\epsilon \rightarrow 0^{+}$, after using the identities of Equations (263, 264, 265).

According to Equation (268), the right-hand side of Equation (270) should be equal to $-4 \pi \delta_{4}\left(x, x^{\prime}\right)$. This immediately gives us the coincidence condition

$$
[U]=1
$$

for the biscalar $U\left(x, x^{\prime}\right)$. To eliminate the $\delta_{ \pm}^{\prime}$ term we make its coefficient vanish:

$$
2 U_{, \alpha} \sigma^{\alpha}+\left(\sigma_{\alpha}^{\alpha}-4\right) U=0
$$

As we shall now prove, these two equations determine $U\left(x, x^{\prime}\right)$ uniquely.

Recall from Section 2.1.3 that $\sigma^{\alpha}$ is a vector at $x$ that is tangent to the unique geodesic $\beta$ that connects $x$ to $x^{\prime}$. This geodesic is affinely parameterized by $\lambda$ and a displacement along $\beta$ is described by $d x^{\alpha}=\left(\sigma^{\alpha} / \lambda\right) d \lambda$. The first term of Equation (272) therefore represents the rate of change of $U\left(x, x^{\prime}\right)$ along $\beta$, and this can be expressed as $2 \lambda d U / d \lambda$. For the second term we recall from Section 2.5.1 the differential equation $\Delta^{-1}\left(\Delta \sigma^{\alpha}\right)_{; \alpha}=4$ satisfied by $\Delta\left(x, x^{\prime}\right)$, the van Vleck determinant. This gives us $\sigma_{\alpha}^{\alpha}-4=\Delta^{-1} \Delta_{, \alpha} \sigma^{\alpha}=\Delta^{-1} \lambda d \Delta / d \lambda$, and Equation (272) becomes

$$
\lambda \frac{d}{d \lambda}(2 \ln U-\ln \Delta)=0 .
$$

It follows that $U^{2} / \Delta$ is constant on $\beta$, and it must therefore be equal to its value at the starting point $x^{\prime}: U^{2} / \Delta=\left[U^{2} / \Delta\right]=1$, by virtue of Equation (271) and the property $[\Delta]=1$ of the van Vleck determinant. Since this statement must be true for all geodesics $\beta$ that emanate from $x^{\prime}$, we have found that the unique solution to Equations (271) and (272) is

$$
U\left(x, x^{\prime}\right)=\Delta^{1 / 2}\left(x, x^{\prime}\right)
$$

We must still consider the remaining terms in Equation (270). The $\delta_{ \pm}$term can be eliminated by demanding that its coefficient vanish when $\sigma=0$. This, however, does not constrain its value away from the light cone, and we thus obtain information about $\left.V\right|_{\sigma=0}$ only. Denoting this by $\check{V}\left(x, x^{\prime}\right)$ - the restriction of $V\left(x, x^{\prime}\right)$ on the light cone $\sigma\left(x, x^{\prime}\right)=0$ - we have

$$
\check{V}_{, \alpha} \sigma^{\alpha}+\frac{1}{2}\left(\sigma_{\alpha}^{\alpha}-2\right) \check{V}=\left.\frac{1}{2}(\square-\xi R) U\right|_{\sigma=0},
$$

Living Reviews in Relativity

http: //www . livingreviews . org/lrr-2004-6 
where we indicate that the right-hand side also must be restricted to the light cone. The first term of Equation (274) can be expressed as $\lambda d \check{V} / d \lambda$, and this equation can be integrated along any null geodesic that generates the null cone $\sigma\left(x, x^{\prime}\right)=0$. For these integrations to be well posed, however, we must provide initial values at $x=x^{\prime}$. As we shall now see, these can be inferred from Equation (274) and the fact that $V\left(x, x^{\prime}\right)$ must be smooth at coincidence.

Equations (97) and (273) imply that near coincidence, $U\left(x, x^{\prime}\right)$ admits the expansion

$$
U=1+\frac{1}{12} R_{\alpha^{\prime} \beta^{\prime}} \sigma^{\alpha^{\prime}} \sigma^{\beta^{\prime}}+\mathcal{O}\left(\lambda^{3}\right),
$$

where $R_{\alpha^{\prime} \beta^{\prime}}$ is the Ricci tensor at $x^{\prime}$ and $\lambda$ is the affine-parameter distance to $x$ (which can be either on or off the light cone). Differentiation of this relation gives

$$
U_{, \alpha}=-\frac{1}{6} g_{\alpha}^{\alpha^{\prime}} R_{\alpha^{\prime} \beta^{\prime}} \sigma^{\beta^{\prime}}+\mathcal{O}\left(\lambda^{2}\right), \quad U_{, \alpha^{\prime}}=\frac{1}{6} R_{\alpha^{\prime} \beta^{\prime}} \sigma^{\beta^{\prime}}+\mathcal{O}\left(\lambda^{2}\right),
$$

and eventually,

$$
[\square U]=\frac{1}{6} R\left(x^{\prime}\right) .
$$

Using also $\left[\sigma_{\alpha}^{\alpha}\right]=4$, we find that the coincidence limit of Equation (274) gives

$$
[V]=\frac{1}{12}(1-6 \xi) R\left(x^{\prime}\right),
$$

and this provides the initial values required for the integration of Equation (274) on the null cone.

Equations (274) and (278) give us a means to construct $\check{V}\left(x, x^{\prime}\right)$, the restriction of $V\left(x, x^{\prime}\right)$ on the null cone $\sigma\left(x, x^{\prime}\right)=0$. These values can then be used as characteristic data for the wave equation

$$
(\square-\xi R) V\left(x, x^{\prime}\right)=0,
$$

which is obtained by elimination of the $\theta_{ \pm}$term in Equation (270). While this certainly does not constitute a practical method to compute the biscalar $V\left(x, x^{\prime}\right)$, these considerations show that $V\left(x, x^{\prime}\right)$ exists and is unique.

To summarize: We have shown that with $U\left(x, x^{\prime}\right)$ given by Equation $(273)$ and $V\left(x, x^{\prime}\right)$ determined uniquely by the wave equation of Equation (279) and the characteristic data constructed with Equations (274) and (278), the retarded and advanced Green's functions of Equation (269) do indeed satisfy Equation (268). It should be emphasized that the construction provided in this section is restricted to $\mathcal{N}\left(x^{\prime}\right)$, the normal convex neighbourhood of the reference point $x^{\prime}$.

\subsubsection{Reciprocity}

We shall now establish the following reciprocity relation between the (globally defined) retarded and advanced Green's functions:

$$
G_{-}\left(x^{\prime}, x\right)=G_{+}\left(x, x^{\prime}\right) .
$$

Before we get to the proof we observe that by virtue of Equation (280), the biscalar $V\left(x, x^{\prime}\right)$ must be symmetric in its arguments,

$$
V\left(x^{\prime}, x\right)=V\left(x, x^{\prime}\right) .
$$

To go from Equation (280) to Equation (281) we need simply note that if $x \in \mathcal{N}\left(x^{\prime}\right)$ and belongs to $I^{+}\left(x^{\prime}\right)$, then $G_{+}\left(x, x^{\prime}\right)=V\left(x, x^{\prime}\right)$ and $G_{-}\left(x^{\prime}, x\right)=V\left(x^{\prime}, x\right)$.

To prove the reciprocity relation we invoke the identities

$$
G_{+}\left(x, x^{\prime}\right)(\square-\xi R) G_{-}\left(x, x^{\prime \prime}\right)=-4 \pi G_{+}\left(x, x^{\prime}\right) \delta_{4}\left(x, x^{\prime \prime}\right)
$$


and

$$
G_{-}\left(x, x^{\prime \prime}\right)(\square-\xi R) G_{+}\left(x, x^{\prime}\right)=-4 \pi G_{-}\left(x, x^{\prime \prime}\right) \delta_{4}\left(x, x^{\prime}\right),
$$

and take their difference. On the left-hand side we have

$$
\begin{aligned}
& G_{+}\left(x, x^{\prime}\right) \square G_{-}\left(x, x^{\prime \prime}\right)-G_{-}\left(x, x^{\prime \prime}\right) \square G_{+}\left(x, x^{\prime}\right)= \\
& \quad \nabla_{\alpha}\left(G_{+}\left(x, x^{\prime}\right) \nabla^{\alpha} G_{-}\left(x, x^{\prime \prime}\right)-G_{-}\left(x, x^{\prime \prime}\right) \nabla^{\alpha} G_{+}\left(x, x^{\prime}\right)\right),
\end{aligned}
$$

while the right-hand side gives

$$
-4 \pi\left(G_{+}\left(x, x^{\prime}\right) \delta_{4}\left(x, x^{\prime \prime}\right)-G_{-}\left(x, x^{\prime \prime}\right) \delta_{4}\left(x, x^{\prime}\right)\right) .
$$

Integrating both sides over a large four-dimensional region $V$ that contains both $x^{\prime}$ and $x^{\prime \prime}$, we obtain

$$
\oint_{\partial V}\left(G_{+}\left(x, x^{\prime}\right) \nabla^{\alpha} G_{-}\left(x, x^{\prime \prime}\right)-G_{-}\left(x, x^{\prime \prime}\right) \nabla^{\alpha} G_{+}\left(x, x^{\prime}\right)\right) d \Sigma_{\alpha}=-4 \pi\left(G_{+}\left(x^{\prime \prime}, x^{\prime}\right)-G_{-}\left(x^{\prime}, x^{\prime \prime}\right)\right),
$$

where $\partial V$ is the boundary of $V$. Assuming that the Green's functions fall off sufficiently rapidly at infinity (in the limit $\partial V \rightarrow \infty$; this statement imposes some restriction on the spacetime's asymptotic structure), we have that the left-hand side of the equation evaluates to zero in the limit. This gives us the statement $G_{+}\left(x^{\prime \prime}, x^{\prime}\right)=G_{-}\left(x^{\prime}, x^{\prime \prime}\right)$, which is just Equation (280) with $x^{\prime \prime}$ replacing $x$.

\subsubsection{Kirchhoff representation}

Suppose that the values for a scalar field $\Phi\left(x^{\prime}\right)$ and its normal derivative $n^{\alpha^{\prime}} \nabla_{\alpha^{\prime}} \Phi\left(x^{\prime}\right)$ are known on a spacelike hypersurface $\Sigma$. Suppose also that the scalar field satisfies the homogeneous wave equation

$$
(\square-\xi R) \Phi(x)=0 .
$$

Then the value of the field at a point $x$ in the future of $\Sigma$ is given by Kirchhoff's formula,

$$
\Phi(x)=-\frac{1}{4 \pi} \int_{\Sigma}\left(G_{+}\left(x, x^{\prime}\right) \nabla^{\alpha^{\prime}} \Phi\left(x^{\prime}\right)-\Phi\left(x^{\prime}\right) \nabla^{\alpha^{\prime}} G_{+}\left(x, x^{\prime}\right)\right) d \Sigma_{\alpha^{\prime}}
$$

where $d \Sigma_{\alpha^{\prime}}$ is the surface element on $\Sigma$. If $n_{\alpha^{\prime}}$ is the future-directed unit normal, then $d \Sigma_{\alpha^{\prime}}=$ $-n_{\alpha^{\prime}} d V$, with $d V$ denoting the invariant volume element on $\Sigma$; notice that $d \Sigma_{\alpha^{\prime}}$ is past directed.

To establish this result we start with the equations

$$
G_{-}\left(x^{\prime}, x\right)\left(\square^{\prime}-\xi R^{\prime}\right) \Phi\left(x^{\prime}\right)=0, \quad \Phi\left(x^{\prime}\right)\left(\square^{\prime}-\xi R^{\prime}\right) G_{-}\left(x^{\prime}, x\right)=-4 \pi \delta_{4}\left(x^{\prime}, x\right) \Phi\left(x^{\prime}\right),
$$

in which $x$ and $x^{\prime}$ refer to arbitrary points in spacetime. Taking their difference gives

$$
\nabla_{\alpha^{\prime}}\left(G_{-}\left(x^{\prime}, x\right) \nabla^{\alpha^{\prime}} \Phi\left(x^{\prime}\right)-\Phi\left(x^{\prime}\right) \nabla^{\alpha^{\prime}} G_{-}\left(x^{\prime}, x\right)\right)=4 \pi \delta_{4}\left(x^{\prime}, x\right) \Phi\left(x^{\prime}\right),
$$

and this we integrate over a four-dimensional region $V$ that is bounded in the past by the hypersurface $\Sigma$. We suppose that $V$ contains $x$ and we obtain

$$
\oint_{\partial V}\left(G_{-}\left(x^{\prime}, x\right) \nabla^{\alpha^{\prime}} \Phi\left(x^{\prime}\right)-\Phi\left(x^{\prime}\right) \nabla^{\alpha^{\prime}} G_{-}\left(x^{\prime}, x\right)\right) d \Sigma_{\alpha^{\prime}}=4 \pi \Phi(x),
$$

where $d \Sigma_{\alpha^{\prime}}$ is the outward-directed surface element on the boundary $\partial V$. Assuming that the Green's function falls off sufficiently rapidly into the future, we have that the only contribution to the hypersurface integral is the one that comes from $\Sigma$. Since the surface element on $\Sigma$ points in

Living Reviews in Relativity

http: //www . livingreviews.org/lrr-2004-6 
the direction opposite to the outward-directed surface element on $\partial V$, we must change the sign of the left-hand side to be consistent with the convention adopted previously. With this change we have

$$
\Phi(x)=-\frac{1}{4 \pi} \oint_{\partial V}\left(G_{-}\left(x^{\prime}, x\right) \nabla^{\alpha^{\prime}} \Phi\left(x^{\prime}\right)-\Phi\left(x^{\prime}\right) \nabla^{\alpha^{\prime}} G_{-}\left(x^{\prime}, x\right)\right) d \Sigma_{\alpha^{\prime}},
$$

which is the same as Equation (283) if we take into account the reciprocity relation of Equation (280).

\subsubsection{Singular and radiative Green's functions}

In Section 5 of this review we will compute the retarded field of a moving scalar charge, and we will analyze its singularity structure near the world line; this will be part of our effort to understand the effect of the field on the particle's motion. The retarded solution to the scalar wave equation is the physically relevant solution because it properly incorporates outgoing-wave boundary conditions at infinity - the advanced solution would come instead with incoming-wave boundary conditions. The retarded field is singular on the world line because a point particle produces a Coulomb field that diverges at the particle's position. In view of this singular behaviour, it is a subtle matter to describe the field's action on the particle, and to formulate meaningful equations of motion.

When facing this problem in flat spacetime (recall the discussion of Section 1.3), it is convenient to decompose the retarded Green's function $G_{+}\left(x, x^{\prime}\right)$ into a singular Green's function $G_{\mathrm{S}}\left(x, x^{\prime}\right) \equiv$ $\frac{1}{2}\left[G_{+}\left(x, x^{\prime}\right)+G_{-}\left(x, x^{\prime}\right)\right]$ and a radiative Green's function $G_{\mathrm{R}}\left(x, x^{\prime}\right) \equiv \frac{1}{2}\left[G_{+}\left(x, x^{\prime}\right)-G_{-}\left(x, x^{\prime}\right)\right]$. The singular Green's function takes its name from the fact that it produces a field with the same singularity structure as the retarded solution: The diverging field near the particle is insensitive to the boundary conditions imposed at infinity. We note also that $G_{\mathrm{S}}\left(x, x^{\prime}\right)$ satisfies the same wave equation as the retarded Green's function (with a Dirac functional as a source), and that by virtue of the reciprocity relations, it is symmetric in its arguments. The radiative Green's function, on the other hand, takes its name from the fact that it satisfies the homogeneous wave equation, without the Dirac functional on the right-hand side; it produces a field that is smooth on the world line of the moving scalar charge.

Because the singular Green's function is symmetric in its argument, it does not distinguish between past and future, and it produces a field that contains equal amounts of outgoing and incoming radiation - the singular solution describes standing waves at infinity. Removing $G_{\mathrm{S}}\left(x, x^{\prime}\right)$ from the retarded Green's function will therefore have the effect of removing the singular behaviour of the field without affecting the motion of the particle. The motion is not affected because it is intimately tied to the boundary conditions: If the waves are outgoing, the particle loses energy to the radiation and its motion is affected; if the waves are incoming, the particle gains energy from the radiation and its motion is affected differently. With equal amounts of outgoing and incoming radiation, the particle neither loses nor gains energy and its interaction with the scalar field cannot affect its motion. Thus, subtracting $G_{\mathrm{S}}\left(x, x^{\prime}\right)$ from the retarded Green's function eliminates the singular part of the field without affecting the motion of the scalar charge. The subtraction leaves behind the radiative Green's function, which produces a field that is smooth on the world line; it is this field that will govern the motion of the particle. The action of this field is well defined, and it properly encodes the outgoing-wave boundary conditions: The particle will lose energy to the radiation.

In this section we attempt a decomposition of the curved-spacetime retarded Green's function into singular and radiative Green's functions. The flat-spacetime relations will have to be amended, however, because of the fact that in a curved spacetime, the advanced Green's function is generally nonzero when $x^{\prime}$ is in the chronological future of $x$. This implies that the value of the advanced field at $x$ depends on events $x^{\prime}$ that will unfold in the future; this dependence would be inherited 
by the radiative field (which acts on the particle and determines its motion) if the naive definition $G_{\mathrm{R}}\left(x, x^{\prime}\right) \equiv \frac{1}{2}\left[G_{+}\left(x, x^{\prime}\right)-G_{-}\left(x, x^{\prime}\right)\right]$ were to be adopted.

We shall not adopt this definition. Instead, we shall follow Detweiler and Whiting [23] and introduce a singular Green's function with the properties

Sc.S1: $G_{\mathrm{S}}\left(x, x^{\prime}\right)$ satisfies the inhomogeneous scalar wave equation,

$$
(\square-\xi R) G_{\mathrm{S}}\left(x, x^{\prime}\right)=-4 \pi \delta_{4}\left(x, x^{\prime}\right) ;
$$

Sc.S2: $G_{\mathrm{S}}\left(x, x^{\prime}\right)$ is symmetric in its arguments,

$$
G_{\mathrm{S}}\left(x^{\prime}, x\right)=G_{\mathrm{S}}\left(x, x^{\prime}\right)
$$

Sc.S3: $G_{\mathrm{S}}\left(x, x^{\prime}\right)$ vanishes if $x$ is in the chronological past or future of $x^{\prime}$,

$$
G_{\mathrm{S}}\left(x, x^{\prime}\right)=0 \quad \text { when } x \in I^{ \pm}\left(x^{\prime}\right) .
$$

Properties Sc.S1 and Sc.S2 ensure that the singular Green's function will properly reproduce the singular behaviour of the retarded solution without distinguishing between past and future; and as we shall see, Property Sc.S3 ensures that the support of the radiative Green's function will not include the chronological future of $x$.

The radiative Green's function is then defined by

$$
G_{\mathrm{R}}\left(x, x^{\prime}\right)=G_{+}\left(x, x^{\prime}\right)-G_{\mathrm{S}}\left(x, x^{\prime}\right),
$$

where $G_{+}\left(x, x^{\prime}\right)$ is the retarded Green's function. This comes with the properties

Sc.R1: $G_{\mathrm{R}}\left(x, x^{\prime}\right)$ satisfies the homogeneous wave equation,

$$
(\square-\xi R) G_{\mathrm{R}}\left(x, x^{\prime}\right)=0 ;
$$

Sc.R2: $G_{\mathrm{R}}\left(x, x^{\prime}\right)$ agrees with the retarded Green's function if $x$ is in the chronological future of $x^{\prime}$

$$
G_{\mathrm{R}}\left(x, x^{\prime}\right)=G_{+}\left(x, x^{\prime}\right) \quad \text { when } x \in I^{+}\left(x^{\prime}\right) ;
$$

Sc.R3: $G_{\mathrm{R}}\left(x, x^{\prime}\right)$ vanishes if $x$ is in the chronological past of $x^{\prime}$,

$$
G_{\mathrm{R}}\left(x, x^{\prime}\right)=0 \quad \text { when } x \in I^{-}\left(x^{\prime}\right) .
$$

Property Sc.R1 follows directly from Equation (287) and Property Sc.S1 of the singular Green's function. Properties Sc.R2 and Sc.R3 follow from Property Sc.S3 and the fact that the retarded Green's function vanishes if $x$ is in past of $x^{\prime}$. The properties of the radiative Green's function ensure that the corresponding radiative field will be smooth at the world line, and will depend only on the past history of the scalar charge.

We must still show that such singular and radiative Green's functions can be constructed. This relies on the existence of a two-point function $H\left(x, x^{\prime}\right)$ that would possess the properties

Sc.H1: $H\left(x, x^{\prime}\right)$ satisfies the homogeneous wave equation,

$$
(\square-\xi R) H\left(x, x^{\prime}\right)=0 ;
$$

Sc.H2: $H\left(x, x^{\prime}\right)$ is symmetric in its arguments,

$$
H\left(x^{\prime}, x\right)=H\left(x, x^{\prime}\right)
$$

Living Reviews in Relativity

http: //www . livingreviews . org/lrr-2004-6 
Sc.H3: $H\left(x, x^{\prime}\right)$ agrees with the retarded Green's function if $x$ is in the chronological future of $x^{\prime}$

$$
H\left(x, x^{\prime}\right)=G_{+}\left(x, x^{\prime}\right) \quad \text { when } x \in I^{+}\left(x^{\prime}\right) ;
$$

Sc.H4: $H\left(x, x^{\prime}\right)$ agrees with the advanced Green's function if $x$ is in the chronological past of $x^{\prime}$

$$
H\left(x, x^{\prime}\right)=G_{-}\left(x, x^{\prime}\right) \quad \text { when } x \in I^{-}\left(x^{\prime}\right) .
$$

With a biscalar $H\left(x, x^{\prime}\right)$ satisfying these relations, a singular Green's function defined by

$$
G_{\mathrm{S}}\left(x, x^{\prime}\right)=\frac{1}{2}\left[G_{+}\left(x, x^{\prime}\right)+G_{-}\left(x, x^{\prime}\right)-H\left(x, x^{\prime}\right)\right]
$$

will satisfy all the properties listed previously: Property Sc.S1 comes as a consequence of Property Sc.H1 and the fact that both the advanced and the retarded Green's functions are solutions to the inhomogeneous wave equation, Property Sc.S2 follows directly from Property Sc.H2 and the definition of Equation (295), and Property Sc.S3 comes as a consequence of Properties Sc.H3, Property Sc.H4 and the properties of the retarded and advanced Green's functions.

The question is now: Does such a function $H\left(x, x^{\prime}\right)$ exist? I will present a plausibility argument for an affirmative answer. Later in this section we will see that $H\left(x, x^{\prime}\right)$ is guaranteed to exist in the local convex neighbourhood of $x^{\prime}$, where it is equal to $V\left(x, x^{\prime}\right)$. And in Section 4.3.6 we will see that there exist particular spacetimes for which $H\left(x, x^{\prime}\right)$ can be defined globally.

To satisfy all of Properties Sc.H4, Sc.H2, Sc.H3, and Sc.H4 might seem a tall order, but it should be possible. We first note that Property Sc.H4 is not independent from the rest: It follows from Property Sc.H2, Property Sc.H3, and the reciprocity relation (280) satisfied by the retarded and advanced Green's functions. Let $x \in I^{-}\left(x^{\prime}\right)$, so that $x^{\prime} \in I^{+}(x)$. Then $H\left(x, x^{\prime}\right)=H\left(x^{\prime}, x\right)$ by Property Sc.H2, and by Property Sc.H3 this is equal to $G_{+}\left(x^{\prime}, x\right)$. But by the reciprocity relation this is also equal to $G_{-}\left(x, x^{\prime}\right)$, and we have obtained Property Sc.H4. Alternatively, and this shall be our point of view in the next paragraph, we can think of Property Sc.H3 as following from Properties Sc.H2 and Sc.H4.

Because $H\left(x, x^{\prime}\right)$ satisfies the homogeneous wave equation (Property Sc.H1), it can be given the Kirkhoff representation of Equation (283): If $\Sigma$ is a spacelike hypersurface in the past of both $x$ and $x^{\prime}$, then

$$
H\left(x, x^{\prime}\right)=-\frac{1}{4 \pi} \int_{\Sigma}\left(G_{+}\left(x, x^{\prime \prime}\right) \nabla^{\alpha^{\prime \prime}} H\left(x^{\prime \prime}, x^{\prime}\right)-H\left(x^{\prime \prime}, x^{\prime}\right) \nabla^{\alpha^{\prime \prime}} G_{+}\left(x, x^{\prime \prime}\right)\right) d \Sigma_{\alpha^{\prime \prime}},
$$

where $d \Sigma_{\alpha^{\prime \prime}}$ is a surface element on $\Sigma$. The hypersurface can be partitioned into two segments, $\Sigma^{-}\left(x^{\prime}\right)$ and $\Sigma-\Sigma^{-}\left(x^{\prime}\right)$, with $\Sigma^{-}\left(x^{\prime}\right)$ denoting the intersection of $\Sigma$ with $I^{-}\left(x^{\prime}\right)$. To enforce Property Sc.H4 it suffices to choose for $H\left(x, x^{\prime}\right)$ initial data on $\Sigma^{-}\left(x^{\prime}\right)$ that agree with the initial data for the advanced Green's function; because both functions satisfy the homogeneous wave equation in $I^{-}\left(x^{\prime}\right)$, the agreement will be preserved in all of the domain of dependence of $\Sigma^{-}\left(x^{\prime}\right)$. The data on $\Sigma-\Sigma^{-}\left(x^{\prime}\right)$ is still free, and it should be possible to choose it so as to make $H\left(x, x^{\prime}\right)$ symmetric. Assuming that this can be done, we see that Property $\mathrm{Sc} . \mathrm{H} 2$ is enforced and we conclude that the Properties Sc.H1, Sc.H2, Sc.H3, and Sc.H4 can all be satisfied.

When $x$ is restricted to the normal convex neighbourhood of $x^{\prime}$, Properties Sc.H1, Sc.H2, Sc.H3, and Sc.H4 imply that

$$
H\left(x, x^{\prime}\right)=V\left(x, x^{\prime}\right)
$$

it should be stressed here that while $H\left(x, x^{\prime}\right)$ is assumed to be defined globally in the entire spacetime, the existence of $V\left(x, x^{\prime}\right)$ is limited to $\mathcal{N}\left(x^{\prime}\right)$. With Equations (269) and (295) we find that the singular Green's function is given explicitly by

$$
G_{\mathrm{S}}\left(x, x^{\prime}\right)=\frac{1}{2} U\left(x, x^{\prime}\right) \delta(\sigma)-\frac{1}{2} V\left(x, x^{\prime}\right) \theta(\sigma)
$$


in the normal convex neighbourhood. Equation (297) shows very clearly that the singular Green's function does not distinguish between past and future (Property Sc.S2), and that its support excludes $I^{ \pm}\left(x^{\prime}\right)$, in which $\theta(\sigma)=0$ (Property Sc.S3). From Equation (287) we get an analogous expression for the radiative Green's function:

$$
G_{\mathrm{R}}\left(x, x^{\prime}\right)=\frac{1}{2} U\left(x, x^{\prime}\right)\left[\delta_{+}(\sigma)-\delta_{-}(\sigma)\right]+V\left(x, x^{\prime}\right)\left[\theta_{+}(-\sigma)+\frac{1}{2} \theta(\sigma)\right] .
$$

This reveals directly that the radiative Green's function coincides with $G_{+}\left(x, x^{\prime}\right)$ in $I^{+}\left(x^{\prime}\right)$, in which $\theta(\sigma)=0$ and $\theta_{+}(-\sigma)=1$ (Property Sc.R2), and that its support does not include $I^{-}\left(x^{\prime}\right)$, in which $\theta(\sigma)=\theta_{+}(-\sigma)=0$ (Property Sc.R3).

\subsubsection{Example: Cosmological Green's functions}

To illustrate the general theory outlined in the previous Sections 4.3.1, 4.3.2, 4.3.3, 4.3.4, and 4.3.5, we consider here the specific case of a minimally-coupled $(\xi=0)$ scalar field in a cosmological spacetime with metric

$$
d s^{2}=a^{2}(\eta)\left(-d \eta^{2}+d x^{2}+d y^{2}+d z^{2}\right),
$$

where $a(\eta)$ is the scale factor expressed in terms of conformal time. For concreteness we take the universe to be matter dominated, so that $a(\eta)=C \eta^{2}$, where $C$ is a constant. This spacetime is one of the very few for which Green's functions can be explicitly constructed. The calculation presented here was first carried out by Burko, Harte, and Poisson [15]; it can be extended to other cosmologies.

To solve Green's equation $\square G\left(x, x^{\prime}\right)=-4 \pi \delta_{4}\left(x, x^{\prime}\right)$ we first introduce a reduced Green's function $g\left(x, x^{\prime}\right)$ defined by

$$
G\left(x, x^{\prime}\right)=\frac{g\left(x, x^{\prime}\right)}{a(\eta) a\left(\eta^{\prime}\right)}
$$

Substitution yields

$$
\left(-\frac{\partial^{2}}{\partial \eta^{2}}+\nabla^{2}+\frac{2}{\eta^{2}}\right) g\left(x, x^{\prime}\right)=-4 \pi \delta\left(\eta-\eta^{\prime}\right) \delta_{3}\left(\boldsymbol{x}-\boldsymbol{x}^{\prime}\right)
$$

where $\boldsymbol{x}=(x, y, z)$ is a vector in three-dimensional flat space, and $\nabla^{2}$ is the Laplacian operator in this space. We next expand $g\left(x, x^{\prime}\right)$ in terms of plane-wave solutions to Laplace's equation,

$$
g\left(x, x^{\prime}\right)=\frac{1}{(2 \pi)^{3}} \int \tilde{g}\left(\eta, \eta^{\prime} ; \boldsymbol{k}\right) e^{i \boldsymbol{k} \cdot\left(\boldsymbol{x}-\boldsymbol{x}^{\prime}\right)} d^{3} k
$$

and we substitute this back into Equation (301). The result, after also Fourier transforming $\delta_{3}\left(\boldsymbol{x}-\boldsymbol{x}^{\prime}\right)$, is an ordinary differential equation for $\tilde{g}\left(\eta, \eta^{\prime} ; \boldsymbol{k}\right)$,

$$
\left(\frac{d^{2}}{d \eta^{2}}+k^{2}-\frac{2}{\eta^{2}}\right) \tilde{g}=4 \pi \delta\left(\eta-\eta^{\prime}\right)
$$

where $k^{2}=\boldsymbol{k} \cdot \boldsymbol{k}$. To generate the retarded Green's function we set

$$
\tilde{g}_{+}\left(\eta, \eta^{\prime} ; \boldsymbol{k}\right)=\theta\left(\eta-\eta^{\prime}\right) \hat{g}\left(\eta, \eta^{\prime} ; k\right),
$$

in which we indicate that $\hat{g}$ depends only on the modulus of the vector $\boldsymbol{k}$. To generate the advanced Green's function we would set instead $\tilde{g}_{-}\left(\eta, \eta^{\prime} ; \boldsymbol{k}\right)=\theta\left(\eta^{\prime}-\eta\right) \hat{g}\left(\eta, \eta^{\prime} ; k\right)$. The following manipulations will refer specifically to the retarded Green's function; they are easily adapted to the case of the advanced Green's function.

Living Reviews in Relativity

http://www. livingreviews . org//rr-2004-6 
Substitution of Equation (304) into Equation (303) reveals that $\hat{g}$ must satisfy the homogeneous equation

$$
\left(\frac{d^{2}}{d \eta^{2}}+k^{2}-\frac{2}{\eta^{2}}\right) \hat{g}=0
$$

together with the boundary conditions

$$
\hat{g}\left(\eta=\eta^{\prime} ; k\right)=0, \quad \frac{d \hat{g}}{d \eta}\left(\eta=\eta^{\prime} ; k\right)=4 \pi .
$$

Inserting Equation (304) into Equation (302) and integrating over the angular variables associated with the vector $\boldsymbol{k}$ yields

$$
g_{+}\left(x, x^{\prime}\right)=\frac{\theta(\Delta \eta)}{2 \pi^{2} R} \int_{0}^{\infty} \hat{g}\left(\eta, \eta^{\prime} ; k\right) k \sin (k R) d k,
$$

where $\Delta \eta \equiv \eta-\eta^{\prime}$ and $R \equiv\left|\boldsymbol{x}-\boldsymbol{x}^{\prime}\right|$.

Equation (305) has $\cos (k \Delta \eta)-(k \eta)^{-1} \sin (k \Delta \eta)$ and $\sin (k \Delta \eta)+(k \eta)^{-1} \cos (k \Delta \eta)$ as linearly independent solutions, and $\hat{g}\left(\eta, \eta^{\prime} ; k\right)$ must be given by a linear superposition. The coefficients can be functions of $\eta^{\prime}$, and after imposing Equations (306) we find that the appropriate combination is

$$
\hat{g}\left(\eta, \eta^{\prime} ; k\right)=\frac{4 \pi}{k}\left[\left(1+\frac{1}{k^{2} \eta \eta^{\prime}}\right) \sin (k \Delta \eta)-\frac{\Delta \eta}{k \eta \eta^{\prime}} \cos (k \Delta \eta)\right] .
$$

Substituting this into Equation (307) and using the identity $(2 / \pi) \int_{0}^{\infty} \sin (\omega x) \sin \left(\omega x^{\prime}\right) d \omega=\delta(x-$ $\left.x^{\prime}\right)-\delta\left(x+x^{\prime}\right)$ yields

$$
g_{+}\left(x, x^{\prime}\right)=\frac{\delta(\Delta \eta-R)}{R}+\frac{\theta(\Delta \eta)}{\eta \eta^{\prime}} \frac{2}{\pi} \int_{0}^{\infty} \frac{1}{k} \sin (k \Delta \eta) \cos (k R) d k
$$

after integration by parts. The integral evaluates to $\theta(\Delta \eta-R)$.

We have arrived at

$$
g_{+}\left(x, x^{\prime}\right)=\frac{\delta\left(\eta-\eta^{\prime}-\left|\boldsymbol{x}-\boldsymbol{x}^{\prime}\right|\right)}{\left|\boldsymbol{x}-\boldsymbol{x}^{\prime}\right|}+\frac{\theta\left(\eta-\eta^{\prime}-\left|\boldsymbol{x}-\boldsymbol{x}^{\prime}\right|\right)}{\eta \eta^{\prime}}
$$

for our final expression for the retarded Green's function. The advanced Green's function is given instead by

$$
g_{-}\left(x, x^{\prime}\right)=\frac{\delta\left(\eta-\eta^{\prime}+\left|\boldsymbol{x}-\boldsymbol{x}^{\prime}\right|\right)}{\left|\boldsymbol{x}-\boldsymbol{x}^{\prime}\right|}+\frac{\theta\left(-\eta+\eta^{\prime}-\left|\boldsymbol{x}-\boldsymbol{x}^{\prime}\right|\right)}{\eta \eta^{\prime}} .
$$

The distributions $g_{ \pm}\left(x, x^{\prime}\right)$ are solutions to the reduced Green's equation of Equation (301). The actual Green's functions are obtained by substituting Equations (309) and (310) into Equation (300). We note that the support of the retarded Green's function is given by $\eta-\eta^{\prime} \geq\left|\boldsymbol{x}-\boldsymbol{x}^{\prime}\right|$, while the support of the advanced Green's function is given by $\eta-\eta^{\prime} \leq-\left|\boldsymbol{x}-\boldsymbol{x}^{\prime}\right|$.

It may be verified that the symmetric two-point function

$$
h\left(x, x^{\prime}\right)=\frac{1}{\eta \eta^{\prime}}
$$

satisfies all of the Properties Sc.H1, Sc.H2, Sc.H3, and Sc.H4 listed in Section 4.3.5; it may thus be used to define singular and radiative Green's functions. According to Equation (295) the singular Green's function is given by

$$
\begin{aligned}
g_{\mathrm{S}}\left(x, x^{\prime}\right)= & \frac{1}{2\left|\boldsymbol{x}-\boldsymbol{x}^{\prime}\right|}\left[\delta\left(\eta-\eta^{\prime}-\left|\boldsymbol{x}-\boldsymbol{x}^{\prime}\right|\right)+\delta\left(\eta-\eta^{\prime}+\left|\boldsymbol{x}-\boldsymbol{x}^{\prime}\right|\right)\right] \\
& +\frac{1}{2 \eta \eta^{\prime}}\left[\theta\left(\eta-\eta^{\prime}-\left|\boldsymbol{x}-\boldsymbol{x}^{\prime}\right|\right)-\theta\left(\eta-\eta^{\prime}+\left|\boldsymbol{x}-\boldsymbol{x}^{\prime}\right|\right)\right]
\end{aligned}
$$


and its support is limited to the interval $-\left|\boldsymbol{x}-\boldsymbol{x}^{\prime}\right| \leq \eta-\eta^{\prime} \leq\left|\boldsymbol{x}-\boldsymbol{x}^{\prime}\right|$. According to Equation (287) the radiative Green's function is given by

$$
\begin{aligned}
g_{\mathrm{R}}\left(x, x^{\prime}\right)= & \frac{1}{2\left|\boldsymbol{x}-\boldsymbol{x}^{\prime}\right|}\left[\delta\left(\eta-\eta^{\prime}-\left|\boldsymbol{x}-\boldsymbol{x}^{\prime}\right|\right)-\delta\left(\eta-\eta^{\prime}+\left|\boldsymbol{x}-\boldsymbol{x}^{\prime}\right|\right)\right] \\
& +\frac{1}{2 \eta \eta^{\prime}}\left[\theta\left(\eta-\eta^{\prime}-\left|\boldsymbol{x}-\boldsymbol{x}^{\prime}\right|\right)+\theta\left(\eta-\eta^{\prime}+\left|\boldsymbol{x}-\boldsymbol{x}^{\prime}\right|\right)\right]
\end{aligned}
$$

its support is given by $\eta-\eta^{\prime} \geq-\left|\boldsymbol{x}-\boldsymbol{x}^{\prime}\right|$, and for $\eta-\eta^{\prime} \geq\left|\boldsymbol{x}-\boldsymbol{x}^{\prime}\right|$ the radiative Green's function agrees with the retarded Green's function.

As a final observation we note that for this cosmological spacetime, the normal convex neighbourhood of any point $x$ consists of the whole spacetime manifold (which excludes the cosmological singularity at $a=0$ ). The Hadamard construction of the Green's functions is therefore valid globally, a fact that is immediately revealed by Equations (309) and (310).

\subsection{Electromagnetic Green's functions}

\subsubsection{Equations of electromagnetism}

The electromagnetic field tensor $F_{\alpha \beta}=\nabla_{\alpha} A_{\beta}-\nabla_{\beta} A_{\alpha}$ is expressed in terms of a vector potential $A_{\alpha}$. In the Lorenz gauge $\nabla_{\alpha} A^{\alpha}=0$, the vector potential satisfies the wave equation

$$
\square A^{\alpha}-R_{\beta}^{\alpha} A^{\beta}=-4 \pi j^{\alpha},
$$

where $\square=g^{\alpha \beta} \nabla_{\alpha} \nabla_{\beta}$ is the wave operator, $R_{\beta}^{\alpha}$ the Ricci tensor, and $j^{\alpha}$ a prescribed current density. The wave equation enforces the condition $\nabla_{\alpha} j^{\alpha}=0$, which expresses charge conservation.

The solution to the wave equation is written as

$$
A^{\alpha}(x)=\int G_{\beta^{\prime}}^{\alpha}\left(x, x^{\prime}\right) j^{\beta^{\prime}}\left(x^{\prime}\right) \sqrt{-g^{\prime}} d^{4} x^{\prime},
$$

in terms of a Green's function $G^{\alpha}{ }_{\beta^{\prime}}\left(x, x^{\prime}\right)$ that satisfies

$$
\square G_{\beta^{\prime}}^{\alpha}\left(x, x^{\prime}\right)-R_{\beta}^{\alpha}(x) G_{\beta^{\prime}}^{\beta}\left(x, x^{\prime}\right)=-4 \pi g_{\beta^{\prime}}^{\alpha}\left(x, x^{\prime}\right) \delta_{4}\left(x, x^{\prime}\right),
$$

where $g_{\beta^{\prime}}^{\alpha}\left(x, x^{\prime}\right)$ is a parallel propagator and $\delta_{4}\left(x, x^{\prime}\right)$ an invariant Dirac distribution. The parallel propagator is inserted on the right-hand side of Equation (316) to keep the index structure of the equation consistent from side to side; because $g_{\beta^{\prime}}^{\alpha}\left(x, x^{\prime}\right) \delta_{4}\left(x, x^{\prime}\right)$ is distributionally equal to $\left[g_{\beta^{\prime}}^{\alpha}\right] \delta_{4}\left(x, x^{\prime}\right)=\delta_{\beta^{\prime}}^{\alpha^{\prime}} \delta_{4}\left(x, x^{\prime}\right)$, it could have been replaced by either $\delta_{\beta^{\prime}}^{\alpha^{\prime}}$ or $\delta_{\beta}^{\alpha}$. It is easy to check that by virtue of Equation (316), the vector potential of Equation (315) satisfies the wave equation of Equation (314).

We will assume that the retarded Green's function $G_{+\beta^{\prime}}^{\alpha}\left(x, x^{\prime}\right)$, which is nonzero if $x$ is in the causal future of $x^{\prime}$, and the advanced Green's function $G_{-\beta^{\prime}}^{\alpha}\left(x, x^{\prime}\right)$, which is nonzero if $x$ is in the causal past of $x^{\prime}$, exist as distributions and can be defined globally in the entire spacetime.

\subsubsection{Hadamard construction of the Green's functions}

Assuming throughout this section that $x$ is in the normal convex neighbourhood of $x^{\prime}$, we make the ansatz

$$
G_{ \pm \beta^{\prime}}^{\alpha}\left(x, x^{\prime}\right)=U_{\beta^{\prime}}^{\alpha}\left(x, x^{\prime}\right) \delta_{ \pm}(\sigma)+V_{\beta^{\prime}}^{\alpha}\left(x, x^{\prime}\right) \theta_{ \pm}(-\sigma),
$$

where $\theta_{ \pm}(-\sigma), \delta_{ \pm}(\sigma)$ are the light-cone distributions introduced in Section 4.2.2, and where $U_{\beta^{\prime}}^{\alpha}\left(x, x^{\prime}\right), V_{\beta^{\prime}}^{\alpha}\left(x, x^{\prime}\right)$ are smooth bitensors.

Living Reviews in Relativity

http: //www . livingreviews . org/lrr-2004-6 
To conveniently manipulate the Green's functions we shift $\sigma$ by a small positive quantity $\epsilon$. The Green's functions are then recovered by the taking the limit of

$$
G_{ \pm \beta^{\prime}}^{\epsilon \alpha}\left(x, x^{\prime}\right) \equiv U_{\beta^{\prime}}^{\alpha}\left(x, x^{\prime}\right) \delta_{ \pm}(\sigma+\epsilon)+V_{\beta^{\prime}}^{\alpha}\left(x, x^{\prime}\right) \theta_{ \pm}(-\sigma-\epsilon)
$$

as $\epsilon \rightarrow 0^{+}$. When we substitute this into the left-hand side of Equation (316) and then take the limit, we obtain

$$
\begin{aligned}
\square G_{ \pm \beta^{\prime}}^{\alpha}-R_{\beta}^{\alpha} G_{ \pm \beta^{\prime}}^{\beta}= & -4 \pi \delta_{4}\left(x, x^{\prime}\right) U_{\beta^{\prime}}^{\alpha}+\delta_{ \pm}^{\prime}(\sigma)\left\{2 U_{\beta^{\prime} ; \gamma}^{\alpha} \sigma^{\gamma}+\left(\sigma_{\gamma}^{\gamma}-4\right) U_{\beta^{\prime}}^{\alpha}\right\} \\
& +\delta_{ \pm}(\sigma)\left\{-2 V_{\beta^{\prime} ; \gamma}^{\alpha} \sigma^{\gamma}+\left(2-\sigma_{\gamma}^{\gamma}\right) V_{\beta^{\prime}}^{\alpha}+\square U_{\beta^{\prime}}^{\alpha}-R_{\beta}^{\alpha} U_{\beta^{\prime}}^{\beta}\right\} \\
& +\theta_{ \pm}(-\sigma)\left\{\square V_{\beta^{\prime}}^{\alpha}-R_{\beta}^{\alpha} V_{\beta^{\prime}}^{\beta}\right\}
\end{aligned}
$$

after a routine computation similar to the one presented at the beginning of Section 4.3.2. Comparison with Equation (316) returns

- the equations

$$
\left[U_{\beta^{\prime}}^{\alpha}\right]=\left[g_{\beta^{\prime}}^{\alpha}\right]=\delta_{\beta^{\prime}}^{\alpha^{\prime}}
$$

and

$$
2 U_{\beta^{\prime} ; \gamma}^{\alpha} \sigma^{\gamma}+\left(\sigma_{\gamma}^{\gamma}-4\right) U_{\beta^{\prime}}^{\alpha}=0
$$

that determine $U_{\beta^{\prime}}^{\alpha}\left(x, x^{\prime}\right)$;

- the equation

$$
\check{V}_{\beta^{\prime} ; \gamma}^{\alpha} \sigma^{\gamma}+\frac{1}{2}\left(\sigma_{\gamma}^{\gamma}-2\right) \check{V}_{\beta^{\prime}}^{\alpha}=\left.\frac{1}{2}\left(\square U_{\beta^{\prime}}^{\alpha}-R_{\beta}^{\alpha} U_{\beta^{\prime}}^{\beta}\right)\right|_{\sigma=0}
$$

that determines $\check{V}_{\beta^{\prime}}^{\alpha}\left(x, x^{\prime}\right)$, the restriction of $V_{\beta^{\prime}}^{\alpha}\left(x, x^{\prime}\right)$ on the light cone $\sigma\left(x, x^{\prime}\right)=0$; and

- the wave equation

$$
\square V_{\beta^{\prime}}^{\alpha}-R_{\beta}^{\alpha} V_{\beta^{\prime}}^{\beta}=0
$$

that determines $V_{\beta^{\prime}}^{\alpha}\left(x, x^{\prime}\right)$ inside the light cone.

Equation (319) can be integrated along the unique geodesic $\beta$ that links $x^{\prime}$ to $x$. The initial conditions are provided by Equation (318), and if we set $U_{\beta^{\prime}}^{\alpha}\left(x, x^{\prime}\right)=g_{\beta^{\prime}}^{\alpha}\left(x, x^{\prime}\right) U\left(x, x^{\prime}\right)$, we find that these equations reduce to Equations (272) and (271), respectively. According to Equation (273), then, we have

$$
U_{\beta^{\prime}}^{\alpha}\left(x, x^{\prime}\right)=g_{\beta^{\prime}}^{\alpha}\left(x, x^{\prime}\right) \Delta^{1 / 2}\left(x, x^{\prime}\right),
$$

which reduces to

$$
U_{\beta^{\prime}}^{\alpha}=g_{\beta^{\prime}}^{\alpha}\left(1+\frac{1}{12} R_{\gamma^{\prime} \delta^{\prime}} \sigma^{\gamma^{\prime}} \sigma^{\delta^{\prime}}+\mathcal{O}\left(\lambda^{3}\right)\right)
$$

near coincidence, with $\lambda$ denoting the affine-parameter distance between $x^{\prime}$ and $x$. Differentiation of this relation gives

$$
\begin{aligned}
U_{\beta^{\prime} ; \gamma}^{\alpha} & =\frac{1}{2} g_{\gamma}^{\gamma^{\prime}}\left(g_{\alpha^{\prime}}^{\alpha} R_{\beta^{\prime} \gamma^{\prime} \delta^{\prime}}^{\alpha^{\prime}}-\frac{1}{3} g_{\beta^{\prime}}^{\alpha} R_{\gamma^{\prime} \delta^{\prime}}\right) \sigma^{\delta^{\prime}}+\mathcal{O}\left(\lambda^{2}\right), \\
U_{\beta^{\prime} ; \gamma^{\prime}}^{\alpha} & =\frac{1}{2}\left(g_{\alpha^{\prime}}^{\alpha} R_{\beta^{\prime} \gamma^{\prime} \delta^{\prime}}^{\alpha^{\prime}}+\frac{1}{3} g_{\beta^{\prime}}^{\alpha} R_{\gamma^{\prime} \delta^{\prime}}\right) \sigma^{\delta^{\prime}}+\mathcal{O}\left(\lambda^{2}\right),
\end{aligned}
$$

and eventually,

$$
\left[\square U_{\beta^{\prime}}^{\alpha}\right]=\frac{1}{6} \delta_{\beta^{\prime}}^{\alpha^{\prime}} R\left(x^{\prime}\right) .
$$


Similarly, Equation (320) can be integrated along each null geodesic that generates the null cone $\sigma\left(x, x^{\prime}\right)=0$. The initial values are obtained by taking the coincidence limit of this equation, using Equations (318), (326), and the additional relation $\left[\sigma_{\gamma}^{\gamma}\right]=4$. We arrive at

$$
\left[V_{\beta^{\prime}}^{\alpha}\right]=-\frac{1}{2}\left(R_{\beta^{\prime}}^{\alpha^{\prime}}-\frac{1}{6} \delta_{\beta^{\prime}}^{\alpha^{\prime}} R^{\prime}\right)
$$

With the characteristic data obtained by integrating Equation (320), the wave equation of Equation (321) admits a unique solution.

To summarize, the retarded and advanced electromagnetic Green's functions are given by Equation (317) with $U_{\beta^{\prime}}^{\alpha}\left(x, x^{\prime}\right)$ given by Equation (322) and $V_{\beta^{\prime}}^{\alpha}\left(x, x^{\prime}\right)$ determined by Equation (321) and the characteristic data constructed with Equations (320) and (327). It should be emphasized that the construction provided in this section is restricted to $\mathcal{N}\left(x^{\prime}\right)$, the normal convex neighbourhood of the reference point $x^{\prime}$.

\subsubsection{Reciprocity and Kirchhoff representation}

Like their scalar counterparts, the (globally defined) electromagnetic Green's functions satisfy a reciprocity relation, the statement of which is

$$
G_{\beta^{\prime} \alpha}^{-}\left(x^{\prime}, x\right)=G_{\alpha \beta^{\prime}}^{+}\left(x, x^{\prime}\right)
$$

The derivation of Equation (328) is virtually identical to what was presented in Section 4.3.3, and we shall not present the details. It suffices to mention that it is based on the identities

$$
G_{\alpha \beta^{\prime}}^{+}\left(x, x^{\prime}\right)\left(\square G_{-\gamma^{\prime \prime}}^{\alpha}\left(x, x^{\prime \prime}\right)-R_{\gamma}^{\alpha} G_{-\gamma^{\prime \prime}}^{\gamma}\left(x, x^{\prime \prime}\right)\right)=-4 \pi G_{\alpha \beta^{\prime}}^{+}\left(x, x^{\prime}\right) g_{\gamma^{\prime \prime}}^{\alpha}\left(x, x^{\prime \prime}\right) \delta_{4}\left(x, x^{\prime \prime}\right)
$$

and

$$
G_{\alpha \gamma^{\prime \prime}}^{-}\left(x, x^{\prime \prime}\right)\left(\square G_{+\beta^{\prime}}^{\alpha}\left(x, x^{\prime}\right)-R_{\gamma}^{\alpha} G_{+\beta^{\prime}}^{\gamma}\left(x, x^{\prime}\right)\right)=-4 \pi G_{\alpha \gamma^{\prime \prime}}^{-}\left(x, x^{\prime \prime}\right) g_{\beta^{\prime}}^{\alpha}\left(x, x^{\prime}\right) \delta_{4}\left(x, x^{\prime}\right)
$$

A direct consequence of the reciprocity relation is

$$
V_{\beta^{\prime} \alpha}\left(x^{\prime}, x\right)=V_{\alpha \beta^{\prime}}\left(x, x^{\prime}\right),
$$

the statement that the bitensor $V_{\alpha \beta^{\prime}}\left(x, x^{\prime}\right)$ is symmetric in its indices and arguments.

The Kirchhoff representation for the electromagnetic vector potential is formulated as follows. Suppose that $A^{\alpha}(x)$ satisfies the homogeneous version of Equation (314) and that initial values $A^{\alpha^{\prime}}\left(x^{\prime}\right), n^{\beta^{\prime}} \nabla_{\beta^{\prime}} A^{\alpha^{\prime}}\left(x^{\prime}\right)$ are specified on a spacelike hypersurface $\Sigma$. Then the value of the potential at a point $x$ in the future of $\Sigma$ is given by

$$
A^{\alpha}(x)=-\frac{1}{4 \pi} \int_{\Sigma}\left(G_{+\beta^{\prime}}^{\alpha}\left(x, x^{\prime}\right) \nabla^{\gamma^{\prime}} A^{\beta^{\prime}}\left(x^{\prime}\right)-A^{\beta^{\prime}}\left(x^{\prime}\right) \nabla^{\gamma^{\prime}} G_{+\beta^{\prime}}^{\alpha}\left(x, x^{\prime}\right)\right) d \Sigma_{\gamma^{\prime}},
$$

where $d \Sigma_{\gamma^{\prime}}=-n_{\gamma^{\prime}} d V$ is a surface element on $\Sigma ; n_{\gamma^{\prime}}$ is the future-directed unit normal and $d V$ is the invariant volume element on the hypersurface. The derivation of Equation (330) is virtually identical to what was presented in Section 4.3.4.

\subsubsection{Singular and radiative Green's functions}

We shall now construct singular and radiative Green's functions for the electromagnetic field. The treatment here parallels closely what was presented in Section 4.3.5, and the reader is referred to that section for a more complete discussion.

Living Reviews in Relativity

http://www. livingreviews.org//rr-2004-6 
We begin by introducing the bitensor $H_{\beta^{\prime}}^{\alpha}\left(x, x^{\prime}\right)$ with properties

Em.H1: $H_{\beta^{\prime}}^{\alpha}\left(x, x^{\prime}\right)$ satisfies the homogeneous wave equation,

$$
\square H_{\beta^{\prime}}^{\alpha}\left(x, x^{\prime}\right)-R_{\beta}^{\alpha}(x) H_{\beta^{\prime}}^{\beta}\left(x, x^{\prime}\right)=0 ;
$$

Em.H2: $H_{\beta^{\prime}}^{\alpha}\left(x, x^{\prime}\right)$ is symmetric in its indices and arguments,

$$
H_{\beta^{\prime} \alpha}\left(x^{\prime}, x\right)=H_{\alpha \beta^{\prime}}\left(x, x^{\prime}\right)
$$

Em.H3: $H_{\beta^{\prime}}^{\alpha}\left(x, x^{\prime}\right)$ agrees with the retarded Green's function if $x$ is in the chronological future of $x^{\prime}$,

$$
H_{\beta^{\prime}}^{\alpha}\left(x, x^{\prime}\right)=G_{+\beta^{\prime}}^{\alpha}\left(x, x^{\prime}\right) \quad \text { when } x \in I^{+}\left(x^{\prime}\right) ;
$$

Em.H4: $H_{\beta^{\prime}}^{\alpha}\left(x, x^{\prime}\right)$ agrees with the advanced Green's function if $x$ is in the chronological past of $x^{\prime}$,

$$
H_{\beta^{\prime}}^{\alpha}\left(x, x^{\prime}\right)=G_{-\beta^{\prime}}^{\alpha}\left(x, x^{\prime}\right) \quad \text { when } x \in I^{-}\left(x^{\prime}\right) .
$$

It is easy to prove that Property Em.H4 follows from Property Em.H2, Property Em.H3, and the reciprocity relation (328) satisfied by the retarded and advanced Green's functions. That such a bitensor exists can be argued along the same lines as those presented in Section 4.3.5.

Equipped with the bitensor $H_{\beta^{\prime}}^{\alpha}\left(x, x^{\prime}\right)$ we define the singular Green's function to be

$$
G_{\mathrm{S} \beta^{\prime}}^{\alpha}\left(x, x^{\prime}\right)=\frac{1}{2}\left[G_{+\beta^{\prime}}^{\alpha}\left(x, x^{\prime}\right)+G_{-\beta^{\prime}}^{\alpha}\left(x, x^{\prime}\right)-H_{\beta^{\prime}}^{\alpha}\left(x, x^{\prime}\right)\right] .
$$

This comes with the properties

Em.S1: $G_{\mathrm{S} \beta^{\prime}}^{\alpha}\left(x, x^{\prime}\right)$ satisfies the inhomogeneous wave equation,

$$
\square G_{\mathrm{S} \beta^{\prime}}^{\alpha}\left(x, x^{\prime}\right)-R_{\beta}^{\alpha}(x) G_{\mathrm{S}^{\prime}}^{\beta}\left(x, x^{\prime}\right)=-4 \pi g_{\beta^{\prime}}^{\alpha}\left(x, x^{\prime}\right) \delta_{4}\left(x, x^{\prime}\right) ;
$$

Em.S2: $G_{\mathrm{S}^{\prime}}^{\alpha}\left(x, x^{\prime}\right)$ is symmetric in its indices and arguments,

$$
G_{\beta^{\prime} \alpha}^{\mathrm{S}}\left(x^{\prime}, x\right)=G_{\alpha \beta^{\prime}}^{\mathrm{S}}\left(x, x^{\prime}\right) ;
$$

Em.S3: $G_{\mathrm{S} \beta^{\prime}}^{\alpha}\left(x, x^{\prime}\right)$ vanishes if $x$ is in the chronological past or future of $x^{\prime}$,

$$
G_{\mathrm{S} \beta^{\prime}}^{\alpha}\left(x, x^{\prime}\right)=0 \quad \text { when } x \in I^{ \pm}\left(x^{\prime}\right) .
$$

These can be established as consequences of Properties Em.H1, Em.H2, Em.H3, and Em.H4, and the properties of the retarded and advanced Green's functions.

The radiative Green's function is then defined by

$$
G_{\mathrm{R} \beta^{\prime}}^{\alpha}\left(x, x^{\prime}\right)=G_{+\beta^{\prime}}^{\alpha}\left(x, x^{\prime}\right)-G_{\mathrm{S} \beta^{\prime}}^{\alpha}\left(x, x^{\prime}\right),
$$

and it comes with the properties

Em.R1: $G_{\mathrm{R} \beta^{\prime}}^{\alpha}\left(x, x^{\prime}\right)$ satisfies the homogeneous wave equation,

$$
\square G_{\mathrm{R} \beta^{\prime}}^{\alpha}\left(x, x^{\prime}\right)-R_{\beta}^{\alpha}(x) G_{\mathrm{R} \beta^{\prime}}^{\beta}\left(x, x^{\prime}\right)=0 ;
$$



Em.R2: $G_{\mathrm{R} \beta^{\prime}}^{\alpha}\left(x, x^{\prime}\right)$ agrees with the retarded Green's function if $x$ is in the chronological future
of $x^{\prime}$

$$
G_{\mathrm{R} \beta^{\prime}}^{\alpha}\left(x, x^{\prime}\right)=G_{+\beta^{\prime}}^{\alpha}\left(x, x^{\prime}\right) \quad \text { when } x \in I^{+}\left(x^{\prime}\right)
$$

Em.R3: $G_{\mathrm{R} \beta^{\prime}}^{\alpha}\left(x, x^{\prime}\right)$ vanishes if $x$ is in the chronological past of $x^{\prime}$,

$$
G_{\mathrm{R} \beta^{\prime}}^{\alpha}\left(x, x^{\prime}\right)=0 \quad \text { when } x \in I^{-}\left(x^{\prime}\right) .
$$

Those follow immediately from Properties Em.S1, Em.S2, and Em.S3 and the properties of the retarded Green's function.

When $x$ is restricted to the normal convex neighbourhood of $x^{\prime}$, we have the explicit relations

$$
\begin{aligned}
H_{\beta^{\prime}}^{\alpha}\left(x, x^{\prime}\right) & =V_{\beta^{\prime}}^{\alpha}\left(x, x^{\prime}\right), \\
G_{\mathrm{S} \beta^{\prime}}^{\alpha}\left(x, x^{\prime}\right) & =\frac{1}{2} U_{\beta^{\prime}}^{\alpha}\left(x, x^{\prime}\right) \delta(\sigma)-\frac{1}{2} V_{\beta^{\prime}}^{\alpha}\left(x, x^{\prime}\right) \theta(\sigma), \\
G_{\mathrm{R} \beta^{\prime}}^{\alpha}\left(x, x^{\prime}\right) & =\frac{1}{2} U_{\beta^{\prime}}^{\alpha}\left(x, x^{\prime}\right)\left[\delta_{+}(\sigma)-\delta_{-}(\sigma)\right]+V_{\beta^{\prime}}^{\alpha}\left(x, x^{\prime}\right)\left[\theta_{+}(-\sigma)+\frac{1}{2} \theta(\sigma)\right] .
\end{aligned}
$$

From these we see clearly that the singular Green's function does not distinguish between past and future (Property Em.S2), and that its support excludes $I^{ \pm}\left(x^{\prime}\right)$ (Property Em.S3). We see also that the radiative Green's function coincides with $G_{+\beta^{\prime}}^{\alpha}\left(x, x^{\prime}\right)$ in $I^{+}\left(x^{\prime}\right)$ (Property Em.R2), and that its support does not include $I^{-}\left(x^{\prime}\right)$ (Property Em.R3).

\subsection{Gravitational Green's functions}

\subsubsection{Equations of linearized gravity}

We are given a background spacetime for which the metric $g_{\alpha \beta}$ satisfies the Einstein field equations in vacuum. We then perturb the metric from $g_{\alpha \beta}$ to

$$
\mathrm{g}_{\alpha \beta}=g_{\alpha \beta}+h_{\alpha \beta} \text {. }
$$

The metric perturbation $h_{\alpha \beta}$ is assumed to be small, and when working out the Einstein field equations to be satisfied by the new metric $\mathrm{g}_{\alpha \beta}$, we work consistently to first order in $h_{\alpha \beta}$. To simplify the expressions we use the trace-reversed potentials $\gamma_{\alpha \beta}$ defined by

$$
\gamma_{\alpha \beta}=h_{\alpha \beta}-\frac{1}{2}\left(g^{\gamma \delta} h_{\gamma \delta}\right) g_{\alpha \beta}
$$

and we impose the Lorenz gauge condition,

$$
\gamma_{; \beta}^{\alpha \beta}=0
$$

In this equation, and in all others below, indices are raised and lowered with the background metric $g_{\alpha \beta}$. Similarly, the connection involved in Equation (348), and in all other equations below, is the one that is compatible with the background metric. If $T^{\alpha \beta}$ is the perturbing stress-energy tensor, then by virtue of the linearized Einstein field equations the perturbation field obeys the wave equation

$$
\square \gamma^{\alpha \beta}+2 R_{\gamma \delta}^{\alpha \beta} \gamma^{\gamma \delta}=-16 \pi T^{\alpha \beta},
$$

in which $\square=g^{\alpha \beta} \nabla_{\alpha} \nabla_{\beta}$ is the wave operator and $R_{\gamma \alpha \delta \beta}$ the Riemann tensor. In first-order perturbation theory, the stress-energy tensor must be conserved in the background spacetime: $T_{; \beta}^{\alpha \beta}=0$.

Living Reviews in Relativity

http: //www . livingreviews . org//rr-2004-6 
The solution to the wave equation is written as

$$
\gamma^{\alpha \beta}(x)=4 \int G_{\gamma^{\prime} \delta^{\prime}}^{\alpha \beta}\left(x, x^{\prime}\right) T^{\gamma^{\prime} \delta^{\prime}}\left(x^{\prime}\right) \sqrt{-g^{\prime}} d^{4} x^{\prime},
$$

in terms of a Green's function $G_{\gamma^{\prime} \delta^{\prime}}^{\alpha \beta}\left(x, x^{\prime}\right)$ that satisfies [53]

$$
\square G_{\gamma^{\prime} \delta^{\prime}}^{\alpha \beta}\left(x, x^{\prime}\right)+2 R_{\gamma \delta}^{\alpha \beta}(x) G_{\gamma^{\prime} \delta^{\prime}}^{\gamma \delta}\left(x, x^{\prime}\right)=-4 \pi g_{\gamma^{\prime}}^{(\alpha}\left(x, x^{\prime}\right) g_{\delta^{\prime}}^{\beta)}\left(x, x^{\prime}\right) \delta_{4}\left(x, x^{\prime}\right),
$$

where $g_{\gamma^{\prime}}^{\alpha}\left(x, x^{\prime}\right)$ is a parallel propagator and $\delta_{4}\left(x, x^{\prime}\right)$ an invariant Dirac functional. The parallel propagators are inserted on the right-hand side of Equation (351) to keep the index structure of the equation consistent from side to side; in particular, both sides of the equation are symmetric in $\alpha$ and $\beta$, and in $\gamma^{\prime}$ and $\delta^{\prime}$. It is easy to check that by virtue of Equation (351), the perturbation field of Equation (350) satisfies the wave equation of Equation (349). Once $\gamma_{\alpha \beta}$ is known, the metric perturbation can be reconstructed from the relation $h_{\alpha \beta}=\gamma_{\alpha \beta}-\frac{1}{2}\left(g^{\gamma \delta} \gamma_{\gamma \delta}\right) g_{\alpha \beta}$.

We will assume that the retarded Green's function $G_{+\gamma^{\prime} \delta^{\prime}}^{\alpha \beta}\left(x, x^{\prime}\right)$, which is nonzero if $x$ is in the causal future of $x^{\prime}$, and the advanced Green's function $G_{-\gamma^{\prime} \delta^{\prime}}^{\alpha \beta}\left(x, x^{\prime}\right)$, which is nonzero if $x$ is in the causal past of $x^{\prime}$, exist as distributions and can be defined globally in the entire background spacetime.

\subsubsection{Hadamard construction of the Green's functions}

Assuming throughout this section that $x$ is in the normal convex neighbourhood of $x^{\prime}$, we make the ansatz

$$
G_{ \pm \gamma^{\prime} \delta^{\prime}}^{\alpha \beta}\left(x, x^{\prime}\right)=U_{\gamma^{\prime} \delta^{\prime}}^{\alpha \beta}\left(x, x^{\prime}\right) \delta_{ \pm}(\sigma)+V_{\gamma^{\prime} \delta^{\prime}}^{\alpha \beta}\left(x, x^{\prime}\right) \theta_{ \pm}(-\sigma)
$$

where $\theta_{ \pm}(-\sigma), \delta_{ \pm}(\sigma)$ are the light-cone distributions introduced in Section 4.2.2, and where $U_{\gamma^{\prime} \delta^{\prime}}^{\alpha \beta}\left(x, x^{\prime}\right), V_{\gamma^{\prime} \delta^{\prime}}^{\alpha \beta}\left(x, x^{\prime}\right)$ are smooth bitensors.

To conveniently manipulate the Green's functions we shift $\sigma$ by a small positive quantity $\epsilon$. The Green's functions are then recovered by the taking the limit of

$$
G_{ \pm}^{\epsilon \beta} \gamma_{\gamma^{\prime} \delta^{\prime}}\left(x, x^{\prime}\right)=U_{\gamma^{\prime} \delta^{\prime}}^{\alpha \beta}\left(x, x^{\prime}\right) \delta_{ \pm}(\sigma+\epsilon)+V_{\gamma^{\prime} \delta^{\prime}}^{\alpha \beta}\left(x, x^{\prime}\right) \theta_{ \pm}(-\sigma-\epsilon)
$$

as $\epsilon \rightarrow 0^{+}$. When we substitute this into the left-hand side of Equation (351) and then take the limit, we obtain

$$
\begin{aligned}
\square G_{ \pm \gamma^{\prime} \delta^{\prime}}^{\alpha \beta}+2 R_{\gamma \delta}^{\alpha \beta} G_{ \pm \gamma^{\prime} \delta^{\prime}}^{\gamma \delta}= & -4 \pi \delta_{4}\left(x, x^{\prime}\right) U_{\gamma^{\prime} \delta^{\prime}}^{\alpha \beta}+\delta_{ \pm}^{\prime}(\sigma)\left\{2 U_{\gamma^{\prime} \delta^{\prime} ; \gamma^{\prime}}^{\alpha \beta}+\left(\sigma_{\gamma}^{\gamma}-4\right) U_{\gamma^{\prime} \delta^{\prime}}^{\alpha \beta}\right\} \\
& +\delta_{ \pm}(\sigma)\left\{-2 V_{\gamma^{\prime} \delta^{\prime} ; \gamma^{\alpha}}^{\alpha \beta} \sigma^{\gamma}+\left(2-\sigma_{\gamma}^{\gamma}\right) V_{\gamma^{\prime} \delta^{\prime}}^{\alpha \beta}+\square U_{\gamma^{\prime} \delta^{\prime}}^{\alpha \beta}+2 R_{\gamma^{\prime} \delta}^{\alpha \beta} U_{\gamma^{\prime} \delta^{\prime}}^{\gamma \delta}\right\} \\
& +\theta_{ \pm}(-\sigma)\left\{\square V_{\gamma^{\prime} \delta^{\prime}}^{\alpha \beta}+2 R_{\gamma \delta}^{\alpha \beta} V_{\gamma^{\prime} \delta^{\prime}}^{\gamma \delta}\right\}
\end{aligned}
$$

after a routine computation similar to the one presented at the beginning of Section 4.3.2. Comparison with Equation (351) returns

- the equations

$$
\left[\begin{array}{l}
U_{\gamma^{\prime} \delta^{\prime}}^{\alpha \beta} \\
g_{\gamma^{\prime}}
\end{array} g_{\delta^{\prime}}^{\beta)}\right]=\delta_{\gamma^{\prime}}^{\left(\alpha^{\prime}\right.} \delta_{\delta^{\prime}}^{\left.\beta^{\prime}\right)}
$$

and

$$
2 U_{\gamma^{\prime} \delta^{\prime} ; \gamma}^{\alpha \beta} \sigma^{\gamma}+\left(\sigma_{\gamma}^{\gamma}-4\right) U_{\gamma^{\prime} \delta^{\prime}}^{\alpha \beta}=0
$$

that determine $U_{\gamma^{\prime} \delta^{\prime}}^{\alpha \beta}\left(x, x^{\prime}\right)$; 
- the equation

$$
\check{V}_{\gamma^{\prime} \delta^{\prime} ; \gamma}^{\alpha \beta} \sigma^{\gamma}+\frac{1}{2}\left(\sigma_{\gamma}^{\gamma}-2\right) \check{V}_{\gamma^{\prime} \delta^{\prime}}^{\alpha \beta}=\left.\frac{1}{2}\left(\square U_{\gamma^{\prime} \delta^{\prime}}^{\alpha \beta}+2 R_{\gamma \delta}^{\alpha \beta} U_{\gamma^{\prime} \delta^{\prime}}^{\gamma \delta}\right)\right|_{\sigma=0}
$$

that determines $\check{V}_{\gamma^{\prime} \delta^{\prime}}^{\alpha \beta}\left(x, x^{\prime}\right)$, the restriction of $V_{\gamma^{\prime} \delta^{\prime}}^{\alpha \beta}\left(x, x^{\prime}\right)$ on the light cone $\sigma\left(x, x^{\prime}\right)=0$; and

- the wave equation

$$
\square V_{\gamma^{\prime} \delta^{\prime}}^{\alpha \beta}+2 R_{\gamma \delta}^{\alpha \beta} V_{\gamma^{\prime} \delta^{\prime}}^{\gamma \delta}=0
$$

that determines $V_{\gamma^{\prime} \delta^{\prime}}^{\alpha \beta}\left(x, x^{\prime}\right)$ inside the light cone.

Equation (354) can be integrated along the unique geodesic $\beta$ that links $x^{\prime}$ to $x$. The initial conditions are provided by Equation (353), and if we set $U_{\gamma^{\prime} \delta^{\prime}}^{\alpha \beta}\left(x, x^{\prime}\right)=g_{\gamma^{\prime}}^{(\alpha} g_{\delta^{\prime}}^{\beta)} U\left(x, x^{\prime}\right)$, we find that these equations reduce to Equations (272) and (271), respectively. According to Equation (273), then, we have

$$
U_{\gamma^{\prime} \delta^{\prime}}^{\alpha \beta}\left(x, x^{\prime}\right)=g_{\gamma^{\prime}}^{(\alpha}\left(x, x^{\prime}\right) g_{\delta^{\prime}}^{\beta)}\left(x, x^{\prime}\right) \Delta^{1 / 2}\left(x, x^{\prime}\right)
$$

which reduces to

$$
U_{\gamma^{\prime} \delta^{\prime}}^{\alpha \beta}=g_{\gamma^{\prime}}^{(\alpha} g_{\delta^{\prime}}^{\beta)}\left(1+\mathcal{O}\left(\lambda^{3}\right)\right)
$$

near coincidence, with $\lambda$ denoting the affine-parameter distance between $x^{\prime}$ and $x$; there is no term of order $\lambda^{2}$ because, by assumption, the background Ricci tensor vanishes at $x^{\prime}$ (as it does in the entire spacetime). Differentiation of this relation gives

$$
\begin{aligned}
U_{\gamma^{\prime} \delta^{\prime} ; \epsilon}^{\alpha \beta} & =\frac{1}{2} g_{\alpha^{\prime}}^{(\alpha} g_{\beta^{\prime}}^{\beta)} g_{\epsilon}^{\epsilon_{\epsilon}^{\prime}}\left(R_{\gamma^{\prime} \epsilon^{\prime} \iota^{\prime}}^{\alpha^{\prime}} \delta_{\delta^{\prime}}^{\beta^{\prime}}+R_{\delta^{\prime} \epsilon^{\prime} \iota^{\prime}}^{\alpha^{\prime}} \delta_{\gamma^{\prime}}^{\beta^{\prime}}\right) \sigma^{\iota^{\prime}}+\mathcal{O}\left(\lambda^{2}\right), \\
U_{\gamma^{\prime} \delta^{\prime} ; \epsilon^{\prime}}^{\alpha \beta} & =\frac{1}{2} g_{\alpha^{\prime}}^{(\alpha} g_{\beta^{\prime}}^{\beta)}\left(R_{\gamma^{\prime} \epsilon^{\prime} \iota^{\prime}}^{\alpha^{\prime}} \delta_{\delta^{\prime}}^{\beta^{\prime}}+R_{\delta^{\prime} \epsilon^{\prime} \iota^{\prime}}^{\alpha^{\prime}}{ }_{\gamma^{\prime}}^{\beta^{\prime}}\right) \sigma^{\iota^{\prime}}+\mathcal{O}\left(\lambda^{2}\right),
\end{aligned}
$$

and eventually,

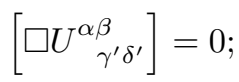

this last result follows from the fact that $\left[U_{\gamma^{\prime} \delta^{\prime} ; \epsilon \iota}^{\alpha \beta}\right]$ is antisymmetric in the last pair of indices.

Similarly, Equation (355) can be integrated along each null geodesic that generates the null cone $\sigma\left(x, x^{\prime}\right)=0$. The initial values are obtained by taking the coincidence limit of this equation, using Equations (353), (361), and the additional relation $\left[\sigma_{\gamma}^{\gamma}\right]=4$. We arrive at

$$
\left[\begin{array}{c}
\alpha \beta \\
\gamma^{\prime} \delta^{\prime}
\end{array}\right]=\frac{1}{2}\left(R_{\gamma^{\prime} \delta^{\prime}}^{\alpha^{\prime} \beta^{\prime}}+R_{\gamma^{\prime} \delta^{\prime}}^{\beta^{\prime} \alpha^{\prime}}\right)
$$

With the characteristic data obtained by integrating Equation (355), the wave equation of Equation (356) admits a unique solution.

To summarize, the retarded and advanced gravitational Green's functions are given by Equation (352) with $U_{\gamma^{\prime} \delta^{\prime}}^{\alpha \beta}\left(x, x^{\prime}\right)$ given by Equation (357) and $V_{\gamma^{\prime} \delta^{\prime}}^{\alpha \beta}\left(x, x^{\prime}\right)$ determined by Equation (356), and the characteristic data constructed with Equations (355) and (362). It should be emphasized that the construction provided in this section is restricted to $\mathcal{N}\left(x^{\prime}\right)$, the normal convex neighbourhood of the reference point $x^{\prime}$.

Living Reviews in Relativity

http://www. livingreviews . org/lrr-2004-6 


\subsubsection{Reciprocity and Kirchhoff representation}

The (globally defined) gravitational Green's functions satisfy the reciprocity relation

$$
G_{\gamma^{\prime} \delta^{\prime} \alpha \beta}^{-}\left(x^{\prime}, x\right)=G_{\alpha \beta \gamma^{\prime} \delta^{\prime}}^{+}\left(x, x^{\prime}\right) .
$$

The derivation of this result is virtually identical to what was presented in Sections 4.3.3 and 4.4.3. A direct consequence of the reciprocity relation is the statement

$$
V_{\gamma^{\prime} \delta^{\prime} \alpha \beta}\left(x^{\prime}, x\right)=V_{\alpha \beta \gamma^{\prime} \delta^{\prime}}\left(x, x^{\prime}\right) .
$$

The Kirchhoff representation for the trace-reversed gravitational perturbation $\gamma_{\alpha \beta}$ is formulated as follows. Suppose that $\gamma^{\alpha \beta}(x)$ satisfies the homogeneous version of Equation (349) and that initial values $\gamma^{\alpha^{\prime} \beta^{\prime}}\left(x^{\prime}\right), n^{\gamma^{\prime}} \nabla_{\gamma^{\prime}} \gamma^{\alpha^{\prime} \beta^{\prime}}\left(x^{\prime}\right)$ are specified on a spacelike hypersurface $\Sigma$. Then the value of the perturbation field at a point $x$ in the future of $\Sigma$ is given by

$$
\gamma^{\alpha \beta}(x)=-\frac{1}{4 \pi} \int_{\Sigma}\left(G_{+}^{\alpha \beta} \gamma^{\prime} \delta^{\prime}\left(x, x^{\prime}\right) \nabla^{\epsilon^{\prime}} \gamma^{\gamma^{\prime} \delta^{\prime}}\left(x^{\prime}\right)-\gamma^{\gamma^{\prime} \delta^{\prime}}\left(x^{\prime}\right) \nabla^{\epsilon^{\prime}} G_{+\gamma^{\prime} \delta^{\prime}}^{\alpha \beta}\left(x, x^{\prime}\right)\right) d \Sigma_{\epsilon^{\prime}},
$$

where $d \Sigma_{\epsilon^{\prime}}=-n_{\epsilon^{\prime}} d V$ is a surface element on $\Sigma ; n_{\epsilon^{\prime}}$ is the future-directed unit normal and $d V$ is the invariant volume element on the hypersurface. The derivation of Equation (365) is virtually identical to what was presented in Sections 4.3.4 and 4.4.3.

\subsubsection{Singular and radiative Green's functions}

We shall now construct singular and radiative Green's functions for the linearized gravitational field. The treatment here parallels closely what was presented in Sections 4.3.5 and 4.4.4.

We begin by introducing the bitensor $H_{\gamma^{\prime} \delta^{\prime}}^{\alpha \beta}\left(x, x^{\prime}\right)$ with properties

Gr.H1: $H_{\gamma^{\prime} \delta^{\prime}}^{\alpha \beta}\left(x, x^{\prime}\right)$ satisfies the homogeneous wave equation,

$$
\square H_{\gamma^{\prime} \delta^{\prime}}^{\alpha \beta}\left(x, x^{\prime}\right)+2 R_{\gamma^{\prime} \delta}^{\alpha \beta}(x) H_{\gamma^{\prime} \delta^{\prime}}^{\gamma \delta}\left(x, x^{\prime}\right)=0 ;
$$

Gr.H2: $H_{\gamma^{\prime} \delta^{\prime}}^{\alpha \beta}\left(x, x^{\prime}\right)$ is symmetric in its indices and arguments,

$$
H_{\gamma^{\prime} \delta^{\prime} \alpha \beta}\left(x^{\prime}, x\right)=H_{\alpha \beta \gamma^{\prime} \delta^{\prime}}\left(x, x^{\prime}\right) ;
$$
$\begin{aligned} \text { Gr.H3: } H^{\alpha \beta} \gamma^{\prime} \delta^{\prime} & \left(x, x^{\prime}\right) \text { agrees with the retarded Green's function if } x \text { is in the chronological future } \\ & \text { of } x^{\prime} \text {, }\end{aligned}$

$$
H_{\gamma^{\prime} \delta^{\prime}}^{\alpha \beta}\left(x, x^{\prime}\right)=G_{+\gamma^{\prime} \delta^{\prime}}^{\alpha \beta}\left(x, x^{\prime}\right) \quad \text { when } x \in I^{+}\left(x^{\prime}\right) ;
$$
$\begin{aligned} \text { Gr.H4: } & H^{\alpha \beta} \gamma^{\prime} \delta^{\prime} \\ & \text { of } x^{\prime},\end{aligned}$

$$
H_{\gamma^{\prime} \delta^{\prime}}^{\alpha \beta}\left(x, x^{\prime}\right)=G_{-\gamma^{\prime} \delta^{\prime}}^{\alpha \beta}\left(x, x^{\prime}\right) \quad \text { when } x \in I^{-}\left(x^{\prime}\right) .
$$

It is easy to prove that Property Gr.H4 follows from Property Gr.H2, Property Gr.H3, and the reciprocity relation (363) satisfied by the retarded and advanced Green's functions. That such a bitensor exists can be argued along the same lines as those presented in Section 4.3.5.

Equipped with $H_{\gamma^{\prime} \delta^{\prime}}^{\alpha \beta}\left(x, x^{\prime}\right)$ we define the singular Green's function to be

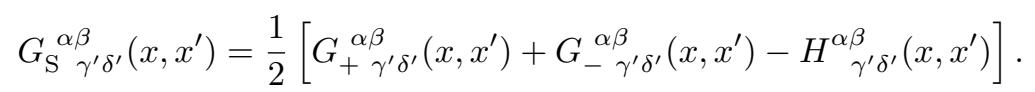

This comes with the properties 


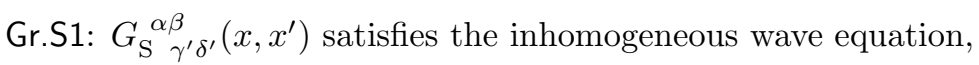

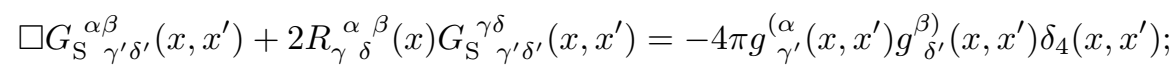

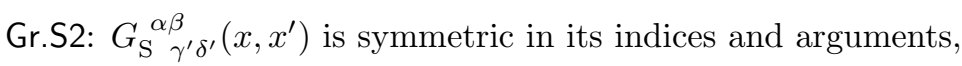

$$
G_{\gamma^{\prime} \delta^{\prime} \alpha \beta}^{\mathrm{S}}\left(x^{\prime}, x\right)=G_{\alpha \beta \gamma^{\prime} \delta^{\prime}}^{\mathrm{S}}\left(x, x^{\prime}\right)
$$

Gr.S3: $G_{\mathrm{S}}^{\alpha \beta} \gamma^{\prime} \delta^{\prime}\left(x, x^{\prime}\right)$ vanishes if $x$ is in the chronological past or future of $x^{\prime}$,

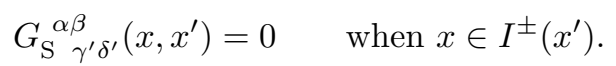

These can be established as consequences of Properties Gr.H1, Gr.H2, Gr.H3, and Gr.H4, and the properties of the retarded and advanced Green's functions.

The radiative Green's function is then defined by

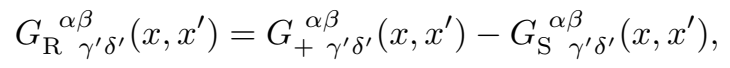

and it comes with the properties

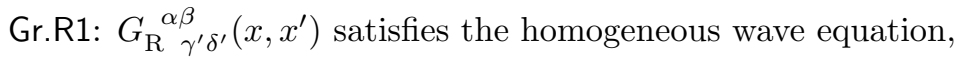

$$
\square G_{\mathrm{R}}^{\alpha \beta \gamma^{\prime} \delta^{\prime}}\left(x, x^{\prime}\right)+2 R_{\gamma \delta}^{\alpha \beta}(x) G_{\mathrm{R}}^{\gamma \delta \gamma^{\prime} \delta^{\prime}}\left(x, x^{\prime}\right)=0 ;
$$

Gr.R2: $G_{\mathrm{R}}^{\alpha \beta} \gamma^{\prime} \delta^{\prime}\left(x, x^{\prime}\right)$ agrees with the retarded Green's function if $x$ is in the chronological future of $x^{\prime}$,

$$
G_{\mathrm{R}}^{\alpha \beta} \gamma^{\prime} \delta^{\prime}\left(x, x^{\prime}\right)=G_{+\gamma^{\prime} \delta^{\prime}}^{\alpha \beta}\left(x, x^{\prime}\right) \quad \text { when } x \in I^{+}\left(x^{\prime}\right) ;
$$

Gr.R3: $G_{\mathrm{R}}^{\alpha \beta} \gamma^{\prime} \delta^{\prime}\left(x, x^{\prime}\right)$ vanishes if $x$ is in the chronological past of $x^{\prime}$,

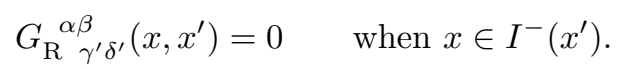

Those follow immediately from Properties Gr.S1, Gr.S2, and Gr.S3, and the properties of the retarded Green's function.

When $x$ is restricted to the normal convex neighbourhood of $x^{\prime}$, we have the explicit relations

$$
\begin{aligned}
H_{\gamma^{\prime} \delta^{\prime}}^{\alpha \beta}\left(x, x^{\prime}\right) & =V_{\gamma^{\prime} \delta^{\prime}}^{\alpha \beta}\left(x, x^{\prime}\right), \\
G_{\mathrm{S}}^{\alpha \beta} \gamma^{\prime} \delta^{\prime} & \left(x, x^{\prime}\right)=\frac{1}{2} U_{\gamma^{\prime} \delta^{\prime}}^{\alpha \beta}\left(x, x^{\prime}\right) \delta(\sigma)-\frac{1}{2} V_{\gamma^{\prime} \delta^{\prime}}^{\alpha \beta}\left(x, x^{\prime}\right) \theta(\sigma), \\
G_{\mathrm{R}}^{\alpha \beta} \gamma^{\prime} \delta^{\prime} & \left(x, x^{\prime}\right)=\frac{1}{2} U_{\gamma^{\prime} \delta^{\prime}}^{\alpha \beta}\left(x, x^{\prime}\right)\left[\delta_{+}(\sigma)-\delta_{-}(\sigma)\right]+V_{\gamma^{\prime} \delta^{\prime}}^{\alpha \beta}\left(x, x^{\prime}\right)\left[\theta_{+}(-\sigma)+\frac{1}{2} \theta(\sigma)\right] .
\end{aligned}
$$

From these we see clearly that the singular Green's function does not distinguish between past and future (Property Gr.S2), and that its support excludes $I^{ \pm}\left(x^{\prime}\right)$ (Property Gr.S3). We see also that the radiative Green's function coincides with $G_{+\gamma^{\prime} \delta^{\prime}}^{\alpha \beta}\left(x, x^{\prime}\right)$ in $I^{+}\left(x^{\prime}\right)$ (Property Gr.R2), and that its support does not include $I^{-}\left(x^{\prime}\right)$ (Property Gr.R3).

Living Reviews in Relativity

http: //www . livingreviews . org//rr-2004-6 


\section{Motion of Point Particles}

\subsection{Motion of a scalar charge}

\subsubsection{Dynamics of a point scalar charge}

A point particle carries a scalar charge $q$ and moves on a world line $\gamma$ described by relations $z^{\mu}(\lambda)$, in which $\lambda$ is an arbitrary parameter. The particle generates a scalar potential $\Phi(x)$ and a field $\Phi_{\alpha}(x) \equiv \nabla_{\alpha} \Phi(x)$. The dynamics of the entire system is governed by the action

$$
S=S_{\text {field }}+S_{\text {particle }}+S_{\text {interaction }}
$$

where $S_{\text {field }}$ is an action functional for a free scalar field in a spacetime with metric $g_{\alpha \beta}, S_{\text {particle }}$ is the action of a free particle moving on a world line $\gamma$ in this spacetime, and $S_{\text {interaction }}$ is an interaction term that couples the field to the particle.

The field action is given by

$$
S_{\text {field }}=-\frac{1}{8 \pi} \int\left(g^{\alpha \beta} \Phi_{\alpha} \Phi_{\beta}+\xi R \Phi^{2}\right) \sqrt{-g} d^{4} x,
$$

where the integration is over all of spacetime; the field is coupled to the Ricci scalar $R$ by an arbitrary constant $\xi$. The particle action is

$$
S_{\text {particle }}=-m_{0} \int_{\gamma} d \tau
$$

where $m_{0}$ is the bare mass of the particle and $d \tau=\sqrt{-g_{\mu \nu}(z) \dot{z}^{\mu} \dot{z}^{\nu}} d \lambda$ is the differential of proper time along the world line; we use an overdot on $z^{\mu}(\lambda)$ to indicate differentiation with respect to the parameter $\lambda$. Finally, the interaction term is given by

$$
S_{\text {interaction }}=q \int_{\gamma} \Phi(z) d \tau=q \int \Phi(x) \delta_{4}(x, z) \sqrt{-g} d^{4} x d \tau .
$$

Notice that both $S_{\text {particle }}$ and $S_{\text {interaction }}$ are invariant under a reparameterization $\lambda \rightarrow \lambda^{\prime}(\lambda)$ of the world line.

Demanding that the total action be stationary under a variation $\delta \Phi(x)$ of the field configuration yields the wave equation

$$
(\square-\xi R) \Phi(x)=-4 \pi \mu(x)
$$

for the scalar potential, with a charge density $\mu(x)$ defined by

$$
\mu(x)=q \int_{\gamma} \delta_{4}(x, z) d \tau .
$$

These equations determine the field $\Phi_{\alpha}(x)$ once the motion of the scalar charge is specified. On the other hand, demanding that the total action be stationary under a variation $\delta z^{\mu}(\lambda)$ of the world line yields the equations of motion for the scalar charge,

$$
m(\tau) \frac{D u^{\mu}}{d \tau}=q\left(g^{\mu \nu}+u^{\mu} u^{\nu}\right) \Phi_{\nu}(z) .
$$

We have here adopted $\tau$ as the parameter on the world line, and introduced the four-velocity $u^{\mu}(\tau) \equiv d z^{\mu} / d \tau$. The dynamical mass that appears in Equation (387) is defined by $m(\tau)=$ $m_{0}-q \Phi(z)$, which can also be written in differential form as

$$
\frac{d m}{d \tau}=-q \Phi_{\mu}(z) u^{\mu}
$$


It should be clear that Equations (387) and (388) are valid only in a formal sense, because the scalar potential obtained from Equations (385) and (386) diverges on the world line. Before we can make sense of these equations we have to analyze the field's singularity structure near the world line.

\subsubsection{Retarded potential near the world line}

The retarded solution to Equation $(385)$ is $\Phi(x)=\int G_{+}\left(x, x^{\prime}\right) \mu\left(x^{\prime}\right) \sqrt{g^{\prime}} d^{4} x^{\prime}$, where $G_{+}\left(x, x^{\prime}\right)$ is the retarded Green's function introduced in Section 4.3. After substitution of Equation (386) we obtain

$$
\Phi(x)=q \int_{\gamma} G_{+}(x, z) d \tau,
$$

in which $z(\tau)$ gives the description of the world line $\gamma$. Because the retarded Green's function is defined globally in the entire spacetime, Equation (389) applies to any field point $x$.

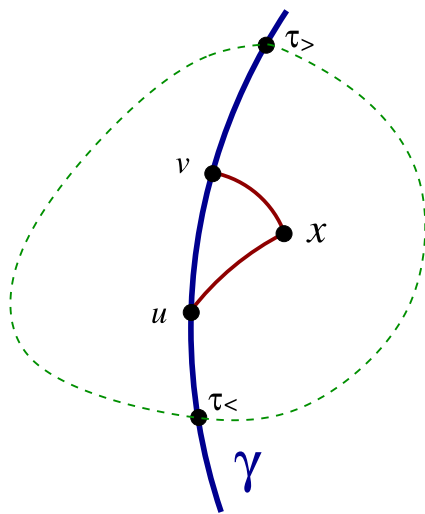

Figure 9: The region within the dashed boundary represents the normal convex neighbourhood of the point $x$. The world line $\gamma$ enters the neighbourhood at proper time $\tau_{<}$and exits at proper time $\tau_{>}$. Also shown are the retarded point $z(u)$ and the advanced point $z(v)$.

We now specialize Equation (389) to a point $x$ near the world line (see Figure 9). We let $\mathcal{N}(x)$ be the normal convex neighbourhood of this point, and we assume that the world line traverses $\mathcal{N}(x)$. Let $\tau_{<}$be the value of the proper-time parameter at which $\gamma$ enters $\mathcal{N}(x)$ from the past, and let $\tau_{>}$be its value when the world line leaves $\mathcal{N}(x)$. Then Equation (389) can be broken down into the three integrals

$$
\Phi(x)=q \int_{-\infty}^{\tau_{<}} G_{+}(x, z) d \tau+q \int_{\tau_{<}}^{\tau_{>}} G_{+}(x, z) d \tau+q \int_{\tau_{>}}^{\infty} G_{+}(x, z) d \tau .
$$

The third integration vanishes because $x$ is then in the past of $z(\tau)$, and $G_{+}(x, z)=0$. For the second integration, $x$ is the normal convex neighbourhood of $z(\tau)$, and the retarded Green's function can be expressed in the Hadamard form produced in Section 4.3.2. This gives

$$
\int_{\tau_{<}}^{\tau_{>}} G_{+}(x, z) d \tau=\int_{\tau_{<}}^{\tau_{>}} U(x, z) \delta_{+}(\sigma) d \tau+\int_{\tau_{<}}^{\tau_{>}} V(x, z) \theta_{+}(-\sigma) d \tau,
$$

and to evaluate this we refer back to Section 3.3 and let $x^{\prime} \equiv z(u)$ be the retarded point associated with $x$; these points are related by $\sigma\left(x, x^{\prime}\right)=0$ and $r \equiv \sigma_{\alpha^{\prime}} u^{\alpha^{\prime}}$ is the retarded distance between $x$ and the world line. We resume the index convention of Section 3.3: To tensors at $x$ we assign

Living Reviews in Relativity

http://www. livingreviews . org//rr-2004-6 
indices $\alpha, \beta$, etc.; to tensors at $x^{\prime}$ we assign indices $\alpha^{\prime}, \beta^{\prime}$, etc.; and to tensors at a generic point $z(\tau)$ on the world line we assign indices $\mu, \nu$, etc.

To perform the first integration we change variables from $\tau$ to $\sigma$, noticing that $\sigma$ increases as $z(\tau)$ passes through $x^{\prime}$. The change of $\sigma$ on the world line is given by $d \sigma \equiv \sigma(x, z+d z)-\sigma(x, z)=$ $\sigma_{\mu} u^{\mu} d \tau$, and we find that the first integral evaluates to $U(x, z) /\left(\sigma_{\mu} u^{\mu}\right)$ with $z$ identified with $x^{\prime}$. The second integration is cut off at $\tau=u$ by the step function, and we obtain our final expression for the retarded potential of a point scalar charge:

$$
\Phi(x)=\frac{q}{r} U\left(x, x^{\prime}\right)+q \int_{\tau_{<}}^{u} V(x, z) d \tau+q \int_{-\infty}^{\tau_{<}} G_{+}(x, z) d \tau .
$$

This expression applies to a point $x$ sufficiently close to the world line that there exists a nonempty intersection between $\mathcal{N}(x)$ and $\gamma$.

\subsubsection{Field of a scalar charge in retarded coordinates}

When we differentiate the potential of Equation (390) we must keep in mind that a variation in $x$ induces a variation in $x^{\prime}$ because the new points $x+\delta x$ and $x^{\prime}+\delta x^{\prime}$ must also be linked by a null geodesic - you may refer back to Section 3.3.2 for a detailed discussion. This means, for example, that the total variation of $U\left(x, x^{\prime}\right)$ is $\delta U=U\left(x+\delta x, x^{\prime}+\delta x^{\prime}\right)-U\left(x, x^{\prime}\right)=U_{; \alpha} \delta x^{\alpha}+U_{; \alpha^{\prime}} u^{\alpha^{\prime}} \delta u$. The gradient of the scalar potential is therefore given by

$$
\Phi_{\alpha}(x)=-\frac{q}{r^{2}} U\left(x, x^{\prime}\right) \partial_{\alpha} r+\frac{q}{r} U_{; \alpha}\left(x, x^{\prime}\right)+\frac{q}{r} U_{; \alpha^{\prime}}\left(x, x^{\prime}\right) u^{\alpha^{\prime}} \partial_{\alpha} u+q V\left(x, x^{\prime}\right) \partial_{\alpha} u+\Phi_{\alpha}^{\mathrm{tail}}(x),
$$

where the "tail integral" is defined by

$$
\begin{aligned}
\Phi_{\alpha}^{\mathrm{tail}}(x) & =q \int_{\tau_{<}}^{u} \nabla_{\alpha} V(x, z) d \tau+q \int_{-\infty}^{\tau_{<}} \nabla_{\alpha} G_{+}(x, z) d \tau \\
& =q \int_{-\infty}^{u^{-}} \nabla_{\alpha} G_{+}(x, z) d \tau .
\end{aligned}
$$

In the second form of the definition we integrate $\nabla_{\alpha} G_{+}(x, z)$ from $\tau=-\infty$ to almost $\tau=u$, but we cut the integration short at $\tau=u^{-} \equiv u-0^{+}$to avoid the singular behaviour of the retarded Green's function at $\sigma=0$. This limiting procedure gives rise to the first form of the definition, with the advantage that the integral need not be broken down into contributions that refer to $\mathcal{N}(x)$ and its complement, respectively.

We shall now expand $\Phi_{\alpha}(x)$ in powers of $r$, and express the results in terms of the retarded coordinates $\left(u, r, \Omega^{a}\right)$ introduced in Section 3.3. It will be convenient to decompose $\Phi_{\alpha}(x)$ in the tetrad $\left(e_{0}^{\alpha}, e_{a}^{\alpha}\right)$ that is obtained by parallel transport of $\left(u^{\alpha^{\prime}}, e_{a}^{\alpha^{\prime}}\right)$ on the null geodesic that links $x$ to $x^{\prime} \equiv z(u)$; this construction is detailed in Section 3.3. Note that throughout this section we set $\omega_{a b}=0$, where $\omega_{a b}$ is the rotation tensor defined by Equation (138): The tetrad vectors $e_{a}^{\alpha^{\prime}}$ are taken to be Fermi-Walker transported on $\gamma$. The expansion relies on Equation (166) for $\partial_{\alpha} u$, Equation (168) for $\partial_{\alpha} r$, and we shall need

$$
U\left(x, x^{\prime}\right)=1+\frac{1}{12} r^{2}\left(R_{00}+2 R_{0 a} \Omega^{a}+R_{a b} \Omega^{a} \Omega^{b}\right)+\mathcal{O}\left(r^{3}\right),
$$

which follows from Equation (275) and the relation $\sigma^{\alpha^{\prime}}=-r\left(u^{\alpha^{\prime}}+\Omega^{a} e_{a}^{\alpha^{\prime}}\right)$ first encountered in Equation (144); recall that

$$
R_{00}(u)=R_{\alpha^{\prime} \beta^{\prime}} u^{\alpha^{\prime}} u^{\beta^{\prime}}, \quad R_{0 a}(u)=R_{\alpha^{\prime} \beta^{\prime}} u^{\alpha^{\prime}} e_{a}^{\beta^{\prime}}, \quad R_{a b}(u)=R_{\alpha^{\prime} \beta^{\prime}} e_{a}^{\alpha^{\prime}} e_{b}^{\beta^{\prime}}
$$


are frame components of the Ricci tensor evaluated at $x^{\prime}$. We shall also need the expansions

$$
U_{; \alpha}\left(x, x^{\prime}\right)=\frac{1}{6} r g_{\alpha}^{\alpha^{\prime}}\left(R_{\alpha^{\prime} 0}+R_{\alpha^{\prime} b} \Omega^{b}\right)+\mathcal{O}\left(r^{2}\right)
$$

and

$$
U_{; \alpha^{\prime}}\left(x, x^{\prime}\right) u^{\alpha^{\prime}}=-\frac{1}{6} r\left(R_{00}+R_{0 a} \Omega^{a}\right)+\mathcal{O}\left(r^{2}\right),
$$

which follow from Equations (276); recall from Equation (141) that the parallel propagator can be expressed as $g_{\alpha}^{\alpha^{\prime}}=u^{\alpha^{\prime}} e_{\alpha}^{0}+e_{a}^{\alpha^{\prime}} e_{\alpha}^{a}$. And finally, we shall need

$$
V\left(x, x^{\prime}\right)=\frac{1}{12}(1-6 \xi) R+\mathcal{O}(r)
$$

a relation that was first established in Equation (278); here $R \equiv R(u)$ is the Ricci scalar evaluated at $x^{\prime}$.

Collecting all these results gives

$$
\begin{aligned}
\Phi_{0}\left(u, r, \Omega^{a}\right) \equiv & \Phi_{\alpha}(x) e_{0}^{\alpha}(x) \\
= & \frac{q}{r} a_{a} \Omega^{a}+\frac{1}{2} q R_{a 0 b 0} \Omega^{a} \Omega^{b}+\frac{1}{12}(1-6 \xi) q R+\Phi_{0}^{\text {tail }}+\mathcal{O}(r) \\
\Phi_{a}\left(u, r, \Omega^{a}\right) \equiv & \Phi_{\alpha}(x) e_{a}^{\alpha}(x) \\
= & -\frac{q}{r^{2}} \Omega_{a}-\frac{q}{r} a_{b} \Omega^{b} \Omega_{a}-\frac{1}{3} q R_{b 0 c 0} \Omega^{b} \Omega^{c} \Omega_{a}-\frac{1}{6} q\left(R_{a 0 b 0} \Omega^{b}-R_{a b 0 c} \Omega^{b} \Omega^{c}\right) \\
& +\frac{1}{12} q\left[R_{00}-R_{b c} \Omega^{b} \Omega^{c}-(1-6 \xi) R\right] \Omega_{a}+\frac{1}{6} q\left(R_{a 0}+R_{a b} \Omega^{b}\right)+\Phi_{a}^{\text {tail }}+\mathcal{O}(r),
\end{aligned}
$$

where $a_{a}=a_{\alpha^{\prime}} e_{a}^{\alpha^{\prime}}$ are the frame components of the acceleration vector,

$$
R_{a 0 b 0}(u)=R_{\alpha^{\prime} \gamma^{\prime} \beta^{\prime} \delta^{\prime}} e_{a}^{\alpha^{\prime}} u^{\gamma^{\prime}} e_{b}^{\beta^{\prime}} u^{\delta^{\prime}}, \quad R_{a b 0 c}(u)=R_{\alpha^{\prime} \gamma^{\prime} \beta^{\prime} \delta^{\prime}} e_{a}^{\alpha^{\prime}} e_{b}^{\gamma^{\prime}} u^{\beta^{\prime}} e_{c}^{\delta^{\prime}}
$$

are frame components of the Riemann tensor evaluated at $x^{\prime}$, and

$$
\Phi_{0}^{\text {tail }}(u)=\Phi_{\alpha^{\prime}}^{\text {tail }}\left(x^{\prime}\right) u^{\alpha^{\prime}}, \quad \Phi_{a}^{\text {tail }}(u)=\Phi_{\alpha^{\prime}}^{\text {tail }}\left(x^{\prime}\right) e_{a}^{\alpha^{\prime}}
$$

are the frame components of the tail integral evaluated at $x^{\prime}$. Equations (397) and (398) show clearly that $\Phi_{\alpha}(x)$ is singular on the world line: The field diverges as $r^{-2}$ when $r \rightarrow 0$, and many of the terms that stay bounded in the limit depend on $\Omega^{a}$ and therefore possess a directional ambiguity at $r=0$.

\subsubsection{Field of a scalar charge in Fermi normal coordinates}

The gradient of the scalar potential can also be expressed in the Fermi normal coordinates of Section 3.2. To effect this translation we make $\bar{x} \equiv z(t)$ the new reference point on the world line. We resume here the notation of Section 3.4 and assign indices $\bar{\alpha}, \bar{\beta}, \ldots$ to tensors at $\bar{x}$. The Fermi normal coordinates are denoted $\left(t, s, \omega^{a}\right)$, and we let $\left(\bar{e}_{0}^{\alpha}, \bar{e}_{a}^{\alpha}\right)$ be the tetrad at $x$ that is obtained by parallel transport of $\left(u^{\bar{\alpha}}, e_{a}^{\bar{\alpha}}\right)$ on the spacelike geodesic that links $x$ to $\bar{x}$.

Our first task is to decompose $\Phi_{\alpha}(x)$ in the tetrad $\left(\bar{e}_{0}^{\alpha}, \bar{e}_{a}^{\alpha}\right)$, thereby defining $\bar{\Phi}_{0} \equiv \Phi_{\alpha} \bar{e}_{0}^{\alpha}$ and $\bar{\Phi}_{a} \equiv \Phi_{\alpha} \bar{e}_{a}^{\alpha}$. For this purpose we use Equations $(224,225)$ and $(397,398)$ to obtain

$$
\begin{aligned}
\bar{\Phi}_{0} & =\left[1+\mathcal{O}\left(r^{2}\right)\right] \Phi_{0}+\left[r\left(1-a_{b} \Omega^{b}\right) a^{a}+\frac{1}{2} r^{2} \dot{a}^{a}+\frac{1}{2} r^{2} R_{0 b 0}^{a} \Omega^{b}+\mathcal{O}\left(r^{3}\right)\right] \Phi_{a} \\
& =-\frac{1}{2} q \dot{a}_{a} \Omega^{a}+\frac{1}{12}(1-6 \xi) q R+\bar{\Phi}_{0}^{\text {tail }}+\mathcal{O}(r)
\end{aligned}
$$

Living Reviews in Relativity

http: //www . livingreviews . org/lrr-2004-6 
and

$$
\begin{aligned}
\bar{\Phi}_{a}= & {\left[\delta^{b}{ }_{a}+\frac{1}{2} r^{2} a^{b} a_{a}-\frac{1}{2} r^{2} R_{a 0 c}^{b} \Omega^{c}+\mathcal{O}\left(r^{3}\right)\right] \Phi_{b}+\left[r a_{a}+\mathcal{O}\left(r^{2}\right)\right] \Phi_{0} } \\
= & -\frac{q}{r^{2}} \Omega_{a}-\frac{q}{r} a_{b} \Omega^{b} \Omega_{a}+\frac{1}{2} q a_{b} \Omega^{b} a_{a}-\frac{1}{3} q R_{b 0 c 0} \Omega^{b} \Omega^{c} \Omega_{a}-\frac{1}{6} q R_{a 0 b 0} \Omega^{b}-\frac{1}{3} q R_{a b 0 c} \Omega^{b} \Omega^{c} \\
& +\frac{1}{12} q\left[R_{00}-R_{b c} \Omega^{b} \Omega^{c}-(1-6 \xi) R\right] \Omega_{a}+\frac{1}{6} q\left(R_{a 0}+R_{a b} \Omega^{b}\right)+\bar{\Phi}_{a}^{\mathrm{tail}}+\mathcal{O}(r),
\end{aligned}
$$

where all frame components are still evaluated at $x^{\prime}$, except for $\bar{\Phi}_{0}^{\text {tail }}$ and $\bar{\Phi}_{a}^{\text {tail }}$ which are evaluated at $\bar{x}$.

We must still translate these results into the Fermi normal coordinates $\left(t, s, \omega^{a}\right)$. For this we involve Equations $(221,222,223)$, from which we deduce, for example,

$$
\begin{aligned}
\frac{1}{r^{2}} \Omega_{a}= & \frac{1}{s^{2}} \omega_{a}+\frac{1}{2 s} a_{a}-\frac{3}{2 s} a_{b} \omega^{b} \omega_{a}-\frac{3}{4} a_{b} \omega^{b} a_{a}+\frac{15}{8}\left(a_{b} \omega^{b}\right)^{2} \omega_{a}+\frac{3}{8} \dot{a}_{0} \omega_{a}-\frac{1}{3} \dot{a}_{a} \\
& +\dot{a}_{b} \omega^{b} \omega_{a}+\frac{1}{6} R_{a 0 b 0} \omega^{b}-\frac{1}{2} R_{b 0 c 0} \omega^{b} \omega^{c} \omega_{a}-\frac{1}{3} R_{a b 0 c} \omega^{b} \omega^{c}+\mathcal{O}(s)
\end{aligned}
$$

and

$$
\frac{1}{r} a_{b} \Omega^{b} \Omega_{a}=\frac{1}{s} a_{b} \omega^{b} \omega_{a}+\frac{1}{2} a_{b} \omega^{b} a_{a}-\frac{3}{2}\left(a_{b} \omega^{b}\right)^{2} \omega_{a}-\frac{1}{2} \dot{a}_{0} \omega_{a}-\dot{a}_{b} \omega^{b} \omega_{a}+\mathcal{O}(s),
$$

in which all frame components (on the right-hand side of these relations) are now evaluated at $\bar{x}$; to obtain the second relation we expressed $a_{a}(u)$ as $a_{a}(t)-s \dot{a}_{a}(t)+\mathcal{O}\left(s^{2}\right)$, since according to Equation (221), $u=t-s+\mathcal{O}\left(s^{2}\right)$.

Collecting these results yields

$$
\begin{aligned}
\bar{\Phi}_{0}\left(t, s, \omega^{a}\right) \equiv & \Phi_{\alpha}(x) \bar{e}_{0}^{\alpha}(x) \\
= & -\frac{1}{2} q \dot{a}_{a} \omega^{a}+\frac{1}{12}(1-6 \xi) q R+\bar{\Phi}_{0}^{\text {tail }}+\mathcal{O}(s) \\
\bar{\Phi}_{a}\left(t, s, \omega^{a}\right) \equiv & \Phi_{\alpha}(x) \bar{e}_{a}^{\alpha}(x) \\
= & -\frac{q}{s^{2}} \omega_{a}-\frac{q}{2 s}\left(a_{a}-a_{b} \omega^{b} \omega_{a}\right)+\frac{3}{4} q a_{b} \omega^{b} a_{a}-\frac{3}{8} q\left(a_{b} \omega^{b}\right)^{2} \omega_{a}+\frac{1}{8} q \dot{a}_{0} \omega_{a}+\frac{1}{3} q \dot{a}_{a} \\
& -\frac{1}{3} q R_{a 0 b 0} \omega^{b}+\frac{1}{6} q R_{b 0 c 0} \omega^{b} \omega^{c} \omega_{a}+\frac{1}{12} q\left[R_{00}-R_{b c} \omega^{b} \omega^{c}-(1-6 \xi) R\right] \omega_{a} \\
& +\frac{1}{6} q\left(R_{a 0}+R_{a b} \omega^{b}\right)+\bar{\Phi}_{a}^{\text {tail }}+\mathcal{O}(s) .
\end{aligned}
$$

In these expressions, $a_{a}(t)=a_{\bar{\alpha}} e_{a}^{\bar{\alpha}}$ are the frame components of the acceleration vector evaluated at $\bar{x}, \dot{a}_{0}(t)=\dot{a}_{\bar{\alpha}} u^{\bar{\alpha}}$ and $\dot{a}_{a}(t)=\dot{a}_{\bar{\alpha}} e_{a}^{\bar{\alpha}}$ are frame components of its covariant derivative, $R_{a 0 b 0}(t)=$ $R_{\bar{\alpha} \bar{\gamma} \bar{\beta} \bar{\delta}} e_{a}^{\bar{\alpha}} u^{\bar{\gamma}} e_{b}^{\bar{\beta}} u^{\bar{\delta}}$ are frame components of the Riemann tensor evaluated at $\bar{x}$,

$$
R_{00}(t)=R_{\bar{\alpha} \bar{\beta}} u^{\bar{\alpha}} u^{\bar{\beta}}, \quad R_{0 a}(t)=R_{\bar{\alpha} \bar{\beta}} u^{\bar{\alpha}} e_{a}^{\bar{\beta}}, \quad R_{a b}(t)=R_{\bar{\alpha} \bar{\beta}} e_{a}^{\bar{\alpha}} e_{b}^{\bar{\beta}}
$$

are frame components of the Ricci tensor, and $R(t)$ is the Ricci scalar evaluated at $\bar{x}$. Finally, we have that

$$
\bar{\Phi}_{0}^{\text {tail }}(t)=\Phi_{\bar{\alpha}}^{\text {tail }}(\bar{x}) u^{\bar{\alpha}}, \quad \bar{\Phi}_{a}^{\text {tail }}(t)=\Phi_{\bar{\alpha}}^{\text {tail }}(\bar{x}) e_{a}^{\bar{\alpha}}
$$

are the frame components of the tail integral - see Equation (392) - evaluated at $\bar{x} \equiv z(t)$.

We shall now compute the averages of $\bar{\Phi}_{0}$ and $\bar{\Phi}_{a}$ over $S(t, s)$, a two-surface of constant $t$ and $s$; these will represent the mean value of the field at a fixed proper distance away from the world line, as measured in a reference frame that is momentarily comoving with the particle. 
The two-surface is charted by angles $\theta^{A}(A=1,2)$ and it is described, in the Fermi normal coordinates, by the parametric relations $\hat{x}^{a}=s \omega^{a}\left(\theta^{A}\right)$; a canonical choice of parameterization is $\omega^{a}=(\sin \theta \cos \phi, \sin \theta \sin \phi, \cos \theta)$. Introducing the transformation matrices $\omega_{A}^{a} \equiv \partial \omega^{a} / \partial \theta^{A}$, we find from Equation (127) that the induced metric on $S(t, s)$ is given by

$$
d s^{2}=s^{2}\left[\omega_{A B}-\frac{1}{3} s^{2} R_{A B}+\mathcal{O}\left(s^{3}\right)\right] d \theta^{A} d \theta^{B},
$$

where $\omega_{A B} \equiv \delta_{a b} \omega_{A}^{a} \omega_{B}^{b}$ is the metric of the unit two-sphere, and where $R_{A B} \equiv R_{a c b d} \omega_{A}^{a} \omega^{c} \omega_{B}^{b} \omega^{d}$ depends on $t$ and the angles $\theta^{A}$. From this we infer that the element of surface area is given by

$$
d \mathcal{A}=s^{2}\left[1-\frac{1}{6} s^{2} R_{a c b}^{c}(t) \omega^{a} \omega^{b}+\mathcal{O}\left(s^{3}\right)\right] d \Omega,
$$

where $d \Omega=\sqrt{\operatorname{det}\left[\omega_{A B}\right]} d^{2} \theta$ is an element of solid angle - in the canonical parameterization, $d \Omega=\sin \theta d \theta d \phi$. Integration of Equation (404) produces the total surface area of $S(t, s)$, and $\mathcal{A}=4 \pi s^{2}\left[1-\frac{1}{18} s^{2} R_{a b}^{a b}+\mathcal{O}\left(s^{3}\right)\right]$.

The averaged fields are defined by

$$
\left\langle\bar{\Phi}_{0}\right\rangle(t, s)=\frac{1}{\mathcal{A}} \oint_{S(t, s)} \bar{\Phi}_{0}\left(t, s, \theta^{A}\right) d \mathcal{A}, \quad\left\langle\bar{\Phi}_{a}\right\rangle(t, s)=\frac{1}{\mathcal{A}} \oint_{S(t, s)} \bar{\Phi}_{a}\left(t, s, \theta^{A}\right) d \mathcal{A},
$$

where the quantities to be integrated are scalar functions of the Fermi normal coordinates. The results

$$
\frac{1}{4 \pi} \oint \omega^{a} d \Omega=0, \quad \frac{1}{4 \pi} \oint \omega^{a} \omega^{b} d \Omega=\frac{1}{3} \delta^{a b}, \quad \frac{1}{4 \pi} \oint \omega^{a} \omega^{b} \omega^{c} d \Omega=0,
$$

are easy to establish, and we obtain

$$
\begin{aligned}
& \left\langle\bar{\Phi}_{0}\right\rangle=\frac{1}{12}(1-6 \xi) q R+\bar{\Phi}_{0}^{\text {tail }}+\mathcal{O}(s), \\
& \left\langle\bar{\Phi}_{a}\right\rangle=-\frac{q}{3 s} a_{a}+\frac{1}{3} q \dot{a}_{a}+\frac{1}{6} q R_{a 0}+\bar{\Phi}_{a}^{\text {tail }}+\mathcal{O}(s) .
\end{aligned}
$$

The averaged field is still singular on the world line. Regardless, we shall take the formal limit $s \rightarrow 0$ of the expressions displayed in Equations (407) and (408). In the limit the tetrad $\left(\bar{e}_{0}^{\alpha}, \bar{e}_{a}^{\alpha}\right)$ reduces to $\left(u^{\bar{\alpha}}, e_{a}^{\bar{\alpha}}\right)$, and we can reconstruct the field at $\bar{x}$ by invoking the completeness relations $\delta_{\bar{\beta}}^{\bar{\alpha}}=-u^{\bar{\alpha}} u_{\bar{\beta}}+e_{a}^{\bar{\alpha}} e_{\bar{\beta}}^{a}$. We thus obtain

$$
\left\langle\Phi_{\bar{\alpha}}\right\rangle=\lim _{s \rightarrow 0}\left(-\frac{q}{3 s}\right) a_{\bar{\alpha}}-\frac{1}{12}(1-6 \xi) q R u_{\bar{\alpha}}+q\left(g_{\bar{\alpha} \bar{\beta}}+u_{\bar{\alpha}} u_{\bar{\beta}}\right)\left(\frac{1}{3} \dot{a}^{\bar{\beta}}+\frac{1}{6} R_{\bar{\gamma}}^{\bar{\beta}} u^{\bar{\gamma}}\right)+\Phi_{\bar{\alpha}}^{\text {tail }},
$$

where the tail integral can be copied from Equation (392),

$$
\Phi_{\bar{\alpha}}^{\text {tail }}(\bar{x})=q \int_{-\infty}^{t^{-}} \nabla_{\bar{\alpha}} G_{+}(\bar{x}, z) d \tau .
$$

The tensors appearing in Equation (409) all refer to $\bar{x} \equiv z(t)$, which now stands for an arbitrary point on the world line $\gamma$.

\subsubsection{Singular and radiative fields}

The singular potential

$$
\Phi^{\mathrm{S}}(x)=q \int_{\gamma} G_{\mathrm{S}}(x, z) d \tau
$$

Living Reviews in Relativity

http://www. livingreviews.org/lrr-2004-6 
is the (unphysical) solution to Equations (385) and (386) that is obtained by adopting the singular Green's function of Equation (295) instead of the retarded Green's function. As we shall see, the resulting singular field $\Phi_{\alpha}^{\mathrm{S}}(x)$ reproduces the singular behaviour of the retarded solution; the difference, $\Phi_{\alpha}^{\mathrm{R}}(x)=\Phi_{\alpha}(x)-\Phi_{\alpha}^{\mathrm{S}}(x)$, is smooth on the world line.

To evaluate the integral of Equation (411) we assume once more that $x$ is sufficiently close to $\gamma$ that the world line traverses $\mathcal{N}(x)$ (refer back to Figure 9). As before we let $\tau_{<}$and $\tau_{>}$be the values of the proper-time parameter at which $\gamma$ enters and leaves $\mathcal{N}(x)$, respectively. Then Equation (411) can be broken down into the three integrals,

$$
\Phi^{\mathrm{S}}(x)=q \int_{-\infty}^{\tau_{<}} G_{\mathrm{S}}(x, z) d \tau+q \int_{\tau_{<}}^{\tau_{>}} G_{\mathrm{S}}(x, z) d \tau+q \int_{\tau_{>}}^{\infty} G_{\mathrm{S}}(x, z) d \tau .
$$

The first integration vanishes because $x$ is then in the chronological future of $z(\tau)$, and $G_{\mathrm{S}}(x, z)=0$ by Equation (286). Similarly, the third integration vanishes because $x$ is then in the chronological past of $z(\tau)$. For the second integration, $x$ is the normal convex neighbourhood of $z(\tau)$, the singular Green's function can be expressed in the Hadamard form of Equation (297), and we have

$$
\int_{\tau_{<}}^{\tau_{>}} G_{\mathrm{S}}(x, z) d \tau=\frac{1}{2} \int_{\tau_{<}}^{\tau_{>}} U(x, z) \delta_{+}(\sigma) d \tau+\frac{1}{2} \int_{\tau_{<}}^{\tau_{>}} U(x, z) \delta_{-}(\sigma) d \tau-\frac{1}{2} \int_{\tau_{<}}^{\tau_{>}} V(x, z) \theta(\sigma) d \tau .
$$

To evaluate these we re-introduce the retarded point $x^{\prime} \equiv z(u)$ and let $x^{\prime \prime} \equiv z(v)$ be the advanced point associated with $x$; we recall from Section 3.4.4 that these points are related by $\sigma\left(x, x^{\prime \prime}\right)=0$ and that $r_{\mathrm{adv}} \equiv-\sigma_{\alpha^{\prime \prime}} u^{\alpha^{\prime \prime}}$ is the advanced distance between $x$ and the world line.

To perform the first integration we change variables from $\tau$ to $\sigma$, noticing that $\sigma$ increases as $z(\tau)$ passes through $x^{\prime}$; the integral evaluates to $U\left(x, x^{\prime}\right) / r$. We do the same for the second integration, but we notice now that $\sigma$ decreases as $z(\tau)$ passes through $x^{\prime \prime}$; the integral evaluates to $U\left(x, x^{\prime \prime}\right) / r_{\text {adv }}$. The third integration is restricted to the interval $u \leq \tau \leq v$ by the step function, and we obtain our final expression for the singular potential of a point scalar charge:

$$
\Phi^{\mathrm{S}}(x)=\frac{q}{2 r} U\left(x, x^{\prime}\right)+\frac{q}{2 r_{\mathrm{adv}}} U\left(x, x^{\prime \prime}\right)-\frac{1}{2} q \int_{u}^{v} V(x, z) d \tau .
$$

We observe that $\Phi^{\mathrm{S}}(x)$ depends on the state of motion of the scalar charge between the retarded time $u$ and the advanced time $v$; contrary to what was found in Section 5.1.2 for the retarded potential, there is no dependence on the particle's remote past.

We use the techniques of Section 5.1.3 to differentiate the potential of Equation (412). We find

$$
\begin{aligned}
\Phi_{\alpha}^{\mathrm{S}}(x)= & -\frac{q}{2 r^{2}} U\left(x, x^{\prime}\right) \partial_{\alpha} r-\frac{q}{2 r_{\mathrm{adv}}{ }^{2}} U\left(x, x^{\prime \prime}\right) \partial_{\alpha} r_{\mathrm{adv}}+\frac{q}{2 r} U_{; \alpha}\left(x, x^{\prime}\right)+\frac{q}{2 r} U_{; \alpha^{\prime}}\left(x, x^{\prime}\right) u^{\alpha^{\prime}} \partial_{\alpha} u \\
& +\frac{q}{2 r_{\mathrm{adv}}} U_{; \alpha}\left(x, x^{\prime \prime}\right)+\frac{q}{2 r_{\mathrm{adv}}} U_{; \alpha^{\prime \prime}}\left(x, x^{\prime \prime}\right) u^{\alpha^{\prime \prime}} \partial_{\alpha} v+\frac{1}{2} q V\left(x, x^{\prime}\right) \partial_{\alpha} u-\frac{1}{2} q V\left(x, x^{\prime \prime}\right) \partial_{\alpha} v \\
& -\frac{1}{2} q \int_{u}^{v} \nabla_{\alpha} V(x, z) d \tau,
\end{aligned}
$$

and we would like to express this as an expansion in powers of $r$. For this we shall rely on results already established in Section 5.1.3, as well as additional expansions that will involve the advanced point $x^{\prime \prime}$. Those we develop now.

We recall first that a relation between retarded and advanced times was worked out in Equation (229), that an expression for the advanced distance was displayed in Equation (230), and that Equations (231) and (232) give expansions for $\partial_{\alpha} v$ and $\partial_{\alpha} r_{\text {adv }}$, respectively.

To derive an expansion for $U\left(x, x^{\prime \prime}\right)$ we follow the general method of Section 3.4.4 and define a function $U(\tau) \equiv U(x, z(\tau))$ of the proper-time parameter on $\gamma$. We have that

$$
U\left(x, x^{\prime \prime}\right) \equiv U(v)=U\left(u+\Delta^{\prime}\right)=U(u)+\dot{U}(u) \Delta^{\prime}+\frac{1}{2} \ddot{U}(u) \Delta^{\prime 2}+O\left(\Delta^{\prime 3}\right),
$$


where overdots indicate differentiation with respect to $\tau$, and where $\Delta^{\prime} \equiv v-u$. The leading term $U(u) \equiv U\left(x, x^{\prime}\right)$ was worked out in Equation (393), and the derivatives of $U(\tau)$ are given by

$$
\dot{U}(u)=U_{; \alpha^{\prime}} u^{\alpha^{\prime}}=-\frac{1}{6} r\left(R_{00}+R_{0 a} \Omega^{a}\right)+\mathcal{O}\left(r^{2}\right)
$$

and

$$
\ddot{U}(u)=U_{; \alpha^{\prime} \beta^{\prime}} u^{\alpha^{\prime}} u^{\beta^{\prime}}+U_{; \alpha^{\prime}} a^{\alpha^{\prime}}=\frac{1}{6} R_{00}+\mathcal{O}(r),
$$

according to Equations (395) and (276). Combining these results together with Equation (229) for $\Delta^{\prime}$ gives

$$
U\left(x, x^{\prime \prime}\right)=1+\frac{1}{12} r^{2}\left(R_{00}-2 R_{0 a} \Omega^{a}+R_{a b} \Omega^{a} \Omega^{b}\right)+\mathcal{O}\left(r^{3}\right),
$$

which should be compared with Equation (393). It should be emphasized that in Equation (414) and all equations below, the frame components of the Ricci tensor are evaluated at the retarded point $x^{\prime} \equiv z(u)$, and not at the advanced point. The preceding computation gives us also an expansion for $U_{; \alpha^{\prime \prime}} u^{\alpha^{\prime \prime}} \equiv \dot{U}(v)=\dot{U}(u)+\ddot{U}(u) \Delta^{\prime}+\mathcal{O}\left(\Delta^{\prime 2}\right)$. This becomes

$$
U_{; \alpha^{\prime \prime}}\left(x, x^{\prime \prime}\right) u^{\alpha^{\prime \prime}}=\frac{1}{6} r\left(R_{00}-R_{0 a} \Omega^{a}\right)+\mathcal{O}\left(r^{2}\right),
$$

which should be compared with Equation (395).

We proceed similarly to derive an expansion for $U_{; \alpha}\left(x, x^{\prime \prime}\right)$. Here we introduce the functions $U_{\alpha}(\tau) \equiv U_{; \alpha}(x, z(\tau))$ and express $U_{; \alpha}\left(x, x^{\prime \prime}\right)$ as $U_{\alpha}(v)=U_{\alpha}(u)+\dot{U}_{\alpha}(u) \Delta^{\prime}+\mathcal{O}\left(\Delta^{\prime 2}\right)$. The leading term $U_{\alpha}(u) \equiv U_{; \alpha}\left(x, x^{\prime}\right)$ was computed in Equation (394), and

$$
\dot{U}_{\alpha}(u)=U_{; \alpha \beta^{\prime}} u^{\beta^{\prime}}=-\frac{1}{6} g_{\alpha}^{\alpha^{\prime}} R_{\alpha^{\prime} 0}+\mathcal{O}(r)
$$

follows from Equation (276). Combining these results together with Equation (229) for $\Delta^{\prime}$ gives

$$
U_{; \alpha}\left(x, x^{\prime \prime}\right)=-\frac{1}{6} r g_{\alpha}^{\alpha^{\prime}}\left(R_{\alpha^{\prime} 0}-R_{\alpha^{\prime} b} \Omega^{b}\right)+\mathcal{O}\left(r^{2}\right),
$$

and this should be compared with Equation (394).

The last expansion we shall need is

$$
V\left(x, x^{\prime \prime}\right)=\frac{1}{12}(1-6 \xi) R+\mathcal{O}(r),
$$

which follows at once from Equation (396) and the fact that $V\left(x, x^{\prime \prime}\right)-V\left(x, x^{\prime}\right)=\mathcal{O}(r)$; the Ricci scalar is evaluated at the retarded point $x^{\prime}$.

It is now a straightforward (but tedious) matter to substitute these expansions (all of them!) into Equation (413) and obtain the projections of the singular field $\Phi_{\alpha}^{\mathrm{S}}(x)$ in the same tetrad $\left(e_{0}^{\alpha}, e_{a}^{\alpha}\right)$ that was employed in Section 5.1.3. This gives

$$
\begin{aligned}
\Phi_{0}^{\mathrm{S}}\left(u, r, \Omega^{a}\right) \equiv & \Phi_{\alpha}^{\mathrm{S}}(x) e_{0}^{\alpha}(x) \\
= & \frac{q}{r} a_{a} \Omega^{a}+\frac{1}{2} q R_{a 0 b 0} \Omega^{a} \Omega^{b}+\mathcal{O}(r) \\
\Phi_{a}^{\mathrm{S}}\left(u, r, \Omega^{a}\right) \equiv & \Phi_{\alpha}^{\mathrm{S}}(x) e_{a}^{\alpha}(x) \\
= & -\frac{q}{r^{2}} \Omega_{a}-\frac{q}{r} a_{b} \Omega^{b} \Omega_{a}-\frac{1}{3} q \dot{a}_{a}-\frac{1}{3} q R_{b 0 c 0} \Omega^{b} \Omega^{c} \Omega_{a}-\frac{1}{6} q\left(R_{a 0 b 0} \Omega^{b}-R_{a b 0 c} \Omega^{b} \Omega^{c}\right) \\
& +\frac{1}{12} q\left[R_{00}-R_{b c} \Omega^{b} \Omega^{c}-(1-6 \xi) R\right] \Omega_{a}+\frac{1}{6} q R_{a b} \Omega^{b},
\end{aligned}
$$

Living Reviews in Relativity

http://www. livingreviews.org/lrr-2004-6 
in which all frame components are evaluated at the retarded point $x^{\prime} \equiv z(u)$. Comparison of these expressions with Equations (397) and (398) reveals that the retarded and singular fields share the same singularity structure.

The difference between the retarded field of Equations $(397,398)$ and the singular field of Equations $(418,419)$ defines the radiative field $\Phi_{\alpha}^{\mathrm{R}}(x)$. Its tetrad components are

$$
\begin{aligned}
\Phi_{0}^{\mathrm{R}} & =\frac{1}{12}(1-6 \xi) q R+\Phi_{0}^{\mathrm{tail}}+\mathcal{O}(r), \\
\Phi_{a}^{\mathrm{R}} & =\frac{1}{3} q \dot{a}_{a}+\frac{1}{6} q R_{a 0}+\Phi_{a}^{\mathrm{tail}}+\mathcal{O}(r),
\end{aligned}
$$

and we see that $\Phi_{\alpha}^{\mathrm{R}}(x)$ is a smooth vector field on the world line. There is therefore no obstacle in evaluating the radiative field directly at $x=x^{\prime}$, where the tetrad $\left(e_{0}^{\alpha}, e_{a}^{\alpha}\right)$ becomes $\left(u^{\alpha^{\prime}}, e_{a}^{\alpha^{\prime}}\right)$. Reconstructing the field at $x^{\prime}$ from its frame components, we obtain

$$
\Phi_{\alpha^{\prime}}^{\mathrm{R}}\left(x^{\prime}\right)=-\frac{1}{12}(1-6 \xi) q R u_{\alpha^{\prime}}+q\left(g_{\alpha^{\prime} \beta^{\prime}}+u_{\alpha^{\prime}} u_{\beta^{\prime}}\right)\left(\frac{1}{3} \dot{a}^{\beta^{\prime}}+\frac{1}{6} R_{\gamma^{\prime}}^{\beta^{\prime}} u^{\gamma^{\prime}}\right)+\Phi_{\alpha^{\prime}}^{\mathrm{tail}},
$$

where the tail term can be copied from Equation (392),

$$
\Phi_{\alpha^{\prime}}^{\mathrm{tail}}\left(x^{\prime}\right)=q \int_{-\infty}^{u^{-}} \nabla_{\alpha^{\prime}} G_{+}\left(x^{\prime}, z\right) d \tau
$$

The tensors appearing in Equation (422) all refer to the retarded point $x^{\prime} \equiv z(u)$, which now stands for an arbitrary point on the world line $\gamma$.

\subsubsection{Equations of motion}

The retarded field $\Phi_{\alpha}(x)$ of a point scalar charge is singular on the world line, and this behaviour makes it difficult to understand how the field is supposed to act on the particle and affect its motion. The field's singularity structure was analyzed in Sections 5.1.3 and 5.1.4, and in Section 5.1.5 it was shown to originate from the singular field $\Phi_{\alpha}^{\mathrm{S}}(x)$; the radiative field $\Phi_{\alpha}^{\mathrm{R}}(x)=\Phi_{\alpha}(x)-\Phi_{\alpha}^{\mathrm{S}}(x)$ was then shown to be smooth on the world line.

To make sense of the retarded field's action on the particle we temporarily model the scalar charge not as a point particle, but as a small hollow shell that appears spherical when observed in a reference frame that is momentarily comoving with the particle; the shell's radius is $s_{0}$ in Fermi normal coordinates, and it is independent of the angles contained in the unit vector $\omega^{a}$. The net force acting at proper time $\tau$ on this hollow shell is the average of $q \Phi_{\alpha}\left(\tau, s_{0}, \omega^{a}\right)$ over the surface of the shell. This was worked out at the end of Section 5.1.4, and ignoring terms that disappear in the limit $s_{0} \rightarrow 0$, we obtain

$$
q\left\langle\Phi_{\mu}\right\rangle=-(\delta m) a_{\mu}-\frac{1}{12}(1-6 \xi) q^{2} R u_{\mu}+q^{2}\left(g_{\mu \nu}+u_{\mu} u_{\nu}\right)\left(\frac{1}{3} \dot{a}^{\nu}+\frac{1}{6} R_{\lambda}^{\nu} u^{\lambda}\right)+q \Phi_{\mu}^{\text {tail }}
$$

where

$$
\delta m \equiv \lim _{s_{0} \rightarrow 0} \frac{q^{2}}{3 s_{0}}
$$

is formally a divergent quantity and

$$
q \Phi_{\mu}^{\mathrm{tail}}=q^{2} \int_{-\infty}^{\tau^{-}} \nabla_{\mu} G_{+}\left(z(\tau), z\left(\tau^{\prime}\right)\right) d \tau^{\prime}
$$


is the tail part of the force; all tensors in Equation (424) are evaluated at an arbitrary point $z(\tau)$ on the world line.

Substituting Equations (424) and (426) into Equation (387) gives rise to the equations of motion

$$
(m+\delta m) a^{\mu}=q^{2}\left(\delta^{\mu}{ }_{\nu}+u^{\mu} u_{\nu}\right)\left[\frac{1}{3} \dot{a}^{\nu}+\frac{1}{6} R_{\lambda}^{\nu} u^{\lambda}+\int_{-\infty}^{\tau^{-}} \nabla^{\nu} G_{+}\left(z(\tau), z\left(\tau^{\prime}\right)\right) d \tau^{\prime}\right]
$$

for the scalar charge, with $m \equiv m_{0}-q \Phi(z)$ denoting the (also formally divergent) dynamical mass of the particle. We see that $m$ and $\delta m$ combine in Equation (427) to form the particle's observed mass $m_{\text {obs }}$, which is taken to be finite and to give a true measure of the particle's inertia. All diverging quantities have thus disappeared into the process of mass renormalization. Substituting Equations (424) and (426) into Equation (388), in which we replace $m$ by $m_{\mathrm{obs}}=m+\delta m$, returns an expression for the rate of change of the observed mass,

$$
\frac{d m_{\mathrm{obs}}}{d \tau}=-\frac{1}{12}(1-6 \xi) q^{2} R-q^{2} u^{\mu} \int_{-\infty}^{\tau^{-}} \nabla_{\mu} G_{+}\left(z(\tau), z\left(\tau^{\prime}\right)\right) d \tau^{\prime}
$$

That the observed mass is not conserved is a remarkable property of the dynamics of a scalar charge in a curved spacetime. Physically, this corresponds to the fact that in a spacetime with a time-dependent metric, a scalar charge radiates monopole waves and the radiated energy comes at the expense of the particle's inertial mass.

Apart from the term proportional to $\delta m$, the averaged field of Equation (424) has exactly the same form as the radiative field of Equation (422), which we re-express as

$$
q \Phi_{\mu}^{\mathrm{R}}=-\frac{1}{12}(1-6 \xi) q^{2} R u_{\mu}+q^{2}\left(g_{\mu \nu}+u_{\mu} u_{\nu}\right)\left(\frac{1}{3} \dot{a}^{\nu}+\frac{1}{6} R_{\lambda}^{\nu} u^{\lambda}\right)+q \Phi_{\mu}^{\mathrm{tail}} .
$$

The force acting on the point particle can therefore be thought of as originating from the (smooth) radiative field, while the singular field simply contributes to the particle's inertia. After mass renormalization, Equations (427) and (428) are equivalent to the statements

$$
m a^{\mu}=q\left(g^{\mu \nu}+u^{\mu} u^{\nu}\right) \Phi_{\nu}^{\mathrm{R}}(z), \quad \frac{d m}{d \tau}=-q u^{\mu} \Phi_{\mu}^{\mathrm{R}}(z)
$$

where we have dropped the superfluous label "obs" on the particle's observed mass. Another argument in support of the claim that the motion of the particle should be affected by the radiative field only was presented in Section 4.3.5.

The equations of motion displayed in Equations (427) and (428) are third-order differential equations for the functions $z^{\mu}(\tau)$. It is well known that such a system of equations admits many unphysical solutions, such as runaway situations in which the particle's acceleration increases exponentially with $\tau$, even in the absence of any external force [25, 30, 47]. And indeed, our equations of motion do not yet incorporate an external force which presumably is mostly responsible for the particle's acceleration. Both defects can be cured in one stroke. We shall take the point of view, the only admissible one in a classical treatment, that a point particle is merely an idealization for an extended object whose internal structure - the details of its charge distribution - can be considered to be irrelevant. This view automatically implies that our equations are meant to provide only an approximate description of the object's motion. It can then be shown [47, 26] that within the context of this approximation, it is consistent to replace, on the right-hand side of the equations of motion, any occurrence of the acceleration vector by $f_{\text {ext }}^{\mu} / m$, where $f_{\text {ext }}^{\mu}$ is the external force acting on the particle. Because $f_{\text {ext }}^{\mu}$ is a prescribed quantity, differentiation of the external force does not produce higher derivatives of the functions $z^{\mu}(\tau)$, and the equations of motion are properly of second order.

Living Reviews in Relativity

http: //www . livingreviews . org/Irr-2004-6 
We shall therefore write, in the final analysis, the equations of motion in the form

$$
m \frac{D u^{\mu}}{d \tau}=f_{\mathrm{ext}}^{\mu}+q^{2}\left(\delta^{\mu}{ }_{\nu}+u^{\mu} u_{\nu}\right)\left[\frac{1}{3 m} \frac{D f_{\mathrm{ext}}^{\nu}}{d \tau}+\frac{1}{6} R_{\lambda}^{\nu} u^{\lambda}+\int_{-\infty}^{\tau^{-}} \nabla^{\nu} G_{+}\left(z(\tau), z\left(\tau^{\prime}\right)\right) d \tau^{\prime}\right]
$$

and

$$
\frac{d m}{d \tau}=-\frac{1}{12}(1-6 \xi) q^{2} R-q^{2} u^{\mu} \int_{-\infty}^{\tau^{-}} \nabla_{\mu} G_{+}\left(z(\tau), z\left(\tau^{\prime}\right)\right) d \tau^{\prime},
$$

where $m$ denotes the observed inertial mass of the scalar charge, and where all tensors are evaluated at $z(\tau)$. We recall that the tail integration must be cut short at $\tau^{\prime}=\tau^{-} \equiv \tau-0^{+}$to avoid the singular behaviour of the retarded Green's function at coincidence; this procedure was justified at the beginning of Section 5.1.3. Equations (431) and (432) were first derived by Theodore C. Quinn in 2000 [48]. In his paper Quinn also establishes that the total work done by the scalar self-force matches the amount of energy radiated away by the particle.

\subsection{Motion of an electric charge}

\subsubsection{Dynamics of a point electric charge}

A point particle carries an electric charge $e$ and moves on a world line $\gamma$ described by relations $z^{\mu}(\lambda)$, in which $\lambda$ is an arbitrary parameter. The particle generates a vector potential $A^{\alpha}(x)$ and an electromagnetic field $F_{\alpha \beta}(x)=\nabla_{\alpha} A_{\beta}-\nabla_{\beta} A_{\alpha}$. The dynamics of the entire system is governed by the action

$$
S=S_{\text {field }}+S_{\text {particle }}+S_{\text {interaction }}
$$

where $S_{\text {field }}$ is an action functional for a free electromagnetic field in a spacetime with metric $g_{\alpha \beta}$, $S_{\text {particle }}$ is the action of a free particle moving on a world line $\gamma$ in this spacetime, and $S_{\text {interaction }}$ is an interaction term that couples the field to the particle.

The field action is given by

$$
S_{\text {field }}=-\frac{1}{16 \pi} \int F_{\alpha \beta} F^{\alpha \beta} \sqrt{-g} d^{4} x,
$$

where the integration is over all of spacetime. The particle action is

$$
S_{\text {particle }}=-m \int_{\gamma} d \tau
$$

where $m$ is the bare mass of the particle and $d \tau=\sqrt{-g_{\mu \nu}(z) \dot{z}^{\mu} \dot{z}^{\nu}} d \lambda$ is the differential of proper time along the world line; we use an overdot to indicate differentiation with respect to the parameter $\lambda$. Finally, the interaction term is given by

$$
S_{\text {interaction }}=e \int_{\gamma} A_{\mu}(z) \dot{z}^{\mu} d \lambda=e \int A_{\alpha}(x) g_{\mu}^{\alpha}(x, z) \dot{z}^{\mu} \delta_{4}(x, z) \sqrt{-g} d^{4} x d \lambda .
$$

Notice that both $S_{\text {particle }}$ and $S_{\text {interaction }}$ are invariant under a reparameterization $\lambda \rightarrow \lambda^{\prime}(\lambda)$ of the world line.

Demanding that the total action be stationary under a variation $\delta A^{\alpha}(x)$ of the vector potential yields Maxwell's equations

$$
F_{; \beta}^{\alpha \beta}=4 \pi j^{\alpha}
$$

with a current density $j^{\alpha}(x)$ defined by

$$
j^{\alpha}(x)=e \int_{\gamma} g_{\mu}^{\alpha}(x, z) \dot{z}^{\mu} \delta_{4}(x, z) d \lambda .
$$


These equations determine the electromagnetic field $F_{\alpha \beta}$, once the motion of the electric charge is specified. On the other hand, demanding that the total action be stationary under a variation $\delta z^{\mu}(\lambda)$ of the world line yields the equations of motion for the electric charge,

$$
m \frac{D u^{\mu}}{d \tau}=e F_{\nu}^{\mu}(z) u^{\nu} .
$$

We have adopted $\tau$ as the parameter on the world line, and introduced the four-velocity $u^{\mu}(\tau) \equiv$ $d z^{\mu} / d \tau$.

The electromagnetic field $F_{\alpha \beta}$ is invariant under a gauge transformation of the form $A_{\alpha} \rightarrow$ $A_{\alpha}+\nabla_{\alpha} \Lambda$, in which $\Lambda(x)$ is an arbitrary scalar function. This function can always be chosen so that the vector potential satisfies the Lorenz gauge condition,

$$
\nabla_{\alpha} A^{\alpha}=0
$$

Under this condition the Maxwell equations of Equation (437) reduce to a wave equation for the vector potential,

$$
\square A^{\alpha}-R_{\beta}^{\alpha} A^{\beta}=-4 \pi j^{\alpha},
$$

where $\square=g^{\alpha \beta} \nabla_{\alpha} \nabla_{\beta}$ is the wave operator and $R_{\beta}^{\alpha}$ is the Ricci tensor. Having adopted $\tau$ as the parameter on the world line, we can re-express the current density of Equation (438) as

$$
j^{\alpha}(x)=e \int_{\gamma} g_{\mu}^{\alpha}(x, z) u^{\mu} \delta_{4}(x, z) d \tau
$$

and we shall use Equations (441) and (442) to determine the electromagnetic field of a point electric charge. The motion of the particle is in principle determined by Equation (439), but because the vector potential obtained from Equation (441) is singular on the world line, these equations have only formal validity. Before we can make sense of them we will have to analyze the field's singularity structure near the world line. The calculations to be carried out parallel closely those presented in Section 5.1 for the case of a scalar charge; the details will therefore be kept to a minimum and the reader is referred to Section 5.1 for additional information.

\subsubsection{Retarded potential near the world line}

The retarded solution to Equation (441) is $A^{\alpha}(x)=\int G_{+\beta^{\prime}}^{\alpha}\left(x, x^{\prime}\right) j^{\beta^{\prime}}\left(x^{\prime}\right) \sqrt{g^{\prime}} d^{4} x^{\prime}$, where $G_{+\beta^{\prime}}^{\alpha}\left(x, x^{\prime}\right)$ is the retarded Green's function introduced in Section 4.4. After substitution of Equation (442) we obtain

$$
A^{\alpha}(x)=e \int_{\gamma} G_{+\mu}^{\alpha}(x, z) u^{\mu} d \tau
$$

in which $z^{\mu}(\tau)$ gives the description of the world line $\gamma$ and $u^{\mu}(\tau)=d z^{\mu} / d \tau$. Because the retarded Green's function is defined globally in the entire spacetime, Equation (443) applies to any field point $x$.

We now specialize Equation (443) to a point $x$ close to the world line. We let $\mathcal{N}(x)$ be the normal convex neighbourhood of this point, and we assume that the world line traverses $\mathcal{N}(x)$ (refer back to Figure 9). As in Section 5.1.2 we let $\tau_{<}$and $\tau_{>}$be the values of the proper-time parameter at which $\gamma$ enters and leaves $\mathcal{N}(x)$, respectively. Then Equation (443) can be expressed as

$$
A^{\alpha}(x)=e \int_{-\infty}^{\tau_{<}} G_{+\mu}^{\alpha}(x, z) u^{\mu} d \tau+e \int_{\tau_{<}}^{\tau_{>}} G_{+\mu}^{\alpha}(x, z) u^{\mu} d \tau+e \int_{\tau_{>}}^{\infty} G_{+\mu}^{\alpha}(x, z) u^{\mu} d \tau .
$$

Living Reviews in Relativity

http://www. livingreviews . org/lrr-2004-6 
The third integration vanishes because $x$ is then in the past of $z(\tau)$, and $G_{+\mu}^{\alpha}(x, z)=0$. For the second integration, $x$ is the normal convex neighbourhood of $z(\tau)$, and the retarded Green's function can be expressed in the Hadamard form produced in Section 4.4.2. This gives

$$
\int_{\tau_{<}}^{\tau_{>}} G_{+\mu}^{\alpha}(x, z) u^{\mu} d \tau=\int_{\tau_{<}}^{\tau_{>}} U_{\mu}^{\alpha}(x, z) u^{\mu} \delta_{+}(\sigma) d \tau+\int_{\tau_{<}}^{\tau_{>}} V_{\mu}^{\alpha}(x, z) u^{\mu} \theta_{+}(-\sigma) d \tau,
$$

and to evaluate this we let $x^{\prime} \equiv z(u)$ be the retarded point associated with $x$; these points are related by $\sigma\left(x, x^{\prime}\right)=0$ and $r \equiv \sigma_{\alpha^{\prime}} u^{\alpha^{\prime}}$ is the retarded distance between $x$ and the world line. To perform the first integration we change variables from $\tau$ to $\sigma$, noticing that $\sigma$ increases as $z(\tau)$ passes through $x^{\prime}$; the integral evaluates to $U_{\beta^{\prime}}^{\alpha} u^{\beta^{\prime}} / r$. The second integration is cut off at $\tau=u$ by the step function, and we obtain our final expression for the vector potential of a point electric charge:

$$
A^{\alpha}(x)=\frac{e}{r} U_{\beta^{\prime}}^{\alpha}\left(x, x^{\prime}\right) u^{\beta^{\prime}}+e \int_{\tau_{<}}^{u} V_{\mu}^{\alpha}(x, z) u^{\mu} d \tau+e \int_{-\infty}^{\tau_{<}} G_{+\mu}^{\alpha}(x, z) u^{\mu} d \tau .
$$

This expression applies to a point $x$ sufficiently close to the world line that there exists a nonempty intersection between $\mathcal{N}(x)$ and $\gamma$.

\subsubsection{Electromagnetic field in retarded coordinates}

When we differentiate the vector potential of Equation (444) we must keep in mind that a variation in $x$ induces a variation in $x^{\prime}$, because the new points $x+\delta x$ and $x^{\prime}+\delta x^{\prime}$ must also be linked by a null geodesic. Taking this into account, we find that the gradient of the vector potential is given by

$$
\begin{aligned}
& \nabla_{\beta} A_{\alpha}(x)= \\
& \quad-\frac{e}{r^{2}} U_{\alpha \beta^{\prime}} u^{\beta^{\prime}} \partial_{\beta} r+\frac{e}{r} U_{\alpha \beta^{\prime} ; \beta} u^{\beta^{\prime}}+\frac{e}{r}\left(U_{\alpha \beta^{\prime} ; \gamma^{\prime}} u^{\beta^{\prime}} u^{\gamma^{\prime}}+U_{\alpha \beta^{\prime}} a^{\beta^{\prime}}\right) \partial_{\beta} u+e V_{\alpha \beta^{\prime}} u^{\beta^{\prime}} \partial_{\beta} u+A_{\alpha \beta}^{\mathrm{tail}}(x),
\end{aligned}
$$

where the "tail integral" is defined by

$$
\begin{aligned}
A_{\alpha \beta}^{\text {tail }}(x) & =e \int_{\tau_{<}}^{u} \nabla_{\beta} V_{\alpha \mu}(x, z) u^{\mu} d \tau+e \int_{-\infty}^{\tau_{<}} \nabla_{\beta} G_{+\alpha \mu}(x, z) u^{\mu} d \tau \\
& =e \int_{-\infty}^{u^{-}} \nabla_{\beta} G_{+\alpha \mu}(x, z) u^{\mu} d \tau .
\end{aligned}
$$

The second form of the definition, in which we integrate the gradient of the retarded Green's function from $\tau=-\infty$ to $\tau=u^{-} \equiv u-0^{+}$to avoid the singular behaviour of the retarded Green's function at $\sigma=0$, is equivalent to the first form.

We shall now expand $F_{\alpha \beta}=\nabla_{\alpha} A_{\beta}-\nabla_{\beta} A_{\alpha}$ in powers of $r$, and express the result in terms of the retarded coordinates $\left(u, r, \Omega^{a}\right)$ introduced in Section 3.3. It will be convenient to decompose the electromagnetic field in the tetrad $\left(e_{0}^{\alpha}, e_{a}^{\alpha}\right)$ that is obtained by parallel transport of $\left(u^{\alpha^{\prime}}, e_{a}^{\alpha^{\prime}}\right)$ on the null geodesic that links $x$ to $x^{\prime} \equiv z(u)$; this construction is detailed in Section 3.3. Note that throughout this section we set $\omega_{a b}=0$, where $\omega_{a b}$ is the rotation tensor defined by Equation (138): The tetrad vectors $e_{a}^{\alpha^{\prime}}$ are taken to be Fermi-Walker transported on $\gamma$. We recall from Equation (141) that the parallel propagator can be expressed as $g_{\alpha}^{\alpha^{\prime}}=u^{\alpha^{\prime}} e_{\alpha}^{0}+e_{a}^{\alpha^{\prime}} e_{\alpha}^{a}$. The expansion relies on Equation (166) for $\partial_{\alpha} u$, Equation (168) for $\partial_{\alpha} r$, and we shall need

$$
U_{\alpha \beta^{\prime}} u^{\beta^{\prime}}=g_{\alpha}^{\alpha^{\prime}}\left[u_{\alpha^{\prime}}+\frac{1}{12} r^{2}\left(R_{00}+2 R_{0 a} \Omega^{a}+R_{a b} \Omega^{a} \Omega^{b}\right) u_{\alpha^{\prime}}+\mathcal{O}\left(r^{3}\right)\right],
$$


which follows from Equation (323) and the relation $\sigma^{\alpha^{\prime}}=-r\left(u^{\alpha^{\prime}}+\Omega^{a} e_{a}^{\alpha^{\prime}}\right)$ first encountered in Equation (144). We shall also need the expansions

$$
U_{\alpha \beta^{\prime} ; \beta} u^{\beta^{\prime}}=-\frac{1}{2} r g_{\alpha}^{\alpha^{\prime}} g_{\beta}^{\beta^{\prime}}\left[R_{\alpha^{\prime} 0 \beta^{\prime} 0}+R_{\alpha^{\prime} 0 \beta^{\prime} c} \Omega^{c}-\frac{1}{3}\left(R_{\beta^{\prime} 0}+R_{\beta^{\prime} c} \Omega^{c}\right) u_{\alpha^{\prime}}+\mathcal{O}(r)\right]
$$

and

$$
U_{\alpha \beta^{\prime} ; \gamma^{\prime}} u^{\beta^{\prime}} u^{\gamma^{\prime}}+U_{\alpha \beta^{\prime}} a^{\beta^{\prime}}=g_{\alpha}^{\alpha^{\prime}}\left[a_{\alpha^{\prime}}+\frac{1}{2} r R_{\alpha^{\prime} 0 b 0} \Omega^{b}-\frac{1}{6} r\left(R_{00}+R_{0 b} \Omega^{b}\right) u_{\alpha^{\prime}}+\mathcal{O}\left(r^{2}\right)\right]
$$

that follow from Equations (323, 324, 325). And finally, we shall need

$$
V_{\alpha \beta^{\prime}} u^{\beta^{\prime}}=-\frac{1}{2} g_{\alpha}^{\alpha^{\prime}}\left[R_{\alpha^{\prime} 0}-\frac{1}{6} R u_{\alpha^{\prime}}+\mathcal{O}(r)\right],
$$

a relation that was first established in Equation (327).

Collecting all these results gives

$$
\begin{aligned}
F_{a 0}\left(u, r, \Omega^{a}\right) \equiv & F_{\alpha \beta}(x) e_{a}^{\alpha}(x) e_{0}^{\beta}(x) \\
= & \frac{e}{r^{2}} \Omega_{a}-\frac{e}{r}\left(a_{a}-a_{b} \Omega^{b} \Omega_{a}\right)+\frac{1}{3} e R_{b 0 c 0} \Omega^{b} \Omega^{c} \Omega_{a}-\frac{1}{6} e\left(5 R_{a 0 b 0} \Omega^{b}+R_{a b 0 c} \Omega^{b} \Omega^{c}\right) \\
& +\frac{1}{12} e\left(5 R_{00}+R_{b c} \Omega^{b} \Omega^{c}+R\right) \Omega_{a}+\frac{1}{3} e R_{a 0}-\frac{1}{6} e R_{a b} \Omega^{b}+F_{a 0}^{\mathrm{tail}}+\mathcal{O}(r), \\
F_{a b}\left(u, r, \Omega^{a}\right) \equiv & F_{\alpha \beta}(x) e_{a}^{\alpha}(x) e_{b}^{\beta}(x) \\
= & \frac{e}{r}\left(a_{a} \Omega_{b}-\Omega_{a} a_{b}\right)+\frac{1}{2} e\left(R_{a 0 b c}-R_{b 0 a c}+R_{a 0 c 0} \Omega_{b}-\Omega_{a} R_{b 0 c 0}\right) \Omega^{c} \\
& -\frac{1}{2} e\left(R_{a 0} \Omega_{b}-\Omega_{a} R_{b 0}\right)+F_{a b}^{\mathrm{tail}}+\mathcal{O}(r),
\end{aligned}
$$

where

$$
F_{a 0}^{\mathrm{tail}}=F_{\alpha^{\prime} \beta^{\prime}}^{\mathrm{tail}}\left(x^{\prime}\right) e_{a}^{\alpha^{\prime}} u^{\beta^{\prime}}, \quad F_{a b}^{\mathrm{tail}}=F_{\alpha^{\prime} \beta^{\prime}}^{\mathrm{tail}}\left(x^{\prime}\right) e_{a}^{\alpha^{\prime}} e_{b}^{\beta^{\prime}}
$$

are the frame components of the tail integral; this is obtained from Equation (446) evaluated at $x^{\prime}$ :

$$
F_{\alpha^{\prime} \beta^{\prime}}^{\text {tail }}\left(x^{\prime}\right)=2 e \int_{-\infty}^{u^{-}} \nabla_{\left[\alpha^{\prime}\right.} G_{\left.+\beta^{\prime}\right] \mu}\left(x^{\prime}, z\right) u^{\mu} d \tau .
$$

It should be emphasized that in Equations (451) and (452), all frame components are evaluated at the retarded point $x^{\prime} \equiv z(u)$ associated with $x$; for example, $a_{a} \equiv a_{a}(u) \equiv a_{\alpha^{\prime}} e_{a}^{\alpha^{\prime}}$. It is clear from these equations that the electromagnetic field $F_{\alpha \beta}(x)$ is singular on the world line.

\subsubsection{Electromagnetic field in Fermi normal coordinates}

We now wish to express the electromagnetic field in the Fermi normal coordinates of Section 3.2; as before those will be denoted $\left(t, s, \omega^{a}\right)$. The translation will be carried out as in Section 5.1.4, and we will decompose the field in the tetrad $\left(\bar{e}_{0}^{\alpha}, \bar{e}_{a}^{\alpha}\right)$ that is obtained by parallel transport of $\left(u^{\bar{\alpha}}, e_{a}^{\bar{\alpha}}\right)$ on the spacelike geodesic that links $x$ to the simultaneous point $\bar{x} \equiv z(t)$.

Our first task is to decompose $F_{\alpha \beta}(x)$ in the tetrad $\left(\bar{e}_{0}^{\alpha}, \bar{e}_{a}^{\alpha}\right)$, thereby defining $\bar{F}_{a 0} \equiv F_{\alpha \beta} \bar{e}_{a}^{\alpha} \bar{e}_{0}^{\beta}$ and $\bar{F}_{a b} \equiv F_{\alpha \beta} \bar{e}_{a}^{\alpha} \bar{e}_{b}^{\beta}$. For this purpose we use Equations $(224,225)$ and $(451,452)$ to obtain

$$
\begin{aligned}
\bar{F}_{a 0}= & \frac{e}{r^{2}} \Omega_{a}-\frac{e}{r}\left(a_{a}-a_{b} \Omega^{b} \Omega_{a}\right)+\frac{1}{2} e a_{b} \Omega^{b} a_{a}+\frac{1}{2} e \dot{a}_{0} \Omega_{a}-\frac{5}{6} e R_{a 0 b 0} \Omega^{b}+\frac{1}{3} e R_{b 0 c 0} \Omega^{b} \Omega^{c} \Omega_{a} \\
& +\frac{1}{3} e R_{a b 0 c} \Omega^{b} \Omega^{c}+\frac{1}{12} e\left(5 R_{00}+R_{b c} \Omega^{b} \Omega^{c}+R\right) \Omega_{a}+\frac{1}{3} e R_{a 0}-\frac{1}{6} e R_{a b} \Omega^{b}+\bar{F}_{a 0}^{\text {tail }}+\mathcal{O}(r)
\end{aligned}
$$

Living Reviews in Relativity

http://www. livingreviews . org//rr-2004-6 
and

$$
\bar{F}_{a b}=\frac{1}{2} e\left(\Omega_{a} \dot{a}_{b}-\dot{a}_{a} \Omega_{b}\right)+\frac{1}{2} e\left(R_{a 0 b c}-R_{b 0 a c}\right) \Omega^{c}-\frac{1}{2} e\left(R_{a 0} \Omega_{b}-\Omega_{a} R_{b 0}\right)+\bar{F}_{a b}^{\text {tail }}+\mathcal{O}(r),
$$

where all frame components are still evaluated at $x^{\prime}$, except for

$$
\bar{F}_{a 0}^{\text {tail }} \equiv F_{\bar{\alpha} \bar{\beta}}^{\text {tail }}(\bar{x}) e_{a}^{\bar{\alpha}} u^{\bar{\beta}}, \quad \bar{F}_{a b}^{\text {tail }} \equiv F_{\bar{\alpha} \bar{\beta}}^{\text {tail }}(\bar{x}) e_{a}^{\bar{\alpha}} e_{b}^{\bar{\beta}},
$$

which are evaluated at $\bar{x}$.

We must still translate these results into the Fermi normal coordinates $\left(t, s, \omega^{a}\right)$. For this we involve Equations $(221,222,223)$, and we recycle some computations that were first carried out in Section 5.1.4. After some algebra, we arrive at

$$
\begin{aligned}
\bar{F}_{a 0}\left(t, s, \omega^{a}\right) \equiv & F_{\alpha \beta}(x) \bar{e}_{a}^{\alpha}(x) \bar{e}_{0}^{\beta}(x) \\
= & \frac{e}{s^{2}} \omega_{a}-\frac{e}{2 s}\left(a_{a}+a_{b} \omega^{b} \omega_{a}\right)+\frac{3}{4} e a_{b} \omega^{b} a_{a}+\frac{3}{8} e\left(a_{b} \omega^{b}\right)^{2} \omega_{a}+\frac{3}{8} e \dot{a}_{0} \omega_{a}+\frac{2}{3} e \dot{a}_{a} \\
& -\frac{2}{3} e R_{a 0 b 0} \omega^{b}-\frac{1}{6} e R_{b 0 c 0} \omega^{b} \omega^{c} \omega_{a}+\frac{1}{12} e\left(5 R_{00}+R_{b c} \omega^{b} \omega^{c}+R\right) \omega_{a} \\
& +\frac{1}{3} e R_{a 0}-\frac{1}{6} e R_{a b} \omega^{b}+\bar{F}_{a 0}^{\text {tail }}+\mathcal{O}(s), \\
\bar{F}_{a b}\left(t, s, \omega^{a}\right) \equiv & F_{\alpha \beta}(x) \bar{e}_{a}^{\alpha}(x) \bar{e}_{b}^{\beta}(x) \\
= & \frac{1}{2} e\left(\omega_{a} \dot{a}_{b}-\dot{a}_{a} \omega_{b}\right)+\frac{1}{2} e\left(R_{a 0 b c}-R_{b 0 a c}\right) \omega^{c}-\frac{1}{2} e\left(R_{a 0} \omega_{b}-\omega_{a} R_{b 0}\right)+\bar{F}_{a b}^{\text {tail }}+\mathcal{O}(s),
\end{aligned}
$$

where all frame components are now evaluated at $\bar{x} \equiv z(t)$; for example, $a_{a} \equiv a_{a}(t) \equiv a_{\bar{\alpha}} e_{a}^{\bar{\alpha}}$.

Our next task is to compute the averages of $\bar{F}_{a 0}$ and $\bar{F}_{a b}$ over $S(t, s)$, a two-surface of constant $t$ and $s$. These are defined by

$$
\left\langle\bar{F}_{a 0}\right\rangle(t, s)=\frac{1}{\mathcal{A}} \oint_{S(t, s)} \bar{F}_{a 0}\left(t, s, \omega^{a}\right) d \mathcal{A}, \quad\left\langle\bar{F}_{a b}\right\rangle(t, s)=\frac{1}{\mathcal{A}} \oint_{S(t, s)} \bar{F}_{a b}\left(t, s, \omega^{a}\right) d \mathcal{A},
$$

where $d \mathcal{A}$ is the element of surface area on $S(t, s)$, and $\mathcal{A}=\oint d \mathcal{A}$. Using the methods developed in Section 5.1.4, we find

$$
\begin{aligned}
\left\langle\bar{F}_{a 0}\right\rangle & =-\frac{2 e}{3 s} a_{a}+\frac{2}{3} e \dot{a}_{a}+\frac{1}{3} e R_{a 0}+\bar{F}_{a 0}^{\text {tail }}+\mathcal{O}(s), \\
\left\langle\bar{F}_{a b}\right\rangle & =\bar{F}_{a b}^{\text {tail }}+\mathcal{O}(s) .
\end{aligned}
$$

The averaged field is singular on the world line, but we nevertheless take the formal limit $s \rightarrow 0$ of the expressions displayed in Equations (458) and (459). In the limit the tetrad $\left(\bar{e}_{0}^{\alpha}, \bar{e}_{a}^{\alpha}\right)$ becomes $\left(u^{\bar{\alpha}}, e_{a}^{\bar{\alpha}}\right)$, and we can easily reconstruct the field at $\bar{x}$ from its frame components. We thus obtain

$$
\left\langle F_{\bar{\alpha} \bar{\beta}}\right\rangle=\lim _{s \rightarrow 0}\left(-\frac{4 e}{3 s}\right) u_{[\bar{\alpha}} a_{\bar{\beta}]}+2 e u_{[\bar{\alpha}}\left(g_{\bar{\beta}] \bar{\gamma}}+u_{\bar{\beta}]} u_{\bar{\gamma}}\right)\left(\frac{2}{3} \dot{a}^{\bar{\gamma}}+\frac{1}{3} R_{\bar{\delta}}^{\bar{\gamma}} u^{\bar{\delta}}\right)+F_{\bar{\alpha} \bar{\beta}}^{\text {tail }},
$$

where the tail term can be copied from Equation (454),

$$
F_{\bar{\alpha} \bar{\beta}}^{\mathrm{tail}}(\bar{x})=2 e \int_{-\infty}^{t^{-}} \nabla_{[\bar{\alpha}} G_{+\bar{\beta}] \mu}(\bar{x}, z) u^{\mu} d \tau .
$$

The tensors appearing in Equation (460) all refer to $\bar{x} \equiv z(t)$, which now stands for an arbitrary point on the world line $\gamma$. 


\subsubsection{Singular and radiative fields}

The singular vector potential

$$
A_{\mathrm{S}}^{\alpha}(x)=e \int_{\gamma} G_{\mathrm{S} \mu}^{\alpha}(x, z) u^{\mu} d \tau
$$

is the (unphysical) solution to Equations (441) and (442) that is obtained by adopting the singular Green's function of Equation (335) instead of the retarded Green's function. We will see that the singular field $F_{\alpha \beta}^{\mathrm{S}}$ reproduces the singular behaviour of the retarded solution, and that the difference, $F_{\alpha \beta}^{\mathrm{R}}=F_{\alpha \beta}-F_{\alpha \beta}^{\mathrm{S}}$, is smooth on the world line.

To evaluate the integral of Equation (462) we assume once more that $x$ is sufficiently close to $\gamma$ that the world line traverses $\mathcal{N}(x)$ (refer back to Figure 9). As before we let $\tau_{<}$and $\tau_{>}$be the values of the proper-time parameter at which $\gamma$ enters and leaves $\mathcal{N}(x)$, respectively. Then Equation (462) becomes

$$
A_{\mathrm{S}}^{\alpha}(x)=e \int_{-\infty}^{\tau_{<}} G_{\mathrm{S} \mu}^{\alpha}(x, z) u^{\mu} d \tau+e \int_{\tau_{<}}^{\tau_{>}} G_{\mathrm{S} \mu}^{\alpha}(x, z) u^{\mu} d \tau+e \int_{\tau_{>}}^{\infty} G_{\mathrm{S} \mu}^{\alpha}(x, z) u^{\mu} d \tau .
$$

The first integration vanishes because $x$ is then in the chronological future of $z(\tau)$, and $G_{\mathrm{S} \mu}^{\alpha}(x, z)=$ 0 by Equation (338). Similarly, the third integration vanishes because $x$ is then in the chronological past of $z(\tau)$. For the second integration, $x$ is the normal convex neighbourhood of $z(\tau)$, the singular Green's function can be expressed in the Hadamard form of Equation (344), and we have

$$
\begin{aligned}
& \int_{\tau_{<}}^{\tau_{>}} G_{\mathrm{S} \mu}^{\alpha}(x, z) u^{\mu} d \tau= \\
& \quad \frac{1}{2} \int_{\tau_{<}}^{\tau_{>}} U_{\mu}^{\alpha}(x, z) u^{\mu} \delta_{+}(\sigma) d \tau+\frac{1}{2} \int_{\tau_{<}}^{\tau_{>}} U_{\mu}^{\alpha}(x, z) u^{\mu} \delta_{-}(\sigma) d \tau-\frac{1}{2} \int_{\tau_{<}}^{\tau_{>}} V_{\mu}^{\alpha}(x, z) u^{\mu} \theta(\sigma) d \tau .
\end{aligned}
$$

To evaluate these we let $x^{\prime} \equiv z(u)$ and $x^{\prime \prime} \equiv z(v)$ be the retarded and advanced points associated with $x$, respectively. To perform the first integration we change variables from $\tau$ to $\sigma$, noticing that $\sigma$ increases as $z(\tau)$ passes through $x^{\prime}$; the integral evaluates to $U_{\beta^{\prime}}^{\alpha} u^{\beta^{\prime}} / r$. We do the same for the second integration, but we notice now that $\sigma$ decreases as $z(\tau)$ passes through $x^{\prime \prime}$; the integral evaluates to $U_{\beta^{\prime \prime}}^{\alpha} u^{\beta^{\prime \prime}} / r_{\mathrm{adv}}$, where $r_{\mathrm{adv}} \equiv-\sigma_{\alpha^{\prime \prime}} u^{\alpha^{\prime \prime}}$ is the advanced distance between $x$ and the world line. The third integration is restricted to the interval $u \leq \tau \leq v$ by the step function, and we obtain the expression

$$
A_{\mathrm{S}}^{\alpha}(x)=\frac{e}{2 r} U_{\beta^{\prime}}^{\alpha} u^{\beta^{\prime}}+\frac{e}{2 r_{\mathrm{adv}}} U_{\beta^{\prime \prime}}^{\alpha} u^{\beta^{\prime \prime}}-\frac{1}{2} e \int_{u}^{v} V_{\mu}^{\alpha}(x, z) u^{\mu} d \tau
$$

for the singular vector potential.

Differentiation of Equation (463) yields

$$
\begin{aligned}
\nabla_{\beta} A_{\alpha}^{\mathrm{S}}(x)= & -\frac{e}{2 r^{2}} U_{\alpha \beta^{\prime}} u^{\beta^{\prime}} \partial_{\beta} r-\frac{e}{2 r_{\mathrm{adv}}{ }^{2}} U_{\alpha \beta^{\prime \prime}} u^{\beta^{\prime \prime}} \partial_{\beta} r_{\mathrm{adv}}+\frac{e}{2 r} U_{\alpha \beta^{\prime} ; \beta} u^{\beta^{\prime}} \\
& +\frac{e}{2 r}\left(U_{\alpha \beta^{\prime} ; \gamma^{\prime}} u^{\beta^{\prime}} u^{\gamma^{\prime}}+U_{\alpha \beta^{\prime}} a^{\beta^{\prime}}\right) \partial_{\beta} u+\frac{e}{2 r_{\mathrm{adv}}} U_{\alpha \beta^{\prime \prime} ; \beta} u^{\beta^{\prime \prime}} \\
& +\frac{e}{2 r_{\mathrm{adv}}}\left(U_{\alpha \beta^{\prime \prime} ; \gamma^{\prime \prime}} u^{\beta^{\prime \prime}} u^{\gamma^{\prime \prime}}+U_{\alpha \beta^{\prime \prime}} a^{\beta^{\prime \prime}}\right) \partial_{\beta} v+\frac{1}{2} e V_{\alpha \beta^{\prime}} u^{\beta^{\prime}} \partial_{\beta} u \\
& -\frac{1}{2} e V_{\alpha \beta^{\prime \prime}} u^{\beta^{\prime \prime}} \partial_{\beta} v-\frac{1}{2} e \int_{u}^{v} \nabla_{\beta} V_{\alpha \mu}(x, z) u^{\mu} d \tau
\end{aligned}
$$

and we would like to express this as an expansion in powers of $r$. For this we will rely on results already established in Section 5.2.3, as well as additional expansions that will involve the advanced

Living Reviews in Relativity

http://www. livingreviews . org//rr-2004-6 
point $x^{\prime \prime}$. We recall that a relation between retarded and advanced times was worked out in Equation (229), that an expression for the advanced distance was displayed in Equation (230), and that Equations (231) and (232) give expansions for $\partial_{\alpha} v$ and $\partial_{\alpha} r_{\text {adv }}$, respectively.

To derive an expansion for $U_{\alpha \beta^{\prime \prime}} u^{\beta^{\prime \prime}}$ we follow the general method of Section 3.4.4 and introduce the functions $U_{\alpha}(\tau) \equiv U_{\alpha \mu}(x, z) u^{\mu}$. We have that

$$
U_{\alpha \beta^{\prime \prime}} u^{\beta^{\prime \prime}} \equiv U_{\alpha}(v)=U_{\alpha}(u)+\dot{U}_{\alpha}(u) \Delta^{\prime}+\frac{1}{2} \ddot{U}_{\alpha}(u) \Delta^{\prime 2}+O\left(\Delta^{\prime 3}\right),
$$

where overdots indicate differentiation with respect to $\tau$, and $\Delta^{\prime} \equiv v-u$. The leading term $U_{\alpha}(u) \equiv U_{\alpha \beta^{\prime}} u^{\beta^{\prime}}$ was worked out in Equation (447), and the derivatives of $U_{\alpha}(\tau)$ are given by

$$
\dot{U}_{\alpha}(u)=U_{\alpha \beta^{\prime} ; \gamma^{\prime}} u^{\beta^{\prime}} u^{\gamma^{\prime}}+U_{\alpha \beta^{\prime}} a^{\beta^{\prime}}=g_{\alpha}^{\alpha^{\prime}}\left[a_{\alpha^{\prime}}+\frac{1}{2} r R_{\alpha^{\prime} 0 b 0} \Omega^{b}-\frac{1}{6} r\left(R_{00}+R_{0 b} \Omega^{b}\right) u_{\alpha^{\prime}}+\mathcal{O}\left(r^{2}\right)\right]
$$

and

$\ddot{U}_{\alpha}(u)=U_{\alpha \beta^{\prime} ; \gamma^{\prime} \delta^{\prime}} u^{\beta^{\prime}} u^{\gamma^{\prime}} u^{\delta^{\prime}}+U_{\alpha \beta^{\prime} ; \gamma^{\prime}}\left(2 a^{\beta^{\prime}} u^{\gamma^{\prime}}+u^{\beta^{\prime}} a^{\gamma^{\prime}}\right)+U_{\alpha \beta^{\prime}} \dot{a}^{\beta^{\prime}}=g_{\alpha}^{\alpha^{\prime}}\left[\dot{a}_{\alpha^{\prime}}+\frac{1}{6} R_{00} u_{\alpha^{\prime}}+\mathcal{O}(r)\right]$,

according to Equations (449) and (325). Combining these results together with Equation (229) for $\Delta^{\prime}$ gives

$$
\begin{aligned}
U_{\alpha \beta^{\prime \prime}} u^{\beta^{\prime \prime}}=g_{\alpha}^{\alpha^{\prime}} & {\left[u_{\alpha^{\prime}}+2 r\left(1-r a_{b} \Omega^{b}\right) a_{\alpha^{\prime}}+2 r^{2} \dot{a}_{\alpha^{\prime}}+r^{2} R_{\alpha^{\prime} 0 b 0} \Omega^{b}\right.} \\
& \left.+\frac{1}{12} r^{2}\left(R_{00}-2 R_{0 a} \Omega^{a}+R_{a b} \Omega^{a} \Omega^{b}\right) u_{\alpha^{\prime}}+\mathcal{O}\left(r^{3}\right)\right],
\end{aligned}
$$

which should be compared with Equation (447). It should be emphasized that in Equation (465) and all equations below, all frame components are evaluated at the retarded point $x^{\prime}$, and not at the advanced point. The preceding computation gives us also an expansion for

$$
U_{\alpha \beta^{\prime \prime} ; \gamma^{\prime \prime}} u^{\beta^{\prime \prime}} u^{\gamma^{\prime \prime}}+U_{\alpha \beta^{\prime \prime}} a^{\beta^{\prime \prime}} \equiv \dot{U}_{\alpha}(v)=\dot{U}_{\alpha}(u)+\ddot{U}_{\alpha}(u) \Delta^{\prime}+\mathcal{O}\left(\Delta^{\prime 2}\right),
$$

which becomes

$$
U_{\alpha \beta^{\prime \prime} ; \gamma^{\prime \prime}} u^{\beta^{\prime \prime}} u^{\gamma^{\prime \prime}}+U_{\alpha \beta^{\prime \prime}} a^{\beta^{\prime \prime}}=g_{\alpha}^{\alpha^{\prime}}\left[a_{\alpha^{\prime}}+2 r \dot{a}_{\alpha^{\prime}}+\frac{1}{2} r R_{\alpha^{\prime} 0 b 0} \Omega^{b}+\frac{1}{6} r\left(R_{00}-R_{0 b} \Omega^{b}\right) u_{\alpha^{\prime}}+\mathcal{O}\left(r^{2}\right)\right]
$$

and which should be compared with Equation (449).

We proceed similarly to derive an expansion for $U_{\alpha \beta^{\prime \prime} ; \beta} u^{\beta^{\prime \prime}}$. Here we introduce the functions $U_{\alpha \beta}(\tau) \equiv U_{\alpha \mu ; \beta} u^{\mu}$ and express $U_{\alpha \beta^{\prime \prime} ; \beta} u^{\beta^{\prime \prime}}$ as $U_{\alpha \beta}(v)=U_{\alpha \beta}(u)+\dot{U}_{\alpha \beta}(u) \Delta^{\prime}+\mathcal{O}\left(\Delta^{\prime 2}\right)$. The leading term $U_{\alpha \beta}(u) \equiv U_{\alpha \beta^{\prime} ; \beta} u^{\beta^{\prime}}$ was computed in Equation (448), and

$$
\dot{U}_{\alpha \beta}(u)=U_{\alpha \beta^{\prime} ; \beta \gamma^{\prime}} u^{\beta^{\prime}} u^{\gamma^{\prime}}+U_{\alpha \beta^{\prime} ; \beta} a^{\beta^{\prime}}=\frac{1}{2} g_{\alpha}^{\alpha^{\prime}} g_{\beta}^{\beta^{\prime}}\left[R_{\alpha^{\prime} 0 \beta^{\prime} 0}-\frac{1}{3} u_{\alpha^{\prime}} R_{\beta^{\prime} 0}+\mathcal{O}(r)\right]
$$

follows from Equation (324). Combining these results together with Equation (229) for $\Delta^{\prime}$ gives

$$
U_{\alpha \beta^{\prime \prime} ; \beta} u^{\beta^{\prime \prime}}=\frac{1}{2} r g_{\alpha}^{\alpha^{\prime}} g_{\beta}^{\beta^{\prime}}\left[R_{\alpha^{\prime} 0 \beta^{\prime} 0}-R_{\alpha^{\prime} 0 \beta^{\prime} c} \Omega^{c}-\frac{1}{3}\left(R_{\beta^{\prime} 0}-R_{\beta^{\prime} c} \Omega^{c}\right) u_{\alpha^{\prime}}+\mathcal{O}(r)\right],
$$


and this should be compared with Equation (448). The last expansion we shall need is

$$
V_{\alpha \beta^{\prime \prime}} u^{\beta^{\prime \prime}}=-\frac{1}{2} g_{\alpha}^{\alpha^{\prime}}\left[R_{\alpha^{\prime} 0}-\frac{1}{6} R u_{\alpha^{\prime}}+\mathcal{O}(r)\right]
$$

which follows at once from Equation (450).

It is now a straightforward (but still tedious) matter to substitute these expansions into Equation (464) to obtain the projections of the singular electromagnetic field $F_{\alpha \beta}^{\mathrm{S}}=\nabla_{\alpha} A_{\beta}^{\mathrm{S}}-\nabla_{\beta} A_{\alpha}^{\mathrm{S}}$ in the same tetrad $\left(e_{0}^{\alpha}, e_{a}^{\alpha}\right)$ that was employed in Section 5.2.3. This gives

$$
\begin{aligned}
F_{a 0}^{\mathrm{S}}\left(u, r, \Omega^{a}\right) \equiv & F_{\alpha \beta}^{\mathrm{S}}(x) e_{a}^{\alpha}(x) e_{0}^{\beta}(x) \\
= & \frac{e}{r^{2}} \Omega_{a}-\frac{e}{r}\left(a_{a}-a_{b} \Omega^{b} \Omega_{a}\right)-\frac{2}{3} e \dot{a}_{a}+\frac{1}{3} e R_{b 0 c 0} \Omega^{b} \Omega^{c} \Omega_{a}-\frac{1}{6} e\left(5 R_{a 0 b 0} \Omega^{b}+R_{a b 0 c} \Omega^{b} \Omega^{c}\right) \\
& +\frac{1}{12} e\left(5 R_{00}+R_{b c} \Omega^{b} \Omega^{c}+R\right) \Omega_{a}-\frac{1}{6} e R_{a b} \Omega^{b}+\mathcal{O}(r), \\
F_{a b}^{\mathrm{S}}\left(u, r, \Omega^{a}\right) \equiv & F_{\alpha \beta}^{\mathrm{S}}(x) e_{a}^{\alpha}(x) e_{b}^{\beta}(x) \\
= & \frac{e}{r}\left(a_{a} \Omega_{b}-\Omega_{a} a_{b}\right)+\frac{1}{2} e\left(R_{a 0 b c}-R_{b 0 a c}+R_{a 0 c 0} \Omega_{b}-\Omega_{a} R_{b 0 c 0}\right) \Omega^{c} \\
& -\frac{1}{2} e\left(R_{a 0} \Omega_{b}-\Omega_{a} R_{b 0}\right)+\mathcal{O}(r),
\end{aligned}
$$

in which all frame components are evaluated at the retarded point $x^{\prime}$. Comparison of these expressions with Equations (451) and (452) reveals that the retarded and singular fields share the same singularity structure.

The difference between the retarded field of Equations $(451,452)$ and the singular field of Equations $(469,470)$ defines the radiative field $F_{\alpha \beta}^{\mathrm{R}}(x)$. Its tetrad components are

$$
\begin{aligned}
F_{a 0}^{\mathrm{R}} & =\frac{2}{3} e \dot{a}_{a}+\frac{1}{3} e R_{a 0}+F_{a 0}^{\mathrm{tail}}+\mathcal{O}(r), \\
F_{a b}^{\mathrm{R}} & =F_{a b}^{\mathrm{tail}}+\mathcal{O}(r),
\end{aligned}
$$

and we see that $F_{\alpha \beta}^{\mathrm{R}}$ is a smooth tensor field on the world line. There is therefore no obstacle in evaluating the radiative field directly at $x=x^{\prime}$, where the tetrad $\left(e_{0}^{\alpha}, e_{a}^{\alpha}\right)$ becomes $\left(u^{\alpha^{\prime}}, e_{a}^{\alpha^{\prime}}\right)$. Reconstructing the field at $x^{\prime}$ from its frame components, we obtain

$$
F_{\alpha^{\prime} \beta^{\prime}}^{\mathrm{R}}\left(x^{\prime}\right)=2 e u_{\left[\alpha^{\prime}\right.}\left(g_{\left.\beta^{\prime}\right] \gamma^{\prime}}+u_{\left.\beta^{\prime}\right]} u_{\gamma^{\prime}}\right)\left(\frac{2}{3} \dot{a}^{\gamma^{\prime}}+\frac{1}{3} R_{\delta^{\prime}}^{\gamma^{\prime}} u^{\delta^{\prime}}\right)+F_{\alpha^{\prime} \beta^{\prime}}^{\text {tail }},
$$

where the tail term can be copied from Equation (454),

$$
F_{\alpha^{\prime} \beta^{\prime}}^{\mathrm{tail}}\left(x^{\prime}\right)=2 e \int_{-\infty}^{u^{-}} \nabla_{\left[\alpha^{\prime}\right.} G_{\left.+\beta^{\prime}\right] \mu}\left(x^{\prime}, z\right) u^{\mu} d \tau .
$$

The tensors appearing in Equation (473) all refer to the retarded point $x^{\prime} \equiv z(u)$, which now stands for an arbitrary point on the world line $\gamma$.

\subsubsection{Equations of motion}

The retarded field $F_{\alpha \beta}$ of a point electric charge is singular on the world line, and this behaviour makes it difficult to understand how the field is supposed to act on the particle and exert a force. The field's singularity structure was analyzed in Sections 5.2.3 and 5.2.4, and in Section 5.2.5 it

Living Reviews in Relativity

http://www. livingreviews.org/lrr-2004-6 
was shown to originate from the singular field $F_{\alpha \beta}^{\mathrm{S}}$; the radiative field $F_{\alpha \beta}^{\mathrm{R}}=F_{\alpha \beta}-F_{\alpha \beta}^{\mathrm{S}}$ was then shown to be smooth on the world line.

To make sense of the retarded field's action on the particle we follow the discussion of Section 5.1.6 and temporarily picture the electric charge as a spherical hollow shell; the shell's radius is $s_{0}$ in Fermi normal coordinates, and it is independent of the angles contained in the unit vector $\omega^{a}$. The net force acting at proper time $\tau$ on this shell is proportional to the average of $F_{\alpha \beta}\left(\tau, s_{0}, \omega^{a}\right)$ over the shell's surface. This was worked out at the end of Section 5.2.4, and ignoring terms that disappear in the limit $s_{0} \rightarrow 0$, we obtain

$$
e\left\langle F_{\mu \nu}\right\rangle u^{\nu}=-(\delta m) a_{\mu}+e^{2}\left(g_{\mu \nu}+u_{\mu} u_{\nu}\right)\left(\frac{2}{3} \dot{a}^{\nu}+\frac{1}{3} R_{\lambda}^{\nu} u^{\lambda}\right)+e F_{\mu \nu}^{\text {tail }} u^{\nu},
$$

where

$$
\delta m \equiv \lim _{s_{0} \rightarrow 0} \frac{2 e^{2}}{3 s_{0}}
$$

is formally a divergent quantity and

$$
e F_{\mu \nu}^{\text {tail }} u^{\nu}=2 e^{2} u^{\nu} \int_{-\infty}^{\tau^{-}} \nabla_{[\mu} G_{+\nu] \lambda^{\prime}}\left(z(\tau), z\left(\tau^{\prime}\right)\right) u^{\lambda^{\prime}} d \tau^{\prime}
$$

is the tail part of the force; all tensors in Equation (475) are evaluated at an arbitrary point $z(\tau)$ on the world line.

Substituting Equations (475) and (477) into Equation (439) gives rise to the equations of motion for the electric charge

$$
(m+\delta m) a^{\mu}=e^{2}\left(\delta^{\mu}{ }_{\nu}+u^{\mu} u_{\nu}\right)\left(\frac{2}{3} \dot{a}^{\nu}+\frac{1}{3} R_{\lambda}^{\nu} u^{\lambda}\right)+2 e^{2} u_{\nu} \int_{-\infty}^{\tau^{-}} \nabla^{[\mu} G_{+\lambda^{\prime}}^{\nu]}\left(z(\tau), z\left(\tau^{\prime}\right)\right) u^{\lambda^{\prime}} d \tau^{\prime}
$$

with $m$ denoting the (also formally divergent) bare mass of the particle. We see that $m$ and $\delta m$ combine in Equation (478) to form the particle's observed mass $m_{\mathrm{obs}}$, which is finite and gives a true measure of the particle's inertia. All diverging quantities have thus disappeared into the procedure of mass renormalization.

Apart from the term proportional to $\delta m$, the averaged force of Equation (475) has exactly the same form as the force that arises from the radiative field of Equation (473), which we express as

$$
e F_{\mu \nu}^{\mathrm{R}} u^{\nu}=e^{2}\left(g_{\mu \nu}+u_{\mu} u_{\nu}\right)\left(\frac{2}{3} \dot{a}^{\nu}+\frac{1}{3} R_{\lambda}^{\nu} u^{\lambda}\right)+e F_{\mu \nu}^{\text {tail }} u^{\nu} .
$$

The force acting on the point particle can therefore be thought of as originating from the (smooth) radiative field, while the singular field simply contributes to the particle's inertia. After mass renormalization, Equation (478) is equivalent to the statement

$$
m a_{\mu}=e F_{\mu \nu}^{\mathrm{R}}(z) u^{\nu}
$$

where we have dropped the superfluous label "obs" on the particle's observed mass.

For the final expression of the equations of motion we follow the discussion of Section 5.1.6 and allow an external force $f_{\text {ext }}^{\mu}$ to act on the particle, and we replace, on the right-hand side of the equations, the acceleration vector by $f_{\text {ext }}^{\mu} / m$. This produces

$$
m \frac{D u^{\mu}}{d \tau}=f_{\mathrm{ext}}^{\mu}+e^{2}\left(\delta^{\mu}{ }_{\nu}+u^{\mu} u_{\nu}\right)\left(\frac{2}{3 m} \frac{D f_{\mathrm{ext}}^{\nu}}{d \tau}+\frac{1}{3} R^{\nu}{ }_{\lambda} u^{\lambda}\right)+2 e^{2} u_{\nu} \int_{-\infty}^{\tau^{-}} \nabla^{[\mu} G_{+\lambda^{\prime}}{ }^{\nu]}\left(z(\tau), z\left(\tau^{\prime}\right)\right) u^{\lambda^{\prime}} d \tau^{\prime},
$$


in which $m$ denotes the observed inertial mass of the electric charge and all tensors are evaluated at $z(\tau)$, the current position of the particle on the world line; the primed indices in the tail integral refer to the point $z\left(\tau^{\prime}\right)$, which represents a prior position. We recall that the integration must be cut short at $\tau^{\prime}=\tau^{-} \equiv \tau-0^{+}$to avoid the singular behaviour of the retarded Green's function at coincidence; this procedure was justified at the beginning of Section 5.2.3. Equation (481) was first derived (without the Ricci-tensor term) by Bryce S. DeWitt and Robert W. Brehme in 1960 [24], and then corrected by J.M. Hobbs in 1968 [29]. An alternative derivation was produced by Theodore C. Quinn and Robert M. Wald in 1997 [49]. In a subsequent publication [50], Quinn and Wald proved that the total work done by the electromagnetic self-force matches the energy radiated away by the particle.

\subsection{Motion of a point mass}

\subsubsection{Dynamics of a point mass}

In this section we consider the motion of a point particle of mass $m$ subjected to its own gravitational field. The particle moves on a world line $\gamma$ in a curved spacetime whose background metric $g_{\alpha \beta}$ is assumed to be a vacuum solution to the Einstein field equations. We shall suppose that $m$ is small, so that the perturbation $h_{\alpha \beta}$ created by the particle can also be considered to be small; it will obey a linear wave equation in the background spacetime. This linearization of the field equations will allow us to fit the problem of determining the motion of a point mass within the framework developed in Sections 5.1 and 5.2, and we shall obtain the equations of motion by following the same general line of reasoning. We shall find that $\gamma$ is not a geodesic of the background spacetime because $h_{\alpha \beta}$ acts on the particle and induces an acceleration of order $m$; the motion is geodesic in the test-mass limit only.

Our discussion in this first section is largely formal: As in Sections 5.1.1 and 5.2.1 we insert the point particle in the background spacetime and ignore the fact that the field it produces is singular on the world line. To make sense of the formal equations of motion will be our goal in the following Sections 5.3.2, 5.3.3, 5.3.4, 5.3.5, 5.3.6, and 5.3.7. The problem of determining the motion of a small mass in a background spacetime will be reconsidered in Section 5.4 from a different and more satisfying premise: There the small body will be modeled as a black hole instead of as a point particle, and the singular behaviour of the perturbation will automatically be eliminated.

Let a point particle of mass $m$ move on a world line $\gamma$ in a curved spacetime with metric $\mathrm{g}_{\alpha \beta}$. This is the total metric of the perturbed spacetime, and it depends on $m$ as well as all other relevant parameters. At a later stage of the discussion the total metric will be broken down into a "background" part $g_{\alpha \beta}$ that is independent of $m$, and a "perturbation" part $h_{\alpha \beta}$ that is proportional to $m$. The world line is described by relations $z^{\mu}(\lambda)$ in which $\lambda$ is an arbitrary parameter - this will later be identified with proper time $\tau$ in the background spacetime. In this and the following sections we will use sans-serif symbols to denote tensors that refer to the perturbed spacetime; tensors in the background spacetime will be denoted, as usual, by italic symbols.

The particle's action functional is

$$
S_{\text {particle }}=-m \int_{\gamma} \sqrt{-\mathrm{g}_{\mu \nu} \dot{z}^{\mu} \dot{z}^{\nu}} d \lambda,
$$

where $\dot{z}^{\mu}=d z^{\mu} / d \lambda$ is tangent to the world line and the metric is evaluated at $z$. We assume that the particle provides the only source of matter in the spacetime - an explanation will be provided at the end of this section - so that the Einstein field equations take the form of

$$
\mathrm{G}^{\alpha \beta}=8 \pi \mathrm{T}^{\alpha \beta},
$$

Living Reviews in Relativity

http: //www . livingreviews . org/lrr-2004-6 
where $\mathrm{G}^{\alpha \beta}$ is the Einstein tensor constructed from $\mathrm{g}_{\alpha \beta}$, and

$$
\mathrm{T}^{\alpha \beta}(x)=m \int_{\gamma} \frac{\mathrm{g}_{\mu}^{\alpha}(x, z) \mathrm{g}_{\nu}^{\beta}(x, z) \dot{z}^{\mu} \dot{z}^{\nu}}{\sqrt{-\mathrm{g}_{\mu \nu} \dot{z}^{\mu} \dot{z}^{\nu}}} \delta_{4}(x, z) d \lambda
$$

is the particle's stress-energy tensor, obtained by functional differentiation of $S_{\text {particle }}$ with respect to $\mathrm{g}_{\alpha \beta}(x)$; the parallel propagators appear naturally by expressing $\mathrm{g}_{\mu \nu}$ as $\mathrm{g}_{\mu}^{\alpha} \mathrm{g}_{\nu}^{\beta} \mathrm{g}_{\alpha \beta}$.

On a formal level the metric $\mathrm{g}_{\alpha \beta}$ is obtained by solving the Einstein field equations, and the world line is determined by solving the equations of energy-momentum conservation, which follow from the field equations. From Equations $(81,260,484)$ we obtain

$$
\nabla_{\beta} \mathrm{T}^{\alpha \beta}=m \int_{\gamma} \frac{d}{d \lambda}\left(\frac{\mathrm{g}_{\mu}^{\alpha} \dot{z}^{\mu}}{\sqrt{-\mathrm{g}_{\mu \nu} \dot{z}^{\mu} \dot{z}^{\nu}}}\right) \delta_{4}(x, z) d \lambda,
$$

and additional manipulations reduce this to

$$
\nabla_{\beta} \mathrm{T}^{\alpha \beta}=m \int_{\gamma} \frac{\mathrm{g}_{\mu}^{\alpha}}{\sqrt{-\mathrm{g}_{\mu \nu} \dot{z}^{\mu} \dot{z}^{\nu}}}\left(\frac{\mathrm{D} \dot{z}^{\mu}}{d \lambda}-\mathrm{k} \dot{z}^{\mu}\right) \delta_{4}(x, z) d \lambda,
$$

where $\mathrm{D} \dot{z}^{\mu} / d \lambda$ is the covariant acceleration and $\mathrm{k}$ is a scalar field on the world line. Energymomentum conservation therefore produces the geodesic equation

$$
\frac{\mathrm{D} \dot{z}^{\mu}}{d \lambda}=\mathrm{k} \dot{z}^{\mu},
$$

and

$$
\mathrm{k} \equiv \frac{1}{\sqrt{-\mathrm{g}_{\mu \nu} \dot{z}^{\mu} \dot{z}^{\nu}}} \frac{d}{d \lambda} \sqrt{-\mathrm{g}_{\mu \nu} \dot{z}^{\mu} \dot{z}^{\nu}}
$$

measures the failure of $\lambda$ to be an affine parameter on the geodesic $\gamma$.

At this stage we begin treating $m$ as a formal expansion parameter, and we write

$$
\mathrm{g}_{\alpha \beta}=g_{\alpha \beta}+h_{\alpha \beta}+\mathcal{O}\left(m^{2}\right),
$$

with $g_{\alpha \beta}$ denoting the $m \rightarrow 0$ limit of the total metric $\mathrm{g}_{\alpha \beta}$, and $h_{\alpha \beta}=\mathcal{O}(m)$ the first-order correction. We shall refer to $g_{\alpha \beta}$ as the "metric of the background spacetime" and to $h_{\alpha \beta}$ as the "perturbation" produced by the particle. We similarly write

$$
\mathrm{G}^{\alpha \beta}[\mathrm{g}]=G^{\alpha \beta}[g]+H^{\alpha \beta}[g ; h]+\mathcal{O}\left(m^{2}\right)
$$

for the Einstein tensor, and

$$
\mathrm{T}^{\alpha \beta}=T^{\alpha \beta}+\mathcal{O}\left(m^{2}\right)
$$

for the particle's stress-energy tensor. The leading term $T^{\alpha \beta}(x)$ describes the stress-energy tensor of a test particle of mass $m$ that moves on a world line $\gamma$ in a background spacetime with metric $g_{\alpha \beta}$. If we choose $\lambda$ to be proper time $\tau$ as measured in this spacetime, then Equation (484) implies

$$
T^{\alpha \beta}(x)=m \int_{\gamma} g_{\mu}^{\alpha}(x, z) g_{\nu}^{\beta}(x, z) u^{\mu} u^{\nu} \delta_{4}(x, z) d \tau,
$$

where $u^{\mu}(\tau)=d z^{\mu} / d \tau$ is the particle's four-velocity.

We have already stated that the particle is the only source of matter in the spacetime, and the metric $g_{\alpha \beta}$ must therefore be a solution to the vacuum field equations: $G^{\alpha \beta}[g]=0$. Equations (483, 488 , (489) then imply $H^{\alpha \beta}[g ; h]=8 \pi T^{\alpha \beta}$, in which both sides of the equation are of order $m$. To 
simplify the expression of the first-order correction to the Einstein tensor we introduce the tracereversed gravitational potentials

$$
\gamma_{\alpha \beta}=h_{\alpha \beta}-\frac{1}{2}\left(g^{\gamma \delta} h_{\gamma \delta}\right) g_{\alpha \beta}
$$

and we impose the Lorenz gauge condition

$$
\gamma_{; \beta}^{\alpha \beta}=0
$$

Here and below it is understood that indices are lowered and raised with the background metric and its inverse, respectively, and that covariant differentiation refers to a connection that is compatible with $g_{\alpha \beta}$. We then have $H^{\alpha \beta}=-\frac{1}{2}\left(\square \gamma^{\alpha \beta}+2 R_{\gamma \delta}^{\alpha{ }^{\beta}} \gamma^{\gamma \delta}\right)$, and Equation (483) reduces to

$$
\square \gamma^{\alpha \beta}+2 R_{\gamma \delta}^{\alpha \beta} \gamma^{\gamma \delta}=-16 \pi T^{\alpha \beta}
$$

where $\square=g^{\alpha \beta} \nabla_{\alpha} \nabla_{\beta}$ is the wave operator and $T^{\alpha \beta}$ is defined by Equation (490). We have here a linear wave equation for the potentials $\gamma_{\alpha \beta}$, and this equation can be placed on an equal footing with Equation (385) for the potential $\Phi$ associated with a point scalar charge, and Equation (441) for the vector potential $A^{\alpha}$ associated with a point electric charge.

The equations of motion for the point mass are obtained by substituting the expansion of Equation (487) into Equations (485) and (486). The perturbed connection is easily computed to be $\Gamma_{\beta \gamma}^{\alpha}+\frac{1}{2}\left(h_{\beta ; \gamma}^{\alpha}+h_{\gamma ; \beta}^{\alpha}-h_{\beta \gamma}{ }^{; \alpha}\right)$, and this leads to

$$
\frac{\mathrm{D} \dot{z}^{\mu}}{d \tau}=\frac{D u^{\mu}}{d \tau}+\frac{1}{2}\left(h_{\nu ; \lambda}^{\mu}+h_{\lambda ; \nu}^{\mu}-h_{\nu \lambda}^{; \mu}\right) u^{\nu} u^{\lambda}+\mathcal{O}\left(m^{2}\right)
$$

having once more selected proper time $\tau$ (as measured in the background spacetime) as the parameter on the world line. On the other hand, Equation (486) gives

$$
\mathrm{k}=-\frac{1}{2} h_{\nu \lambda ; \rho} u^{\nu} u^{\lambda} u^{\rho}-h_{\nu \lambda} u^{\nu} a^{\lambda}+\mathcal{O}\left(m^{2}\right)
$$

where $a^{\lambda}=D u^{\lambda} / d \tau$ is the particle's acceleration vector. Since it is clear that the acceleration will be of order $m$, the second term can be discarded and we obtain

$$
\frac{D u^{\mu}}{d \tau}=-\frac{1}{2}\left(h_{\nu ; \lambda}^{\mu}+h_{\lambda ; \nu}^{\mu}-h_{\nu \lambda}^{; \mu}+u^{\mu} h_{\nu \lambda ; \rho} u^{\rho}\right) u^{\nu} u^{\lambda}+\mathcal{O}\left(m^{2}\right)
$$

Keeping the error term implicit, we shall express this in the equivalent form

$$
\frac{D u^{\mu}}{d \tau}=-\frac{1}{2}\left(g^{\mu \nu}+u^{\mu} u^{\nu}\right)\left(2 h_{\nu \lambda ; \rho}-h_{\lambda \rho ; \nu}\right) u^{\lambda} u^{\rho}
$$

which emphasizes the fact that the acceleration is orthogonal to the four-velocity.

It should be clear that Equation (494) is valid only in a formal sense, because the potentials obtained from Equations (493) diverge on the world line. The nonlinearity of the Einstein field equations makes this problem even worse here than for the scalar and electromagnetic cases, because the singular behaviour of the perturbation might render meaningless a formal expansion of $\mathrm{g}_{\alpha \beta}$ in powers of $m$. Ignoring this issue for the time being (we shall return to it in Section 5.4), we will proceed as in Sections 5.1 and 5.2 and attempt, with a careful analysis of the field's singularity structure, to make sense of these equations.

To conclude this section I should explain why it is desirable to restrict our discussion to spacetimes that contain no matter except for the point particle. Suppose, in contradiction with this

Living Reviews in Relativity

http: //www . livingreviews . org/lrr-2004-6 
assumption, that the background spacetime contains a distribution of matter around which the particle is moving. (The corresponding vacuum situation has the particle moving around a black hole. Notice that we are still assuming that the particle moves in a region of spacetime in which there is no matter; the issue is whether we can allow for a distribution of matter somewhere else.) Suppose also that the matter distribution is described by a collection of matter fields $\Psi$. Then the field equations satisfied by the matter have the schematic form $E[\Psi ; g]=0$, and the metric is determined by the Einstein field equations $G[g]=8 \pi M[\Psi ; g]$, in which $M[\Psi ; g]$ stands for the matter's stress-energy tensor. We now insert the point particle in the spacetime, and recognize that this displaces the background solution $(\Psi, g)$ to a new solution $(\Psi+\delta \Psi, g+\delta g)$. The perturbations are determined by the coupled set of equations $E[\Psi+\delta \Psi ; g+\delta g]=0$ and $G[g+\delta g]=8 \pi M[\Psi+\delta \Psi ; g+\delta g]+8 \pi T[g]$. After linearization these take the form of

$$
E_{\Psi} \cdot \delta \Psi+E_{g} \cdot \delta g=0, \quad G_{g} \cdot \delta g=8 \pi\left(M_{\Psi} \cdot \delta \Psi+M_{g} \cdot \delta g+T\right),
$$

where $E_{\Psi}, E_{g}, M_{\Phi}$, and $M_{g}$ are suitable differential operators acting on the perturbations. This is a coupled set of partial differential equations for the perturbations $\delta \Psi$ and $\delta g$. These equations are linear, but they are much more difficult to deal with than the single equation for $\delta g$ that was obtained in the vacuum case. And although it is still possible to solve the coupled set of equations via a Green's function technique, the degree of difficulty is such that we will not attempt this here. We shall, therefore, continue to restrict our attention to the case of a point particle moving in a vacuum (globally Ricci-flat) background spacetime.

\subsubsection{Retarded potentials near the world line}

The retarded solution to Equation (493) is $\gamma^{\alpha \beta}(x)=4 \int G_{+\gamma^{\prime} \delta^{\prime}}^{\alpha \beta}\left(x, x^{\prime}\right) T^{\gamma^{\prime} \delta^{\prime}}\left(x^{\prime}\right) \sqrt{-g^{\prime}} d^{4} x^{\prime}$, where $G_{+\gamma^{\prime} \delta^{\prime}}^{\alpha \beta}\left(x, x^{\prime}\right)$ is the retarded Green's function introduced in Section 4.5. After substitution of the stress-energy tensor of Equation (490) we obtain

$$
\gamma^{\alpha \beta}(x)=4 m \int_{\gamma} G_{+\mu \nu}^{\alpha \beta}(x, z) u^{\mu} u^{\nu} d \tau
$$

in which $z^{\mu}(\tau)$ gives the description of the world line $\gamma$ and $u^{\mu}=d z^{\mu} / d \tau$. Because the retarded Green's function is defined globally in the entire background spacetime, Equation (495) describes the gravitational perturbation created by the particle at any point $x$ in that spacetime.

For a more concrete expression we must take $x$ to be in a neighbourhood of the world line. The following manipulations follow closely those performed in Section 5.1.2 for the case of a scalar charge, and in Section 5.2.2 for the case of an electric charge. Because these manipulations are by now familiar, it will be sufficient here to present only the main steps. There are two important simplifications that occur in the case of a massive particle. First, for the purposes of computing $\gamma^{\alpha \beta}(x)$ to first order in $m$, it is sufficient to take the world line to be a geodesic of the background spacetime: The deviations from geodesic motion that we are in the process of calculating are themselves of order $m$ and would affect $\gamma^{\alpha \beta}(x)$ at order $m^{2}$ only. We shall therefore be allowed to set

$$
a^{\mu}=0=\dot{a}^{\mu}
$$

in our computations. Second, because we take $g_{\alpha \beta}$ to be a solution to the vacuum field equations, we are also allowed to set

$$
R_{\mu \nu}(z)=0
$$

in our computations.

With the understanding that $x$ is close to the world line (refer back to Figure 9), we substitute the Hadamard construction of Equation (352) into Equation (495) and integrate over the portion 
of $\gamma$ that is contained in $\mathcal{N}(x)$. The result is

$$
\gamma^{\alpha \beta}(x)=\frac{4 m}{r} U_{\gamma^{\prime} \delta^{\prime}}^{\alpha \beta}\left(x, x^{\prime}\right) u^{\gamma^{\prime}} u^{\delta^{\prime}}+4 m \int_{\tau_{<}}^{u} V_{\mu \nu}^{\alpha \beta}(x, z) u^{\mu} u^{\nu} d \tau+4 m \int_{-\infty}^{\tau_{<}} G_{+\mu \nu}^{\alpha \beta}(x, z) u^{\mu} u^{\nu} d \tau,
$$

in which primed indices refer to the retarded point $x^{\prime} \equiv z(u)$ associated with $x, r \equiv \sigma_{\alpha^{\prime}} u^{\alpha^{\prime}}$ is the retarded distance from $x^{\prime}$ to $x$, and $\tau_{<}$is the proper time at which $\gamma$ enters $\mathcal{N}(x)$ from the past.

In the following Sections 5.3.3, 5.3.4, 5.3.5, 5.3.6, and 5.3.7, we shall refer to $\gamma_{\alpha \beta}(x)$ as the gravitational potentials at $x$ produced by a particle of mass $m$ moving on the world line $\gamma$, and to $\gamma_{\alpha \beta ; \gamma}(x)$ as the gravitational field at $x$. To compute this is our next task.

\subsubsection{Gravitational field in retarded coordinates}

Keeping in mind that $x^{\prime}$ and $x$ are related by $\sigma\left(x, x^{\prime}\right)=0$, a straightforward computation reveals that the covariant derivatives of the gravitational potentials are given by

$$
\begin{aligned}
\gamma_{\alpha \beta ; \gamma}(x)= & -\frac{4 m}{r^{2}} U_{\alpha \beta \alpha^{\prime} \beta^{\prime}} u^{\alpha^{\prime}} u^{\beta^{\prime}} \partial_{\gamma} r+\frac{4 m}{r} U_{\alpha \beta \alpha^{\prime} \beta^{\prime} ; \gamma} u^{\alpha^{\prime}} u^{\beta^{\prime}}+\frac{4 m}{r} U_{\alpha \beta \alpha^{\prime} \beta^{\prime} ; \gamma^{\prime}} u^{\alpha^{\prime}} u^{\beta^{\prime}} u^{\gamma^{\prime}} \partial_{\gamma} u \\
& +4 m V_{\alpha \beta \alpha^{\prime} \beta^{\prime}} u^{\alpha^{\prime}} u^{\beta^{\prime}} \partial_{\gamma} u+\gamma_{\alpha \beta \gamma}^{\text {tail }}(x)
\end{aligned}
$$

where the "tail integral" is defined by

$$
\begin{aligned}
\gamma_{\alpha \beta \gamma}^{\text {tail }}(x) & =4 m \int_{\tau_{<}}^{u} \nabla_{\gamma} V_{\alpha \beta \mu \nu}(x, z) u^{\mu} u^{\nu} d \tau+4 m \int_{-\infty}^{\tau_{<}} \nabla_{\gamma} G_{+\alpha \beta \mu \nu}(x, z) u^{\mu} u^{\nu} d \tau \\
& =4 m \int_{-\infty}^{u^{-}} \nabla_{\gamma} G_{+\alpha \beta \mu \nu}(x, z) u^{\mu} u^{\nu} d \tau .
\end{aligned}
$$

The second form of the definition, in which the integration is cut short at $\tau=u^{-} \equiv u-0^{+}$to avoid the singular behaviour of the retarded Green's function at $\sigma=0$, is equivalent to the first form.

We wish to express $\gamma_{\alpha \beta ; \gamma}(x)$ in the retarded coordinates of Section 3.3, as an expansion in powers of $r$. For this purpose we decompose the field in the tetrad $\left(e_{0}^{\alpha}, e_{a}^{\alpha}\right)$ that is obtained by parallel transport of $\left(u^{\alpha^{\prime}}, e_{a}^{\alpha^{\prime}}\right)$ on the null geodesic that links $x$ to $x^{\prime}$; this construction is detailed in Section 3.3. Note that throughout this section we set $\omega_{a b}=0$, where $\omega_{a b}$ is the rotation tensor defined by Equation (138): The tetrad vectors $e_{a}^{\alpha^{\prime}}$ are taken to be parallel transported on $\gamma$. We recall from Equation (141) that the parallel propagator can be expressed as $g_{\alpha}^{\alpha^{\prime}}=u^{\alpha^{\prime}} e_{\alpha}^{0}+e_{a}^{\alpha^{\prime}} e_{\alpha}^{a}$. The expansion relies on Equation (166) for $\partial_{\gamma} u$ and Equation (168) for $\partial_{\gamma} r$, both specialized to the case of geodesic motio, $a_{a}=0$. We shall also need

$$
U_{\alpha \beta \alpha^{\prime} \beta^{\prime}} u^{\alpha^{\prime}} u^{\beta^{\prime}}=g_{(\alpha}^{\alpha^{\prime}} g_{\beta)}^{\beta^{\prime}}\left[u_{\alpha^{\prime}} u_{\beta^{\prime}}+\mathcal{O}\left(r^{3}\right)\right],
$$

which follows from Equation (358),

$$
\begin{aligned}
U_{\alpha \beta \alpha^{\prime} \beta^{\prime} ; \gamma} u^{\alpha^{\prime}} u^{\beta^{\prime}} & =g_{(\alpha}^{\alpha^{\prime}} g_{\beta)}^{\beta^{\prime}} g_{\gamma}^{\gamma^{\prime}}\left[-r\left(R_{\alpha^{\prime} 0 \gamma^{\prime} 0}+R_{\alpha^{\prime} 0 \gamma^{\prime} d} \Omega^{d}\right) u_{\beta^{\prime}}+\mathcal{O}\left(r^{2}\right)\right], \\
U_{\alpha \beta \alpha^{\prime} \beta^{\prime} ; \gamma^{\prime}} u^{\alpha^{\prime}} u^{\beta^{\prime}} u^{\gamma^{\prime}} & =g_{(\alpha}^{\alpha^{\prime}} g_{\beta)}^{\beta^{\prime}}\left[r R_{\alpha^{\prime} 0 d 0} \Omega^{d} u_{\beta^{\prime}}+\mathcal{O}\left(r^{2}\right)\right],
\end{aligned}
$$

which follow from Equations (359) and (360), respectively, as well as the relation $\sigma^{\alpha^{\prime}}=-r\left(u^{\alpha^{\prime}}+\right.$ $\left.\Omega^{a} e_{a}^{\alpha^{\prime}}\right)$ first encountered in Equation (144). And finally, we shall need

$$
V_{\alpha \beta \alpha^{\prime} \beta^{\prime}} u^{\alpha^{\prime}} u^{\beta^{\prime}}=g_{(\alpha}^{\alpha^{\prime}} g_{\beta)}^{\beta^{\prime}}\left[R_{\alpha^{\prime} 0 \beta^{\prime} 0}+\mathcal{O}(r)\right]
$$

Living Reviews in Relativity

http: //www . livingreviews . org/lrr-2004-6 
which follows from Equation (362).

Making these substitutions in Equation (484) and projecting against various members of the tetrad gives

$$
\begin{aligned}
\gamma_{000}\left(u, r, \Omega^{a}\right) & \equiv \gamma_{\alpha \beta ; \gamma}(x) e_{0}^{\alpha}(x) e_{0}^{\beta}(x) e_{0}^{\gamma}(x)=2 m R_{a 0 b 0} \Omega^{a} \Omega^{b}+\gamma_{000}^{\text {tail }}+\mathcal{O}(r) \\
\gamma_{0 b 0}\left(u, r, \Omega^{a}\right) & \equiv \gamma_{\alpha \beta ; \gamma}(x) e_{0}^{\alpha}(x) e_{b}^{\beta}(x) e_{0}^{\gamma}(x)=-4 m R_{b 0 c 0} \Omega^{c}+\gamma_{0 b 0}^{\text {tail }}+\mathcal{O}(r) \\
\gamma_{a b 0}\left(u, r, \Omega^{a}\right) & \equiv \gamma_{\alpha \beta ; \gamma}(x) e_{a}^{\alpha}(x) e_{b}^{\beta}(x) e_{0}^{\gamma}(x)=4 m R_{a 0 b 0}+\gamma_{a b 0}^{\text {tail }}+\mathcal{O}(r) \\
\gamma_{00 c}\left(u, r, \Omega^{a}\right) & \equiv \gamma_{\alpha \beta ; \gamma}(x) e_{0}^{\alpha}(x) e_{0}^{\beta}(x) e_{c}^{\gamma}(x) \\
& =-4 m\left[\left(\frac{1}{r^{2}}+\frac{1}{3} R_{a 0 b 0} \Omega^{a} \Omega^{b}\right) \Omega_{c}+\frac{1}{6} R_{c 0 b 0} \Omega^{b}-\frac{1}{6} R_{c a 0 b} \Omega^{a} \Omega^{b}\right]+\gamma_{00 c}^{\text {tail }}+\mathcal{O}(r), \\
\gamma_{0 b c}\left(u, r, \Omega^{a}\right) & \equiv \gamma_{\alpha \beta ; \gamma}(x) e_{0}^{\alpha}(x) e_{b}^{\beta}(x) e_{c}^{\gamma}(x) \\
& =2 m\left(R_{b 0 c 0}+R_{b 0 c d} \Omega^{d}+R_{b 0 d 0} \Omega^{d} \Omega_{c}\right)+\gamma_{0 b c}^{\text {tail }}+\mathcal{O}(r) \\
\gamma_{a b c}\left(u, r, \Omega^{a}\right) & \equiv \gamma_{\alpha \beta ; \gamma}(x) e_{a}^{\alpha}(x) e_{b}^{\beta}(x) e_{c}^{\gamma}(x)=-4 m R_{a 0 b 0} \Omega_{c}+\gamma_{a b c}^{\text {tail }}+\mathcal{O}(r),
\end{aligned}
$$

where, for example, $R_{a 0 b 0}(u) \equiv R_{\alpha^{\prime} \gamma^{\prime} \beta^{\prime} \delta^{\prime}} e_{a}^{\alpha^{\prime}} u^{\gamma^{\prime}} e_{b}^{\beta^{\prime}} u^{\delta^{\prime}}$ are frame components of the Riemann tensor evaluated at $x^{\prime} \equiv z(u)$. We have also introduced the frame components of the tail part of the gravitational field, which are obtained from Equation (500) evaluated at $x^{\prime}$ instead of $x$; for example, $\gamma_{000}^{\text {tail }}=u^{\alpha^{\prime}} u^{\beta^{\prime}} u^{\gamma^{\prime}} \gamma_{\alpha^{\prime} \beta^{\prime} \gamma^{\prime}}^{\text {tail }}\left(x^{\prime}\right)$. We may note here that while $\gamma_{00 c}$ is the only component of the gravitational field that diverges when $r \rightarrow 0$, the other components are nevertheless singular because of their dependence on the unit vector $\Omega^{a}$; the only exception is $\gamma_{a b 0}$, which is smooth.

\subsubsection{Gravitational field in Fermi normal coordinates}

The translation of the results contained in Equations $(505,506,507,508,509,510)$ into the Fermi normal coordinates of Section 3.2 proceeds as in Sections 5.1.4 and 5.2.4, but is simplified by the fact that here the world line can be taken to be a geodesic. We may thus set $a_{a}=\dot{a}_{0}=\dot{a}_{a}=0$ in Equations (224) and (225) that relate the tetrad $\left(\bar{e}_{0}^{\alpha}, \bar{e}_{a}^{\alpha}\right)$ to $\left(e_{0}^{\alpha}, e_{a}^{\alpha}\right)$, as well as in Equations (221, $222,223)$ that relate the Fermi normal coordinates $\left(t, s, \omega^{a}\right)$ to the retarded coordinates. We recall that the Fermi normal coordinates refer to a point $\bar{x} \equiv z(t)$ on the world line that is linked to $x$ by a spacelike geodesic that intersects $\gamma$ orthogonally.

The translated results are

$$
\begin{aligned}
\bar{\gamma}_{000}\left(t, s, \omega^{a}\right) & \equiv \gamma_{\alpha \beta ; \gamma}(x) \bar{e}_{0}^{\alpha}(x) \bar{e}_{0}^{\beta}(x) \bar{e}_{0}^{\gamma}(x)=\bar{\gamma}_{000}^{\text {tail }}+\mathcal{O}(s), \\
\bar{\gamma}_{0 b 0}\left(t, s, \omega^{a}\right) & \equiv \gamma_{\alpha \beta ; \gamma}(x) \bar{e}_{0}^{\alpha}(x) \bar{e}_{b}^{\beta}(x) \bar{e}_{0}^{\gamma}(x)=-4 m R_{b 0 c 0} \omega^{c}+\bar{\gamma}_{0 b 0}^{\mathrm{tail}}+\mathcal{O}(s), \\
\bar{\gamma}_{a b 0}\left(t, s, \omega^{a}\right) & \equiv \gamma_{\alpha \beta ; \gamma}(x) \bar{e}_{a}^{\alpha}(x) \bar{e}_{b}^{\beta}(x) \bar{e}_{0}^{\gamma}(x)=4 m R_{a 0 b 0}+\bar{\gamma}_{a b 0}^{\text {tail }}+\mathcal{O}(s), \\
\bar{\gamma}_{00 c}\left(t, s, \omega^{a}\right) & \equiv \gamma_{\alpha \beta ; \gamma}(x) \bar{e}_{0}^{\alpha}(x) \bar{e}_{0}^{\beta}(x) \bar{e}_{c}^{\gamma}(x) \\
& =-4 m\left[\left(\frac{1}{s^{2}}-\frac{1}{6} R_{a 0 b 0} \omega^{a} \omega^{b}\right) \omega_{c}+\frac{1}{3} R_{c 0 b 0} \omega^{b}\right]+\bar{\gamma}_{00 c}^{\text {tail }}+\mathcal{O}(s), \\
\bar{\gamma}_{0 b c}\left(t, s, \omega^{a}\right) & \equiv \gamma_{\alpha \beta ; \gamma}(x) \bar{e}_{0}^{\alpha}(x) \bar{e}_{b}^{\beta}(x) \bar{e}_{c}^{\gamma}(x)=2 m\left(R_{b 0 c 0}+R_{b 0 c d} \omega^{d}\right)+\bar{\gamma}_{0 b c}^{\text {tail }}+\mathcal{O}(s), \\
\bar{\gamma}_{a b c}\left(t, s, \omega^{a}\right) & \equiv \gamma_{\alpha \beta ; \gamma}(x) \bar{e}_{a}^{\alpha}(x) \bar{e}_{b}^{\beta}(x) \bar{e}_{c}^{\gamma}(x)=-4 m R_{a 0 b 0} \omega_{c}+\bar{\gamma}_{a b c}^{\text {tail }}+\mathcal{O}(s),
\end{aligned}
$$

where all frame components are now evaluated at $\bar{x}$ instead of $x^{\prime}$.

It is then a simple matter to average these results over a two-surface of constant $t$ and $s$. Using 
the area element of Equation (404) and definitions analogous to those of Equation (405), we obtain

$$
\begin{aligned}
\left\langle\bar{\gamma}_{000}\right\rangle & =\bar{\gamma}_{000}^{\text {tail }}+\mathcal{O}(s), \\
\left\langle\bar{\gamma}_{0 b 0}\right\rangle & =\bar{\gamma}_{0 b 0}^{\text {tail }}+\mathcal{O}(s), \\
\left\langle\bar{\gamma}_{a b 0}\right\rangle & =4 m R_{a 0 b 0}+\bar{\gamma}_{a b 0}^{\text {tail }}+\mathcal{O}(s), \\
\left\langle\bar{\gamma}_{00 c}\right\rangle & =\bar{\gamma}_{00 c}^{\text {tail }}+\mathcal{O}(s), \\
\left\langle\bar{\gamma}_{0 b c}\right\rangle & =2 m R_{b 0 c 0}+\bar{\gamma}_{0 b c}^{\text {tail }}+\mathcal{O}(s), \\
\left\langle\bar{\gamma}_{a b c}\right\rangle & =\bar{\gamma}_{a b c}^{\text {tail }}+\mathcal{O}(s) .
\end{aligned}
$$

The averaged gravitational field is smooth in the limit $s \rightarrow 0$, in which the tetrad $\left(\bar{e}_{0}^{\alpha}, \bar{e}_{a}^{\alpha}\right)$ coincides with $\left(u^{\bar{\alpha}}, e_{a}^{\bar{\alpha}}\right)$. Reconstructing the field at $\bar{x}$ from its frame components gives

$$
\left\langle\gamma_{\bar{\alpha} \bar{\beta} ; \bar{\gamma}}\right\rangle=-4 m\left(u_{(\bar{\alpha}} R_{\bar{\beta}) \bar{\delta} \bar{\gamma} \bar{\epsilon}}+R_{\bar{\alpha} \bar{\delta} \bar{\beta} \bar{\epsilon}} u_{\bar{\gamma}}\right) u^{\bar{\delta}} u^{\bar{\epsilon}}+\gamma_{\bar{\alpha} \bar{\beta} \bar{\gamma}}^{\text {tail }}
$$

where the tail term can be copied from Equation (500),

$$
\gamma_{\bar{\alpha} \bar{\beta} \bar{\gamma}}^{\mathrm{tail}}(\bar{x})=4 m \int_{-\infty}^{t^{-}} \nabla_{\bar{\gamma}} G_{+\bar{\alpha} \bar{\beta} \mu \nu}(\bar{x}, z) u^{\mu} u^{\nu} d \tau .
$$

The tensors that appear in Equation (523) all refer to the simultaneous point $\bar{x} \equiv z(t)$, which can now be treated as an arbitrary point on the world line $\gamma$.

\subsubsection{Singular and radiative fields}

The singular gravitational potentials

$$
\gamma_{\mathrm{S}}^{\alpha \beta}(x)=4 m \int_{\gamma} G_{\mathrm{S} \mu \nu}^{\alpha \beta}(x, z) u^{\mu} u^{\nu} d \tau
$$

are solutions to the wave equation of Equation (493); the singular Green's function was introduced in Section 4.5.4. We will see that the singular field $\gamma_{\alpha \beta ; \gamma}^{\mathrm{S}}$ reproduces the singular behaviour of the retarded solution near the world line, and that the difference, $\gamma_{\alpha \beta ; \gamma}^{\mathrm{R}}=\gamma_{\alpha \beta ; \gamma}-\gamma_{\alpha \beta ; \gamma}^{\mathrm{S}}$, is smooth on the world line.

To evaluate the integral of Equation (525) we take $x$ to be close to the world line (see Figure 9), and we invoke Equation (373) as well as the Hadamard construction of Equation (379). This gives

$$
\gamma_{\mathrm{S}}^{\alpha \beta}(x)=\frac{2 m}{r} U_{\gamma^{\prime} \delta^{\prime}}^{\alpha \beta} u^{\gamma^{\prime}} u^{\delta^{\prime}}+\frac{2 m}{r_{\mathrm{adv}}} U_{\gamma^{\prime \prime} \delta^{\prime \prime}}^{\alpha \beta} u^{\gamma^{\prime \prime}} u^{\delta^{\prime \prime}}-2 m \int_{u}^{v} V_{\mu \nu}^{\alpha \beta}(x, z) u^{\mu} u^{\nu} d \tau,
$$

where primed indices refer to the retarded point $x^{\prime} \equiv z(u)$, double-primed indices refer to the advanced point $x^{\prime \prime} \equiv z(v)$, and where $r_{\mathrm{adv}} \equiv-\sigma_{\alpha^{\prime \prime}} u^{\alpha^{\prime \prime}}$ is the advanced distance between $x$ and the world line.

Differentiation of Equation (526) yields

$$
\begin{aligned}
\gamma_{\alpha \beta ; \gamma}^{\mathrm{S}}(x)= & -\frac{2 m}{r^{2}} U_{\alpha \beta \alpha^{\prime} \beta^{\prime}} u^{\alpha^{\prime}} u^{\beta^{\prime}} \partial_{\gamma} r-\frac{2 m}{r_{\mathrm{adv}}{ }^{2}} U_{\alpha \beta \alpha^{\prime \prime} \beta^{\prime \prime}} u^{\alpha^{\prime \prime}} u^{\beta^{\prime \prime}} \partial_{\gamma} r_{\mathrm{adv}}+\frac{2 m}{r} U_{\alpha \beta \alpha^{\prime} \beta^{\prime} ; \gamma} u^{\alpha^{\prime}} u^{\beta^{\prime}} \\
& +\frac{2 m}{r} U_{\alpha \beta \alpha^{\prime} \beta^{\prime} ; \gamma^{\prime}} u^{\alpha^{\prime}} u^{\beta^{\prime}} u^{\gamma^{\prime}} \partial_{\gamma} u+\frac{2 m}{r_{\mathrm{adv}}} U_{\alpha \beta \alpha^{\prime \prime} \beta^{\prime \prime} ; \gamma} u^{\alpha^{\prime \prime}} u^{\beta^{\prime \prime}}+\frac{2 m}{r_{\mathrm{adv}}} U_{\alpha \beta \alpha^{\prime \prime} \beta^{\prime \prime} ; \gamma^{\prime \prime}} u^{\alpha^{\prime \prime}} u^{\beta^{\prime \prime}} u^{\gamma^{\prime \prime}} \partial_{\gamma} v \\
& +2 m V_{\alpha \beta \alpha^{\prime} \beta^{\prime}} u^{\alpha^{\prime}} u^{\beta^{\prime}} \partial_{\gamma} u-2 m V_{\alpha \beta \alpha^{\prime \prime} \beta^{\prime \prime}} u^{\alpha^{\prime \prime}} u^{\beta^{\prime \prime}} \partial_{\gamma} v-2 m \int_{u}^{v} \nabla_{\gamma} V_{\alpha \beta \mu \nu}(x, z) u^{\mu} u^{\nu} d \tau,(527)
\end{aligned}
$$

Living Reviews in Relativity

http: //www . livingreviews . org/lrr-2004-6 
and we would like to express this as an expansion in powers of $r$. For this we will rely on results already established in Section 5.3.3, as well as additional expansions that will involve the advanced point $x^{\prime \prime}$. We recall that a relation between retarded and advanced times was worked out in Equation (229), that an expression for the advanced distance was displayed in Equation (230), and that Equations (231) and (232) give expansions for $\partial_{\gamma} v$ and $\partial_{\gamma} r_{\text {adv }}$, respectively; these results can be simplified by setting $a_{a}=\dot{a}_{0}=\dot{a}_{a}=0$, which is appropriate in this computation.

To derive an expansion for $U_{\alpha \beta \alpha^{\prime \prime} \beta^{\prime \prime}} u^{\alpha^{\prime \prime}} u^{\beta^{\prime \prime}}$ we follow the general method of Section 3.4.4 and introduce the functions $U_{\alpha \beta}(\tau) \equiv U_{\alpha \beta \mu \nu}(x, z) u^{\mu} u^{\nu}$. We have that

$$
U_{\alpha \beta \alpha^{\prime \prime} \beta^{\prime \prime}} u^{\alpha^{\prime \prime}} u^{\beta^{\prime \prime}} \equiv U_{\alpha \beta}(v)=U_{\alpha \beta}(u)+\dot{U}_{\alpha \beta}(u) \Delta^{\prime}+\frac{1}{2} \ddot{U}_{\alpha \beta}(u) \Delta^{\prime 2}+O\left(\Delta^{\prime 3}\right),
$$

where overdots indicate differentiation with respect to $\tau$ and $\Delta^{\prime} \equiv v-u$. The leading term $U_{\alpha \beta}(u) \equiv U_{\alpha \beta \alpha^{\prime} \beta^{\prime}} u^{\alpha^{\prime}} u^{\beta^{\prime}}$ was worked out in Equation (501), and the derivatives of $U_{\alpha \beta}(\tau)$ are given by

$$
\dot{U}_{\alpha \beta}(u)=U_{\alpha \beta \alpha^{\prime} \beta^{\prime} ; \gamma^{\prime}} u^{\alpha^{\prime}} u^{\beta^{\prime}} u^{\gamma^{\prime}}=g_{(\alpha}^{\alpha^{\prime}} g_{\beta)}^{\beta^{\prime}}\left[r R_{\alpha^{\prime} 0 d 0} \Omega^{d} u_{\beta^{\prime}}+\mathcal{O}\left(r^{2}\right)\right]
$$

and

$$
\ddot{U}_{\alpha \beta}(u)=U_{\alpha \beta \alpha^{\prime} \beta^{\prime} ; \gamma^{\prime} \delta^{\prime}} u^{\alpha^{\prime}} u^{\beta^{\prime}} u^{\gamma^{\prime}} u^{\delta^{\prime}}=\mathcal{O}(r),
$$

according to Equations (503) and (360). Combining these results together with Equation (229) for $\Delta^{\prime}$ gives

$$
U_{\alpha \beta \alpha^{\prime \prime} \beta^{\prime \prime}} u^{\alpha^{\prime \prime}} u^{\beta^{\prime \prime}}=g_{(\alpha}^{\alpha^{\prime}} g_{\beta)}^{\beta^{\prime}}\left[u_{\alpha^{\prime}} u_{\beta^{\prime}}+2 r^{2} R_{\alpha^{\prime} 0 d 0} \Omega^{d} u_{\beta^{\prime}}+\mathcal{O}\left(r^{3}\right)\right],
$$

which should be compared with Equation (501). It should be emphasized that in Equation (528) and all equations below, all frame components are evaluated at the retarded point $x^{\prime}$, and not at the advanced point. The preceding computation gives us also an expansion for

$$
U_{\alpha \beta \alpha^{\prime \prime} \beta^{\prime \prime} ; \gamma^{\prime \prime}} u^{\alpha^{\prime}} u^{\beta^{\prime \prime}} u^{\gamma^{\prime \prime}}=\dot{U}_{\alpha \beta}(u)+\ddot{U}_{\alpha \beta}(u) \Delta^{\prime}+\mathcal{O}\left(\Delta^{\prime 2}\right),
$$

which becomes

$$
U_{\alpha \beta \alpha^{\prime \prime} \beta^{\prime \prime} ; \gamma^{\prime \prime}} u^{\alpha^{\prime \prime}} u^{\beta^{\prime \prime}} u^{\gamma^{\prime \prime}}=g_{(\alpha}^{\alpha^{\prime}} g_{\beta)}^{\beta^{\prime}}\left[r R_{\alpha^{\prime} 0 d 0} \Omega^{d} u_{\beta^{\prime}}+\mathcal{O}\left(r^{2}\right)\right]
$$

and which is identical to Equation (503).

We proceed similarly to obtain an expansion for $U_{\alpha \beta \alpha^{\prime \prime} \beta^{\prime \prime} ; \gamma} u^{\alpha^{\prime \prime}} u^{\beta^{\prime \prime}}$. Here we introduce the functions $U_{\alpha \beta \gamma}(\tau) \equiv U_{\alpha \beta \mu \nu ; \gamma} u^{\mu} u^{\nu}$ and express $U_{\alpha \beta \alpha^{\prime \prime} \beta^{\prime \prime} ; \gamma} u^{\alpha^{\prime \prime}} u^{\beta^{\prime \prime}}$ as $U_{\alpha \beta \gamma}(v)=U_{\alpha \beta \gamma}(u)+\dot{U}_{\alpha \beta \gamma}(u) \Delta^{\prime}+$ $\mathcal{O}\left(\Delta^{\prime 2}\right)$. The leading term $U_{\alpha \beta \gamma}(u) \equiv U_{\alpha \beta \alpha^{\prime} \beta^{\prime} ; \gamma} u^{\alpha^{\prime}} u^{\beta^{\prime}}$ was computed in Equation (502), and

$$
\dot{U}_{\alpha \beta \gamma}(u)=U_{\alpha \beta \alpha^{\prime} \beta^{\prime} ; \gamma \gamma^{\prime}} u^{\alpha^{\prime}} u^{\beta^{\prime}} u^{\gamma^{\prime}}=g_{(\alpha}^{\alpha^{\prime}} g_{\beta)}^{\beta^{\prime}} g_{\gamma}^{\gamma^{\prime}}\left[R_{\alpha^{\prime} 0 \gamma^{\prime} 0} u_{\beta^{\prime}}+\mathcal{O}(r)\right]
$$

follows from Equation (359). Combining these results together with Equation (229) for $\Delta^{\prime}$ gives

$$
U_{\alpha \beta \alpha^{\prime \prime} \beta^{\prime \prime} ; \gamma} u^{\alpha^{\prime \prime}} u^{\beta^{\prime \prime}}=g_{(\alpha}^{\alpha^{\prime}} g_{\beta)}^{\beta^{\prime}} g_{\gamma}^{\gamma^{\prime}}\left[r\left(R_{\alpha^{\prime} 0 \gamma^{\prime} 0}-R_{\alpha^{\prime} 0 \gamma^{\prime} d} \Omega^{d}\right) u_{\beta^{\prime}}+\mathcal{O}\left(r^{2}\right)\right]
$$

and this should be compared with Equation (502). The last expansion we shall need is

$$
V_{\alpha \beta \alpha^{\prime \prime} \beta^{\prime \prime}} u^{\alpha^{\prime \prime}} u^{\beta^{\prime \prime}}=g_{(\alpha}^{\alpha^{\prime}} g_{\beta)}^{\beta^{\prime}}\left[R_{\alpha^{\prime} 0 \beta^{\prime} 0}+\mathcal{O}(r)\right],
$$

which is identical to Equation (504). 
We obtain the frame components of the singular gravitational field by substituting these expansions into Equation (527) and projecting against the tetrad $\left(e_{0}^{\alpha}, e_{a}^{\alpha}\right)$. After some algebra we arrive at

$$
\begin{aligned}
\gamma_{000}^{\mathrm{S}}\left(u, r, \Omega^{a}\right) & \equiv \gamma_{\alpha \beta ; \gamma}^{\mathrm{S}}(x) e_{0}^{\alpha}(x) e_{0}^{\beta}(x) e_{0}^{\gamma}(x)=2 m R_{a 0 b 0} \Omega^{a} \Omega^{b}+\mathcal{O}(r), \\
\gamma_{0 b 0}^{\mathrm{S}}\left(u, r, \Omega^{a}\right) & \equiv \gamma_{\alpha \beta ; \gamma}^{\mathrm{S}}(x) e_{0}^{\alpha}(x) e_{b}^{\beta}(x) e_{0}^{\gamma}(x)=-4 m R_{b 0 c 0} \Omega^{c}+\mathcal{O}(r), \\
\gamma_{a b 0}^{\mathrm{S}}\left(u, r, \Omega^{a}\right) & \equiv \gamma_{\alpha \beta ; \gamma}^{\mathrm{S}}(x) e_{a}^{\alpha}(x) e_{b}^{\beta}(x) e_{0}^{\gamma}(x)=\mathcal{O}(r), \\
\gamma_{00 c}^{\mathrm{S}}\left(u, r, \Omega^{a}\right) & \equiv \gamma_{\alpha \beta ; \gamma}^{\mathrm{S}}(x) e_{0}^{\alpha}(x) e_{0}^{\beta}(x) e_{c}^{\gamma}(x) \\
& =-4 m\left[\left(\frac{1}{r^{2}}+\frac{1}{3} R_{a 0 b 0} \Omega^{a} \Omega^{b}\right) \Omega_{c}+\frac{1}{6} R_{c 0 b 0} \Omega^{b}-\frac{1}{6} R_{c a 0 b} \Omega^{a} \Omega^{b}\right]+\mathcal{O}(r), \\
\gamma_{0 b c}^{\mathrm{S}}\left(u, r, \Omega^{a}\right) & \equiv \gamma_{\alpha \beta ; \gamma}^{\mathrm{S}}(x) e_{0}^{\alpha}(x) e_{b}^{\beta}(x) e_{c}^{\gamma}(x)=2 m\left(R_{b 0 c d} \Omega^{d}+R_{b 0 d 0} \Omega^{d} \Omega_{c}\right)+\mathcal{O}(r), \\
\gamma_{a b c}^{\mathrm{S}}\left(u, r, \Omega^{a}\right) & \equiv \gamma_{\alpha \beta ; \gamma}^{\mathrm{S}}(x) e_{a}^{\alpha}(x) e_{b}^{\beta}(x) e_{c}^{\gamma}(x)=-4 m R_{a 0 b 0} \Omega_{c}+\mathcal{O}(r),
\end{aligned}
$$

in which all frame components are evaluated at the retarded point $x^{\prime}$. Comparison of these expressions with Equations $(505,506,507,508,509,510)$ reveals identical singularity structures for the retarded and singular gravitational fields.

The difference between the retarded field of Equations $(505,506,507,508,509,510)$ and the singular field of Equations $(532,533,534,535,536,537)$ defines the radiative gravitational field $\gamma_{\alpha \beta ; \gamma}^{\mathrm{R}}$. Its tetrad components are

$$
\begin{aligned}
\gamma_{000}^{\mathrm{R}} & =\gamma_{000}^{\text {tail }}+\mathcal{O}(r), \\
\gamma_{0 b 0}^{\mathrm{R}} & =\gamma_{0 b 0}^{\text {tail }}+\mathcal{O}(r), \\
\gamma_{a b 0}^{\mathrm{R}} & =4 m R_{a 0 b 0}+\gamma_{a b 0}^{\text {tail }}+\mathcal{O}(r), \\
\gamma_{00 c}^{\mathrm{R}} & =\gamma_{00 c}^{\text {tail }}+\mathcal{O}(r), \\
\gamma_{0 b c}^{\mathrm{R}} & =2 m R_{b 0 c 0}+\gamma_{0 b c}^{\text {tail }}+\mathcal{O}(r), \\
\gamma_{a b c}^{\mathrm{R}} & =\gamma_{a b c}^{\text {tail }}+\mathcal{O}(r),
\end{aligned}
$$

and we see that $\gamma_{\alpha \beta ; \gamma}^{\mathrm{R}}$ is smooth in the limit $r \rightarrow 0$. We may therefore evaluate the radiative field directly at $x=x^{\prime}$, where the tetrad $\left(e_{0}^{\alpha}, e_{a}^{\alpha}\right)$ coincides with $\left(u^{\alpha^{\prime}}, e_{a}^{\alpha^{\prime}}\right)$. After reconstructing the field at $x^{\prime}$ from its frame components, we obtain

$$
\gamma_{\alpha^{\prime} \beta^{\prime} ; \gamma^{\prime}}^{\mathrm{R}}\left(x^{\prime}\right)=-4 m\left(u_{\left(\alpha^{\prime}\right.} R_{\left.\beta^{\prime}\right) \delta^{\prime} \gamma^{\prime} \epsilon^{\prime}}+R_{\alpha^{\prime} \delta^{\prime} \beta^{\prime} \epsilon^{\prime}} u_{\gamma^{\prime}}\right) u^{\delta^{\prime}} u^{\epsilon^{\prime}}+\gamma_{\alpha^{\prime} \beta^{\prime} \gamma^{\prime}}^{\mathrm{tail}}
$$

where the tail term can be copied from Equation (500),

$$
\gamma_{\alpha^{\prime} \beta^{\prime} \gamma^{\prime}}^{\text {tail }}\left(x^{\prime}\right)=4 m \int_{-\infty}^{u^{-}} \nabla_{\gamma^{\prime}} G_{+\alpha^{\prime} \beta^{\prime} \mu \nu}\left(x^{\prime}, z\right) u^{\mu} u^{\nu} d \tau \text {. }
$$

The tensors that appear in Equation (545) all refer to the retarded point $x^{\prime} \equiv z(u)$, which can now be treated as an arbitrary point on the world line $\gamma$.

\subsubsection{Equations of motion}

The retarded gravitational field $\gamma_{\alpha \beta ; \gamma}$ of a point particle is singular on the world line, and this behaviour makes it difficult to understand how the field is supposed to act on the particle and influence its motion. The field's singularity structure was analyzed in Sections 5.3.3 and 5.3.4, and in Section 5.3.5 it was shown to originate from the singular field $\gamma_{\alpha \beta ; \gamma}^{\mathrm{S}}$; the radiative field $\gamma_{\alpha \beta ; \gamma}^{\mathrm{R}}$ was then shown to be smooth on the world line.

Living Reviews in Relativity

http://www. livingreviews . org/lrr-2004-6 
To make sense of the retarded field's action on the particle we can follow the discussions of Section 5.1.6 and 5.2.6 and postulate that the self gravitational field of the point particle is either $\left\langle\gamma_{\mu \nu ; \lambda}\right\rangle$, as worked out in Equation (523), or $\gamma_{\mu \nu ; \lambda}^{\mathrm{R}}$, as worked out in Equation (544). These regularized fields are both given by

$$
\gamma_{\mu \nu ; \lambda}^{\mathrm{reg}}=-4 m\left(u_{(\mu} R_{\nu) \rho \lambda \xi}+R_{\mu \rho \nu \xi} u_{\lambda}\right) u^{\rho} u^{\xi}+\gamma_{\mu \nu \lambda}^{\mathrm{tail}}
$$

and

$$
\gamma_{\mu \nu \lambda}^{\mathrm{tail}}=4 m \int_{-\infty}^{\tau^{-}} \nabla_{\lambda} G_{+\mu \nu \mu^{\prime} \nu^{\prime}}\left(z(\tau), z\left(\tau^{\prime}\right)\right) u^{\mu^{\prime}} u^{\nu^{\prime}} d \tau^{\prime}
$$

in which all tensors are now evaluated at an arbitrary point $z(\tau)$ on the world line $\gamma$.

The actual gravitational perturbation $h_{\alpha \beta}$ is obtained by inverting Equation (491), which leads to $h_{\mu \nu ; \lambda}=\gamma_{\mu \nu ; \gamma}-\frac{1}{2} g_{\mu \nu} \gamma_{\rho ; \lambda}^{\rho}$. Substituting Equation (546) yields

$$
h_{\mu \nu ; \lambda}^{\mathrm{reg}}=-4 m\left(u_{(\mu} R_{\nu) \rho \lambda \xi}+R_{\mu \rho \nu \xi} u_{\lambda}\right) u^{\rho} u^{\xi}+h_{\mu \nu \lambda}^{\mathrm{tail}},
$$

where the tail term is given by the trace-reversed counterpart to Equation (547):

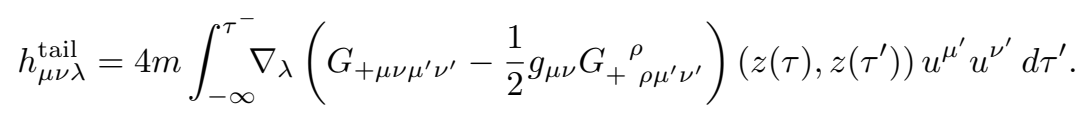

When this regularized field is substituted into Equation (494), we find that the terms that depend on the Riemann tensor cancel out, and we are left with

$$
\frac{D u^{\mu}}{d \tau}=-\frac{1}{2}\left(g^{\mu \nu}+u^{\mu} u^{\nu}\right)\left(2 h_{\nu \lambda \rho}^{\text {tail }}-h_{\lambda \rho \nu}^{\text {tail }}\right) u^{\lambda} u^{\rho} .
$$

We see that only the tail term is involved in the final form of the equations of motion. The tail integral of Equation (549) involves the current position $z(\tau)$ of the particle, at which all tensors with unprimed indices are evaluated, as well as all prior positions $z\left(\tau^{\prime}\right)$, at which all tensors with primed indices are evaluated. The tail integral is cut short at $\tau^{\prime}=\tau^{-} \equiv \tau-0^{+}$to avoid the singular behaviour of the retarded Green's function at coincidence; this limiting procedure was justified at the beginning of Section 5.3.3.

Equation (550) was first derived by Yasushi Mino, Misao Sasaki, and Takahiro Tanaka in 1997 [39]. (An incomplete treatment had been given previously by Morette-DeWitt and Ging [42].) An alternative derivation was then produced, also in 1997, by Theodore C. Quinn and Robert M. Wald [49]. These equations are now known as the MiSaTaQuWa equations of motion. It should be noted that Equation (550) is formally equivalent to the statement that the point particle moves on a geodesic in a spacetime with metric $g_{\alpha \beta}+h_{\alpha \beta}^{\mathrm{R}}$, where $h_{\alpha \beta}^{\mathrm{R}}$ is the radiative metric perturbation obtained by trace-reversal of the potentials $\gamma_{\alpha \beta}^{\mathrm{R}} \equiv \gamma_{\alpha \beta}-\gamma_{\alpha \beta}^{\mathrm{S}}$; this perturbed metric is smooth on the world line, and it is a solution to the vacuum field equations. This elegant interpretation of the MiSaTaQuWa equations was proposed in 2002 by Steven Detweiler and Bernard F. Whiting [23]. Quinn and Wald [50] have shown that under some conditions, the total work done by the gravitational self-force is equal to the energy radiated (in gravitational waves) by the particle.

\subsubsection{Gauge dependence of the equations of motion}

The equations of motion derived in the preceding Section 5.3.6 refer to a specific choice of gauge for the metric perturbation $h_{\alpha \beta}$ produced by a point particle of mass $m$. We indeed recall that back at Equation (492) we imposed the Lorenz gauge condition $\gamma_{; \beta}^{\alpha \beta}=0$ on the gravitational potentials $\gamma_{\alpha \beta} \equiv h_{\alpha \beta}-\frac{1}{2}\left(g^{\gamma \delta} h_{\gamma \delta}\right) g_{\alpha \beta}$. By virtue of this condition we found that the potentials 
satisfy the wave equation of Equation (493) in a background spacetime with metric $g_{\alpha \beta}$. The hyperbolic nature of this equation allowed us to identify the retarded solution as the physically relevant solution, and the equations of motion were obtained by removing the singular part of the retarded field. It seems clear that the Lorenz condition is a most appropriate choice of gauge.

Once the equations of motion have been formulated, however, the freedom of performing a gauge transformation (either away from the Lorenz gauge, or within the class of Lorenz gauges) should be explored. A gauge transformation will affect the form of the equations of motion: These must depend on the choice of coordinates, and there is no reason to expect Equation (550) to be invariant under a gauge transformation. Our purpose in this section is to work out how the equations of motion change under such a transformation. This issue was first examined by Barack and Ori [8].

We introduce a coordinate transformation of the form

$$
x^{\alpha} \rightarrow x^{\alpha}+\xi^{\alpha}
$$

where $x^{\alpha}$ are the coordinates of the background spacetime, and $\xi^{\alpha}$ is a vector field that we take to be of order $m$. We assume that $\xi^{\alpha}$ is smooth in a neighbourhood of the world line $\gamma$. The coordinate transformation changes the background metric according to

$$
g_{\alpha \beta} \rightarrow g_{\alpha \beta}-\xi_{\alpha ; \beta}-\xi_{\beta ; \alpha}+\mathcal{O}\left(m^{2}\right),
$$

and this change can be interpreted as a gauge transformation of the metric perturbation created by the moving particle:

$$
h_{\alpha \beta} \rightarrow h_{\alpha \beta}-\xi_{\alpha ; \beta}-\xi_{\beta ; \alpha}
$$

This, in turn, produces a change in the particle's acceleration,

$$
a^{\mu} \rightarrow a^{\mu}+a[\xi]^{\mu}
$$

where $a^{\mu}$ is the acceleration of Equation (550) and $a[\xi]^{\mu}$ is the "gauge acceleration" generated by the vector field $\xi^{\alpha}$.

To compute the gauge acceleration we substitute Equation (552) into Equation (494), and we simplify the result by invoking Ricci's identity, $\xi_{\lambda ; \nu \rho}-\xi_{\lambda ; \rho \nu}=R_{\nu \rho \omega \lambda} \xi^{\omega}$, and the fact that $a^{\mu}=\mathcal{O}(m)$. The final expression is

$$
a[\xi]^{\mu}=\left(\delta^{\mu}{ }_{\nu}+u^{\mu} u_{\nu}\right)\left(\frac{D^{2} \xi^{\nu}}{d \tau^{2}}+R_{\rho \omega \lambda}^{\nu} u^{\rho} \xi^{\omega} u^{\lambda}\right)
$$

where $D^{2} \xi^{\nu} / d \tau^{2}=\left(\xi_{; \mu}^{\nu} u^{\mu}\right)_{; \rho} u^{\rho}$ is the second covariant derivative of $\xi^{\nu}$ in the direction of the world line. The expression within the large brackets is familiar from the equation of geodesic deviation, which states that this quantity vanishes if $\xi^{\mu}$ is a deviation vector between two neighbouring geodesics. Equation (553), with $a[\xi]^{\mu}$ given by Equation (554), is therefore a generalized version of this statement.

\subsection{Motion of a small black hole}

\subsubsection{Matched asymptotic expansions}

The derivation of the MiSaTaQuWa equations of motion presented in Section 5.3 was framed within the paradigm introduced in Sections 5.1 and 5.2 to describe the motion of a point scalar charge, and a point electric charge, respectively. While this paradigm is well suited to fields that satisfy linear wave equations, it is not the best conceptual starting point in the nonlinear context of general relativity. The linearization of the Einstein field equations with respect to the small parameter $m$

Living Reviews in Relativity

http://www. livingreviews.org/lrr-2004-6 
did allow us to use the same mathematical techniques as in Sections 5.1 and 5.2, but the validity of the perturbative method must be critically examined when the gravitational potentials are allowed to be singular. So while Equation (550) does indeed give the correct equations of motion when $m$ is small, its previous derivation leaves much to be desired. In this section I provide another derivation that is entirely free of conceptual and technical pitfalls. Here the point mass will be replaced by a nonrotating black hole, and the perturbation's singular behaviour on the world line will be replaced by a well-behaved metric at the event horizon. We will use the powerful technique of matched asymptotic expansions [35, 31, 58, 19, 1, 20].

The problem presents itself with a clean separation of length scales, and the method relies entirely on this. On the one hand we have the length scale associated with the small black hole, which is set by its mass $m$. On the other hand we have the length scale associated with the background spacetime in which the black hole moves, which is set by the radius of curvature $\mathcal{R}$; formally this is defined so that a typical component of the background spacetime's Riemann tensor is equal to $1 / \mathcal{R}^{2}$ up to a numerical factor of order unity. We demand that $m / \mathcal{R} \ll 1$. As before we assume that the background spacetime contains no matter, so that its metric is a solution to the Einstein field equations in vacuum.

For example, suppose that our small black hole of mass $m$ is on an orbit of radius $b$ around another black hole of mass $M$. Then $\mathcal{R} \sim b \sqrt{b / M}>b$ and we take $m$ to be much smaller than the orbital separation. Notice that the time scale over which the background geometry changes is of the order of the orbital period $b \sqrt{b / M} \sim \mathcal{R}$, so that this does not constitute a separate scale. Similarly, the inhomogeneity scale - the length scale over which the Riemann tensor of the background spacetime changes - is of order $b \sim \mathcal{R} \sqrt{M / b}<\mathcal{R}$ and also does not constitute an independent scale. (In this discussion we have considered $b / M$ to be of order unity, so as to represent a strong-field, fast-motion situation.)

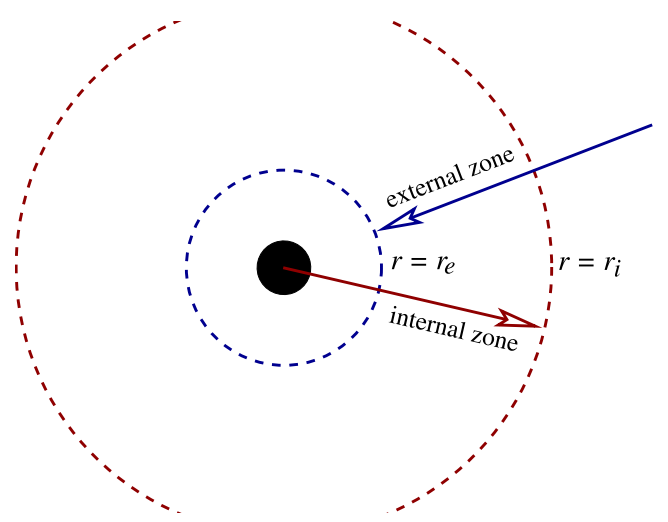

Figure 10: A black hole, represented by the black disk, is immersed in a background spacetime. The internal zone extends from $r=0$ to $r=r_{\mathrm{i}} \ll \mathcal{R}$, while the external zone extends from $r=r_{\mathrm{e}} \gg m$ to $r=\infty$. When $m \ll \mathcal{R}$ there exists a buffer zone that extends from $r=r_{\mathrm{e}}$ to $r=r_{\mathrm{i}}$. In the buffer zone $m / r$ and $r / \mathcal{R}$ are both small.

Let $r$ be a meaningful measure of distance from the small black hole, and let us consider a region of spacetime defined by $r<r_{\mathrm{i}}$, where $r_{\mathrm{i}}$ is a constant that is much smaller than $\mathcal{R}$. This inequality defines a narrow world tube that surrounds the small black hole, and we shall call this region the internal zone (see Figure 10). In the internal zone the gravitational field is dominated by the black hole, and the metric can be expressed as

$$
\mathrm{g}(\text { internal zone })=g(\text { black hole })+H_{1} / \mathcal{R}+H_{2} / \mathcal{R}^{2}+\ldots,
$$


where $g$ (black hole) is the metric of a nonrotating black hole in isolation (as given by the unperturbed Schwarzschild solution), while $H_{1}$ and $H_{2}$ are corrections associated with the conditions in the external universe. The metric of Equation (555) represents a black hole that is distorted by the tidal gravitational field of the external universe, and $H_{1}, H_{2}$ are functions of $m$ and the spacetime coordinates that can be obtained by solving the Einstein field equations. They must be such that the spacetime possesses a regular event horizon near $r=2 m$, and such that $\mathrm{g}$ (internal zone) agrees with the metric of the external universe - the metric of the background spacetime in the absence of the black hole - when $r \gg m$. As we shall see in Section 5.4.2, $H_{1}$ actually vanishes and the small correction $H_{2} / \mathcal{R}^{2}$ can be obtained by employing the well-developed tools of black-hole perturbation theory [51, 59, 63].

Consider now a region of spacetime defined by $r>r_{\mathrm{e}}$, where $r_{\mathrm{e}}$ is a constant that is much larger than $m$; this region will be called the external zone (see Figure 10). In the external zone the gravitational field is dominated by the conditions in the external universe, and the metric can be expressed as

$$
\mathrm{g}(\text { external zone })=g(\text { background spacetime })+m h_{1}+m^{2} h_{2}+\ldots,
$$

where $g$ (background spacetime) is the unperturbed metric of the background spacetime in which the black hole is moving, while $h_{1}$ and $h_{2}$ are corrections associated with the hole's presence; these are functions of $\mathcal{R}$ and the spacetime coordinates that can be obtained by solving the Einstein field equations. We shall truncate Equation (556) to first order in $m$, and $m h_{1}$ will be calculated in Section 5.4.3 by linearizing the field equations about the metric of the background spacetime. In the external zone the perturbation associated with the presence of a black hole cannot be distinguished from the perturbation produced by a point particle of the same mass, and $m h_{1}$ will therefore be obtained by solving Equation (493) in the background spacetime.

The metric g(external zone) returned by the procedure described in the preceding paragraph is a functional of a world line $\gamma$ that represents the motion of the small black hole in the background spacetime. Our goal is to obtain a description of this world line, in the form of equations of motion to be satisfied by the black hole; these equations will be formulated in the background spacetime. It is important to understand that fundamentally, $\gamma$ exists only as an external-zone construct: It is only in the external zone that the black hole can be thought of as moving on a world line; in the internal zone the black hole is revealed as an extended object and the notion of a world line describing its motion is no longer meaningful.

Equations (555) and (556) give two different expressions for the metric of the same spacetime; the first is valid in the internal zone $r<r_{\mathrm{i}} \ll \mathcal{R}$, while the second is valid in the external zone $r>r_{\mathrm{e}} \gg m$. The fact that $\mathcal{R} \gg m$ allows us to define a buffer zone in which $r$ is restricted to the interval $r_{\mathrm{e}}<r<r_{\mathrm{i}}$. In the buffer zone $r$ is simultaneously much larger than $m$ and much smaller than $\mathcal{R}$ - a typical value might be $\sqrt{m \mathcal{R}}$ - and Equations $(555,556)$ are simultaneously valid. Since the two metrics are the same up to a diffeomorphism, these expressions must agree. And since $\mathrm{g}$ (external zone) is a functional of a world line $\gamma$ while $\mathrm{g}$ (internal zone) contains no such information, matching the metrics necessarily determines the motion of the small black hole in the background spacetime. What we have here is a beautiful implementation of the general observation that the motion of self-gravitating bodies is determined by the Einstein field equations.

It is not difficult to recognize that the metrics of Equations $(555,556)$ can be matched in the buffer zone. When $r \gg m$ in the internal zone, the metric of the unperturbed black hole can be expanded as $g$ (black hole) $=\eta \oplus m / r \oplus m^{2} / r^{2} \oplus \ldots$, where $\eta$ is the metric of flat spacetime (in asymptotically inertial coordinates) and the symbol $\oplus$ means "and a term of the form...". On the other hand, dimensional analysis dictates that $H_{1} / \mathcal{R}$ be of the form $r / \mathcal{R} \oplus m / \mathcal{R} \oplus m^{2} /(r \mathcal{R}) \oplus \ldots$

Living Reviews in Relativity

http://www . livingreviews . org//rr-2004-6 
while $H_{2} / \mathcal{R}^{2}$ should be expressed as $r^{2} / \mathcal{R}^{2} \oplus m r / \mathcal{R}^{2} \oplus m^{2} / \mathcal{R}^{2} \oplus \ldots$ Altogether we obtain

$$
\begin{aligned}
\mathrm{g}(\text { buffer zone })= & \eta \oplus m / r \oplus m^{2} / r^{2} \oplus \ldots \\
& \oplus r / \mathcal{R} \oplus m / \mathcal{R} \oplus m^{2} /(r \mathcal{R}) \oplus \ldots \\
& \oplus r^{2} / \mathcal{R}^{2} \oplus m r / \mathcal{R}^{2} \oplus m^{2} / \mathcal{R}^{2} \oplus \ldots \\
& \oplus \ldots
\end{aligned}
$$

for the buffer-zone metric. If instead we approach the buffer zone from the opposite side, letting $r$ be much smaller than $\mathcal{R}$ in the external zone, we have that the metric of the background spacetime can be expressed as $g$ (background spacetime $)=\eta \oplus r / \mathcal{R} \oplus r^{2} / \mathcal{R}^{2} \oplus \ldots$, where the expansion now uses world-line based coordinates such as the Fermi normal coordinates of Section 3.2 or the retarded coordinates of Section 3.3. On dimensional grounds we also have $m h_{1}=m / r \oplus m / \mathcal{R} \oplus m r / \mathcal{R}^{2} \oplus \ldots$ and $m^{2} h_{2}=m^{2} / r^{2} \oplus m^{2} /(r \mathcal{R}) \oplus m^{2} / \mathcal{R}^{2} \oplus \ldots$ Altogether this gives

$$
\begin{aligned}
\mathrm{g}(\text { buffer zone })= & \eta \oplus r / \mathcal{R} \oplus r^{2} / \mathcal{R}^{2} \oplus \ldots \\
& \oplus m / r \oplus m / \mathcal{R} \oplus m r / \mathcal{R}^{2} \oplus \ldots \\
& \oplus m^{2} / r^{2} \oplus m^{2} /(r \mathcal{R}) \oplus m^{2} / \mathcal{R}^{2} \oplus \ldots \\
& \oplus \ldots
\end{aligned}
$$

for the buffer-zone metric. Apart from a different ordering of terms, the metrics of Equations (557) and (558) have identical forms.

Matching the metrics of Equations (555) and (556) in the buffer zone can be carried out in practice only after performing a transformation from the external coordinates used to express $\mathrm{g}$ (external zone) to the internal coordinates employed for $\mathrm{g}$ (internal zone). The details of this coordinate transformation will be described in Section 5.4.4, and the end result of matching - the MiSaTaQuWa equations of motion - will be revealed in Section 5.4.5.

\subsubsection{Metric in the internal zone}

To flesh out the ideas contained in the previous Section 5.4.1 we first calculate the internal-zone metric and replace Equation (555) by a more concrete expression. We recall that the internal zone is defined by $r<r_{\mathrm{i}} \ll \mathcal{R}$, where $r$ is a suitable measure of distance from the black hole.

We begin by expressing $g$ (black hole), the Schwarzschild metric of an isolated black hole of mass $m$, in terms of retarded Eddington-Finkelstein coordinates $\left(\bar{u}, \bar{r}, \bar{\theta}^{A}\right)$, where $\bar{u}$ is retarded time, $\bar{r}$ the usual areal radius, and $\bar{\theta}^{A}=(\bar{\theta}, \bar{\phi})$ are two angles spanning the two-spheres of constant $\bar{u}$ and $\bar{r}$. The metric is given by

$$
d s^{2}=-f d \bar{u}^{2}-2 d \bar{u} d \bar{r}+\bar{r}^{2} d \bar{\Omega}^{2}, \quad f=1-\frac{2 m}{\bar{r}},
$$

where $d \bar{\Omega}^{2}=\bar{\Omega}_{A B} d \bar{\theta}^{A} d \bar{\theta}^{B}=d \bar{\theta}^{2}+\sin ^{2} \bar{\theta} d \bar{\phi}^{2}$ is the line element on the unit two-sphere. In the limit $r \gg m$ this metric achieves the asymptotic values

$$
g_{\bar{u} \bar{u}} \rightarrow-1, \quad g_{\bar{u} \bar{r}}=-1, \quad g_{\bar{u} \bar{A}}=0, \quad g_{\bar{A} \bar{B}}=\bar{r}^{2} \bar{\Omega}_{A B} ;
$$

these are appropriate for a black hole immersed in a flat spacetime charted by retarded coordinates.

The corrections $H_{1}$ and $H_{2}$ in Equation (555) encode the information that our black hole is not isolated but in fact immersed in an external universe whose metric becomes $g$ (background spacetime) asymptotically. In the internal zone the metric of the background spacetime can be expanded in powers of $\bar{r} / \mathcal{R}$ and expressed in a form that can be directly imported from Section 3.3. If we assume for the moment that the "world line" $\bar{r}=0$ has no acceleration in the background spacetime 
(a statement that will be justified shortly), then the asymptotic values of $g$ (internal zone) must be given by Equations $(210,211,212,213)$ :

$$
\begin{aligned}
\mathrm{g}_{\bar{u} \bar{u}} \rightarrow-1-\bar{r}^{2} \overline{\mathcal{E}}^{*}+\mathcal{O}\left(\bar{r}^{3} / \mathcal{R}^{3}\right), & \mathrm{g}_{\bar{u} \bar{r}}=-1, \\
\mathrm{~g}_{\bar{u} \bar{A}} \rightarrow \frac{2}{3} \bar{r}^{3}\left(\overline{\mathcal{E}}_{A}^{*}+\overline{\mathcal{B}}_{A}^{*}\right)+\mathcal{O}\left(\bar{r}^{4} / \mathcal{R}^{3}\right), & \mathrm{g}_{\bar{A} \bar{B}} \rightarrow \bar{r}^{2} \bar{\Omega}_{A B}-\frac{1}{3} \bar{r}^{4}\left(\overline{\mathcal{E}}_{A B}^{*}+\overline{\mathcal{B}}_{A B}^{*}\right)+\mathcal{O}\left(\bar{r}^{5} / \mathcal{R}^{3}\right),
\end{aligned}
$$

where

$$
\overline{\mathcal{E}}^{*}=\mathcal{E}_{a b} \bar{\Omega}^{a} \bar{\Omega}^{b}, \quad \overline{\mathcal{E}}_{A}^{*}=\mathcal{E}_{a b} \bar{\Omega}_{A}^{a} \bar{\Omega}^{b}, \quad \overline{\mathcal{E}}_{A B}^{*}=2 \mathcal{E}_{a b} \bar{\Omega}_{A}^{a} \bar{\Omega}_{B}^{b}+\overline{\mathcal{E}}^{*} \bar{\Omega}_{A B}
$$

and

$$
\overline{\mathcal{B}}_{A}^{*}=\varepsilon_{a b c} \bar{\Omega}_{A}^{a} \bar{\Omega}^{b} \mathcal{B}_{d}^{c} \bar{\Omega}^{d}, \quad \overline{\mathcal{B}}_{A B}^{*}=2 \varepsilon_{a c d} \bar{\Omega}^{c} \mathcal{B}_{b}^{d} \bar{\Omega}_{A}^{(a} \bar{\Omega}_{B}^{b)}
$$

are the tidal gravitational fields that were first introduced in Section 3.3.8. Recall that $\bar{\Omega}^{a}=$ $(\sin \bar{\theta} \cos \bar{\phi}, \sin \bar{\theta} \sin \bar{\phi}$

$\cos \bar{\theta}$ ) and $\bar{\Omega}_{A}^{a}=\partial \bar{\Omega}^{a} / \partial \bar{\theta}^{A}$. Apart from an angular dependence made explicit by these relations, the tidal fields depend on $\bar{u}$ through the frame components $\mathcal{E}_{a b} \equiv R_{a 0 b 0}=\mathcal{O}\left(1 / \mathcal{R}^{2}\right)$ and $\mathcal{B}_{b}^{a} \equiv \frac{1}{2} \varepsilon^{a c d} R_{0 b c d}=\mathcal{O}\left(1 / \mathcal{R}^{2}\right)$ of the Riemann tensor. (This is the Riemann tensor of the background spacetime evaluated at $\bar{r}=0$.) Notice that we have incorporated the fact that the Ricci tensor vanishes at $\bar{r}=0$ : The black hole moves in a vacuum spacetime.

The modified asymptotic values lead us to the following ansatz for the internal-zone metric:

$$
\begin{aligned}
\mathrm{g}_{\bar{u} \bar{u}} & =-f\left[1+\bar{r}^{2} e_{1}(\bar{r}) \overline{\mathcal{E}}^{*}\right]+\mathcal{O}\left(\bar{r}^{3} / \mathcal{R}^{3}\right), \\
\mathrm{g}_{\bar{u} \bar{r}} & =-1, \\
\mathrm{~g}_{\bar{u} \bar{A}} & =\frac{2}{3} \bar{r}^{3}\left[e_{2}(\bar{r}) \overline{\mathcal{E}}_{A}^{*}+b_{2}(\bar{r}) \overline{\mathcal{B}}_{A}^{*}\right]+\mathcal{O}\left(\bar{r}^{4} / \mathcal{R}^{3}\right), \\
\mathrm{g}_{\bar{A} \bar{B}} & =\bar{r}^{2} \bar{\Omega}_{A B}-\frac{1}{3} \bar{r}^{4}\left[e_{3}(\bar{r}) \overline{\mathcal{E}}_{A B}^{*}+b_{3}(\bar{r}) \overline{\mathcal{B}}_{A B}^{*}\right]+\mathcal{O}\left(\bar{r}^{5} / \mathcal{R}^{3}\right) .
\end{aligned}
$$

The five unknown functions $e_{1}, e_{2}, e_{3}, b_{2}$, and $b_{3}$ can all be determined by solving the Einstein field equations; they must all approach unity when $r \gg m$ and be well-behaved at $r=2 m$ (so that the tidally distorted black hole will have a nonsingular event horizon). It is clear from Equations (562, $563,564,565)$ that the assumed deviation of $\mathrm{g}$ (internal zone) with respect to $g$ (black hole) scales as $1 / \mathcal{R}^{2}$. It is therefore of the form of Equation (555) with $H_{1}=0$. The fact that $H_{1}$ vanishes comes as a consequence of our previous assumption that the "world line" $\bar{r}=0$ has a zero acceleration in the background spacetime; a nonzero acceleration of order $1 / \mathcal{R}$ would bring terms of order $1 / \mathcal{R}$ to the metric, and $H_{1}$ would then be nonzero.

Why is the assumption of no acceleration justified? As I shall explain in the next paragraph (and you might also refer back to the discussion of Section 5.3.7), the reason is simply that it reflects a choice of coordinate system: Setting the acceleration to zero amounts to adopting a specific - and convenient - gauge condition. This gauge differs from the Lorenz gauge adopted in Section 5.3, and it will be our choice in this section only; in the following Section 5.4.3 we will return to the Lorenz gauge, and the acceleration will be seen to return to its standard MiSaTaQuWa expression.

Inspection of Equations (560) and (561) reveals that the angular dependence of the metric perturbation is generated entirely by scalar, vectorial, and tensorial spherical harmonics of degree $\ell=2$. In particular, $H_{2}$ contains no $\ell=0$ and $\ell=1$ modes, and this statement reflects a choice of gauge condition. Zerilli has shown [63] that a perturbation of the Schwarzschild spacetime with $\ell=0$ corresponds to a shift in the mass parameter. As Thorne and Hartle have shown [58], a black hole interacting with its environment will undergo a change of mass, but this effect is of order $m^{3} / \mathcal{R}^{2}$ and thus beyond the level of accuracy of our calculations. There is therefore no need to include $\ell=0$ terms in $H_{2}$. Similarly, it was shown by Zerilli that odd-parity perturbations of degree $\ell=1$ correspond to a shift in the black hole's angular-momentum parameters. As Thorne and

Living Reviews in Relativity

http://www. livingreviews . org/lrr-2004-6 
Hartle have shown, a change of angular momentum is quadratic in the hole's angular momentum, and we can ignore this effect when dealing with a nonrotating black hole. There is therefore no need to include odd-parity, $\ell=1$ terms in $H_{2}$. Finally, Zerilli has shown that in a vacuum spacetime, even-parity perturbations of degree $\ell=1$ correspond to a change of coordinate system - these modes are pure gauge. Since we have the freedom to adopt any gauge condition, we can exclude even-parity, $\ell=1$ terms from the perturbed metric. This leads us to Equations (562, 563, 564, $565)$, which contain only $\ell=2$ perturbation modes; the even-parity modes are contained in those terms that involve $\mathcal{E}_{a b}$, while the odd-parity modes are associated with $\mathcal{B}_{a b}$. The perturbed metric contains also higher multipoles, but those come at a higher order in $1 / \mathcal{R}$; for example, the terms of order $1 / \mathcal{R}^{3}$ include $\ell=3$ modes. We conclude that Equations $(562,563,564,565)$ is a sufficiently general ansatz for the perturbed metric in the internal zone.

There remains the task of finding the functions $e_{1}, e_{2}, e_{3}, b_{2}$, and $b_{3}$. For this it is sufficient to take, say, $\mathcal{E}_{12}=\mathcal{E}_{21}$ and $\mathcal{B}_{12}=\mathcal{B}_{21}$ as the only nonvanishing components of the tidal fields $\mathcal{E}_{a b}$ and $\mathcal{B}_{a b}$. And since the equations for even-parity and odd-parity perturbations decouple, each case can be considered separately. Including only even-parity perturbations, Equations (562)-(565) become

$$
\begin{array}{ll}
\mathrm{g}_{\bar{u} \bar{u}}=-f\left(1+\bar{r}^{2} e_{1} \mathcal{E}_{12} \sin ^{2} \bar{\theta} \sin 2 \bar{\phi}\right), & \mathrm{g}_{\bar{u} \bar{r}}=-1, \\
\mathrm{~g}_{\bar{u} \bar{\theta}}=\frac{2}{3} \bar{r}^{3} e_{2} \mathcal{E}_{12} \sin \bar{\theta} \cos \bar{\theta} \sin 2 \bar{\phi}, & \mathrm{g}_{\bar{u} \bar{\phi}}=\frac{2}{3} \bar{r}^{3} e_{2} \mathcal{E}_{12} \sin ^{2} \bar{\theta} \cos 2 \bar{\phi}, \\
\mathrm{g}_{\bar{\theta} \bar{\theta}}=\bar{r}^{2}-\frac{1}{3} \bar{r}^{4} e_{3} \mathcal{E}_{12}\left(1+\cos ^{2} \bar{\theta}\right) \sin 2 \bar{\phi}, & \mathrm{g}_{\bar{\theta} \bar{\phi}}=-\frac{2}{3} \bar{r}^{4} e_{3} \mathcal{E}_{12} \sin \bar{\theta} \cos \bar{\theta} \cos 2 \bar{\phi}, \\
\mathrm{g}_{\bar{\phi} \bar{\phi}}=\bar{r}^{2} \sin ^{2} \bar{\theta}+\frac{1}{3} \bar{r}^{4} e_{3} \mathcal{E}_{12} \sin ^{2} \bar{\theta}\left(1+\cos ^{2} \bar{\theta}\right) \sin 2 \bar{\phi} . &
\end{array}
$$

This metric is then substituted into the vacuum Einstein field equations, $\mathrm{G}_{\alpha \beta}=0$. Calculating the Einstein tensor is simplified by linearizing with respect to $\mathcal{E}_{12}$ and discarding its derivatives with respect to $\bar{u}$ : Since the time scale over which $\mathcal{E}_{a b}$ changes is of order $\mathcal{R}$, the ratio between temporal and spatial derivatives is of order $\bar{r} / \mathcal{R}$ and therefore small in the internal zone; the temporal derivatives can be consistently neglected. The field equations produce ordinary differential equations to be satisfied by the functions $e_{1}, e_{2}$, and $e_{3}$. Those are easily decoupled, and demanding that the functions all approach unity as $r \rightarrow \infty$ and be well-behaved at $r=2 m$ yields the unique solutions

$$
e_{1}(\bar{r})=e_{2}(\bar{r})=f, \quad e_{3}(\bar{r})=1-\frac{2 m^{2}}{\bar{r}^{2}} .
$$

Switching now to odd-parity perturbations, Equations $(562,563,564,565)$ become

$$
\begin{gathered}
\mathrm{g}_{\bar{u} \bar{u}}=-f \quad \mathrm{~g}_{\bar{u} \bar{r}}=-1, \quad \mathrm{~g}_{\bar{u} \bar{\theta}}=-\frac{2}{3} \bar{r}^{3} b_{2} \mathcal{B}_{12} \sin \bar{\theta} \cos 2 \bar{\phi}, \quad \mathrm{g}_{\bar{u} \bar{\phi}}=\frac{2}{3} \bar{r}^{3} b_{2} \mathcal{B}_{12} \sin ^{2} \bar{\theta} \cos \bar{\theta} \sin 2 \bar{\phi}, \\
\mathrm{g}_{\bar{\theta} \bar{\theta}}=\bar{r}^{2}+\frac{2}{3} \bar{r}^{4} b_{3} \mathcal{B}_{12} \cos \bar{\theta} \cos 2 \bar{\phi}, \quad \mathrm{g}_{\bar{\theta} \bar{\phi}}=-\frac{1}{3} \bar{r}^{4} b_{3} \mathcal{B}_{12} \sin \bar{\theta}\left(1+\cos ^{2} \bar{\theta}\right) \sin 2 \bar{\phi}, \\
\mathrm{g}_{\bar{\phi} \bar{\phi}}=\bar{r}^{2} \sin ^{2} \bar{\theta}-\frac{2}{3} \bar{r}^{4} b_{3} \mathcal{B}_{12} \sin ^{2} \bar{\theta} \cos \bar{\theta} \cos 2 \bar{\phi} .
\end{gathered}
$$

Following the same procedure, we arrive at

$$
b_{2}(\bar{r})=f, \quad b_{3}(\bar{r})=1 .
$$

Substituting Equations (566) and (567) into Equations (562, 563, 564, 565) returns our final expression for the metric in the internal zone.

It shall prove convenient to transform $\mathrm{g}$ (internal zone) from the quasi-spherical coordinates $\left(\bar{r}, \bar{\theta}^{A}\right)$ to a set of quasi-Cartesian coordinates $\bar{x}^{a}=\bar{r} \bar{\Omega}^{a}\left(\bar{\theta}^{A}\right)$. The transformation rules are worked 
out in Section 3.3.7 and further illustrated in Section 3.3.8. This gives

$$
\begin{aligned}
& \mathrm{g}_{\bar{u} \bar{u}}=-f\left(1+\bar{r}^{2} f \overline{\mathcal{E}}^{*}\right)+\mathcal{O}\left(\bar{r}^{3} / \mathcal{R}^{3}\right), \\
& \mathrm{g}_{\bar{u} \bar{a}}=-\bar{\Omega}_{a}+\frac{2}{3} \bar{r}^{2} f\left(\overline{\mathcal{E}}_{a}^{*}+\overline{\mathcal{B}}_{a}^{*}\right)+\mathcal{O}\left(\bar{r}^{3} / \mathcal{R}^{3}\right), \\
& \mathrm{g}_{\bar{a} \bar{b}}=\delta_{a b}-\bar{\Omega}_{a} \bar{\Omega}_{b}-\frac{1}{3} \bar{r}^{2}\left(1-2 \frac{m^{2}}{\bar{r}^{2}}\right) \overline{\mathcal{E}}_{a b}^{*}-\frac{1}{3} \bar{r}^{2} \overline{\mathcal{B}}_{a b}^{*}+\mathcal{O}\left(\bar{r}^{3} / \mathcal{R}^{3}\right),
\end{aligned}
$$

where $f=1-2 m / \bar{r}$ and where the tidal fields

$$
\begin{aligned}
\overline{\mathcal{E}}^{*} & =\mathcal{E}_{a b} \bar{\Omega}^{a} \bar{\Omega}^{b} \\
\overline{\mathcal{E}}_{a}^{*} & =\left(\delta_{a}{ }^{b}-\bar{\Omega}_{a} \bar{\Omega}^{b}\right) \mathcal{E}_{b c} \bar{\Omega}^{c}, \\
\overline{\mathcal{E}}_{a b}^{*} & =2 \mathcal{E}_{a b}-2 \bar{\Omega}_{a} \mathcal{E}_{b c} \bar{\Omega}^{c}-2 \bar{\Omega}_{b} \mathcal{E}_{a c} \bar{\Omega}^{c}+\left(\delta_{a b}+\bar{\Omega}_{a} \bar{\Omega}_{b}\right) \overline{\mathcal{E}}^{*}, \\
\overline{\mathcal{B}}_{a}^{*} & =\varepsilon_{a b c} \bar{\Omega}^{b} \mathcal{B}^{c} \bar{\Omega}_{d}^{d}, \\
\overline{\mathcal{B}}_{a b}^{*} & =\varepsilon_{a c d} \bar{\Omega}^{c} \mathcal{B}^{d}{ }_{e}\left(\delta_{b}^{e}-\bar{\Omega}^{e} \bar{\Omega}_{b}\right)+\varepsilon_{b c d} \bar{\Omega}^{c} \mathcal{B}^{d}{ }_{e}\left(\delta^{e}{ }_{a}-\bar{\Omega}^{e} \bar{\Omega}_{a}\right)
\end{aligned}
$$

were first introduced in Section 3.3.8. The metric of Equations $(568,569,570)$ represents the spacetime geometry of a black hole immersed in an external universe and distorted by its tidal gravitational fields.

\subsubsection{Metric in the external zone}

We next move on to the external zone and seek to replace Equation (556) by a more concrete expression; recall that the external zone is defined by $m \ll r_{\mathrm{e}}<r$. As was pointed out in Section 5.4.1, in the external zone the gravitational perturbation associated with the presence of a black hole cannot be distinguished from the perturbation produced by a point particle of the same mass; it can therefore be obtained by solving Equation (493) in a background spacetime with metric $g$ (background spacetime). The external-zone metric is decomposed as

$$
\mathrm{g}_{\alpha \beta}=g_{\alpha \beta}+h_{\alpha \beta},
$$

where $g_{\alpha \beta}$ is the metric of the background spacetime and $h_{\alpha \beta}=\mathcal{O}(m)$ is the perturbation; we shall work consistently to first order in $m$ and systematically discard all terms of higher order. We relate $h_{\alpha \beta}$ to trace-reversed potentials $\gamma_{\alpha \beta}$,

$$
h_{\alpha \beta}=\gamma_{\alpha \beta}-\frac{1}{2}\left(g^{\gamma \delta} \gamma_{\gamma \delta}\right) g_{\alpha \beta},
$$

and solving the linearized field equations produces

$$
\gamma_{\alpha \beta}(x)=4 m \int_{\gamma} G_{+\alpha \beta \mu \nu}(x, z) u^{\mu} u^{\nu} d \tau,
$$

where $z^{\mu}(\tau)$ gives the description of the world line $\gamma, \tau$ is proper time in the background spacetime, $u^{\mu}=d z^{\mu} / d \tau$ is the four-velocity, and $G_{+}^{\alpha \beta}(x, z)$ is the retarded Green's function associated with Equation (493); the potentials of Equation (578) satisfy the Lorenz-gauge condition $\gamma_{; \beta}^{\alpha \beta}=0$. As was pointed out in Section 5.4.1, $\gamma_{\alpha \beta}$ (and therefore $h_{\alpha \beta}$ ) are functionals of a world line $\gamma$ that will be determined by matching $\mathrm{g}$ (external zone) to $\mathrm{g}$ (internal zone).

We now place ourselves in the buffer zone (where $m \ll r \ll \mathcal{R}$ and where the matching will take place) and work toward expressing $\mathrm{g}$ (external zone) as an expansion in powers of $r / \mathcal{R}$. For this purpose we will adopt the retarded coordinates $\left(u, r \Omega^{a}\right)$ of Section 3.3 and rely on the machinery developed there.

Living Reviews in Relativity

http://www.livingreviews . org/lrr-2004-6 
We begin with $g_{\alpha \beta}$, the metric of the background spacetime. We have seen in Section 3.3.8 that if the world line $\gamma$ is a geodesic, if the vectors $e_{a}^{\mu}$ are parallel transported on the world line, and if the Ricci tensor vanishes on $\gamma$, then the metric takes the form given by Equations (207, 208, 209). This form, however, is too restrictive for our purposes: We must allow $\gamma$ to have an acceleration, and allow the basis vectors to be transported in the most general way compatible with their orthonormality property; this transport law is given by Equation (138),

$$
\frac{D e_{a}^{\mu}}{d \tau}=a_{a} u^{\mu}+\omega_{a}^{b} e_{b}^{\mu}
$$

where $a_{a}(\tau)=a_{\mu} e_{a}^{\mu}$ are the frame components of the acceleration vector $a^{\mu}=D u^{\mu} / d \tau$, and $\omega_{a b}(\tau)=-\omega_{b a}(\tau)$ is a rotation tensor to be determined. Anticipating that $a_{a}$ and $\omega_{a b}$ will both be proportional to $m$, we express the metric of the background spacetime as

$$
\begin{aligned}
& g_{u u}=-1-2 r a_{a} \Omega^{a}-r^{2} \mathcal{E}^{*}+\mathcal{O}\left(r^{3} / \mathcal{R}^{3}\right), \\
& g_{u a}=-\Omega_{a}+r\left(\delta_{a}{ }^{b}-\Omega_{a} \Omega^{b}\right) a_{b}-r \omega_{a b} \Omega^{b}+\frac{2}{3} r^{2}\left(\mathcal{E}_{a}^{*}+\mathcal{B}_{a}^{*}\right)+\mathcal{O}\left(r^{3} / \mathcal{R}^{3}\right), \\
& g_{a b}=\delta_{a b}-\Omega_{a} \Omega_{b}-\frac{1}{3} r^{2}\left(\mathcal{E}_{a b}^{*}+\mathcal{B}_{a b}^{*}\right)+\mathcal{O}\left(r^{3} / \mathcal{R}^{3}\right),
\end{aligned}
$$

where $\mathcal{E}^{*}, \mathcal{E}_{a}^{*}, \mathcal{E}_{a b}^{*}, \mathcal{B}_{a}^{*}$, and $\mathcal{B}_{a b}^{*}$ are the tidal gravitational fields first introduced in Section 3.3.8. The metric of Equations $(580,581,582)$ is obtained from the general form of Equations $(171,172$, 173 ) by neglecting quadratic terms in $a_{a}$ and $\omega_{a b}$ and specializing to a zero Ricci tensor.

To express the perturbation $h_{\alpha \beta}$ as an expansion in powers of $r / \mathcal{R}$ we first go back to Equation (498) and rewrite it in the form

$$
\gamma_{\alpha \beta}(x)=\frac{4 m}{r} U_{\alpha \beta \gamma^{\prime} \delta^{\prime}}\left(x, x^{\prime}\right) u^{\gamma^{\prime}} u^{\delta^{\prime}}+\gamma_{\alpha \beta}^{\mathrm{tail}}(x),
$$

in which primed indices refer to the retarded point $x^{\prime} \equiv z(u)$ associated with $x$, and

$$
\begin{aligned}
\gamma_{\alpha \beta}^{\mathrm{tail}}(x) & =4 m \int_{\tau_{<}}^{u} V_{\alpha \beta \mu \nu}(x, z) u^{\mu} u^{\nu} d \tau+4 m \int_{-\infty}^{\tau_{<}} G_{+\alpha \beta \mu \nu}(x, z) u^{\mu} u^{\nu} d \tau \\
& \equiv 4 m \int_{-\infty}^{u^{-}} G_{+\alpha \beta \mu \nu}(x, z) u^{\mu} u^{\nu} d \tau
\end{aligned}
$$

is the "tail part" of the gravitational potentials (recall that $\tau_{<}$is the proper time at which $\gamma$ enters $x$ 's normal convex neighbourhood from the past). We next expand the first term on the righthand side of Equation (583) with the help of Equation (501), and the tail term is expanded using Equation (93) in which we substitute Equation (504) and the familiar relation $\sigma^{\alpha^{\prime}}=-r\left(u^{\alpha^{\prime}}+\right.$ $\left.\Omega^{a} e_{a}^{\alpha^{\prime}}\right)$. This gives

$$
\gamma_{\alpha \beta}(x)=g_{\alpha}^{\alpha^{\prime}} g_{\beta}^{\beta^{\prime}}\left[\frac{4 m}{r} u_{\alpha^{\prime}} u_{\beta^{\prime}}+\gamma_{\alpha^{\prime} \beta^{\prime}}^{\mathrm{tail}}+r \gamma_{\alpha^{\prime} \beta^{\prime} \gamma^{\prime}}^{\mathrm{tail}}\left(u^{\gamma^{\prime}}+\Omega^{c} e_{c}^{\gamma^{\prime}}\right)+\mathcal{O}\left(m r^{2} / \mathcal{R}^{3}\right)\right],
$$

where $\gamma_{\alpha^{\prime} \beta^{\prime}}^{\text {tail }}$ is the tensor of Equation (584) evaluated at $x^{\prime}$, and where

$$
\gamma_{\alpha^{\prime} \beta^{\prime} \gamma^{\prime}}^{\mathrm{tail}}\left(x^{\prime}\right)=4 m \int_{-\infty}^{u^{-}} \nabla_{\gamma^{\prime}} G_{+\alpha^{\prime} \beta^{\prime} \mu \nu}\left(x^{\prime}, z\right) u^{\mu} u^{\nu} d \tau
$$

was first defined by Equation (545). 
At this stage we introduce the trace-reversed fields

$$
\begin{aligned}
h_{\alpha^{\prime} \beta^{\prime}}^{\mathrm{tail}}\left(x^{\prime}\right) & =4 m \int_{-\infty}^{u^{-}}\left(G_{+\alpha^{\prime} \beta^{\prime} \mu \nu}-\frac{1}{2} g_{\alpha^{\prime} \beta^{\prime}} G_{+\delta^{\prime} \mu \nu} \delta^{\prime}\right)\left(x^{\prime}, z\right) u^{\mu} u^{\nu} d \tau, \\
h_{\alpha^{\prime} \beta^{\prime} \gamma^{\prime}}^{\text {tail }}\left(x^{\prime}\right) & =4 m \int_{-\infty}^{u^{-}} \nabla_{\gamma^{\prime}}\left(G_{+\alpha^{\prime} \beta^{\prime} \mu \nu}-\frac{1}{2} g_{\alpha^{\prime} \beta^{\prime}} G_{+\delta^{\prime} \mu \nu}^{\delta^{\prime}}\right)\left(x^{\prime}, z\right) u^{\mu} u^{\nu} d \tau
\end{aligned}
$$

and recognize that the metric perturbation obtained from Equations (577) and (585) is

$$
h_{\alpha \beta}(x)=g_{\alpha}^{\alpha^{\prime}} g_{\beta}^{\beta^{\prime}}\left[\frac{2 m}{r}\left(2 u_{\alpha^{\prime}} u_{\beta^{\prime}}+g_{\alpha^{\prime} \beta^{\prime}}\right)+h_{\alpha^{\prime} \beta^{\prime}}^{\text {tail }}+r h_{\alpha^{\prime} \beta^{\prime} \gamma^{\prime}}^{\text {tail }}\left(u^{\gamma^{\prime}}+\Omega^{c} e_{c}^{\gamma^{\prime}}\right)+\mathcal{O}\left(m r^{2} / \mathcal{R}^{3}\right)\right] .
$$

This is the desired expansion of the metric perturbation in powers of $r / \mathcal{R}$. Our next task will be to calculate the components of this tensor in the retarded coordinates $\left(u, r \Omega^{a}\right)$.

The first step of this computation is to decompose $h_{\alpha \beta}$ in the tetrad $\left(e_{0}^{\alpha}, e_{a}^{\alpha}\right)$ that is obtained by parallel transport of $\left(u^{\alpha^{\prime}}, e_{a}^{\alpha^{\prime}}\right)$ on the null geodesic that links $x$ to its corresponding retarded point $x^{\prime} \equiv z(u)$ on the world line. (The vectors are parallel transported in the background spacetime.) The projections are

$$
\begin{aligned}
& h_{00}\left(u, r, \Omega^{a}\right) \equiv h_{\alpha \beta} e_{0}^{\alpha} e_{0}^{\beta}=\frac{2 m}{r}+h_{00}^{\mathrm{tail}}(u)+r\left[h_{000}^{\mathrm{tail}}(u)+h_{00 c}^{\mathrm{tail}}(u) \Omega^{c}\right]+\mathcal{O}\left(m r^{2} / \mathcal{R}^{3}\right), \\
& h_{0 b}\left(u, r, \Omega^{a}\right) \equiv h_{\alpha \beta} e_{0}^{\alpha} e_{b}^{\beta}=h_{0 b}^{\mathrm{tail}}(u)+r\left[h_{0 b 0}^{\mathrm{tail}}(u)+h_{0 b c}^{\mathrm{tail}}(u) \Omega^{c}\right]+\mathcal{O}\left(m r^{2} / \mathcal{R}^{3}\right), \\
& h_{a b}\left(u, r, \Omega^{a}\right) \equiv h_{\alpha \beta} e_{a}^{\alpha} e_{b}^{\beta}=\frac{2 m}{r} \delta_{a b}+h_{a b}^{\mathrm{tail}}(u)+r\left[h_{a b 0}^{\mathrm{tail}}(u)+h_{a b c}^{\mathrm{tail}}(u) \Omega^{c}\right]+\mathcal{O}\left(m r^{2} / \mathcal{R}^{3}\right) ;
\end{aligned}
$$

on the right-hand side we have the frame components of $h_{\alpha^{\prime} \beta^{\prime}}^{\text {tail }}$ and $h_{\alpha^{\prime} \beta^{\prime} \gamma^{\prime}}^{\text {tail }}$ taken with respect to the tetrad $\left(u^{\alpha^{\prime}}, e_{a}^{\alpha^{\prime}}\right)$; these are functions of retarded time $u$ only.

The perturbation is now expressed as

$$
h_{\alpha \beta}=h_{00} e_{\alpha}^{0} e_{\beta}^{0}+h_{0 b}\left(e_{\alpha}^{0} e_{\beta}^{b}+e_{\alpha}^{b} e_{\beta}^{0}\right)+h_{a b} e_{\alpha}^{a} e_{\beta}^{b},
$$

and its components are obtained by involving Equations (169) and (170), which list the components of the tetrad vectors in the retarded coordinates; this is the second (and longest) step of the computation. Noting that $a_{a}$ and $\omega_{a b}$ can both be set equal to zero in these equations (because they would produce negligible terms of order $m^{2}$ in $h_{\alpha \beta}$ ), and that $S_{a b}, S_{a}$, and $S$ can all be expressed in terms of the tidal fields $\mathcal{E}^{*}, \mathcal{E}_{a}^{*}, \mathcal{E}_{a b}^{*}, \mathcal{B}_{a}^{*}$, and $\mathcal{B}_{a b}^{*}$ using Equations $(204,205,206)$, we arrive at

$$
\begin{aligned}
h_{u u}= & \frac{2 m}{r}+h_{00}^{\text {tail }}+r\left(2 m \mathcal{E}^{*}+h_{000}^{\text {tail }}+h_{00 a}^{\text {tail }} \Omega^{a}\right)+\mathcal{O}\left(m r^{2} / \mathcal{R}^{3}\right), \\
h_{u a}= & \frac{2 m}{r} \Omega_{a}+h_{0 a}^{\text {tail }}+\Omega_{a} h_{00}^{\text {tail }}+r\left[2 m \mathcal{E}^{*} \Omega_{a}+\frac{2 m}{3}\left(\mathcal{E}_{a}^{*}+\mathcal{B}_{a}^{*}\right)+h_{0 a 0}^{\text {tail }}+\Omega_{a} h_{000}^{\text {tail }}+h_{0 a b}^{\text {tail }} \Omega^{b}+\Omega_{a} h_{00 b}^{\text {tail }} \Omega^{b}\right] \\
& +\mathcal{O}\left(m r^{2} / \mathcal{R}^{3}\right), \\
h_{a b}= & \frac{2 m}{r}\left(\delta_{a b}+\Omega_{a} \Omega_{b}\right)+\Omega_{a} \Omega_{b} h_{00}^{\text {tail }}+\Omega_{a} h_{0 b}^{\text {tail }}+\Omega_{b} h_{0 a}^{\text {tail }}+h_{a b}^{\text {tail }} \\
& +r\left[-\frac{2 m}{3}\left(\mathcal{E}_{a b}^{*}+\Omega_{a} \mathcal{E}_{b}^{*}+\mathcal{E}_{a}^{*} \Omega_{b}+\mathcal{B}_{a b}^{*}+\Omega_{a} \mathcal{B}_{b}^{*}+\Omega_{b} \mathcal{B}_{a}^{*}\right)+\Omega_{a} \Omega_{b}\left(h_{000}^{\text {tail }}+h_{00 c}^{\text {tail }} \Omega^{c}\right)\right. \\
& \left.\quad+\Omega_{a}\left(h_{0 b 0}^{\text {tail }}+h_{0 b c}^{\text {tail }} \Omega^{c}\right)+\Omega_{b}\left(h_{0 a 0}^{\text {tail }}+h_{0 a c}^{\text {tail }} \Omega^{c}\right)+\left(h_{a b 0}^{\text {tail }}+h_{a b c}^{\text {tail }} \Omega^{c}\right)\right]+\mathcal{O}\left(m r^{2} / \mathcal{R}^{3}\right) .
\end{aligned}
$$

Living Reviews in Relativity http://www . livingreviews . org/lrr-2004-6 
These are the coordinate components of the metric perturbation $h_{\alpha \beta}$ in the retarded coordinates $\left(u, r \Omega^{a}\right)$, expressed in terms of frame components of the tail fields $h_{\alpha^{\prime} \beta^{\prime}}^{\text {tails }}$ and $h_{\alpha^{\prime} \beta^{\prime} \gamma^{\prime}}^{\text {tails }}$. The perturbation is expanded in powers of $r / \mathcal{R}$ and it also involves the tidal gravitational fields of the background spacetime.

The external-zone metric is obtained by adding $g_{\alpha \beta}$ as given by Equations $(580,581,582)$ to $h_{\alpha \beta}$ as given by Equations $(593,594,595)$. The final result is

$$
\begin{aligned}
\mathrm{g}_{u u}= & -1-r^{2} \mathcal{E}^{*}+\mathcal{O}\left(r^{3} / \mathcal{R}^{3}\right) \\
& +\frac{2 m}{r}+h_{00}^{\text {tail }}+r\left(2 m \mathcal{E}^{*}-2 a_{a} \Omega^{a}+h_{000}^{\text {tail }}+h_{00 a}^{\text {tail }} \Omega^{a}\right)+\mathcal{O}\left(m r^{2} / \mathcal{R}^{3}\right), \\
\mathrm{g}_{u a}= & -\Omega_{a}+\frac{2}{3} r^{2}\left(\mathcal{E}_{a}^{*}+\mathcal{B}_{a}^{*}\right)+\mathcal{O}\left(r^{3} / \mathcal{R}^{3}\right) \\
& +\frac{2 m}{r} \Omega_{a}+h_{0 a}^{\text {tail }}+\Omega_{a} h_{00}^{\text {tail }} \\
& +r\left[2 m \mathcal{E}^{*} \Omega_{a}+\frac{2 m}{3}\left(\mathcal{E}_{a}^{*}+\mathcal{B}_{a}^{*}\right)+\left(\delta_{a}^{b}-\Omega_{a} \Omega^{b}\right) a_{b}-\omega_{a b} \Omega^{b}+h_{0 a 0}^{\text {tail }}+\Omega_{a} h_{000}^{\text {tail }}+h_{0 a b}^{\text {tail }} \Omega^{b}+\Omega_{a} h_{00 b}^{\text {tail }} \Omega^{b}\right] \\
& +\mathcal{O}\left(m r^{2} / \mathcal{R}^{3}\right), \\
\mathrm{g}_{a b}= & \delta_{a b}-\Omega_{a} \Omega_{b}-\frac{1}{3} r^{2}\left(\mathcal{E}_{a b}^{*}+\mathcal{B}_{a b}^{*}\right)+\mathcal{O}\left(r^{3} / \mathcal{R}^{3}\right) \\
& +\frac{2 m}{r}\left(\delta_{a b}+\Omega_{a} \Omega_{b}\right)+\Omega_{a} \Omega_{b} h_{00}^{\text {tail }}+\Omega_{a} h_{0 b}^{\text {tail }}+\Omega_{b} h_{0 a}^{\text {tail }}+h_{a b}^{\text {tail }} \\
& +r\left[-\frac{2 m}{3}\left(\mathcal{E}_{a b}^{*}+\Omega_{a} \mathcal{E}_{b}^{*}+\mathcal{E}_{a}^{*} \Omega_{b}+\mathcal{B}_{a b}^{*}+\Omega_{a} \mathcal{B}_{b}^{*}+\Omega_{b} \mathcal{B}_{a}^{*}\right)+\Omega_{a} \Omega_{b}\left(h_{000}^{\text {tail }}+h_{00 c}^{\text {tail }} \Omega^{c}\right)\right. \\
& \left.+\Omega_{a}\left(h_{0 b 0}^{\text {tail }}+h_{0 b c}^{\text {tail }} \Omega^{c}\right)+\Omega_{b}\left(h_{0 a 0}^{\text {tail }}+h_{0 a c}^{\text {tail }} \Omega^{c}\right)+\left(h_{a b 0}^{\text {tail }}+h_{a b c}^{\text {tail }} \Omega^{c}\right)\right]+\mathcal{O}\left(m r^{2} / \mathcal{R}^{3}\right) .
\end{aligned}
$$

Because $h_{\alpha^{\prime} \beta^{\prime}}^{\text {tails }}$ is of order $m / \mathcal{R}$ and $h_{\alpha^{\prime} \beta^{\prime} \gamma^{\prime}}^{\text {tails }}$ of order $m / \mathcal{R}^{2}$, we see that the metric possesses the buffer-zone form $\mathrm{g}=\eta \oplus r^{2} / \mathcal{R}^{2} \oplus m / r \oplus m / \mathcal{R} \oplus m r / \mathcal{R}^{2}$ that was anticipated in Equation (558). Notice that the expansion adopted here does not contain a term at order $r / \mathcal{R}$ and presumes that $a_{a}$ and $\omega_{a b}$ are both of order $m / \mathcal{R}^{2}$; this will be confirmed in Section 5.4.5.

\subsubsection{Transformation from external to internal coordinates}

Comparison of Equations $(568,569,570)$ and Equations $(596,597,598)$ reveals that the internalzone and external-zone metrics do no match in the buffer zone. But as the metrics are expressed in two different coordinate systems, this mismatch is hardly surprising. A meaningful comparison of the two metrics must therefore come after a transformation from the external coordinates $\left(u, r \Omega^{a}\right)$ to the internal coordinates $\left(\bar{u}, \bar{r} \bar{\Omega}^{a}\right)$. Our task in this section is to construct this coordinate transformation. We shall proceed in three stages. The first stage of the transformation, $\left(u, r \Omega^{a}\right) \rightarrow\left(u^{\prime}, r^{\prime} \Omega^{\prime a}\right)$, will be seen to remove unwanted terms of order $m / r$ in $\mathrm{g}_{\alpha \beta}$. The second stage, $\left(u^{\prime}, r^{\prime} \Omega^{\prime a}\right) \rightarrow\left(u^{\prime \prime}, r^{\prime \prime} \Omega^{\prime \prime a}\right)$, will remove all terms of order $m / \mathcal{R}$ in $\mathrm{g}_{\alpha^{\prime} \beta^{\prime}}$. Finally, the third stage $\left(u^{\prime \prime}, r^{\prime \prime} \Omega^{\prime \prime a}\right) \rightarrow\left(\bar{u}, \bar{r} \bar{\Omega}^{a}\right)$ will produce the desired internal coordinates.

The first stage of the coordinate transformation is

$$
\begin{aligned}
u^{\prime} & =u-2 m \ln r, \\
x^{\prime a} & =\left(1+\frac{m}{r}\right) x^{a},
\end{aligned}
$$

and it affects the metric at orders $m / r$ and $m r / \mathcal{R}^{2}$. This transformation redefines the radial coordinate $-r \rightarrow r^{\prime}=r+m$ - and incorporates in $u^{\prime}$ the gravitational time delay contributed by 
the small mass $m$. After performing the coordinate transformation the metric becomes

$$
\begin{aligned}
& \mathrm{g}_{u^{\prime} u^{\prime}}=-1-r^{\prime 2} \mathcal{E}^{\prime *}+\mathcal{O}\left(r^{\prime 3} / \mathcal{R}^{3}\right) \\
& +\frac{2 m}{r^{\prime}}+h_{00}^{\text {tail }}+r^{\prime}\left(4 m \mathcal{E}^{\prime *}-2 a_{a} \Omega^{\prime a}+h_{000}^{\text {tail }}+h_{00 a}^{\text {tail }} \Omega^{\prime a}\right)+\mathcal{O}\left(m r^{\prime 2} / \mathcal{R}^{3}\right) \text {, } \\
& \mathrm{g}_{u^{\prime} a^{\prime}}=-\Omega_{a}^{\prime}+\frac{2}{3} r^{\prime 2}\left(\mathcal{E}_{a}^{*}+\mathcal{B}_{a}^{\prime *}\right)+\mathcal{O}\left(r^{\prime 3} / \mathcal{R}^{3}\right) \\
& +h_{0 a}^{\text {tail }}+\Omega_{a}^{\prime} h_{00}^{\text {tail }} \\
& +r^{\prime}\left[-\frac{4 m}{3}\left(\mathcal{E}_{a}^{\prime *}+\mathcal{B}_{a}^{\prime *}\right)+\left(\delta_{a}^{b}-\Omega_{a}^{\prime} \Omega^{\prime b}\right) a_{b}-\omega_{a b} \Omega^{\prime b}+h_{0 a 0}^{\text {tail }}+\Omega_{a}^{\prime} h_{000}^{\text {tail }}+h_{0 a b}^{\text {tail }} \Omega^{\prime b}+\Omega_{a}^{\prime} h_{00 b}^{\text {tail }} \Omega^{\prime b}\right] \\
& +\mathcal{O}\left(m r^{\prime 2} / \mathcal{R}^{3}\right), \\
& \mathrm{g}_{a^{\prime} b^{\prime}}=\delta_{a b}-\Omega_{a}^{\prime} \Omega_{b}^{\prime}-\frac{1}{3} r^{\prime 2}\left(\mathcal{E}_{a b}^{\prime *}+\mathcal{B}_{a b}^{* *}\right)+\mathcal{O}\left(r^{\prime 3} / \mathcal{R}^{3}\right) \\
& +\Omega_{a}^{\prime} \Omega_{b}^{\prime} h_{00}^{\text {tail }}+\Omega_{a}^{\prime} h_{0 b}^{\text {tail }}+\Omega_{b}^{\prime} h_{0 a}^{\text {tail }}+h_{a b}^{\text {tail }} \\
& +r^{\prime}\left[\frac{2 m}{3}\left(\mathcal{E}_{a b}^{\prime *}+\Omega_{a}^{\prime} \mathcal{E}_{b}^{\prime *}+\mathcal{E}_{a}^{\prime *} \Omega_{b}^{\prime}+\mathcal{B}_{a b}^{\prime *}+\Omega_{a}^{\prime} \mathcal{B}_{b}^{\prime *}+\Omega_{b}^{\prime} \mathcal{B}_{a}^{\prime *}\right)+\Omega_{a}^{\prime} \Omega_{b}^{\prime}\left(h_{000}^{\text {tail }}+h_{00 c}^{\text {tail }} \Omega^{\prime c}\right)\right. \\
& \left.+\Omega_{a}^{\prime}\left(h_{0 b 0}^{\mathrm{tail}}+h_{0 b c}^{\mathrm{tail}} \Omega^{\prime c}\right)+\Omega_{b}^{\prime}\left(h_{0 a 0}^{\mathrm{tail}}+h_{0 a c}^{\mathrm{tail}} \Omega^{\prime c}\right)+\left(h_{a b 0}^{\mathrm{tail}}+h_{a b c}^{\mathrm{tail}} \Omega^{\prime c}\right)\right] \\
& +\mathcal{O}\left(m r^{\prime 2} / \mathcal{R}^{3}\right) \text {. }
\end{aligned}
$$

This metric matches $\mathrm{g}$ (internal zone) at orders $1, r^{\prime 2} / \mathcal{R}^{2}$, and $m / r^{\prime}$, but there is still a mismatch at orders $m / \mathcal{R}$ and $m r^{\prime} / \mathcal{R}^{2}$.

The second stage of the coordinate transformation is

$$
\begin{aligned}
& u^{\prime \prime}=u^{\prime}-\frac{1}{2} \int^{u^{\prime}} h_{00}^{\text {tail }}\left(u^{\prime}\right) d u^{\prime}-\frac{1}{2} r^{\prime}\left[h_{00}^{\text {tail }}\left(u^{\prime}\right)+2 h_{0 a}^{\text {tail }}\left(u^{\prime}\right) \Omega^{\prime a}+h_{a b}^{\text {tail }}\left(u^{\prime}\right) \Omega^{\prime a} \Omega^{\prime b}\right], \\
& x_{a}^{\prime \prime}=x_{a}^{\prime}+\frac{1}{2} h_{a b}^{\text {tail }}\left(u^{\prime}\right) x^{\prime b},
\end{aligned}
$$

and it affects the metric at orders $m / \mathcal{R}$ and $m r / \mathcal{R}^{2}$. After performing this transformation the metric becomes

$$
\begin{aligned}
& \mathrm{g}_{u^{\prime \prime} u^{\prime \prime}}=-1-r^{\prime \prime 2} \mathcal{E}^{\prime \prime *}+\mathcal{O}\left(r^{\prime \prime 3} / \mathcal{R}^{3}\right) \\
& +\frac{2 m}{r^{\prime \prime}}+r^{\prime \prime}\left[4 m \mathcal{E}^{\prime \prime *}-2\left(a_{a}-\frac{1}{2} h_{00 a}^{\text {tail }}+h_{0 a 0}^{\text {tail }}\right) \Omega^{\prime \prime a}\right]+\mathcal{O}\left(m r^{\prime \prime 2} / \mathcal{R}^{3}\right), \\
& \mathrm{g}_{u^{\prime \prime} a^{\prime \prime}}=-\Omega_{a}^{\prime \prime}+\frac{2}{3} r^{\prime \prime 2}\left(\mathcal{E}_{a}^{\prime \prime *}+\mathcal{B}_{a}^{\prime \prime *}\right)+\mathcal{O}\left(r^{\prime \prime 3} / \mathcal{R}^{3}\right) \\
& +r^{\prime \prime}\left[-\frac{4 m}{3}\left(\mathcal{E}_{a}^{\prime \prime *}+\mathcal{B}_{a}^{\prime \prime *}\right)-2 m \mathcal{E}_{a b}{\Omega^{\prime \prime b}}^{\prime b}\left(\delta_{a}^{b}-\Omega_{a}^{\prime \prime} \Omega^{\prime \prime b}\right)\left(a_{b}-\frac{1}{2} h_{00 b}^{\text {tail }}+h_{0 b 0}^{\text {tail }}\right)-\omega_{a b} \Omega^{\prime \prime b}\right. \\
& \left.+\frac{1}{2} \Omega_{a}^{\prime \prime} h_{000}^{\text {tail }}-\frac{1}{2} h_{a b 0}^{\text {tail }} \Omega^{\prime \prime b}+h_{0 a b}^{\text {tail }} \Omega^{\prime \prime b}+\frac{1}{2}\left(\delta_{a}^{b}+\Omega_{a}^{\prime \prime} \Omega^{\prime \prime b}\right) h_{00 b}^{\text {tail }}\right]+\mathcal{O}\left(m r^{\prime \prime 2} / \mathcal{R}^{3}\right), \\
& \mathrm{g}_{a^{\prime \prime} b^{\prime \prime}}=\delta_{a b}-\Omega_{a}^{\prime \prime} \Omega_{b}^{\prime \prime}-\frac{1}{3} r^{\prime \prime 2}\left(\mathcal{E}_{a b}^{\prime \prime *}+\mathcal{B}_{a b}^{\prime \prime *}\right)+\mathcal{O}\left(r^{\prime \prime 3} / \mathcal{R}^{3}\right) \\
& +r^{\prime \prime}\left[\frac{2 m}{3}\left(\mathcal{E}_{a b}^{\prime \prime *}+\Omega_{a}^{\prime \prime} \mathcal{E}_{b}^{\prime \prime *}+\mathcal{E}_{a}^{\prime \prime *} \Omega_{b}^{\prime \prime}+\mathcal{B}_{a b}^{\prime \prime *}+\Omega_{a}^{\prime \prime} \mathcal{B}_{b}^{\prime \prime *}+\Omega_{b}^{\prime \prime} \mathcal{B}_{a}^{\prime \prime *}\right)+\Omega_{a}^{\prime \prime} \Omega_{b}^{\prime \prime}\left(h_{000}^{\text {tail }}+h_{00 c}^{\text {tail }} \Omega^{\prime \prime c}\right)\right. \\
& \left.+\Omega_{a}^{\prime \prime}\left(h_{0 b 0}^{\text {tail }}+h_{0 b c}^{\text {tail }} \Omega^{\prime \prime c}\right)+\Omega_{b}^{\prime \prime}\left(h_{0 a 0}^{\text {tail }}+h_{0 a c}^{\text {tail }} \Omega^{\prime \prime c}\right)+\left(h_{a b 0}^{\text {tail }}+h_{a b c}^{\text {tail }} \Omega^{\prime \prime c}\right)\right] \\
& +\mathcal{O}\left(m r^{\prime \prime 2} / \mathcal{R}^{3}\right)
\end{aligned}
$$


To arrive at these expressions we had to involve the relations

$$
\frac{d}{d u^{\prime \prime}} h_{00}^{\text {tail }}=h_{000}^{\text {tail }}, \quad \frac{d}{d u^{\prime \prime}} h_{0 a}^{\text {tail }}=h_{0 a 0}^{\text {tail }}, \quad \frac{d}{d u^{\prime \prime}} h_{a b}^{\text {tail }}=4 m \mathcal{E}_{a b}+h_{a b 0}^{\text {tail }},
$$

which are obtained by covariant differentiation of Equation (587) with respect to $u$. The metric now matches $\mathrm{g}$ (internal zone) at orders $1, r^{\prime \prime 2} / \mathcal{R}^{2}, m / r^{\prime \prime}$, and $m / \mathcal{R}$, but there is still a mismatch at order $m r^{\prime \prime} / \mathcal{R}^{2}$.

The third and final stage of the coordinate transformation is

$$
\begin{aligned}
\bar{u}= & u^{\prime \prime}-\frac{1}{4} r^{\prime \prime 2}\left[h_{000}^{\mathrm{tail}}+\left(h_{00 a}^{\mathrm{tail}}+2 h_{0 a 0}^{\mathrm{tail}}\right) \Omega^{\prime \prime a}+\left(h_{a b 0}^{\mathrm{tail}}+2 h_{0 a b}^{\mathrm{tail}}\right) \Omega^{\prime \prime a}{\Omega^{\prime \prime b}}^{\prime b}+h_{a b c}^{\mathrm{tail}} \Omega^{\prime \prime a} \Omega^{\prime \prime b} \Omega^{\prime \prime c}\right], \\
\bar{x}_{a}= & \left(1+\frac{m}{3} r^{\prime \prime} \mathcal{E}_{b c} \Omega^{\prime \prime b} \Omega^{\prime \prime c}\right) x_{a}^{\prime \prime} \\
& +\frac{1}{2} r^{\prime \prime 2}\left[-\frac{1}{2} h_{00 a}^{\mathrm{tail}}+h_{0 a 0}^{\mathrm{tail}}+\left(h_{0 a b}^{\mathrm{tail}}-h_{0 b a}^{\mathrm{tail}}+h_{a b 0}^{\mathrm{tail}}+\frac{4 m}{3} \mathcal{E}_{a b}\right) \Omega^{\prime \prime b}+\left(Q_{a b c}-Q_{b c a}+Q_{c a b}\right) \Omega^{\prime \prime b} \Omega^{\prime \prime c}\right],
\end{aligned}
$$

where

$$
Q_{a b c}=\frac{1}{2} h_{a b c}^{\mathrm{tail}}+\frac{m}{3}\left(\varepsilon_{a c d} \mathcal{B}_{b}^{d}+\varepsilon_{b c d} \mathcal{B}_{a}^{d}\right) .
$$

This transformation puts the metric in its final form

$$
\begin{aligned}
\mathrm{g}_{\bar{u} \bar{u}}= & -1-\bar{r}^{2} \overline{\mathcal{E}}^{*}+\mathcal{O}\left(\bar{r}^{3} / \mathcal{R}^{3}\right) \\
& +\frac{2 m}{\bar{r}}+\bar{r}\left[4 m \overline{\mathcal{E}}^{*}-2\left(a_{a}-\frac{1}{2} h_{00 a}^{\text {tail }}+h_{0 a 0}^{\text {tail }}\right) \bar{\Omega}^{a}\right]+\mathcal{O}\left(m \bar{r}^{2} / \mathcal{R}^{3}\right) \\
\mathrm{g}_{\bar{u} \bar{a}}= & -\bar{\Omega}_{a}+\frac{2}{3} \bar{r}^{2}\left(\overline{\mathcal{E}}_{a}^{*}+\overline{\mathcal{B}}_{a}^{*}\right)+\mathcal{O}\left(\bar{r}^{3} / \mathcal{R}^{3}\right) \\
& +\bar{r}\left[-\frac{4 m}{3}\left(\overline{\mathcal{E}}_{a}^{*}+\overline{\mathcal{B}}_{a}^{*}\right)+\left(\delta_{a}^{b}-\bar{\Omega}_{a} \bar{\Omega}^{b}\right)\left(a_{b}-\frac{1}{2} h_{00 b}^{\text {tail }}+h_{0 b 0}^{\text {tail }}\right)-\left(\omega_{a b}-h_{0[a b]}^{\text {tail }}\right) \bar{\Omega}^{b}\right] \\
& +\mathcal{O}\left(m \bar{r}^{2} / \mathcal{R}^{3}\right) \\
\mathrm{g}_{\bar{a} \bar{b}}= & \delta_{a b}-\bar{\Omega}_{a} \bar{\Omega}_{b}-\frac{1}{3} \bar{r}^{2}\left(\overline{\mathcal{E}}_{a b}^{*}+\overline{\mathcal{B}}_{a b}^{*}\right)+\mathcal{O}\left(\bar{r}^{3} / \mathcal{R}^{3}\right)+\mathcal{O}\left(m \bar{r}^{2} / \mathcal{R}^{3}\right) .
\end{aligned}
$$

Except for the terms involving $a_{a}$ and $\omega_{a b}$, this metric is equal to $\mathrm{g}$ (internal zone) as given by Equations $(568,569,570)$ linearized with respect to $m$.

\subsubsection{Motion of the black hole in the background spacetime}

A precise match between $\mathrm{g}$ (external zone) and $\mathrm{g}$ (internal zone) is produced when we impose the relations

$$
a_{a}=\frac{1}{2} h_{00 a}^{\mathrm{tail}}-h_{0 a 0}^{\mathrm{tail}}
$$

and

$$
\omega_{a b}=h_{0[a b]}^{\mathrm{tail}} .
$$

While Equation (616) tells us how the black hole moves in the background spacetime, Equation (617) indicates that the vectors $e_{a}^{\mu}$ are not Fermi-Walker transported on the world line.

The black hole's acceleration vector $a^{\mu}=a^{a} e_{a}^{\mu}$ can be constructed from the frame components listed in Equation (616). A straightforward computation gives

$$
a^{\mu}=-\frac{1}{2}\left(g^{\mu \nu}+u^{\mu} u^{\nu}\right)\left(2 h_{\nu \lambda \rho}^{\text {tail }}-h_{\lambda \rho \nu}^{\text {tail }}\right) u^{\lambda} u^{\rho},
$$


where the tail integral

$$
h_{\mu \nu \lambda}^{\text {tail }}=4 m \int_{-\infty}^{\tau^{-}} \nabla_{\lambda}\left(G_{+\mu \nu \mu^{\prime} \nu^{\prime}}-\frac{1}{2} g_{\mu \nu} G_{+\rho \mu^{\prime} \nu^{\prime}}^{\rho}\right)\left(z(\tau), z\left(\tau^{\prime}\right)\right) u^{\mu^{\prime}} u^{\nu^{\prime}} d \tau^{\prime}
$$

was previously defined by Equation (588). These are the MiSaTaQuWa equations of motion, exactly as they were written down in Equation (550). While the initial derivation of this result was based upon formal manipulations of singular quantities, the present derivation involves only well-behaved fields and is free of any questionable aspect. Such a derivation, based on matched asymptotic expansions, was first provided by Yasushi Mino, Misao Sasaki, and Takahiro Tanaka in 1997 [39].

Substituting Equations (616) and (617) into Equation (579) gives the following transport equation for the tetrad vectors:

$$
\frac{D e_{a}^{\mu}}{d \tau}=-\frac{1}{2} u^{\mu}\left(2 h_{\nu \lambda \rho}^{\mathrm{tail}}-h_{\nu \rho \lambda}^{\mathrm{tail}}\right) u^{\nu} e_{a}^{\lambda} u^{\rho}+\left(g^{\mu \rho}+u^{\mu} u^{\rho}\right) h_{\nu[\lambda \rho]}^{\mathrm{tail}} u^{\nu} e_{a}^{\lambda} .
$$

This can also be written in the alternative form

$$
\frac{D e_{a}^{\mu}}{d \tau}=-\frac{1}{2}\left(u^{\mu} e_{a}^{\lambda} u^{\rho}+g^{\mu \lambda} e_{a}^{\rho}-g^{\mu \rho} e_{a}^{\lambda}\right) u^{\nu} h_{\nu \lambda \rho}^{\text {tail }}
$$

that was first proposed by Mino, Sasaki, and Tanaka. Both equations state that in the background spacetime, the tetrad vectors are not Fermi-Walker transported on $\gamma$; the rotation tensor is nonzero and given by Equation (617).

\subsection{Concluding remarks}

I have presented a number of derivations of the equations that determine the motion of a point scalar charge $q$, a point electric charge $e$, and a point mass $m$ in a specified background spacetime. In this concluding section I summarize these derivations, and identify their strengths and weaknesses. I also describe the challenges that lie ahead in the concrete evaluation of the self-forces, most especially in the gravitational case.

\subsubsection{Conservation of energy-momentum}

For each of the three cases (scalar, electromagnetic, and gravitational) I have presented two different derivations of the equations of motion. The first derivation is based on a spatial averaging of the retarded field, and the second is based on a decomposition of the retarded field into singular and radiative fields. In the gravitational case, a third derivation, based on matched asymptotic expansions, was also presented. These derivations will be reviewed below, but I want first to explain why I have omitted to present a fourth derivation, based on energy-momentum conservation, in spite of the fact that historically, it is one of the most important.

Conservation of energy-momentum was used by Dirac [25] to derive the equations of motion of a point electric charge in flat spacetime, and the same method was adopted by DeWitt and Brehme [24] in their generalization of Dirac's work to curved spacetimes. This method was also one of the starting points of Mino, Sasaki, and Tanaka [39] in their calculation of the gravitational self-force. I have not discussed this method for two reasons. First, it is technically more difficult to implement than the methods presented in this review (considerably longer computations are involved). Second, it is difficult to endow this method with an adequate level of rigour, to the point that it is perhaps less convincing than the methods presented in this review. While the level of rigour achieved in flat spacetime is now quite satisfactory [56], I do not believe the same can

Living Reviews in Relativity

http: //www . livingreviews . org/lrr-2004-6 
be said of the generalization to curved spacetimes. (But it should be possible to improve on this matter.)

The method is based on the conservation equation $T^{\alpha \beta}{ }_{\beta}=0$, where the stress-energy tensor $T^{\alpha \beta}$ includes a contribution from the particle and a contribution from the field; the particle's contribution is a Dirac functional on the world line, and the field's contribution diverges as $1 / r^{4}$ near the world line. (I am using retarded coordinates in this discussion.) While in flat spacetime the differential statement of energy-momentum conservation can immediately be turned into an integral statement, the same is not true in a curved spacetime (unless the spacetime possesses at least one Killing vector). To proceed it is necessary to rewrite the conservation equation as

$$
0=g_{\alpha}^{\mu} T_{; \beta}^{\alpha \beta}=\left(g_{\alpha}^{\mu} T_{; \beta}^{\alpha \beta}\right)_{; \beta}-g_{\alpha ; \beta}^{\mu} T^{\alpha \beta},
$$

where $g_{\alpha}^{\mu}(z, x)$ is a parallel propagator from $x$ to an arbitrary point $z$ on the world line. Integrating this equation over the interior of a world-tube segment that consists of a "wall" of constant $r$ and two "caps" of constant $u$, we obtain

$$
0=\int_{\text {wall }} g_{\alpha}^{\mu} T^{\alpha \beta} d \Sigma_{\beta}+\int_{\text {caps }} g_{\alpha}^{\mu} T^{\alpha \beta} d \Sigma_{\beta}+\int_{\text {interior }} g_{\alpha ; \beta}^{\mu} T^{\alpha \beta} d V,
$$

where $d \Sigma_{\beta}$ is a three-dimensional surface element and $d V$ an invariant, four-dimensional volume element.

There is no obstacle in evaluating the wall integral, for which $T^{\alpha \beta}$ reduces to the field's stressenergy tensor; for a wall of radius $r$ the integral scales as $1 / r^{2}$. The integrations over the caps, however, are problematic: While the particle's contribution to the stress-energy tensor is integrable, the integration over the field's contribution goes as $\int_{0}^{r}\left(r^{\prime}\right)^{-2} d r^{\prime}$ and diverges. To properly regularize this integral requires great care, and the removal of all singular terms can be achieved by mass renormalization [24]. This issue arises also in flat spacetime [25], and while it is plausible that the rigourous distributional methods presented in [56] could be generalized to curved spacetimes, this remains to be done. More troublesome, however, is the interior integral, which does not appear in flat spacetime. Because $g_{\alpha ; \beta}^{\mu}$ scales as $r$, this integral goes as $\int_{0}^{r}\left(r^{\prime}\right)^{-1} d r^{\prime}$ and it also diverges, albeit less strongly than the caps integration. While simply discarding this integral produces the correct equations of motion, it would be desirable to go through a careful regularization of the interior integration, and provide a convincing reason to discard it altogether. To the best of my knowledge, this has not been done.

\subsubsection{Averaging method}

To identify the strengths and weaknesses of the averaging method it is convenient to adopt the Detweiler-Whiting decomposition of the retarded field into singular and radiative pieces. For concreteness I shall focus my attention on the electromagnetic case, and write

$$
F_{\alpha \beta}=F_{\alpha \beta}^{\mathrm{S}}+F_{\alpha \beta}^{\mathrm{R}} .
$$

Recall that this decomposition is unambiguous, and that the retarded and singular fields share the same singularity structure near the world line. Recall also that the retarded and singular fields satisfy the same field equations (with a distributional current density on the right-hand side), but that the radiative field is sourcefree.

To formulate equations of motion for the point charge we temporarily model it as a spherical hollow shell, and we obtain the net force acting on this object by averaging $F_{\alpha \beta}$ over the shell's surface. (The averaging is performed in the shell's rest frame, and the shell is spherical in the sense that its proper distance from the world line is the same in all directions.) The averaged field is 
next evaluated on the world line, in the limit of a zero-radius shell. Because the radiative field is smooth on the world line, this yields

$$
e\left\langle F_{\mu \nu}\right\rangle u^{\nu}=e\left\langle F_{\mu \nu}^{\mathrm{S}}\right\rangle u^{\nu}+e F_{\mu \nu}^{\mathrm{R}} u^{\nu}
$$

where

$$
e\left\langle F_{\mu \nu}^{\mathrm{S}}\right\rangle u^{\nu}=-(\delta m) a_{\mu}, \quad \delta m=\lim _{s \rightarrow 0}\left(\frac{2}{3} \frac{e^{2}}{s}\right)
$$

and

$$
e F_{\mu \nu}^{\mathrm{R}} u^{\nu}=e^{2}\left(g_{\mu \nu}+u_{\mu} u_{\nu}\right)\left(\frac{2}{3} \dot{a}^{\nu}+\frac{1}{3} R_{\lambda}^{\nu} u^{\lambda}\right)+2 e^{2} u^{\nu} \int_{-\infty}^{\tau^{-}} \nabla_{[\mu} G_{\nu] \lambda^{\prime}}^{+}\left(z(\tau), z\left(\tau^{\prime}\right)\right) u^{\lambda^{\prime}} d \tau^{\prime}
$$

The equations of motion are then postulated to be $m a_{\mu}=e\left\langle F_{\mu \nu}\right\rangle u^{\nu}$, where $m$ is the particle's bare mass. With the preceding results we arrive at $m_{\mathrm{obs}} a_{\mu}=e F_{\mu \nu}^{\mathrm{R}} u^{\nu}$, where $m_{\mathrm{obs}} \equiv m+\delta m$ is the particle's observed (renormalized) inertial mass.

The averaging method is sound, but it is not immune to criticism. A first source of criticism concerns the specifics of the averaging procedure, in particular, the choice of a spherical surface over any other conceivable shape. Another source is a slight inconsistency of the method that gives rise to the famous " $4 / 3$ problem" [52]: The mass shift $\delta m$ is related to the shell's electrostatic energy $E=e^{2} /(2 s)$ by $\delta m=\frac{4}{3} E$ instead of the expected $\delta m=E$. This problem is likely due [45] to the fact that the field that is averaged over the surface of the shell is sourced by a point particle and not by the shell itself. It is plausible that a more careful treatment of the near-source field will eliminate both sources of criticism: We can expect that the field produced by an extended spherical object will give rise to a mass shift that equals the object's electrostatic energy, and the object's spherical shape would then fully justify a spherical averaging. (Considering other shapes might also be possible, but one would prefer to keep the object's structure simple and avoid introducing additional multipole moments.) Further work is required to clean up these details.

The averaging method is at the core of the approach followed by Quinn and Wald [49], who also average the retarded field over a spherical surface surrounding the particle. Their approach, however, also incorporates a "comparison axiom" that allows them to avoid renormalizing the mass.

\subsubsection{Detweiler-Whiting axiom}

The Detweiler-Whiting decomposition of the retarded field becomes most powerful when it is combined with the Detweiler-Whiting axiom, which asserts that

the singular field exerts no force on the particle (it merely contributes to the particle's inertia); the entire self-force arises from the action of the radiative field.

This axiom, which is motivated by the symmetric nature of the singular field, and also its causal structure, gives rise to the equations of motion $m a_{\mu}=e F_{\mu \nu}^{\mathrm{R}} u^{\nu}$, in agreement with the averaging method (but with an implicit, instead of explicit, mass shift). In this picture, the particle simply interacts with a free radiative field (whose origin can be traced to the particle's past), and the procedure of mass renormalization is sidestepped. In the scalar and electromagnetic cases, the picture of a particle interacting with a radiative field removes any tension between the nongeodesic motion of the charge and the principle of equivalence. In the gravitational case the DetweilerWhiting axiom produces the statement that the point mass $m$ moves on a geodesic in a spacetime whose metric $g_{\alpha \beta}+h_{\alpha \beta}^{\mathrm{R}}$ is nonsingular and a solution to the vacuum field equations. This is a conceptually powerful, and elegant, formulation of the MiSaTaQuWa equations of motion.

Living Reviews in Relativity

http://www. livingreviews.org/lrr-2004-6 


\subsubsection{Matched asymptotic expansions}

It is well known that in general relativity the motion of gravitating bodies is determined, along with the spacetime metric, by the Einstein field equations; the equations of motion are not separately imposed. This observation provides a means of deriving the MiSaTaQuWa equations without having to rely on the fiction of a point mass. In the method of matched asymptotic expansions, the small body is taken to be a nonrotating black hole, and its metric perturbed by the tidal gravitational field of the external universe is matched to the metric of the external universe perturbed by the black hole. The equations of motion are then recovered by demanding that the metric be a valid solution to the vacuum field equations. This method, which was the second starting point of Mino, Sasaki, and Tanaka [39], gives what is by far the most compelling derivation of the MiSaTaQuWa equations. Indeed, the method is entirely free of conceptual and technical pitfalls there are no singularities (except deep inside the black hole) and only retarded fields are employed.

The introduction of a point mass in a nonlinear theory of gravitation would appear at first sight to be severely misguided. The lesson learned here is that one can in fact get away with it. The derivation of the MiSaTaQuWa equations of motion based on the method of matched asymptotic expansions does indeed show that results obtained on the basis of a point-particle description can be reliable, in spite of all their questionable aspects. This is a remarkable observation, and one that carries a lot of convenience: It is much easier to implement the point-mass description than to perform the matching of two metrics in two coordinate systems.

\subsubsection{Evaluation of the gravitational self-force}

The concrete evaluation of the scalar, electromagnetic, and gravitational self-forces is made challenging by the need to first obtain the relevant retarded Green's function. Successes achieved in the past were reviewed in Section 1.10, and here I want to describe the challenges that lie ahead. I will focus on the specific task of computing the gravitational self-force acting on a point mass that moves in a background Kerr spacetime. This case is especially important because the motion of a small compact object around a massive (galactic) black hole is a promising source of low-frequency gravitational waves for the Laser Interferometer Space Antenna (LISA) [32]; to calculate these waves requires an accurate description of the motion, beyond the test-mass approximation which ignores the object's radiation reaction.

The gravitational self-acceleration is given by the MiSaTaQuWa expression, which I write in the form

$$
\frac{D u^{\mu}}{d \tau}=a^{\mu}\left[h^{\mathrm{R}}\right] \equiv-\frac{1}{2}\left(g^{\mu \nu}+u^{\mu} u^{\nu}\right)\left(2 h_{\nu \lambda ; \rho}^{\mathrm{R}}-h_{\lambda \rho ; \nu}^{\mathrm{R}}\right) u^{\lambda} u^{\rho},
$$

where $h_{\alpha \beta}^{\mathrm{R}}$ is the radiative part of the metric perturbation. Recall that this equation is equivalent to the statement that the small body moves on a geodesic of a spacetime with metric $g_{\alpha \beta}+h_{\alpha \beta}^{\mathrm{R}}$. Here $g_{\alpha \beta}$ is the Kerr metric, and we wish to calculate $a^{\mu}\left[h^{\mathrm{R}}\right]$ for a body moving in the Kerr spacetime. This calculation is challenging and it involves a large number of steps.

The first sequence of steps is concerned with the computation of the (retarded) metric perturbation $h_{\alpha \beta}$ produced by a point particle moving on a specified geodesic of the Kerr spacetime. A method for doing this was elaborated by Lousto and Whiting [34] and Ori [44], building on the pioneering work of Teukolsky [57], Chrzanowski [18], and Wald [61]. The procedure consists of

- solving the Teukolsky equation for one of the Newman-Penrose quantities $\psi_{0}$ and $\psi_{4}$ (which are complex components of the Weyl tensor) produced by the point particle;

- obtaining from $\psi_{0}$ or $\psi_{4}$ a related (Hertz) potential $\Psi$ by integrating an ordinary differential equation; 
- applying to $\Psi$ a number of differential operators to obtain the metric perturbation in a radiation gauge that differs from the Lorenz gauge; and

- performing a gauge transformation from the radiation gauge to the Lorenz gauge.

It is well known that the Teukolsky equation separates when $\psi_{0}$ or $\psi_{4}$ is expressed as a multipole expansion, summing over modes with (spheroidal-harmonic) indices $l$ and $m$. In fact, the procedure outlined above relies heavily on this mode decomposition, and the metric perturbation returned at the end of the procedure is also expressed as a sum over modes $h_{\alpha \beta}^{l}$. (For each $l, m$ ranges from $-l$ to $l$, and summation of $m$ over this range is henceforth understood.) From these, mode contributions to the self-acceleration can be computed: $a^{\mu}\left[h_{l}\right]$ is obtained from our preceding expression for the self-acceleration by substituting $h_{\alpha \beta}^{l}$ in place of $h_{\alpha \beta}^{\mathrm{R}}$. These mode contributions do not diverge on the world line, but $a^{\mu}\left[h_{l}\right]$ is discontinuous at the radial position of the particle. The sum over modes, on the other hand, does not converge, because the "bare" acceleration (constructed from the retarded field $h_{\alpha \beta}$ ) is formally infinite.

The next sequence of steps is concerned with the regularization of each $a^{\mu}\left[h_{l}\right]$ by removing the contribution from $h_{\alpha \beta}^{\mathrm{S}}[6,7,9,11,38,21]$. The singular field can be constructed locally in a neighbourhood of the particle, and then decomposed into modes of multipole order $l$. This gives rise to modes $a^{\mu}\left[h_{l}^{\mathrm{S}}\right]$ for the singular part of the self-acceleration; these are also finite and discontinuous, and their sum over $l$ also diverges. But the true modes $a^{\mu}\left[h_{l}^{\mathrm{R}}\right]=a^{\mu}\left[h_{l}\right]-a^{\mu}\left[h_{l}^{\mathrm{S}}\right]$ of the self-acceleration are continuous at the radial position of the particle, and their sum does converge to the particle's acceleration. (It might be noted that obtaining a mode decomposition of the singular field involves providing an extension of $h_{\alpha \beta}^{\mathrm{S}}$ on a sphere of constant radial coordinate, and then integrating over the angular coordinates. The arbitrariness of the extension introduces ambiguities in each $a^{\mu}\left[h_{l}^{\mathrm{S}}\right]$, but the ambiguity disappears after summing over $l$.)

The self-acceleration is thus obtained by first computing $a^{\mu}\left[h_{l}\right]$ from the metric perturbation derived from $\psi_{0}$ or $\psi_{4}$, then computing the counterterms $a^{\mu}\left[h_{l}^{\mathrm{S}}\right]$ by mode-decomposing the singular field, and finally summing over all $a^{\mu}\left[h_{l}^{\mathrm{R}}\right]=a^{\mu}\left[h_{l}\right]-a^{\mu}\left[h_{l}^{\mathrm{S}}\right]$. This procedure is lengthy and involved, and thus far it has not been brought to completion, except for the special case of a particle falling radially toward a nonrotating black hole [5]. In this regard it should be noted that the replacement of the central Kerr black hole by a Schwarzschild black hole simplifies the task considerably. In particular, because there exists a practical and well-developed formalism to describe the metric perturbations of a Schwarzschild spacetime [51, 59, 63], there is no necessity to rely on the Teukolsky formalism and the complicated reconstruction of the metric variables.

The procedure described above is lengthy and involved, but it is also incomplete. The reason is that the metric perturbations $h_{\alpha \beta}^{l}$ that can be recovered from $\psi_{0}$ or $\psi_{4}$ do not by themselves sum up to the complete gravitational perturbation produced by the moving particle. Missing are the perturbations derived from the other Newman-Penrose quantities: $\psi_{1}, \psi_{2}$, and $\psi_{3}$. While $\psi_{1}$ and $\psi_{3}$ can always be set to zero by an appropriate choice of null tetrad, $\psi_{2}$ contains such important physical information as the shifts in mass and angular-momentum parameters produced by the particle [60]. Because the mode decompositions of $\psi_{0}$ and $\psi_{4}$ start at $l=2$, we might colloquially say that what is missing from the above procedure are the " $l=0$ and $l=1$ " modes of the metric perturbations. It is not currently known how the procedure can be completed so as to incorporate all modes of the metric perturbations. Specializing to a Schwarzschild spacetime eliminates this difficulty, and in this context the low multipole modes have been studied for the special case of circular orbits [43, 22].

In view of these many difficulties (and I choose to stay silent on others, for example, the issue of relating metric perturbations in different gauges when the gauge transformation is singular on the world line), it is perhaps not too surprising that such a small number of concrete calculations have been presented to date. But progress in dealing with these difficulties has been steady, and the situation should change dramatically in the next few years.

Living Reviews in Relativity

http://www . livingreviews . org//rr-2004-6 


\subsubsection{Beyond the self-force}

The successful computation of the gravitational self-force is not the end of the road. After the difficulties reviewed in the preceding Section 5.5.5 have all been removed and the motion of the small body is finally calculated to order $m$, it will still be necessary to obtain gauge-invariant information associated with the body's corrected motion. Because the MiSaTaQuWa equations of motion are not by themselves gauge-invariant, this step will necessitate going beyond the self-force.

To see how this might be done, imagine that the small body is a pulsar, and that it emits light pulses at regular proper-time intervals. The motion of the pulsar around the central black hole modulates the pulse frequencies as measured at infinity, and information about the body's corrected motion is encoded in the times-of-arrival of the pulses. Because these can be measured directly by a distant observer, they clearly constitute gauge-invariant information. But the timesof-arrival are determined not only by the pulsar's motion, but also by the propagation of radiation in the perturbed spacetime. This example shows that to obtain gauge-invariant information, one must properly combine the MiSaTaQuWa equations of motion with the metric perturbations.

In the context of the Laser Interferometer Space Antenna, the relevant observable is the instrument's response to a gravitational wave, which is determined by gauge-invariant waveforms, $h_{+}$and $h_{\times}$. To calculate these is the ultimate goal of this research programme, and the challenges that lie ahead go well beyond what I have described thus far. To obtain the waveforms it will be necessary to solve the Einstein field equations to second order in perturbation theory.

To understand this, consider first the formulation of the first-order problem. Schematically, one introduces a perturbation $h$ that satisfies a wave equation $\square h=T[z]$ in the background spacetime, where $T[z]$ is the stress-energy tensor of the moving body, which is a functional of the world line $z(\tau)$. In first-order perturbation theory, the stress-energy tensor must be conserved in the background spacetime, and $z(\tau)$ must describe a geodesic. It follows that in first-order perturbation theory, the waveforms constructed from the perturbation $h$ contain no information about the body's corrected motion.

The first-order perturbation, however, can be used to correct the motion, which is now described by the world line $z(\tau)+\delta z(\tau)$. In a naive implementation of the self-force, one would now resolve the wave equation with a corrected stress-energy tensor, $\square h=T[z+\delta z]$, and the new waveforms constructed from $h$ would then incorporate information about the corrected motion. This implementation is naive because this information would not be gauge-invariant. In fact, to be consistent one would have to include all second-order terms in the wave equation, not just the ones that come from the corrected motion. Schematically, the new wave equation would have the form of $\square h=(1+h) T[z+\delta z]+(\nabla h)^{2}$, and this is much more difficult to solve than the naive problem (if only because the source term is now much more singular than the distributional singularity contained in the stress-energy tensor). But provided one can find a way to make this second-order problem well posed, and provided one can solve it (or at least the relevant part of it), the waveforms constructed from the second-order perturbation $h$ will be gauge invariant. In this way, information about the body's corrected motion will have properly been incorporated into the gravitational waveforms.

The story is far from being over. 


\section{Acknowledgments}

My understanding of the work presented in this review was shaped by a series of annual meetings named after the movie director Frank Capra. The first of these meetings took place in 1998 and was held at Capra's ranch in Southern California; the ranch now belongs to Caltech, Capra's alma mater. Subsequent meetings were held in Dublin, Pasadena, Potsdam, State College PA, and Kyoto. At these meetings and elsewhere I have enjoyed many instructive conversations with Warren Anderson, Patrick Brady, Claude Barrabès, Leor Barack, Lior Burko, Manuella Campanelli, Steve Detweiler, Eanna Flanagan, Scott Hughes, Werner Israel, Carlos Lousto, Yasushi Mino, Hiroyuki Nakano, Amos Ori, Misao Sasaki, Takahiro Tanaka, Bill Unruh, Bob Wald, Alan Wiseman, and Bernard Whiting. This work was supported by the Natural Sciences and Engineering Research Council of Canada.

Living Reviews in Relativity

http: //www . livingreviews . org/lrr-2004-6 


\section{References}

[1] Alvi, K., "Approximate binary-black-hole metric", Phys. Rev. D, 61, 124013-1-124013-19, (2000). For a related online version see: K. Alvi, "An approximate binary-black-hole metric", (December, 1999), [Online Los Alamos Archive Preprint]: cited on 02 April 2004, http://www.arxiv.org/abs/gr-qc/9912113. 1.11, 5.4.1

[2] Barack, L., "Self-force on a scalar particle in spherically symmetric spacetime via mode-sum regularization: Radial trajectories", Phys. Rev. D, 62, 084027-1-084027-21, (2000). For a related online version see: L. Barack, "Self-force on a scalar particle in spherically symmetric spacetime via mode-sum regularization: Radial trajectories", (May, 2000), [Online Los Alamos Archive Preprint]: cited on 02 April 2004,

http://www.arxiv.org/abs/gr-qc/0005042. 1.10

[3] Barack, L., "Gravitational self-force by mode sum regularization", Phys. Rev. D, 64, 0840211-084021-16, (2001). For a related online version see: L. Barack, "Gravitational self-force by mode sum regularization", (May, 2001), [Online Los Alamos Archive Preprint]: cited on 02 April 2004,

http://www.arxiv.org/abs/gr-qc/0105040. 1.10

[4] Barack, L., and Burko, L., "Radiation-reaction force on a particle plunging into a black hole", Phys. Rev. D, 62, 084040-1-084040-5, (2000). For a related online version see: L. Barack, et al., "Radiation-reaction force on a particle plunging into a black hole", (July, 2000), [Online Los Alamos Archive Preprint]: cited on 02 April 2004,

http://www.arxiv.org/abs/gr-qc/0007033. 1.10

[5] Barack, L., and Lousto, C.O., "Computing the gravitational self-force on a compact object plunging into a Schwarzschild black hole", Phys. Rev. D, 66, 061502-1-061502-5, (2002). For a related online version see: L. Barack, et al., "Computing the gravitational self-force on a compact object plunging into a Schwarzschild black hole", (May, 2002), [Online Los Alamos Archive Preprint]: cited on 02 April 2004,

http://www.arxiv.org/abs/gr-qc/0205043. 1.10, 5.5.5

[6] Barack, L., Mino, Y., Nakano, H., Ori, A., and Sasaki, M., "Calculating the Gravitational Self-Force in Schwarzschild Spacetime", Phys. Rev. Lett., 88, 091101-1-091101-4, (2002). For a related online version see: L. Barack, et al., "Calculating the gravitational self-force in Schwarzschild spacetime", (November, 2001), [Online Los Alamos Archive Preprint]: cited on 02 April 2004,

http://www.arxiv.org/abs/gr-qc/0111001. 1.10, 5.5.5

[7] Barack, L., and Ori, A., "Mode sum regularization approach for the self-force in black hole spacetime", Phys. Rev. D, 61, 061502-1-061502-5, (2000). For a related online version see: L. Barack, et al., "Mode sum regularization approach for the self-force in black hole spacetime", (December, 1999), [Online Los Alamos Archive Preprint]: cited on 02 April 2004, http://www.arxiv.org/abs/gr-qc/9912010. 1.10, 5.5.5

[8] Barack, L., and Ori, A., "Gravitational self-force and gauge transformations", Phys. Rev. D, 64, 124003-1-124003-13, (2001). For a related online version see: L. Barack, et al., "Gravitational self force and gauge transformations", (July, 2001), [Online Los Alamos Archive Preprint]: cited on 02 April 2004,

http://www.arxiv.org/abs/gr-qc/0107056. 1.9, 5.3.7 
[9] Barack, L., and Ori, A., "Regularization parameters for the self-force in Schwarzschild spacetime: Scalar case", Phys. Rev. D, 66, 084022-1-084022-15, (2002). For a related online version see: L. Barack, et al., "Regularization parameters for the self force in Schwarzschild spacetime: I. scalar case", (April, 2002), [Online Los Alamos Archive Preprint]: cited on 02 April 2004, http://www.arxiv.org/abs/gr-qc/0204093. 1.10, 5.5.5

[10] Barack, L., and Ori, A., "Gravitational Self-Force on a Particle Orbiting a Kerr Black Hole", Phys. Rev. Lett., 90, 111101-1-111101-4, (2003). For a related online version see: L. Barack, et al., "Gravitational self-force on a particle orbiting a Kerr black hole", (December, 2002), [Online Los Alamos Archive Preprint]: cited on 02 April 2004, http://www.arxiv.org/abs/gr-qc/0212103. 1.10

[11] Barack, L., and Ori, A., "Regularization parameters for the self-force in Schwarzschild spacetime. II. Gravitational and electromagnetic cases", Phys. Rev. D, 67, 024029-1-024029-11, (2003). For a related online version see: L. Barack, et al., "Regularization parameters for the self-force in Schwarzschild spacetime: II. gravitational and electromagnetic cases", (September, 2002), [Online Los Alamos Archive Preprint]: cited on 02 April 2004, http://www .arxiv.org/abs/gr-qc/0209072. 1.10, 5.5.5

[12] Burko, L.M., "Self-force approach to synchrotron radiation", Am. J. Phys., 68, 456-468, (2000). For a related online version see: L.M. Burko, "Self-force approach to synchrotron radiation", (February, 1999), [Online Los Alamos Archive Preprint]: cited on 02 April 2004, http://www . arxiv.org/abs/gr-qc/9902079. 1.10

[13] Burko, L.M., "Self-Force on a Particle in Orbit around a Black Hole", Phys. Rev. Lett., 84, 4529-4532, (2000). For a related online version see: L.M. Burko, "Self force on particle in orbit around a black hole", (March, 2000), [Online Los Alamos Archive Preprint]: cited on 02 April 2004,

http://www . arxiv.org/abs/gr-qc/0003074. 1.10

[14] Burko, L.M., "Self-force on static charges in Schwarzschild spacetime", Class. Quantum Grav., 17, 227-250, (2000). For a related online version see: L.M. Burko, "Self-force on static charges in Schwarzschild spacetime", (November, 1999), [Online Los Alamos Archive Preprint]: cited on 02 April 2004,

http://www . arxiv.org/abs/gr-qc/9911042. 1.10

[15] Burko, L.M., Harte, A.I., and Poisson, E., "Mass loss by a scalar charge in an expanding universe", Phys. Rev. D, 65, 124006-1-124006-11, (2002). For a related online version see: L.M. Burko, et al., "Mass loss by a scalar charge in an expanding universe", (January, 2002), [Online Los Alamos Archive Preprint]: cited on 02 April 2004, http: //www.arxiv.org/abs/gr-qc/0201020. 1.10, 4.3.6

[16] Burko, L.M., and Liu, Y.T., "Self-force on a scalar charge in the spacetime of a stationary, axisymmetric black hole", Phys. Rev. D, 64, 024006-1-024006-21, (2001). For a related online version see: L.M. Burko, et al., "Self force on a scalar charge in the spacetime of a stationary, axisymmetric black hole", (March, 2001), [Online Los Alamos Archive Preprint]: cited on 02 April 2004,

http://www . arxiv.org/abs/gr-qc/0103008. 1.10

[17] Burko, L.M., Liu, Y.T., and Soen, Y., "Self-force on charges in the spacetime of spherical shells", Phys. Rev. D, 63, 024015-1-024015-18, (2001). For a related online version see: L.M. Burko, et al., "Self force on charges in the spacetime of spherical shells", (August, 2000), [Online Los Alamos Archive Preprint]: cited on 02 April 2004, http://www . arxiv.org/abs/gr-qc/0008065. 1.10

Living Reviews in Relativity

http: //www. livingreviews. org/lrr-2004-6 
[18] Chrzanowski, P.L., "Vector potential and metric perturbations of a rotating black hole", Phys. Rev. D, 11, 2042-2062, (1975). 5.5.5

[19] D'Eath, P.D., Black holes: Gravitational interactions, (Clarendon Press, Oxford, U.K., 1996). 1.11, 5.4.1

[20] Detweiler, S., "Radiation Reaction and the Self-Force for a Point Mass in General Relativity", Phys. Rev. Lett., 86, 1931-1934, (2001). For a related online version see: S. Detweiler, "Radiation reaction and the self-force for a point mass in general relativity", (November, 2000), [Online Los Alamos Archive Preprint]: cited on 02 April 2004,

http://www. arxiv.org/abs/gr-qc/0011039. 1.11, 5.4.1

[21] Detweiler, S., Messaritaki, E., and Whiting, B.F., "Self-force of a scalar field for circular orbits about a Schwarzschild black hole", Phys. Rev. D, 67, 104016-1-104016-18, (2003). For a related online version see: S. Detweiler, et al., "Self-force of a scalar field for circular orbits about a Schwarzschild black hole", (May, 2002), [Online Los Alamos Archive Preprint]: cited on 02 April 2004,

http://www. arxiv.org/abs/gr-qc/0205079. 1.10, 5.5.5

[22] Detweiler, S., and Poisson, E., "Low multipole contributions to the gravitational self-force", Phys. Rev. D, 69, (2004). For a related online version see: S. Detweiler, et al., "Low multipole contributions to the gravitational self-force", (December, 2003), [Online Los Alamos Archive Preprint]: cited on 02 April 2004,

http://www.arxiv.org/abs/gr-qc/0312010. In press. 5.5.5

[23] Detweiler, S., and Whiting, B.F., "Self-force via a Green's function decomposition", Phys. Rev. D, 67, 024025-1-024025-5, (2003). For a related online version see: S. Detweiler, et al., "Self-force via a Green's function decomposition", (February, 2002), [Online Los Alamos Archive Preprint]: cited on 02 April 2004,

http://www.arxiv.org/abs/gr-qc/0202086. 1.4, 1.4, 1.9, 1.11, 4.3.5, 5.3.6

[24] DeWitt, B.S., and Brehme, R.W., "Radiation Damping in a Gravitational Field", Ann. Phys. (N.Y.), 9, 220-259, (1960). 1.1, 1.7, 1.11, 5.2.6, 5.5.1

[25] Dirac, P.A.M., "Classical theory of radiating electrons", Proc. R. Soc. London, Ser. A, 167, 148, (1938). 1.1, 1.2, 5.1.6, 5.5.1

[26] Flanagan, E.E., and Wald, R.M., "Does back reaction enforce the averaged null energy condition in semiclassical gravity?", Phys. Rev. D, 54, 6233-6283, (1996). For a related online version see: E.E. Flanagan, et al., "Does backreaction enforce the averaged null energy condition in semiclassical gravity?", (February, 1996), [Online Los Alamos Archive Preprint]: cited on 02 April 2004,

http://www.arxiv.org/abs/gr-qc/9602052. 1, 5.1.6

[27] Friedlander, F.G., The wave equation on a curved spacetime, (Cambridge University Press, Cambridge, U.K., 1975). 1.11

[28] Hadamard, J., Lectures on Cauchy's problem in linear partial differential equations, (Yale University Press, New Haven, CT, U.S.A., 1923). 1.11

[29] Hobbs, J.M., "A Vierbein Formalism for Radiation Damping", Ann. Phys. (N.Y.), 47, 141165, (1968). 1.1, 1.7, 5.2.6

[30] Jackson, J.D., Classical Electrodynamics, Third Edition, (Wiley, New York, NY, U.S.A., 1999). $1.2,1.2,5.1 .6$ 
[31] Kates, R.E., "Motion of a small body through an external field in general relativity calculated by matched asymptotic expansions", Phys. Rev. D, 22, 1853-1870, (1980). 1.11, 5.4.1

[32] ESA and NASA, "LISA Home Page", (2004), [Online HTML Document]: cited on 02 April 2004 ,

http://lisa.jpl.nasa.gov. 5.5 .5

[33] Lousto, C.O., "Pragmatic Approach to Gravitational Radiation Reaction in Binary Black Holes", Phys. Rev. Lett., 84, 5251-5254, (2000). For a related online version see: C.O. Lousto, "Pragmatic approach to gravitational radiation reaction in binary black holes", (December, 1999), [Online Los Alamos Archive Preprint]: cited on 02 April 2004, http://www.arxiv.org/abs/gr-qc/9912017. 1.10

[34] Lousto, C.O., and Whiting, B.F., "Reconstruction of black hole metric perturbations from the Weyl curvature", Phys. Rev. D, 66, 024026-1-024026-7, (2002). For a related online version see: C.O. Lousto, et al., "Reconstruction of Black Hole Metric Perturbations from Weyl Curvature", (March, 2002), [Online Los Alamos Archive Preprint]: cited on 02 April 2004,

http://www.arxiv.org/abs/gr-qc/0203061. 1.10, 5.5.5

[35] Manasse, F.K., "Distortion in the metric of a small center of gravitational attraction due to its proximity to a very large mass", J. Math. Phys., 4, 746-761, (1963). 1.11, 5.4.1

[36] Manasse, F.K., and Misner, C.W., "Fermi normal coordinates and some basic concepts in differential geometry", J. Math. Phys., 4, 735-745, (1963). 1.11

[37] Mino, Y., "Perturbative approach to an orbital evolution around a supermassive black hole", Phys. Rev. D, 67, 084027-1-084027-17, (2003). For a related online version see: Y. Mino, "Perturbative Approach to an orbital evolution around a Supermassive black hole", (February, 2003), [Online Los Alamos Archive Preprint]: cited on 02 April 2004,

http://www . arxiv.org/abs/gr-qc/0302075. 1.10

[38] Mino, Y., Nakano, H., and Sasaki, M., "Covariant Self-Force Regularization of a Particle Orbiting a Schwarzschild Black Hole - Mode Decomposition Regularization", Prog. Theor. Phys., 108, 1039-1064, (2003). For a related online version see: Y. Mino, et al., "Covariant Self-force Regularization of a Particle Orbiting a Schwarzschild Black Hole - Mode Decomposition Regularization -", (November, 2001), [Online Los Alamos Archive Preprint]: cited on 02 April 2004,

http://www . arxiv .org/abs/gr-qc/0111074. 1.10, 5.5.5

[39] Mino, Y., Sasaki, M., and Tanaka, T., "Gravitational radiation reaction to a particle motion", Phys. Rev. D, 55, 3457-3476, (1997). For a related online version see: Y. Mino, et al., "Gravitational Radiation Reaction to a Particle Motion", (June, 1996), [Online Los Alamos Archive Preprint]: cited on 02 April 2004,

http: //www . arxiv.org/abs/gr-qc/9606018. 1.1, 1.9, 1.11, 5.3.6, 5.4.5, 5.5.1, 5.5.4

[40] Misner, C.W., Thorne, K.S., and Wheeler, J.A., Gravitation, (Freeman, San Francisco, CA, U.S.A., 1973). 1.11

[41] Morette-DeWitt, C., and DeWitt, B.S., "Falling charges", Physics (Long Island City, N.Y.), 1, 3, (1964). 1.10

[42] Morette-DeWitt, C., and Ging, J.L., "Freinage dû à la radiation gravitationnelle", C. R. Hebd. Seanc. Acad. Sci., 251, 1868, (1960). 5.3.6

Living Reviews in Relativity

http://www. livingreviews . org/lrr-2004-6 
[43] Nakano, H., Sago, N., and Sasaki, M., "Gauge problem in the gravitational self-force: First post Newtonian force under Regge-Wheeler gauge", Phys. Rev. D, 68, 124003-1-124003-31, (2003). For a related online version see: H. Nakano, et al., "Gauge Problem in the Gravitational Self-Force II. First Post Newtonian Force under Regge-Wheeler gauge", (August, 2003), [Online Los Alamos Archive Preprint]: cited on 02 April 2004,

http://www. arxiv.org/abs/gr-qc/0308027. 5.5 .5

[44] Ori, A., "Reconstruction of inhomogeneous metric perturbations and electromagnetic fourpotential in Kerr spacetime", Phys. Rev. D, 67, 124010-1-124010-19, (2003). For a related online version see: A. Ori, "Reconstruction of inhomogeneous metric perturbations and electromagnetic four-potential in Kerr spacetime", (July, 2002), [Online Los Alamos Archive Preprint]: cited on 02 April 2004,

http://www. arxiv.org/abs/gr-qc/0207045. 1.10, 5.5.5

[45] Ori, A., and Rosenthal, E., "Calculation of the self force using the extended-object approach", Phys. Rev. D, 68, 041701-1-041701-4, (2003). For a related online version see: A. Ori, et al., "Universal Self Force from an Extended-Object Approach", (May, 2002), [Online Los Alamos Archive Preprint]: cited on 02 April 2004,

http://www. arxiv.org/abs/gr-qc/0205003. $\quad 5.5 .2$

[46] Pfenning, M.J., and Poisson, E., "Scalar, electromagnetic, and gravitational self-forces in weakly curved spacetimes", Phys. Rev. D, 65, 084001-1-084001-30, (2002). For a related online version see: M.J. Pfenning, et al., "Scalar, electromagnetic, and gravitational self-forces in weakly curved spacetimes", (December, 2000), [Online Los Alamos Archive Preprint]: cited on 02 April 2004,

http://www.arxiv.org/abs/gr-qc/0012057. 1.10

[47] Poisson, E., "An introduction to the Lorentz-Dirac equation", (December, 1999), [Online Los Alamos Archive Preprint]: cited on 02 April 2004,

http://www.arxiv.org/abs/gr-qc/9912045. 1.2, 1, 5.1.6

[48] Quinn, T.C., "Axiomatic approach to radiation reaction of scalar point particles in curved spacetime", Phys. Rev. D, 62, 064029-1-064029-9, (2000). For a related online version see: T.C. Quinn, "An axiomatic approach to radiation reaction of scalar point particles in curved spacetime", (May, 2000), [Online Los Alamos Archive Preprint]: cited on 02 April 2004, http://www.arxiv.org/abs/gr-qc/0005030. 1.1, 1.8, 5.1.6

[49] Quinn, T.C., and Wald, R.M., "Axiomatic approach to electromagnetic and gravitational radiation reaction of particles in curved spacetime", Phys. Rev. D, 56, 3381-3394, (1997). For a related online version see: T.C. Quinn, et al., "Axiomatic approach to electromagnetic and gravitational radiation reaction of particles in curved spacetime", (October, 1996), [Online Los Alamos Archive Preprint]: cited on 02 April 2004,

http://www.arxiv.org/abs/gr-qc/9610053. 1.1, 1.9, 1.11, 5.2.6, 5.3.6, 5.5.2

[50] Quinn, T.C., and Wald, R.M., "Energy conservation for point particles undergoing radiation reaction", Phys. Rev. D, 60, 064009-1-064009-20, (1999). For a related online version see: T.C. Quinn, et al., "Energy conservation for point particles undergoing radiation reaction", (March, 1999), [Online Los Alamos Archive Preprint]: cited on 02 April 2004, http://www.arxiv.org/abs/gr-qc/9903014. 5.2.6, 5.3.6

[51] Regge, T., and Wheeler, J.A., "Stability of a Schwarzschild Singularity", Phys. Rev., 108, 1063-1069, (1957). 5.4.1, 5.5.5 
[52] Rohrlich, F., Classical charged particles, (Addison-Wesley, Redwood City, CA, U.S.A., 1990). $1.1,1.2,5.5 .2$

[53] Sciama, D.W., Waylen, P.C., and Gilman, R.C., "Generally Covariant Integral Formulation of Einstein's Field Equations", Phys. Rev., 187, 1762-1766, (1969). 4.5.1

[54] Smith, A.G., and Will, C.M., "Force on a static charge outside a Schwarzschild black hole", Phys. Rev. D, 22, 1276-1284, (1980). 1.10

[55] Synge, J.L., Relativity: The General Theory, (North-Holland, Amsterdam, The Netherlands, 1960). $\quad 1.5,1.11$

[56] Teitelboim, C., Villarroel, D., and van Weert, C.G., "Classical electrodynamics of retarded fields and point particles", Riv. Nuovo Cimento, 3, 9, (1980). 1.2, 5.5.1

[57] Teukolsky, S.A., "Perturbations of a rotating black hole. I. Fundamental equations for gravitational, electromagnetic, and neutrino-field perturbations", Astrophys. J., 185, 635-647, (1973). $\quad 5.5 .5$

[58] Thorne, K.S., and Hartle, J.B., "Laws of motion and precession for black holes and other bodies", Phys. Rev. D, 31, 1815-1837, (1985). 1.11, 3.2.6, 5.4.1, 5.4.2

[59] Vishveshwara, C.V., "Stability of the Schwarzschild Metric", Phys. Rev. D, 1, 2870-2879, (1970). $\quad 5.4 .1,5.5 .5$

[60] Wald, R.M., "On perturbations of a Kerr black hole", J. Math. Phys., 14, 1453-1461, (1973). 5.5 .5

[61] Wald, R.M., "Construction of Solutions of Gravitational, Electromagnetic, or Other Perturbation Equations from Solutions of Decoupled Equations", Phys. Rev. Lett., 41, 203-206, (1978). $\quad 5.5 .5$

[62] Wiseman, A.G., "Self-force on a static scalar test charge outside a Schwarzschild black hole", Phys. Rev. D, 61, 084014-1-084014-14, (2000). For a related online version see: A.G. Wiseman, "The self-force on a static scalar test-charge outside a Schwarzschild black hole", (January, 2000), [Online Los Alamos Archive Preprint]: cited on 02 April 2004, http://www.arxiv.org/abs/gr-qc/0001025. 1.10

[63] Zerilli, F.J., "Gravitational field of a particle falling in a Schwarzschild geometry analyzed in tensor harmonics", Phys. Rev. D, 2, 2141-2160, (1970). 5.4.1, 5.4.2, 5.5.5

[64] Zhang, X.-H., "Multipole expansions of the general-relativistic gravitational field of the external universe", Phys. Rev. D, 34, 991-1004, (1986). 3.2 .6

Living Reviews in Relativity

http://www. livingreviews.org/lrr-2004-6 